\title{
The 4th Asia-Pacific Primary Liver Cancer Expert Meeting
}

\section{A Bridge to a Consensus on HCC Management}

July 5-7, 2013, Busan, Korea

Guest Editors

Kwang-Hyub Han, Seoul

Masatoshi Kudo, Osaka

Sheng-Long Ye, Shanghai KARGER $\begin{aligned} & \text { Basel } \cdot \text { Freiburg } \cdot \text { Paris } \cdot \text { London } \cdot \text { New York } \cdot \text { New Delhi } \cdot \text { Bangkok } \\ & \text { Beijing } \cdot \text { Tokyo } \cdot \text { Kuala Lumpur } \cdot \text { Singapore } \cdot \text { Sydney }\end{aligned}$ 


\section{Curriculum Vitae}

Name

Position

Institution

E-mail
Kwang-Hyub Han

Severance Hospital, Yonsei University College of Medicine gihankhys@yuhs.ac
Professor

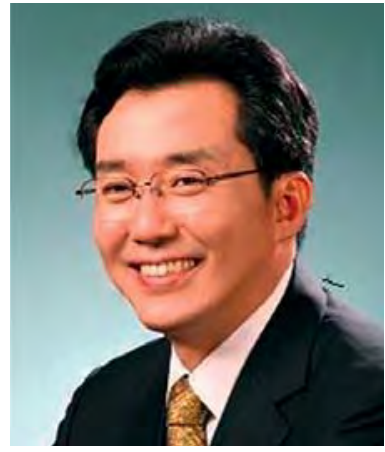

Kwang-Hyub Han, MD, is chairman of department of Internal Medicine, and staff of gastroenterologist (hepatologist). He is also a professor of internal medicine at the university's College of Medicine, from which he earned his medical degree (1979). He is the director and principal investigator of a 9-year project, “Clinical Research Center for Liver Cirrhosis," granted from the Ministry of Health and Welfare and Health Technology Planning and Evaluation Board. He organized the $1^{\text {st }}$ Asian-Pacific Primary Liver Cancer Expert (APPLE) meeting as a co-chairman. He was a governing board member of the International Liver Cancer Association and the president of Korea Liver Cancer Study. He served as a committee member of the working party for the Asian-Pacific consensus statement on hepatocellular carcinoma guidelines 2008 .

\section{Research Interest:}

Liver Cancer and Hepatism. 


\section{Health Burden of Viral Hepatitis in Asia: Past, Present \& Future}

\section{Kwang-Hyub Han}

Severance Hospital, Yonsei University College of Medicine, Seoul, Korea

- Viral hepatitis represents one of the major threats to global health, with chronic hepatitis B and C estimated to affect over 500 million people worldwide ${ }^{1}$.

- Chronic hepatitis B and C are devastating diseases and kill one million people every year ${ }^{2}$.

- 500,000-750,000 death attributable to hepatitis B

- 250,000 death attributable to in hepatitis C

- Asia Pacific shoulders a significant proportion of the global disease burden of hepatitis $\mathrm{B}^{1}$ and hepatitis $\mathrm{C}^{2}$.

- Approximately 340 million people living with chronic hepatitis B or C in Asia Pacific, which is $65 \%$ of the total number worldwide.

- Prevalance of hepatitis B virus (HBV) infection.

- Two billion peoples worldwide

- The $10^{\text {th }}$ leading cause of death worldwide

- 600,000 death each year from HBV related liver disease or hepatocellular carcinoma (HCC).

- In Asia-Pacific regions

- Chronic HBV infection is common

- One of three major causes of death in Asia and pacific regions.

- Health care costs during the first 7 month after diagnosis were 3.3 times higher in CHB patients ${ }^{1}$.

- Costs escalate with increasing severity of illness.

- Direct medical cost

- Indirect costs (e.g lost work days, productivity)

- The societal cost of hepatitis B in South Korea ${ }^{2}$

- The direct costs of the HBV disease is equivalent to $3.2 \%$ of the national health-care expenditure in 1997.

- Frequency

- Prevalence (pool of existing cases)

- Incidence (occurrence of new cases)

- Impact on

- Longevity (premature death)

- Morbidity (in- and outpatient care, quality of life)

- Finance (direct and indirect costs to society)

- 160 million people have been infected with hepatitis $\mathrm{C}$ virus (HCV) worldwide.

- China alone had more HCV infections than all of Europe or the america ${ }^{1}$.

- In Asia-Pacific regions

- Most countries had 1-2\% prevalance. However, some countries had higher prevalance.

- Pakistan 4.7\%, Taiwan 4.4\%, Mongolia >10\%

- Large geographical region has a great diversity of genotypes
- Genotypes 1 is common in China, Taiwan and north asia

- Genotypes 3 is dominant in India and Pakistan

- Genotypes 6 is common in Vietnam and southeast Asian

- In 1991, WHO Global Advisory Group of Expanded Program on Immunization (EPI) recommended national immunization programs in all countries with a hepatitis B carrier prevalence of $8 \%$ or greater.

- The estimated global coverage rate of infant HBV vaccination increased from less than $1 \%$ in 1990 to $30 \%$ in 2000 , and from nearly $50 \%$ in 2004 to $75 \%$ in 2010.

- As of 2010, 177 of the 193 WHO member states (92\%) had initiated HBV vaccination programs.

\section{Hepatitis B vaccination}

- In 1991, WHO Global Advisory Group of Expanded Program on Immunization (EPI) recommended national immunization programs in all countries with a hepatitis B carrier prevalence of $8 \%$ or greater.

- The estimated global coverage rate of infant HBV vaccination increased from less than $1 \%$ in 1990 to $30 \%$ in 2000 , and from nearly $50 \%$ in 2004 to $75 \%$ in 2010.

- As of 2010, 177 of the 193 WHO member states (92\%) had initiated HBV vaccination programs.

\section{Public Health Policy for Controlling Hcv}

- Implementation of high-quality surveillance system and reporting programs.

- Need of education programmes

- Common risk factor of HCV infection in Asiapacific area1.

- Nosocomial infection

- Blood transfusion

- Injection drug use

- Unique risk factor in some countries

- Public shaving beard and other hairs

- Tattooing

- Sexually transmitted HCV infection

To reduce the:

- Transmission of agents that cause viral hepatitis

- Morbidity and mortality due to viral hepatitis through improving the care of patients with viral hepatitis

- Socio-economic impact of viral hepatitis at individual, community and population levels.

\section{$\mathrm{HBV}$ in A-P region}

- Disease burden of HBV will be decreasing

- Although HBV incidence will be remarkably declined, the prevalence may be gradually declined

- HBV related HCC incidence will be proportionally decreasing

- Treatment efficacy in CHB will be remarkably improved, but the burden of antiviral therapy for HBV will be gradually increased in many A-P region.

- Optimal guideline for HBV concerning cost effectiveness will be necessary. 
$\mathrm{HCV}$ in $\mathrm{A}-\mathrm{P}$ region

- Burden of HCV will be increasing

- Although HCV incidence will be declined, the prevalence may remain high

- HCV related HCC incidence will be proportionally increasing

- Treatment efficacy in CHC will be remarkably improved, but effectiveness in community practice will not be remarkable

Future directions in Asia-Pacific region

- Building knowledge and capacity in the area of viral hepatitis

- Supporting the development of strong, integrated public health policies to address viral hepatitis

- Developing partnerships and collaborations with a range of individuals, organzations and governments committed to a reduction in the burden of viral hepatitis in Asia Pacific

- Advocacy - being an independent voice, connecting with other stakeholders, civil society and governments in calling for a better response to viral hepatitis

- Policy Development - conducting evidence-based policy and associated research to address data gaps and inform the development of public policy

- Education - building capacity at a local level by supporting local advocates with tools and knowledge to support their advocacy work

\section{Current issues and challenge in Asia}

At least two thirds of the people who die each year from HCC live in the Asia-Pacific region [3].

Mongolia is the country of the highest incidence and mortality rate in Asia

\section{Challenge: surveillance}

Screening and surveillance program are not implemented successfully in many Asian countries.

In the report from AP working party on prevention of HCC, the recommendation is that

Table Working party recommendations 11-14

Recommendations 11: Hepatocellular carcinoma (HCC) surveillance is a measure that can reduce mortality from HCC in subgroups of patients with cirrhosis, but is best conducted within an well-organized screening program (level III).

Recommendations 12: The recommended surveillance program outside of Japan is liver ultrasound conducted by an experienced centre every 6 months and serum $\alpha$-fetoprotein performed simultaneously (level III).

Recommendations 13: All patients with cirrhosis are recommended for HCC surveillance, unless comorbidity, logistics and patient choice render curative therapy inappropriate (level lia).

Recommendations 14: HCC screening is also recommended for high-risk patients with chronic hepatitis B (especially those aged $>30$ years with serum hepatitis B virus DNA levels $>20,000 \mathrm{IU} / \mathrm{mL}$ ) in the absence of a known diagnosis of cirrhosis (level III). ${ }^{39}$
At the liver cancer working group report, the surveillance program is basically similar among three countries but in cirrhotic patients, Japanese prefer to have shorter interval and more tumor markers

\section{Dx}

The majority of HCC patients in Asia still presents with intermediate and advanced stage HCC at diagnosis

Barriers for Surveillance and Diagnosis

- There is a lack of awareness for screening and surveillance among at-risk populations, health-care providers and policy-makers

- There is insufficient understanding of surveillance program, so inadequate public resources are being allocated to surveillance programs

- Limited resources of diagnostic imaging modality in many Asian countries make an accurate early diagnosis of HCC difficult.

* The majority of HCC patients in Asia still presents with intermediate and advanced stage HCC at diagnosis.

* In the first APPLE meeting, the significant differences in the treatment approach according to HCC stages were identified.

* A consensus for managing intermediate and advanced stage HCC cannot be easily drawn due to these disparities in clinical practices and guidelines among Asian countries.

Japan is the country where early HCC is commonly detected

* The majority of HCC patients in Asia still presents with intermediate and advanced stage HCC at diagnosis.

* In the first APPLE meeting, the significant differences in the treatment approach according to HCC stages were identified.

* A consensus for managing intermediate and advanced stage HCC cannot be easily drawn due to these disparities in clinical practices and guidelines among Asian countries.

* At present, inadequate public resources are being allocated to prevention, control and surveillance programs for HCC in developing Asian countries.

* The majority of Asian patients with HCCs are diagnosed at the advanced stages of presentation due to the lack of public awareness and nationwide surveillance program and limited resources of diagnostic modality. Therefore, potentially curative treatment for HCC is not feasible.

* To overcome barriers, education and training for health-care providers for HCC management might be necessary. 


\section{How to Control HCC in Asia Pacific Region! \\ Sunil Taneja ${ }^{1}$, S.K. Sarin ${ }^{2}$ \\ ${ }^{1}$ Assistant professor, Hepatology, ${ }^{2}$ Professor and Head, Department of Hepatology, New Delhi, India}

Hepatocellular carcinoma (HCC) is the fourth most frequently diagnosed tumor disease throughout the world and is one of the fastest growing cancers. Nearly $3 / 4$ of the patients with HCC are in the Asian region. In the vast majority of cases, those affected are high-risk patients with chronic viral hepatitis and/or liver cirrhosis, which means there is a clearly identifiable target group for HCC screening. However, early diagnosis is a reality only in a minority of patients and in the majority of cases the disease is already in an advanced stage at diagnosis. The mortality rates with HCC are very high and most patients present at an advanced stage. For reducing the mortality from HCC, preventive strategies and early detection is highly desirable.

Chronic viral infections with hepatitis B (HBV) and hepatitis $\mathrm{C}$ (HCV) have been the major causes of HCC till recently in countries like India, Bangladesh, Pakistan and Srilanaka. Lately the incidence of HCC due to non-alcoholic steatohepatitis and alcoholic liver disease has been found to be steadily increasing. Among patients with cirrhosis caused by hepatitis $B$ virus (HBV) and hepatitis $C$ virus (HCV), a 5 -year cumulative incidence of HCC ranges from 15-20\%. Of the more than 650 patients seen at our center in the past 4 years, we analyzed recently 302 patients; the etiology of cirrhosis was Hepatitis B in 93 (30.8\%), Hepatitis C in 56 (19\%), Cryptogenic in 50 (17\%), Alcohol in 47 (16\%), NASH in $36(12 \%)$ and others in $20(6 \%)$. Diabetes as a risk factor was present in 37 (12.3\%) of patients of HCC. The mean CTP and MELD score were 8.75 \pm 2.9 and $15.99 \pm 6.23$ respectively. The median AFP level was 203.5 (IQR 23.34-1801.5) and was much higher in patients with multifocal HCC 474.5 (IQR 116.7-15449). Tumor thrombus involving the branch or the main portal vein was seen in $58(19.2 \%)$ patients.

Not all cirrhotic patients develop HCC, and factors that define those at high risk include; male sex, age older than 50 years, increased alpha-fetoprotein (AFP) concentration, and intense inflammation and hepatocyte proliferation. The annual risk of HCC among patients with cirrhosis and raised serum AFP $(\geq 20 \mathrm{ng} / \mathrm{ml}$ ) is about $9.5 \%$. However, the sensitivity and specificity of AFP for diagnosing HCC varies range from $52 \%$ to $80 \%$ and $90 \%$ to $98 \%$. Another serum marker for HCC is des-gamma-carboxy prothrombin, also known as PIVKA-II (proteins induced by vitamin $\mathrm{K}$ absence or antagonist-II). It is highly sensitive (92\%) and specific (93\%) in differentiating early HCC from cirrhosis. PIVKA II levels greater than $0.1 \mathrm{AU} / \mathrm{ml}(100 \mathrm{ng} / \mathrm{ml})$ on ELISA are highly suggestive of HCC or tumor recurrence.

Cancer chemoprevention involves the use of either natural or synthetic chemicals to prevent the initiation, promotion, or progression of cancer. Prospective studies have been performed to evaluate the chemopreventive properties of antiviral agents such as interferon (IFN). Pegylated IFN and ribavirin therapy is highly effective in achieving sustained viral response (SVR) in HCV patients with cirrhosis of liver. Once SVR is achieved, the chances of HCC development are several fold reduced. Similarly, for HBV, use of antiviral agents like lamivudine and adefovir have been shown to achieve virus negativity and a reduction in the development of HCC.

Vitamin K2 has been similarly used as an agent to suppress tumor development. However, a recent trial has not found use of Vitamin K2 to be able to reduce frequency of tumor regression. Our group has earlier shown that Vitamin K3 can help in decreasing the rate of growth of HCC and even management of portal vein thrombosis in patients with advanced HCC. Numerous in vitro and in vivo studies have shown that vitamin D potently inhibits cell proliferation in a wide range of cell types, including carcinomas of the breast, prostate, colon, skin, and brain, myeloid leukemia cells, and others. Whether, it inhibits tumor development, progression or invasion is not known. Acyclic retinoid has been effectively tested in a single positive randomized controlled trial after surgical resection, but this approach is not considered standard of care. Further, its safety following long term use is uncertain.

With increasing incidence of HCC developing in patients with diabetes, obesity and alcoholic liver disease, there is a need to understand the pathogenetic role of metabolic pathways causing hepatocarcinogenesis. Phosphodiaesterase inhibitors like coffee have been shown to be highly protective in development of HCC in this group of patients.

Conclusion: HCC is a major complication of cirrhosis and usually presents at an advanced stage at the time of diagnosis in the Asian countries. Physicians caring for patients at risk in our countries need to provide high-quality screening, proper management of screen-detected lesions, and provision of therapy that is most appropriate for the stage of disease. There is a need for exploring new strategies for prevention of development of HCC in the Asian region. 


\section{Curriculum Vitae}

$\begin{array}{ll}\text { Name } & \text { Laurentius A. Lesmana } \\ \text { Position } & \text { Lecturer } \\ \text { Institution } & \text { University of Indonesia } \\ \text { E-mail } & \text { Llesmana.id@gmail.com }\end{array}$

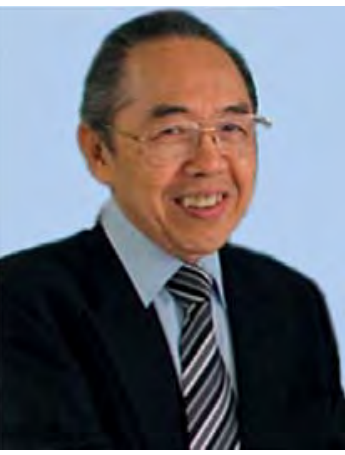

Dr. Laurentius Lesmana is Professor in Internal Medicine and Consultant Internist - Gastroenterohepatologist at the Department of Medicine, University of Indonesia, Jakarta. He graduated from the University of Indonesia in 1969 and became Consultant of Gastroenterohepatology at the same university in 1985. He received his $\mathrm{PhD}$ degree from the University of Amsterdam, the Netherlands in 1989.

Professor Lesmana received Fellow of the American College of Gastroenterology (FACG) in 1999 and Fellow of the American College of Physicians (FACP) in 2001.

He chaired the first annual APASL (Asian Pacific Association of the Study of the Liver) Conference 2005 in Bali, the first China-Indonesia joint meeting (CISHMS) in 2007 in Bali, and the first APASL Single Topic Conference on Hepatocellular Carcinoma in 2008 also in Bali. He is a reviewer of Acta Medica Indonesiana and Hepatology International journals. 


\section{Hepatocellular Carcinoma in Southeast Asia: Current Issues and Challenges}

Laurentius A. Lesmana

Department of Medicine, University of Indonesia, Digestive Disease Centre, Medistra Hospital, Jarkarta, Indonesia

Hepatocellular carcinoma (HCC) is a major health problem in Southeast Asia. Based on GLOBOCAN 2008 data, liver cancer is the third most commonly diagnosed cancer and the second cause of cancer death among the Association of Southeast Asian Nations (ASEAN), which include Brunei, Cambodia, Indonesia, Laos, Malaysia, Myanmar, the Philippines, Singapore, Thailand and Viet Nam. The highest incidence rates per 100,000 population are found in Laos $(33,8)$, Thailand (29.7), and Viet nam (29.3); while the lowest rates was found in Indonesia (6.7), Malaysia (5.7) and Brunei (5.2). The incidence in men is more than twice than in women.
The high prevalent chronic hepatitis B virus (HBV) infection in this region certainly has a causative role, but it is expected to decrease due to the routine hepatitis $\mathrm{B}$ vaccination among newborns. Chronic hepatitis $\mathrm{C}$ infection is the second common cause of HCC. Alcohol consumption is not considered as an etiological factor in Indonesia; however, it may be an issue in other ASEAN countries like Thailand, Malaysia (among Indian Malay), and Viet Nam. Smoking has been recently found as an independent risk factor for HCC in Singapore.

Recently, non-alcoholic fatty liver disease (NAFLD) has been associated with HCC development in developed countries. However, prospective studies evaluating NAFLD as a risk factor for HCC in Southeast Asia are still lacking. Data from Medistra Hospital between 2007 and 2012 showed that among 150 cases of HCC, there were 38 (25.3\%) with nonviral hepatitis or other cause of HCC. Type 2 diabetes was observed in almost half of them (47.4\%). Given the increasing incidence of fatty liver worldwide, the potential role of NAFLD as HCC risk factor should be alarming. 


\section{Curriculum Vitae}

$\begin{array}{ll}\text { Name } & \begin{array}{l}\text { Ji-Dong Jia } \\ \text { Position }\end{array} \\ & \begin{array}{l}\text { Professor of Medicine Director, } \\ \text { Liver Research Center } \\ \text { Institution }\end{array} \\ & \begin{array}{l}\text { Beijing Friendship Hospital, Capital } \\ \text { Medical University, Beijing, China }\end{array} \\ \text { E-mail } & \text { jia_jd@ccmu.edu.cn }\end{array}$

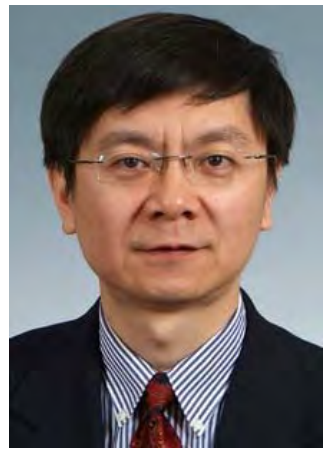

Ji-Dong Jia is a Hepatologist and Director of the Liver Research Centre at the Beijing Friendship Hospital, Capital University in Beijing, China. He is also immediate past President of the Chinese Society of Hepatology, Past President of APASL (2009-2010), Vice President of IASL. Dr Jia also serves as associate editor for Journal of Gastroenterology \& Hepatology, Hepatology International and Liver International. His clinical interests include the chronic viral hepatitis, autoimmune liver diseases. His research work mainly on cellular and molecular biology of the extracellular matrix of the liver and the pathogenesis and therapeutic study of experimental liver fibrosis.

\section{Research Interest:}

Cellular and molecular biology of the extracellular matrix of the liver and the pathogenesis and therapeutic study of experimental liver fibrosis. 


\section{Current Issues and Challenges for Controlling HCC in East Asia: Korea, Japan, China Mainland, Hong Kong and Taiwan}

\section{Ji-Dong Jia}

Liver Research Center, Beijing Friendship Hospital, Capital Medical University, Beijing, China

Hepatocellular carcinoma (HCC) accounts for between 85 and $90 \%$ of primary liver cancer and is one of the most frequent malignancies and the third-leading cause of cancer-related mortality worldwide. Regional efforts to control HCC should be adopted for greater political and public awareness and improved management of lifestyle risk factors.

In Korea, HCC is the third common leading cancer. During the last two decades, the incidence rate of HCC has shown a modest decrease, but its mortality rate has slightly increased. HBV represents approximately $70 \%$ and HCV represent approximately $20 \%$ of all HCC in Korea. Korea National Cancer Control Institute, which was reorganized in 2005, is now working for several national projects such as National Cancer Registration Program, National R\&D Program for Cancer Control and National Cancer Screening Program.

In Japan, the number of deaths from HCC increased until 2002 , and then started to decrease in 2003 , up to $15 \%$ of HCC cases are caused by HBV and approximately $80 \%$ by HCV infection. Fifteen percent of HCC was negative for hepatitis B surface antigen (HBsAg) and anti-HCV ((NBNC-HCC). HCV-HCC tended to decrease and NBNC-HCC tended to increase in recent years. Patients with NBNC-HCC and HCV-HCC were significantly older than those with HBV-HCC. The complication rates of diabetes mellitus (DM), heavy alcohol consumption, hypertension, and hyperlipidemia in NBNC-HCC were significantly higher than those in other groups. This trend suggests that $\mathrm{NASH}$ and metabolic syndrome were important risk factors for NBNC-HCC in Japan.

In Mongolia, the incidence of HCC is currently among the highest in the world. The mortality from HCC is also very high (52.2 deaths per 100,000 persons per year in 2010). The seroprevalence of HBV and HCV in general population in Mongolia is $11.8 \%$ and $15 \%$, respectively. One study showed that in patients with HCC, the seroprevalence of HBsAg, anti-HCV and dual infections was $50 \%, 27 \%$ and $21 \%$, respectively. Due to the lack of established surveillance systems, most cases of HCC are diagnosed at an advanced stage. To reduce the mortality from HCC in Mongolia implementation of HBV vaccination and safe injection as well as antiviral therapy is of paramount importance.

In China, HCC is the second most common cancer in urban areas and first most common in rural areas. It carries a mortality rate of 26.26 per 100,000 . Currently, people with HBV infection are a major population at risk of developing HCC in China. It is estimated that there are 93 million HBV carriers, and about 20 million of them are active chronic hepatitis B. Studies show that periodic surveillance of HCC with serum AFP and ultrasonography detect more early cases of HCC and reduce the mortality. There is an urgent need for a government-funded nationwide screening and surveillance program for high-risk patients and an evidence-based guideline for the management of HCC. 


\section{Curriculum Vitae}

$\begin{array}{ll}\text { Name } & \text { Ding-Shinn Chen } \\ \text { Position } & \begin{array}{l}\text { Distinguished Chair Professor } \\ \text { Institution }\end{array} \\ & \begin{array}{l}\text { National Taiwan University } \\ \text { College of Medicine } \\ \text { chends@ntu.edu.tw }\end{array}\end{array}$

I graduated from National Taiwan University College of Medicine in 1968, and completed clinical training in internal medicine and gastroenterology at the National Taiwan University Hospital in 1973. I joined the faculty of the Department of Internal Medicine, National Taiwan University College of Medicine as a lecturer in 1975. Before becoming Professor, I spent one year in National Institutes of Health, Bethesda, USA 1979-80. I was the Dean of National Taiwan University College of Medicine in 2001 07, and is Distinguished Chair Professor Since 2006.

I was elected Member of Academia Sinica (the National Academy of Taiwan) in 1992, Fellow of the Third World Academy of Sciences in 2001, and Foreign Associate, US National Academy of Sciences in 2005. I was the President of the Taiwan Association for the Study of the Liver (1996 98), the President of the Gastroenterological Society of Taiwan (1997 2003) and the President of the Formosan Medical Association (2001 2004). I served as the President of the International Association for the Study of the Liver (IASL) in 2004 2006. I earned many prestigious awards including those from EASL, APASL and AASLD.

\section{Research Interest:}

I have worked in the field of gastroenterology / hepatology for 40 years. My research interest is in basic and clinical aspects of viral hepatitis and liver disease (especially, HCC), ranging from prevention to management. 


\section{How to Control HCC in Asia \\ Ding-Shinn Chen}

Hepatitis Research Center, National Taiwan University

Hospital; Department of Internal Medicine, National

Taiwan University College of Medicine, Taipei, Taiwan

Hepatocellular carcinoma (HCC) is common in Asia, especially in East and Southeast Asia. Like other diseases, the control relies on prevention and treatment.

Because hepatitis B virus (HBV) and hepatitis $\mathrm{C}$ virus (HCV) are the two main etiologic factors of HCC, accounting for $\sim 80 \%$ of HCC in Asia, prevention of new infections and treatment of existing infections are mainstays of the prevention of HCC.

For primary prevention of HCC, subjects not infected by HBV should be immunized in early childhood. Results from Taiwan showed that after the implementation of hepatitis B mass vaccination in infants after 1984, the incidence of HCC had declined. In 6 9-year-olds, it declined from $0.49 / 100,000$ to $0.16 / 100,000$, in $10 \sim 14$-year-olds from $0.56 / 100,000$ to $0.21 / 100,000$, and in $15 \sim 19$-year-olds from $0.61 / 100,000$ to $0.20 / 100,000(p<0.001)$. The decrease of HCC will soon extend to young adults. For hepatitis $\mathrm{C}$, there is no effective vaccine. Interruption of the routes of transmission is important. New infections can be prevented by screening HCV in blood donors and by avoiding unsterilized mucocutaneous disruptions. In case of acute HCV infection, interferon-a should be given to prevent chronicity. In non-virally induced HCC, exposure to environmental hepatocarcinogens, such as excessive alcohol intake or fungal toxin contamination of food, should be avoided.
For secondary prevention of HCC, patients with chronic hepatitis $\mathrm{B}$ and $\mathrm{C}$ should be treated with effective antiviral therapy. There is convincing evidence that effective therapy to control HBV infection or eradicate HCV greatly reduces the occurrence of HCC. For areas where HBV and HCV are less prominent, non-alcoholic steatohepatitis may play an important role in causing HCC, life-style modification with diet control and increased physical exercise is needed.

For tertiary prevention, curative therapy for HCC should be accompanied with effective antiviral therapy in patients with HBV or HCV-induced HCC.

Outcomes of the treatment of HCC depend on the stages of the disease. HCCs in early stages are much more likely curable. They can only be detected in screening and surveillance of subclinical liver disease patients at risk of HCC. Therefore, hepatic ultrasonography and serum a-fetoprotein should be monitored regularly in those with cirrhosis, regardless of etiology, as well as those with chronic hepatitis B or C.

For advanced HCC, transarterial embolization and intraarterial chemotherapy can yield some beneficial results. In far-advanced patients, sorafenib, a small molecular multikinase inhibitor, has shown some positive results. However, the survival benefit is limited in Asian patients.

The control of HCC apparently needs a clear and strong government policy and support. The medical profession, especially the hepatologists, has to be active in persuading their own governments in forming a policy to control HCC. In the meantime, we need to train an adequate number of healthcare givers to implement the policy once it is formed. Hopefully, HCC can be controlled in Asia in several decades. 


\section{Curriculum Vitae}

Name

Position

Institution

E-mail
Jeong Won Jang

Associate Professor

The Catholic University of Korea

garden@catholic.ac.kr

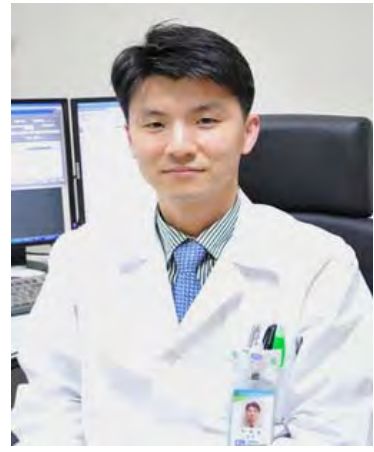

Dr. Jang is an Associate Professor of Internal Medicine at The Catholic University of Korea. He received his medical degree from The Catholic University of Korea, and completed his Internal Medicine Residency and Hepatology/ Gastroenterology Fellowship at Seoul St. Mary's Hospital in Seoul. He has been a faculty member of The Catholic University of Korea since 2005, and has been working at affiliated hospitals, Incheon St. Mary's Hospital (2005-2012) and Seoul St. Mary's Hospital (2013 present). His current clinical activities include attending the Gastroenterology service at Seoul St. Mary's Hospital and caring for patients with liver diseases at a hepatology unit. He is currently a board member of the Korean Association for the Study of Liver Diseases and The Korean Liver Cancer Study Group. He has authored and co-authored over 80 articles and one book.

\section{Research Interest:}

Dr. Jang's clinical expertise includes viral hepatitis and liver cancer. His areas of research interest include (1) the reactivation and immunology of hepatitis B, (2) mutation and carcinogenesis of hepatitis B virus, and (3) inflammatory process in hepatocarcinogenesis. 


\section{How to Control HBV-Related HCC \\ Jeong Won Jang \\ Catholic University Liver Research Center, Department of Internal Medicine, The Catholic University of Korea, Seoul, Korea}

Chronic infection with hepatitis B virus (HBV) is a major risk factor for the development of hepatocellular carcinoma (HCC) in Asian countries. Viral factors that have been associated with an increased risk of HCC include hepatitis B e-antigen (HBeAg)-positive status, a high level of HBV DNA, molecular variants in the viral genome such as basal core promoter mutations (BCP T1762/A1764), HBx expression, genotype $\mathrm{C}$, and integration of HBV DNA into the host genome. The most important preventive measure against HBV-related HCC is the universal hepatitis B vaccination, which has previously been adopted in several Asian countries since 1980s. Indeed, the implementation of universal vaccination has shown a clear reduction in the prevalence of chronic hepatitis $B$ as well as HCC in children in Taiwan.

Recently, accumulating evidence from prospective studies has indicated that an increased level of serum HBV DNA is a key predictor of HCC, irrespective of status for HBeAg and liver cirrhosis. For chronically infected persons, antiviral therapy remains the only option to control HCC development. One randomized controlled study and other cohort studies have shown that interferon (IFN) treatment decreases the risk of HCC, particularly in patients with liver cirrhosis who achieved sustained viral suppression with therapy. However, further studies are needed to have convincing evidence supporting for the preventive role of IFN, because there is significant heterogeneity in patient characteristics, disease stage and study design among those studies. Maintained suppression of HBV DNA with oral nucleos(t)ide analogs (NUC) has also been associated with a lower risk of HCC development. A large randomized controlled trial of lamivudine in patients with advanced fibrosis and cirrhosis has revealed that lamividine therapy significantly reduced overall disease progression and HCC development as compared to control subjects, although the benefit of lamivudine was blunted by the development of drug resistance. A meta-analysis and systematic reviews have concluded that treatment with NUC is associated with a lower risk of HCC compared to untreated subjects.

Preventive measures should also been done even in patients who are diagnosed with HBV-related HCC. Similar to settings of other malignancies, HBV reactivation has often been observed in patients with HCC undergoing anti-cancer therapy, sometimes leading to hepatic decompensation in a subset of patients. Systematic reviews demonstrated that preemptive antiviral therapy before the start of chemotherapy reduces HBV reactivation in patients receiving anti-cancer therapy. Thus, the current practice guidelines recommended that antiviral drugs should be initiated before cancer chemotherapy. This approach plays a role in not only reducing the risk of HBV reactivation but also preserving against liver functional deterioration that can be caused by HBV activity during therapy, facilitating continued anti-cancer therapy, without compromising planned treatment sessions.

In conclusion, hepatitis $\mathrm{B}$ vaccination is the most powerful measures as a primary prevention for HBV-related HCC. IFN seems to have a modest beneficial effect on reducing HCC risk, but the evidence is not strong. Oral antiviral therapy significantly reduces but not abolishes the risk of HCC in cirrhotic patients. There is still a need for well-designed, large prospective studies to confirm the definitive role of antiviral therapy in HCC risk in patients with chronic HBV infection. 


\section{Curriculum Vitae}

$\begin{array}{ll}\text { Name } & \text { W. Ray Kim } \\ \text { Position } & \text { Professor of Medicine } \\ \text { Institution } & \text { Mayo Clinic } \\ \text { E-mail } & \text { kim.ray@mayo.edu }\end{array}$

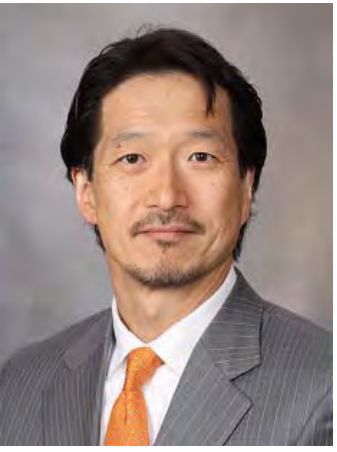

Dr. Kim received his medical degree at the Seoul National University in Seoul, Korea. He then moved to the US where he attended the Wharton School of Business for an MBA degree in Healthcare Administration. His postgraduate training included Internal Medicine at Seoul National University Hospital and University of Arkansas for Medical Sciences, as well as Gastroenterology at Mayo Clinic in Rochester, MN. He also completed a subspecialty fellowship in Hepatology and Liver Transplantation at Mayo Clinic Rochester. His clinical expertise includes end stage liver disease and viral hepatitis. He is currently an Associate Editor of Hepatology and Chair of Clinical Research Committee of the American Association for the Study of Liver Diseases.

\section{Research Interest:}

Dr. Kim is currently on faculty at Mayo Clinic College of Medicine, where he is a Professor of Medicine. He has active research programs, mostly supported by US federal grants, including studies of (1) outcome of liver transplantation, (2) prevention of renal insufficiency in liver transplant recipients, (3) outcome and treatment of hepatitis B and (4) methods of screening and diagnosis of liver disease in the population. His research program is credited for the development of the MELD (model for end stage liver disease), which has been adopted in the US and elsewhere in the world as a standard by which donated livers are allocated. 


\section{Strategies to Control Hepatocellular Carcinoma in the US}

W.R. Kim

Mayo Clinic, Rochester, MN, USA

In the United States, liver and intrahepatic bile duct cancers are the fifth most common cause of cancer death in men and ninth in women. For 2013, 21,670 Americans are expected to die from these cancers, including 14,890 men and 6,780 women. The number of Americans who develop liver cancers continues to increase. Although the reason for the increase is believed to be multifactorial, the most important factor is believed to be chronic hepatitis C virus (HCV) infection. As the generation of Americans with high prevalence of HCV grows older with increasing duration of infection, it is projected that the incidence of cirrhosis, hepatic decompensation and HCC will continue to rise in the foreseeable future. Another potentially important cause of liver cancer that may underlie the recent increase in HCC incidence is obesity and insulin resistance leading to non-alcoholic fatty liver. However, the extent to which non-alcoholic fatty liver is contributing to the epidemiological trend in liver cancer in the US remains to be defined.

For effective control of liver cancer in a population, multifaceted primary, secondary and tertiary prevention efforts are required. Primary prevention refers to measures to decrease the incidence of HCC. The most fundamental primary prevention would be to reduce the prevalence of underlying chronic liver disease, which predisposes the patient to HCC. While the best known example for this may be immunization against hepatitis B in Taiwan, given the low prevalence of HBV in the US, the universal vaccination program started in the early 1990s is expected to have a modest impact. On the other hand, efforts to curtail further spread of HCV and more importantly, treat HCV infected individuals before they develop advanced liver disease is likely to have a much larger impact. To date, however, population-wide efforts to screen indi- viduals for HCV has not been widely accepted and even in patients diagnosed with HCV, applicability and effectiveness of interferon-based antiviral treatment has been limited. As substantial progress in HCV therapy is expected in the near future, widespread screening and diagnosis followed by viral eradication may have a large impact on HCC incidence. Lastly, chemoprevention remains an attractive yet elusive approach to date to reduce the incidence of HCC in at-risk individuals.

Secondary prevention refers to diagnose and treat HCC in early stages before it causes significant morbidity. While literature evidence to support that surveillance for HCC reduces mortality is modest, the AASLD guideline for HCC recommends surveillance to be conducted in select patients with liver disease. The criteria for selection of candidates for HCC surveillance are derived from cost-effectiveness analyses. The threshold incidence to warrant surveillance is set at $0.2 \%$ per year for non-cirrhotic hepatitis B patients and at 1.5\% per year for patients with cirrhosis from other etiologies. There have been a number of mathematical models published recently to estimate the risk of HCC development. Although they are mostly for hepatitis B patients, accurate risk assessment tools have a substantial potential to improve the effectiveness of HCC surveillance. Finally, sensitive and accurate biomarkers are urgently needed.

Tertiary prevention purports to minimize the negative impacts of HCC by reducing disease-related complications, minimize the risk of recurrence after treatment and restore the function and well-being of the patient. This truly requires multidisciplinary efforts not only from a team of physicians, surgeons, and interventional radiologists, but also from nurses, social workers, dieticians and other support personnel. The BCLC staging system remains the widely adopted roadmap to implement tertiary prevention for HCC patients. Because of heterogeneity in the tumor biology and lack of universally effective pharmacological agents, universally effective tertiary chemoprevention is not available. Recent data indicate that for hepatitis B patients, antiviral therapy is associated with reduced recurrence after surgery. 
The 4th Asia-Pacific Primary Liver Cancer Expert Meeting

\section{Curriculum Vitae}

Name

Position
Masayuki Kurosaki

Director, Department of

Gastroenterology and Hepatology, Musashino Red Cross Hospital Associate Professor, Tokyo Medical and Dental University Assistant Professor, Yamanashi University

Institution E-mail

Musashino Red Cross Hospital kurosaki@musashino.jrc.or.jp

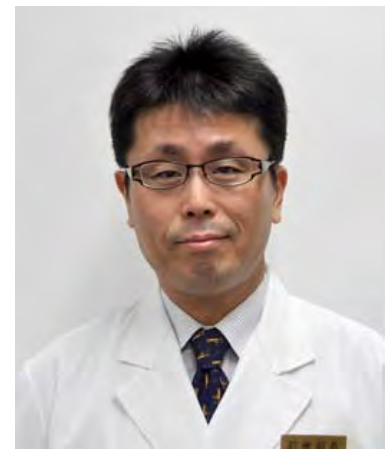

\section{Academic Qualifications:}

1995, Ph.D. in Medicine, Tokyo Medical and Dental University 1987, M.D. in Medicine, Tokyo Medical and Dental University

\section{Working/ Teaching Experience:}

2011-present, Assistant Professor, Yamanashi University 2010-present, Director, Department of Gastroenterology and Hepatology, Musashino Red Cross Hospital 2006-present, Associate Professor, Tokyo Medical and Dental University 2003-2010, Associate Director, Dept. of Gastroenterology and Hepatology, Musashino Red Cross Hospital

1995-2003, Assistant Professor, Tokyo Medical and Dental University

\section{Professional Membership:}

Councilor and fellow of Japan Society of Hepatology

Councilor and fellow of the Japanese Society of Gastroenterology

Councilor and fellow of Japan Gastroenterological Endoscopy Society

Fellow of the Japanese Society of Internal Medicine

\section{Editorial Board:}

Present, BioMed Research International present, World Journal of Hepatology

\section{Research Activities:}

Co-investigator of 6 Study group supported by Ministry of Health, Labour and Welfare, Japan

Research Interest:

Hepatitis B and C

Hepatocellular Carcinoma 


\section{How to Control HCV-related HCC: A Personalized Strategy}

\author{
Masayuki Kurosaki \\ Musashino Red Cross Hospital, Tokyo, Japan
}

HCC risk prediction model revealed that HCC risk reduction by interferon therapy differ by the baseline risk

The annualized rate of developing hepatocellular carcinoma (HCC) from type C liver cirrhosis is $2 \%-8 \%$ which could be reduced by interferon (IFN) therapy. However, the annualized rate of HCC development is $<1.5 \%$ in patients with chronic hepatitis $\mathrm{C}$ but without cirrhosis. Whether the incidence of HCC in these patients could be reduced by IFN remains to be elucidated.

To solve these problems, we have builtan HCC risk prediction model by data mining analysis in a cohort of chronic hepatitis $\mathrm{C}$ patients without cirrhosis (Kurosaki et al. Journal of Hepatology, 2012). Data mining analysis is performed in an exploratory manner to extract relevant factors and to combine them in an orderly sequence to identify rules for predicting the incidence of the target outcome. On the basis of factors such as age, platelet, albumin, and AST, the HCC risk prediction model identified subgroups with high-, intermediate-, and low-risk of HCC with a 5-year HCC development rate of $20.9 \%, 6.3 \%-7.3 \%$, and $0 \%-1.5 \%$, respectively. In the high and intermediate-risk group, the incidence of HCC development was significantly reduced in patients with sustained virological response (SVR) than those with non-SVR (5-year rate, 9.5\% vs. $4.5 \%$; $P=0.040$ ). On the other hand, in the low-risk group, the 5-year rate was 1.8\% in non-SVR patients and $0.9 \%$ in SVR patients. Both rates were low and not significantly different $(P=0.331$, log-rank test). According to this model, patients at high risk of HCC should be treated to prevent future HCC development.

Assessment of short-term HCC risk for considering the timing of antiviral therapy in the era of rapidly evolving HCV therapy

The rate of SVR has significantly increased by the introduction of telaprevir, a highly effective direct antiviral agent (DAA) but with frequent adverse events (AE). In addition, numerous newer DAAs with higher efficacy, lower rate of AE, and shorter treatment duration are expected to be clinically available within several years. In this era of rapidly evolving HCV therapy, most physicians and patients prefer to wait for newer DAAs if the risk of HCC development within few years is negligible. Another HCC prediction model we have built is for the prediction of HCC development within 2 to 3 years. Factors selected finally were alpha-fetoprotein (AFP) and platelet. The risk of HCC development in patients with AFP $\geq 3.9 \mathrm{ng} / \mathrm{mL}$ and platelet $<150 * 10^{9} / \mathrm{L}$ was $3.9 \%$ at year 2 and $7.2 \%$ at year 3 , while it was $0.3-0.6 \%$ and $0.5-0.8 \%$, respectively in other patients. Again, the risk of HCC was significantly reduced by IFN therapy in the high- risk group but not in the low-risk group. Thus, risk assessment could be used to identify patients who benefit from immediate therapy and those who could wait for newer emerging therapy.

Possible surrogate markers for HCC risk reduction in patients who fail to achieve SVR

Relevant factors for HCC development was extracted from 975 patients who failed to achieve SVR by IFN therapy: older age
(HR for $>65$ yrs: 3.12 ), low platelet counts (HR for $<150^{*} 10^{9} / \mathrm{L}$ : 2.78), high AFP (HR for $>10 \mathrm{ng} / \mathrm{mL}: 2.06$ ), and high ALT (HR for $>40$ IU/L: 2.27). While age and platelet counts are unchangeable, AFP and ALT could be reduced by IFN therapy. In older patients ( $>65$ yrs) with ALT $>40$ IU/L and who failed to achieve SVR, ALT decreased to $<40$ in $42 \%, 45 \%, 59 \%$, and $68 \%$ of patients treated by IFN mono-therapy for 24 weeks, IFN plus ribavirin (RBV) for 24 weeks, peg-IFN mono-therapy for 48 weeks, and peg-IFN plus RBV for 48 weeks, respectively. In older patients with AFP $>10 \mathrm{ng} / \mathrm{mL}$, AFP decreased to $<10 \mathrm{ng} / \mathrm{mL}$ in $36 \%, 32 \%, 57 \%$, and $56 \%$, respectively according to the treatment regimen as described above. Thus, the longer duration and addition of RBV favored the normalization of ALT, and AFP despite failing to achieve SVR. In those who achieved ALT or AFP normalization, $73 \%$ and $78 \%$ remained to have normal ALT or AFP 3 years after the last dosage of treatment. Actually, normalization of ALT and AFP post-IFN significantly reduced the risk of HCC by HR of 0.41 and 0.44 , respectively.

There is a debate on a possible role of long term maintenance therapy with peg-IFN for the reduction of HCC risk. In our cohort of 163 patients treated by peg-IFN for more than 1 year, normalization of ALT and AFP was obtained in 51\% and 36\%, respectively. The risk of HCC was decreased in these patients (HR for ALT <40:.40 and for AFP <10: 0.32). These result indicated that patients at risk of HCC should be treated by IFN, even if the possibility of SVR seems low, in order to achieve a possible surrogate marker for HCC risk reduction. Extending the treatment duration may be warranted for patients with favorable reduction of ALT or AFP.

Identification of patients who need surveillance for HCC after successful eradication of HCV by antiviral therapy

Highly effective antiviral therapy will lead to increased number of patients with successful eradication of HCV. While the overall incidence of HCC is expected to decrease by increased rate of SVR, the number of HCC development after HCV eradication may paradoxically increase.

Multicenter study was conducted by Japanese Red Cross Liver Study Group, involving 18 hospitals and medical centers nationwide. A total of 785 genotype $1 \mathrm{~b}$ chronic hepatitis $\mathrm{C}$ patients who had successful eradication of HCV by IFN therapy were enrolled. The cumulative incidence of HCC development after HCV eradication was $4.0 \%$ in 3-yr, $7.1 \%$ in 5-yr, and $12.4 \%$ in $7-y r$, respectively. Age over 60 (HR 3.80), male (HR 3.54), platelet counts below $150 * 10^{9} / \mathrm{L}$ (HR 2.11), and serum AFP $>5 \mathrm{ng} / \mathrm{ml}$ at 24 weeks after the completion of therapy (HR 3.21) were independent risk factors for the development of HCC. The 5 year incidence of HCC in patients with AFP levels of $<5,5-9,10-19$, and $\geq$ $20 \mathrm{ng} / \mathrm{ml}$ was $3.7 \%, 12.2 \%, 21.3 \%$, and $34.5 \%$ respectively. Patients with these hallmarks of residual risk for HCC development may be the candidate for a careful HCC surveillance even after the complete eradication of hepatitis $C$ virus.

Antiviral therapy after the curative loco-regional ablation therapy for HCC

There is no solid evidence for supporting IFN therapy for the prevention of HCC recurrence after curative treatment of HCC. Multicenter PRAHT study (PEG-IFN·RBV After HCC Treatment) revealed that cumulative incidence of HCC recurrence was not reduced by SVR but was significantly reduced by normalization of AFP (HCC recurrence rate at year 3 for AFP $<10$ vs. $>10$ were $23.1 \%$ vs. $61.5 \%$ ). The role of IFN therapy for the prevention of HCC recurrence needs further investigation. 
The 4th Asia-Pacific Primary Liver Cancer Expert Meeting

\section{Curriculum Vitae}

Name

Position

\section{Seng Gee Lim}

Chairman of the Asia Pacific

Association for Study of the

Liver (APASL) Liver Week

2013 Congress

Institution

Dept. of Gastroenterology and

Hepatology, National University

Health System, National University

of Singapore

seng_gee_lim@nuhs.edu.sg

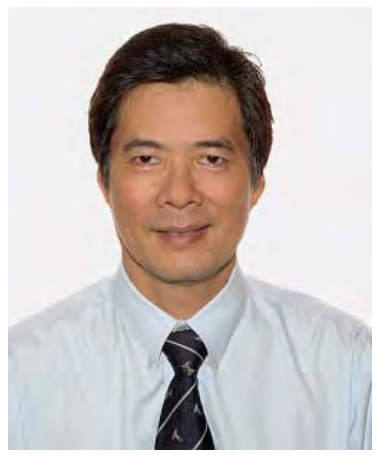

Professor Seng Gee Lim, FRACP, FRCP, FAMS, MD, was the Chairman of the Asia Pacific Association for Study of the Liver (APASL) Liver Week 2013 Congress. He is currently director of Hepatology, in the Dept. of Gastroenterology and Hepatology National University Health System where he is Professor of Medicine in the Yong Loo Lin School of Medicine, National University of Singapore.

Dr. Lim attained his basic medical degree (MBBS) with honors from the University of Monash, Melbourne, Australia. He later pursued a doctorate degree (MD) in Monash and was awarded the FRACP (Australia), FRCP (London), and FAMS (Gastroenterology, Singapore).

His main clinical work focuses on treatment of viral hepatitis (especially B and C), liver disease, and liver cancer. Dr Lim's research activities include leadership in Hepatology research, which focuses on improving treatment for chronic hepatitis B and C, pathophysiology of hepatitis B disease, HBV virology and immunology, as well as drug related liver disease, non-alcoholic fatty liver disease and hepatocellular cancer.

With over 180 publications in peer review journals, Dr Lim sits on the editorial board of Liver International, Journal of Viral Hepatitis, Hepatology International, Alimentary Pharmacology and Therapeutics, Evidence Based Internal Medicine Solutions, Open Tropical Medicine Journal. and is a reviewer for Singapore's grant funding agencies. He is an advisory board member for Novartis Pharmaceuticals, Bristol Myers Pharmaceuticals, Merck Sharpe and Dohme Pharmaceuticals, Boehringer Ingelheim Pharmaceuticals, Gilead Pharmaceuticals, Pfizer Pharmaceuticals, Janssen Pharmaceuticals, and Archillion Pharmaceuticals. 


\section{How to Control HCC in the Asia Pacific - APASL Policy to control}

Seng Gee Lim

Dept. of Gastroenterology and Hepatology, National University Health System, Yong Loo Lin School of Medicine, National University of Singapore, Singapore

Recently the Global Burden of Disease study 2010 found that hepatocellular carcinoma (HCC) mortality increased to 752,000 deaths from 463,000 in 1990 . Of these 341,000 was due to hepatitis B, while hepatitis C contributed 195,000 and alcohol 143,000 . Other causes only contributed 66,000 . As hepatitis B is the main cause of HCC, the best prevention measure is universal hepatitis $B$ vaccine. This has been widely implemented across Asia for over 25 years but there is still a large burden of infected patients who are over 25 years old. Nonetheless, childhood HCC has been significantly reduced as a result of vaccination. Since treatment of chronic hepatitis $B$ and cure of hepatitis $C$ reduce the development of HCC, the focus of efforts to reduce HCC in the Asia Pacific should focus on treatment of chronic viral hepatitis. In this regard, the WHO resolution in 2010, followed by the Global Hepatitis Program and now the launch of the WHO Global Hepatitis Network during APASL 2013 Liver Week. A concerted effort by all stakeholders including WHO, governments, non-governmental organisations, patients, physicians, pharmaceutical companies and liver associations are needed to combat viral hepatitis and subsequently control HCC. 


\begin{tabular}{l|l}
\hline DOI: $10.1159 / 000343846$ & $\begin{array}{l}\text { (C) 2013 S. Karger AG, Basel } \\
\text { www.karger.com/lic }\end{array}$ \\
\hline
\end{tabular}

The 4th Asia-Pacific Primary Liver Cancer Expert Meeting

\section{Curriculum Vitae}

$\begin{array}{ll}\text { Name } & \text { Tetsuya Hosaka } \\ \text { Position } & \text { Staff clinician } \\ \text { Institution } & \begin{array}{l}\text { Toranomon Hospital } \\ \text { Dept. of hepatology } \\ \text { hosa-p@toranomon.gr.jp }\end{array}\end{array}$

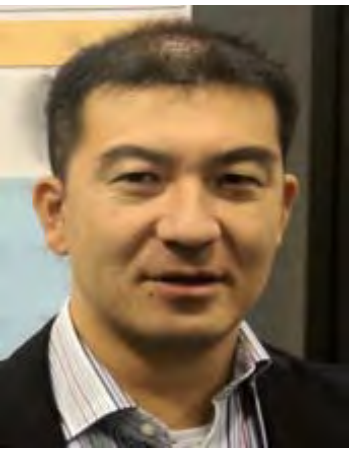

Education:

1998 Osaka University, Medical School

Licensure and Certification:

National Board of Medicine, Registration No. 395348

Board Certified Hepatologist of the Japan Society of Hepatology No. 4328

Board Certified Gastroenterologist of the Japanese Society of Gastroenterology No. 28951

Board Certified Endoscopist of Japan Gasteroenterological Endoscopy Society No. 20100407

Fellowship:

1998/May -1999/ May Study in Osaka University (Internal Medicine)

\section{Memberships:}

Japan Society of Hepatology

Japanese Society of Gastroenterology

Japan Gasteroenterological Endoscopy Society

\section{Honors and Awards:}

Academic Encouragement Award of the Japanese Society of Gastroenterology 2008 Academic Encouragement Award of the Japan Society of Hepatology 2010

\section{Research Interest:}

Clinical research: Viral hepatitis and hepatocellular carcinoma

Biostatistics

\section{Publications: (in the Last 5 Years)}

First author

1 Hosaka T, Suzuki F, Kobayashi M, et al. Long-term entecavir treatment reduces hepatocellular carcinoma incidence in patients with hepatitis B virus infection. Hepatology, 2012 (Epub ahead of print).

2 Hosaka T, Suzuki F, Kobayashi M, et al. Clearance of hepatitis B surface antigen during long-term nucleot(s)ide analog treatment in chronic hepatitis B: results from a nine-year longitudinal study. J Gastroenterol, 2012 (Epub ahead of print).

3 Hosaka T, Suzuki F, Kobayashi M, et al. HBcrAg is a predictor of post-treatment recurrence of hepatocellular carcinoma during antiviral therapy. Liver Intl 2010;30:1461-1470.

4 Hosaka T, Suzuki F, Kobayashi M, et al. Development of HCC in patients receiving adefovir dipivoxil for lamivudine-resistant hepatitis B virus mutants. Hepatol Res 2010;40:145-152.

5 Hosaka T, Ikeda K, Kobayashi M, et al. Predictive factors of advanced recurrence after curative resection of small hepatocellular carcinoma. Liver Intl. 2009;29:736-742. 


\section{Antiviral Therapy to Prevent HCC Development}

Tetsuya Hosaka

Toranomon Hospital, Dept. of Hepatology, Tokyo, Japan

Background: Chronic hepatitis B virus (HBV) infection leads to cirrhosis and hepatocellular carcinoma (HCC). Antiviral agents are thought to reduce HCC development, but agent such as lamivudine has a high rate of drug resistance.

Method: We compared the incidence of HCC in 472 entecavir (ETV)-treated patients and 1143 non-treated HBV patients (control group). Propensity score matching eliminated the baseline differences, resulting in a sample size of 316 patients per cohort.

Results: The drug mutation resistance was $0.8 \%$ (4/472) in the ETV group. The cumulative HCC incidence rates at 5-year were $3.7 \%$ and $13.7 \%$ for the ETV and control groups, respectively $(P<0.001)$. This difference was found in cirrhotic patients $(P<0.001)$ but not in non-cirrhotic patients $(P=$ 0.440 ). Cox proportional hazard regression analysis, adjusted for a number of known HCC risk factors, showed that patients in the ETV group were less likely to develop HCC than those in the control group (hazard ratio: $0.37 ; 95 \% \mathrm{CI}: 0.15$ to $0.91 ; P=$ 0.030 ). Both cohorts were applied into 3 previously reported risk scales and risk scores were generated based on age, gender, cirrhosis status, levels of ALT, HBeAg, baseline HBV DNA, albumin, and bilirubin. The greatest HCC risk reduction occurred in high-risk patients who scored higher on respective risk scales.

Conclusion: Long-term entecavir treatment may reduce the incidence of HCC in HBV-infected patients. The treatment effect was greater in patients at higher risk of HCC. 


\section{Curriculum Vitae}

$\begin{array}{ll}\text { Name } & \text { Pei-Jer Chen } \\ \text { Title } & \text { Professor } \\ \text { Affiliation } & \text { Graduate Institute of Clinical } \\ & \text { Medicine, National Taiwan } \\ & \text { University and Hospital } \\ \text { Email } & \text { peijerchen@ntu.edu.tw }\end{array}$

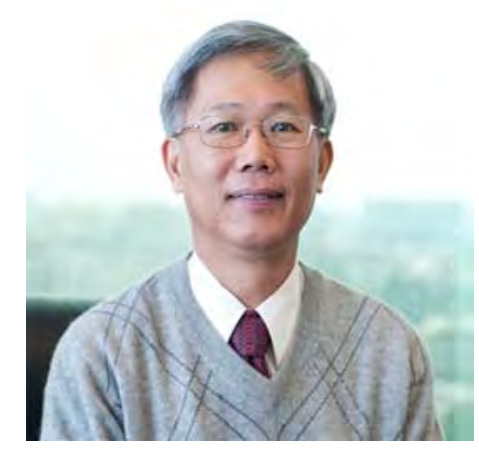

Professor Chen is the President of Taiwan Association for Study of the Liver (TASL) from 2012 to 2013. He was appointed Director of the Hepatitis Research Center at the National Taiwan University Hospital in Taipei in 2001-2003, and now the faculty for Graduate Institute of Clinical Medicine, National Taiwan University. His research interests focus on the molecular virology of hepatitis viruses, and the genetic and genomic study of hepatocellular carcinoma. Professor Chen's clinical research covers the natural history of chronic viral hepatitis and hepatocellular carcinoma, and also explores and conducts new therapies and trials for both diseases.

From 2001 to 2006, he was a member of the editorial board of the journal Hepatology and reappointed since year 2010-2011. In 2000, Professor Chen was awarded the International Research Scholar in Infectious Diseases by the Howard Hughes Medical Institute for a period of five years. He has been elected into Academia Sinica in year 2006 . He has published over 470 articles in the areas of hepatitis and hepatocellular carcinoma. 


\section{How to Prevent Early Recurrence after Curative Treatment of HCC}

\section{Pei-Jer Chen}

Hepatitis Research Center, National Taiwan University Hospital, Taipei, Taiwan

Curative surgery is the mainstream treatment for early stage HCC. Despite the clinico-pathological evidences of a complete removal of HCC, patients still suffers intrahepatic recurrence at a rate about $10-20 \%$ per year. The frequent recurrence limits the treatment efficacy and shortens the survival period. How to prevent HCC recurrence remains an unmet medical need at present.

For those recurrences taking place within one or two years after curative treatments, experimental studies have been shown their origins largely arising from the intrahepatic micrometastasis of the original HCC, that clinically undetectable at the time of treatment. Therefore the control of early recurrence likely has to eliminate or suppress the presence or growing of these spreading microscopic tumor foci. Along this line, anti-cancer interventions or drugs have been tried to prevent recurrences. TCAE and other interventions have been examined without conclusion. Recently, many new investigational drugs are tested in well-designed, largescale, randomized clinical trials, including Sorafenib (STORM trial), heparinase-inhibitor (PATRON trial) and others ongoing trials. The progress will be summarized in this presentation. In the near future, the results of these clinical trials will yield important insights and impact the adjuvant therapy for postcurative HCC. 


\section{Curriculum Vitae}

$\begin{array}{ll}\text { Name } & \text { Hyung Joon Yim } \\ \text { Position } & \text { Professor } \\ \text { Institution } & \begin{array}{l}\text { Division of Gastroenterology and } \\ \text { Hepatology } \\ \end{array} \\ & \begin{array}{l}\text { Department of Internal Medicine } \\ \text { Korea University Medical College }\end{array} \\ & \text { gudwns21@medimail.co.kr }\end{array}$

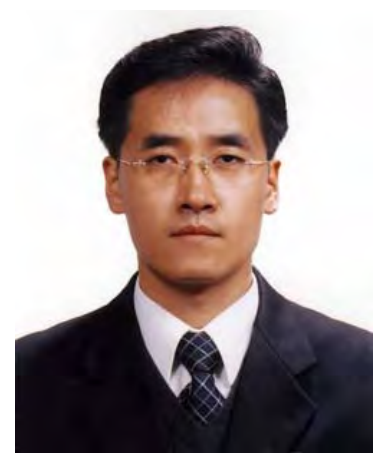

Dr. Hyung Joon Yim is Associate Professor of Division of Gastroenterology and Hepatology at Korea University Medical College, Ansan Hospital.

He graduated from Korea University Medical College and received Ph.D. of Internal Medicine from Korea University Graduate School. He completed the Research fellowship at Division of Gastroenterology, University of Michigan, USA.

Dr. Yim's research interests include acute and chronic hepatitis and virology as well as chemoprevention of hepatocellular carcinoma. His recent research focused on chronic hepatitis B and had experiences in various multicenter clinical trials, focused on chronic hepatitis B. He published hundred of papers untill now and actively participates as a reviewer of many international journals.

Currently, Dr. Yim serves as the chair of academic committee of the Korean Liver Cancer Study Group and a member of academic committee of the Korean Association for the Study of the Liver. 


\section{How to Prevent Late Recurrence after Curative Treatment}

Hyung Joon Yim

Department of Internal Medicine, Korea University

Ansan Hospital, 516 Gojan-dong, Danwon-gu,

Ansan-si, Gyeonggi-do, Korea

Hepatocellular carcinoma (HCC) is a highly lethal cancer and the third leading cause of cancer death world-wide. The prognosis of HCC is dismal unless the tumor is not treated in the early stage with curative intent. Curative therapies for HCC include hepatic resection, liver transplantation, and local ablative therapies. However, although the original tumor is completely removed, the remnant diseased liver is the source of new foci for recurrence of cancer. The recurrence rate is reported 50-70\% even after the complete surgical excision. Therefore, effective preventive strategy is needed after the curative treatment. Herein, brief review of current data will be done regarding prevention of recurrence, especially, with long term perspective.

\section{Risk Factors for Late Recurrence}

Recurrence can be outlined by early and late recurrences although distinctive time point cannot be provided. However, recurrence within 2 year of curative therapy is commonly suggested as early recurrence, and recurrence after 2 year is considered late recurrence. ${ }^{1}$ The risk factors for the recurrence and prognosis may be different between early and late recurrence of HCC. It has been postulated that early recurrence is associated with intrahepatic metastasis and late recurrence is developed most likely due to multicentric occurrences. ${ }^{2}$ Hence, the prognosis of the early recurrence is certainly worse. Previously, it has been reported that risk factors for early recurrence after resection are preoperative tumor rupture and venous invasion while presence of underlying cirrhosis is the sole independent factor for late recurrence. ${ }^{2}$ In other words, tumor factors but not host factors are linked to early recurrences, whereas only the non-tumorous liver status is linked to late recurrence. Cirrhosis is a well known risk factor for development of HCC, and the factors contributing progression of liver cirrhosis would promote late recurrence.

Approximately $70-80 \%$ of liver cirrhosis are related to hepatitis B virus (HBV) or hepatitis C virus (HCV) infection. It has been reported that active replication of these viruses is associated with increased risk of developing liver cancers. ${ }^{3,4}$ Also, high viral load increases the risk of HCC recurrence after resection in patients having underlying cirrhosis related to hepatitis $\mathrm{B}$ virus as well as hepatitis $\mathrm{C}$ virus infection. ${ }^{5,6}$ Increased hepatic inflammatory activity is also an independent risk factors for late recurrence in hepatitis B-related HCC. ${ }^{7}$ Likewise, elevated transaminases which reflects degree of necroinflmmation was associated with late recurrence of HCC in patients with HCV infection. ${ }^{5,8}$ These data suggest need of antiviral therapy in hepatitis virus associated HCC.
Recently, a number of clinical and basic research are undergoing to reveal the markers for the early and late recurrences after the curative therapies. Expression of cytokeratin 10 and cytokeratin 19 was suggested as markers for poor prognosis after resection, ${ }^{9}$ and high serum vascular endothelial growth factor before the curative therapy was also associated with recurrences of HCC. $^{10}$ Omics-based research is also focusing on chromosomal aberrations, epigenetic abnormality, and changes of gene expression to reveal molecular mechanism of HCC. MicroRNAs (miRNAs) are one of emerging candidates for markers of prognosis in HCC. ${ }^{11}$ It has been reported that the tumor miRNA profile could predict early recurrences, whereas the miRNA profile of the nontumoral tissue was predictive of late recurrences. ${ }^{12}$ Further research would be needed for wide application of these results in clinical practice.

\section{Antiviral therapies for the prevention of late recurrences}

\section{1. $H B V$-related $H C C$}

Current data suggest that high viral load is associated with recurrence of HBV-related HCC. ${ }^{4}$ A Korean study reported $73 \%$ of recurrence in viremic patients compared with $55 \%$ in non-viremic patients after curative resection $(p=0.043){ }^{6}$ Another study also reported viral load $>2,000 \mathrm{IU} / \mathrm{mL}$ is the most important correctable factor for HCC recurrence after resection (OR 22.3, 95\% CI 3.3-151, $\mathrm{P}=0.001$ ). ${ }^{13}$ Therefore antiviral therapy is suggested to be implemented in these patients.

Interferon was tried in HBV-related HCC patients after resection or ablation. ${ }^{14,15}$ Someya et al. reported significantly lower rate of recurrence in the interferon-treated group. Tumor recurrence rates in the treated group and untreated groups were $16.7 \%$ and $37.9 \%$ at the end of the first year, $16.7 \%$ and $60.1 \%$ at the second year, and $16.7 \%$ and $83.4 \%$ at the third year, respectively ( $\mathrm{P}=0.0139$ ), suggesting early as well as late recurrence was prevented. ${ }^{14}$ Multivariate analysis showed that interferon significantly decreased the recurrence rate $(\mathrm{OR} 0.21, \mathrm{P}=0.037)$. Another large randomized controlled trial showed significant improvement of overall survival in the interferon treatment group compared with the control group (63.8 months vs. 38.8 months, respectively, $\mathrm{P}=$ 0.0003). ${ }^{15}$ Recent meta-analysis regarding effect of interferon on survival and the recurrences of HBV-related HCC showed improved survival and reduced recurrence rates by interferon therapy, but the study was performed to estimate the effect at 2-years only. ${ }^{16}$

Nucleoside analogues are now widely used for the treatment of chronic HBV infection. The effect of these drug on prevention of HCC recurrence has been studied. Chan et al. reported that disease-free was significantly improved in the antiviral treatment group. ${ }^{17}$ The 1-, 3-, and 5-year disease-free survival rates in the treatment group were $66.5 \%, 51.4 \%$, and $51.4 \%$, respectively and those in the control group were $48.9 \%, 33.8 \%$, and $33.8 \%$, respectively ( $\mathrm{P}=0.05$ ). Lamivudine or entecavir were the antiviral agents administered in the 
study, and these drugs significantly reduced early as well as late recurrences. The 1-, 3-, and 5-year overall survival rates in the treatment group were $88.1 \%, 79.1 \%$, and $71.2 \%$, respectively, and those in the control group were $76.5 \%$, $47.5 \%$, and $43.5 \%$, respectively $(\mathrm{P}=0.005)$. Antiviral therapy seemed to prolong the survival by effects of reduction of HCC recurrence and improvement of liver function. ${ }^{17}$ More recently, a large sized cohort study was conducted with 4569 HBV-related HCC patients who received curative resection. There was also significant association between nucleoside analogues (lamivudine, entecavir, or telbivudine) and risk of recurrence (HR 0.68, 95\% CI, 0.53-0.97, p = 0.002) up to 6 years. ${ }^{18}$ Meta-analysis which was previously performed on the efficacy of oral anti-viral therapy in prevention of recurrence after curative treatment of chronic hepatitis B related HCC showed the results consistent with current data. ${ }^{19}$

After liver transplantation, prophylaxis of HBV re-infection with combination of lamivudine and hepatitis $\mathrm{B}$ immune globuine significantly decreases HCC recurrence. In a study with 33.5 months of follow-up, the tumor recurrence free survival was significantly better in patients who received combination therapy and, especially, in those free of viral recurrence. $^{20}$

Therefore, active antiviral treatment is critically needed for the prevention of HCC recurrence after all the curative therapies. In future, efficacy of newer antiviral therapy such as tenofovir need to be evaluated.

\section{2. $\mathrm{HCV}$ - related $\mathrm{HCC}$}

A previous study showed positive relationship between the recurrence of HCC and the serum alanine aminotransferase level in patients with hepatitis C virus (HCV)-associated cirrhosis and HCC after hepatic resection. ${ }^{8}$ This result suggests importance of hepatocytic necrosis in the recurrence of HCC and the significance of decreasing hepatic necroinflammation in the prevention of tumor recurrence. Therefore, antiviral therapy should be needed for these patients. Kubo et al. have demonstrated efficacy of postoperative interferon therapy in decreasing recurrence of HCV-related HCC after resection during median follow-up of 1087 days. The difference seemed greater after 2 years. ${ }^{21}$ Another randomized controlled trial by Shiratori et al. showed that the 2 nd or 3rd recurrence rates are lower in the interferon-treated group than control while the 1st recurrence rates were similar between the groups after ablation therapy. ${ }^{22}$ Long term survival was significantly improved in the interferon-treated group. It is thought that interferon treatment has beneficial effect on prevention of late recurrence, thereby increases survival, especially when achieving sustained virologic response. ${ }^{23}$ This was confirmed by recent meta-analysis (RR 0.33, 95\% CI 0.19-0.58, p < 0.0001). ${ }^{24}$

Pegylated interferon (Peg-interferon) is now widely used for the treatment of chronic hepatitis $\mathrm{C}$ replacing conventional interferon. Combination of peg-interferon and ribavirin increases sustained virologic response rates. Recently, a cohort study reported the efficacy of peg-interferon and ribavirin combination therapy in HCV-related HCC patients after resection. ${ }^{25}$ The recurrence rate of HCC was significantly lower in the 213 treated than 852 untreated patients cohort, with $52.1 \%$ and $63.9 \%$ after 5 years of follow-up, respectively $(\mathrm{P}=0.001)$. The hazard ratio was 0.64 (95\% CI 0.50-0.83), and it thought that type of interferon does not significantly affect the prevention of recurrences either early or late. However, the efficacy of new antiviral therapeutics (directly acting antivirals, DAA) need to be further evaluated in terms of prevention of HCC recurrences.

\section{Other therapies for prevention of late recurrences}

Adjuvant therapies after curative resection has been tired for many years. One study showed efficacy of an acyclic retinoid (polyprenoic acid) in patients with HCC. After a median follow-up of 38 months, 12 patients in the polyprenoic acid group (27 percent) had recurrent or new hepatomas as compared with 22 patients in the placebo group (49 percent, $\mathrm{P}=0.04) .{ }^{26}$ Larger study should be needed.

With the development of molecular biotechnology and tumor immunology, the basic research and clinical application of adoptive immunotherapy have made much progress. ${ }^{27}$ When systemic review was performed, adoptive immunotherapy significantly improve disease-free survival rate or reduced recurrence rate after curative therapy.

Recently a molecular target therapeutic agent, sorafenib, improved survival in Asian patients with advanced HCC. ${ }^{28}$ Efficacy of sorafenib was demonstrated in suppression of postsurgical recurrences and metastasis in an orthotopic mouse model. ${ }^{29}$ Currently a randomized controlled trial for prevention of recurrence after curative therapies is undergoing. (www.clinicaltrial.gov, NCT00692770) The result is anticipated.

\section{Conclusion}

Antiviral therapies significantly reduce the late recurrence rates in patients with hepatitis virus related HCC after curative treatment. Interferon therapy as well as nucleoside analogue therapy are effective for prevention of recurrence of HBV related HCC, and interferon as well as peg-interferon therapy are effective for that of HCV related HCC. Additional studies to confirm the effect of potential adjuvant therapeutic agents are needed. With application of these treatment, the prognosis of HCC will certainly be improved.

\section{References}

1 Imamura H, Matsuyama Y, Tanaka E, Ohkubo T, Hasegawa K, Miyagawa S, Sugawara Y, Minagawa M, Takayama T, Kawasaki S, Makuuchi M. Risk factors contributing to early and late phase intrahepatic recurrence of hepatocellular carcinoma after hepatectomy. J Hepatol 2003;38:200-207.

2 Poon RT, Fan ST, Ng IO, Lo CM, Liu CL, Wong J. Different risk factors and prognosis for early and late intrahepatic recurrence after resection of hepatocellular carcinoma. Cancer 2000;89:500-507.

3 Lee MH, Yang HI, Lu SN, Jen CL, Yeh SH, Liu CJ, Chen PJ, You SL, Wang LY, Chen WJ, Chen CJ. Hepatitis C virus seromarkers 
and subsequent risk of hepatocellular carcinoma: long-term predictors from a community-based cohort study. J Clin Oncol 2010;28:4587-4593.

4 Chen CJ, Yang HI, Su J, Jen CL, You SL, Lu SN, Huang GT, Iloeje UH. Risk of hepatocellular carcinoma across a biological gradient of serum hepatitis B virus DNA level. JAMA 2006;295:65-73.

5 Kubo S, Hirohashi K, Tanaka H, Tsukamoto T, Shuto T, Ikebe T, Yamamoto T, Wakasa K, Nishiguchi S, Kuroki T, Kinoshita $\mathrm{H}$. Risk factors for recurrence after resection of hepatitis $\mathrm{C}$ virus-related hepatocellular carcinoma. World J Surg 2000;24:1559-1565.

6 Kim BK, Park JY, Kim do Y, Kim JK, Kim KS, Choi JS, Moon BS, Han KH, Chon CY, Moon YM, Ahn SH. Persistent hepatitis B viral replication affects recurrence of hepatocellular carcinoma after curative resection. Liver Int 2008;28:393401.

7 Wu JC, Huang YH, Chau GY, Su CW, Lai CR, Lee PC, Huo TI, Sheen IJ, Lee SD, Lui WY. Risk factors for early and late recurrence in hepatitis B-related hepatocellular carcinoma. J Hepatol 2009;51:890-897.

8 Tarao K, Takemiya S, Tamai S, Sugimasa Y, Ohkawa S, Akaike M, Tanabe H, Shimizu A, Yoshida M, Kakita A. Relationship between the recurrence of hepatocellular carcinoma (HCC) and serum alanine aminotransferase levels in hepatectomized patients with hepatitis $C$ virus-associated cirrhosis and HCC. Cancer 1997;79:688-694.

9 Yang XR, Xu Y, Shi GM, Fan J, Zhou J, Ji Y, Sun HC, Qiu SJ, Yu B, Gao Q, He YZ, Qin WZ, Chen RX, Yang GH, Wu B, Lu Q, Wu ZQ, Tang ZY. Cytokeratin 10 and cytokeratin 19: predictive markers for poor prognosis in hepatocellular carcinoma patients after curative resection. Clin Cancer Res 2008;14:3850-3859.

10 Poon RT, Lau C, Pang R, Ng KK, Yuen J, Fan ST. High serum vascular endothelial growth factor levels predict poor prognosis after radiofrequency ablation of hepatocellular carcinoma: importance of tumor biomarker in ablative therapies. Ann Surg Oncol 2007;14:1835-1845.

11 Giordano S, Columbano A. MicroRNAs: new tools for diagnosis, prognosis, and therapy in hepatocellular carcinoma? Hepatology 2013;57:840-847.

12 Sato F, Hatano E, Kitamura K, Myomoto A, Fujiwara T, Takizawa S, Tsuchiya S, Tsujimoto G, Uemoto S, Shimizu K. MicroRNA profile predicts recurrence after resection in patients with hepatocellular carcinoma within the Milan Criteria. PLoS One 2011;6:e16435.

13 Hung IF, Poon RT, Lai CL, Fung J, Fan ST, Yuen MF. Recurrence of hepatitis B-related hepatocellular carcinoma is associated with high viral load at the time of resection. Am J Gastroenterol 2008;103:1663-1673.

14 Someya T, Ikeda K, Saitoh S, Kobayashi M, Hosaka T, Sezaki H, Akuta N, Suzuki F, Suzuki Y, Arase Y, Kumada H. Interferon lowers tumor recurrence rate after surgical resection or ablation of hepatocellular carcinoma: a pilot study of patients with hepatitis B virus-related cirrhosis. J Gastroenterol 2006;41:1206-1213.

15 Sun HC, Tang ZY, Wang L, Qin LX, Ma ZC, Ye QH, Zhang BH, Qian YB, Wu ZQ, Fan J, Zhou XD, Zhou J, Qiu SJ, Shen YF. Postoperative interferon alpha treatment postponed recurrence and improved overall survival in patients after curative resection of HBV-related hepatocellular carcinoma: a randomized clinical trial. J Cancer Res Clin Oncol 2006;132: 458-465.

16 Breitenstein S, Dimitroulis D, Petrowsky H, Puhan MA, Mullhaupt B, Clavien PA. Systematic review and meta-analysis of interferon after curative treatment of hepatocellular carcinoma in patients with viral hepatitis. Br J Surg 2009;96: 975-981.

17 Chan AC, Chok KS, Yuen WK, Chan SC, Poon RT, Lo CM, Fan ST. Impact of antiviral therapy on the survival of patients after major hepatectomy for hepatitis B virus-related hepatocellular carcinoma. Arch Surg 2011;146:675-681.

18 Wu CY, Chen YJ, Ho HJ, Hsu YC, Kuo KN, Wu MS, Lin JT. Association between nucleoside analogues and risk of hepatitis $\mathrm{B}$ virus-related hepatocellular carcinoma recurrence following liver resection. JAMA 2012;308:1906-1914.

19 Wong JS, Wong GL, Tsoi KK, Wong VW, Cheung SY, Chong CN, Wong J, Lee KF, Lai PB, Chan HL. Meta-analysis: the efficacy of anti-viral therapy in prevention of recurrence after curative treatment of chronic hepatitis B-related hepatocellular carcinoma. Aliment Pharmacol Ther 2011;33:1104-1112.

20 Zimmerman MA, Ghobrial RM, Tong MJ, Hiatt JR, Cameron AM, Busuttil RW. Antiviral prophylaxis and recurrence of hepatocellular carcinoma following liver transplantation in patients with hepatitis B. Transplant Proc 2007;39:3276-3280.

21 Kubo S, Nishiguchi S, Hirohashi K, Tanaka H, Shuto T, Yamazaki O, Shiomi S, Tamori A, Oka H, Igawa S, Kuroki T, Kinoshita H. Effects of long-term postoperative interferon-alpha therapy on intrahepatic recurrence after resection of hepatitis $C$ virusrelated hepatocellular carcinoma. A randomized, controlled trial. Ann Intern Med 2001;134:963-967.

22 Shiratori Y, Shiina S, Teratani T, Imamura M, Obi S, Sato S, Koike Y, Yoshida H, Omata M. Interferon therapy after tumor ablation improves prognosis in patients with hepatocellular carcinoma associated with hepatitis $C$ virus. Ann Intern Med 2003;138:299-306.

23 Ishikawa T. Secondary prevention of recurrence by interferon therapy after ablation therapy for hepatocellular carcinoma in chronic hepatitis C patients. World J Gastroenterol 2008;14:6140-6144.

24 Miyake Y, Takaki A, Iwasaki Y, Yamamoto K. Meta-analysis: interferon-alpha prevents the recurrence after curative treatment of hepatitis C virus-related hepatocellular carcinoma. J Viral Hepat 17:287-292.

25 Hsu YC, Ho HJ, Wu MS, Lin JT, Wu CY. Postoperative peginterferon plus ribavirin associated with reduced recurrence of hepatitis $\mathrm{C}$ virus-related hepatocellular carcinoma. Hepatology 2013 in press.

26 Muto Y, Moriwaki H, Ninomiya M, Adachi S, Saito A, Takasaki KT, Tanaka T, Tsurumi K, Okuno M, Tomita E, Nakamura T, Kojima T. Prevention of second primary tumors by an acyclic retinoid, polyprenoic acid, in patients with hepatocellular carcinoma. Hepatoma Prevention Study Group. N Engl J Med 1996;334:1561-1567.

27 Zhong JH, Ma L, Wu LC, Zhao W, Yuan WP, Wu FX, Zhang ZM, Huang S, You XM, Li LQ. Adoptive immunotherapy for postoperative hepatocellular carcinoma: a systematic review. Int J Clin Pract 2012;66:21-27.

28 Cheng AL, Kang YK, Chen Z, Tsao CJ, Qin S, Kim JS, Luo R, Feng J, Ye S, Yang TS, Xu J, Sun Y, Liang H, Liu J, Wang J, Tak WY, Pan H, Burock K, Zou J, Voliotis D, Guan Z. Efficacy and safety of sorafenib in patients in the Asia-Pacific region with advanced hepatocellular carcinoma: a phase III randomised, double-blind, placebo-controlled trial. Lancet Oncol 2009; 10:25-34.

29 Feng YX, Wang T, Deng YZ, Yang P, Li JJ, Guan DX, Yao F, Zhu YQ, Qin Y, Wang H, Li N, Wu MC, Wang HY, Wang XF, Cheng $S Q$, Xie D. Sorafenib suppresses postsurgical recurrence and metastasis of hepatocellular carcinoma in an orthotopic mouse model. Hepatology 2011;53:483-492. 


\section{Curriculum Vitae}

Name

Institution

E-mail
Position
Young Kon Kim

Associate Professor

Department of radiology, Samsung medical center, Sungkyunkwan University school of medicine, Seoul, Korea

jmyr@dreamwiz.com

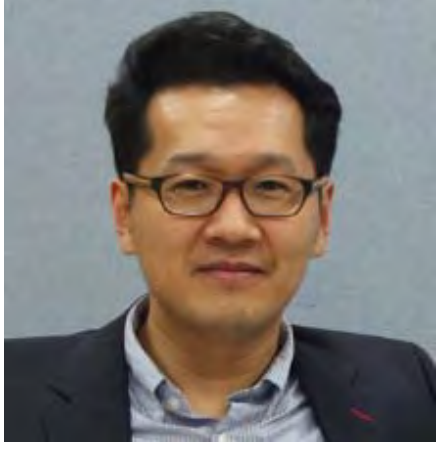

An abdominal radiologist who is enthusiastic in hepato-biliary-pancreas imaging, particularly liver MR imaging. I am always trying to publish articles that provide useful information applicable in daily practice, which in turn help narrow the differential diagnosis as well as increase the diagnostic confidence for focal liver lesions.

\section{Research Interest:}

Abdominal MR imaging

Liver MR Contrast Media

Imaging of focal liver lesion 


\section{Prediction of HCC Recurrence by CT and MRI Young Kon Kim \\ Radiology, Samsung Medical Center, Seoul, Korea}

Since most HCCs develop in patients with chronic liver disease, a surveillance program incorporating imaging modalities has become more important. Non-invasive imaging criteria allowing diagnosis of HCCs based on their vascular profile on CT or MRI have been proposed by study groups. As for HCC recurrence after therapy, local tumor progression or intrahepatic metastasis and multicentric occurrences should be considered. In histology, the local tumor progression or intrahepatic metastasis are differentiated to the same degree or are less differentiated than the main lesion. Thus, clinicians should be sensitive to minimal change in enhancement, morphology of treated area and signal intensity, as well as portal vein thrombosis for early recognition of tumor recurrence. Even though newly developed nodules do not fit HCC imaging criteria such as arterial-only enhancing lesion or hypovascular lesion, tumor recurrence should be considered. MRI has beneficial in the diagnosis of tumor recurrence relative to CT owing to its excellent soft tissue contrast and multiparametric information using $\mathrm{T} 1$ - and $\mathrm{T} 2$-weighted imaging, gadoxetic acid hepatobiliary phase (HBP) and diffusion-weighted imaging (DWI).

With active implementation of a surveillance program for high risk hepatocellular carcinoma patients, early HCC with atypical vascular pattern is being detected with increasing frequency. Using only dynamic imaging with extracellular space contrast agents which rely on highlighting the differ- ences in blood supply, it is difficult to accurately characterize and detect atypical HCC. With the recent introduction of gadoxetic acid (Gd-EOB-DTPA) and DWI in liver MRI, MRI has the potential to improve both the detection and characterization of HCC. In HCC workup, the most useful feature of gadoxetic acid is better depiction of HCC as hypointense on HBP images than on portal phase or equilibrium phase of conventional dynamic CT or MRI. Thus, arterial-only enhancing nodules on dynamic CT or MRI could be accurately characterized by adding HBP image. Sometimes, early HCC can be detected as hypointense only on HBP, but occult on other imaging. Gadoxetic acid HBP image could be also useful in predicting biologic features of HCC. Several reports have shown the iso- or hyperintense HCC on HBP due to contrast uptake tends to be less aggressive (grade I or II) than hypointense HCC on HBP. In addition, microvessel invasion of HCC, an important risk factor predictive of tumor recurrence after resection, could also be predicted with HBP by showing parenchymal hypointensity round tumor. Given that HCC with diffuse fatty change are less aggressive, recognition of intratumoral fat with chemical shift MRI could be useful in predicting tumor aggressiveness. Hypovascular hypointense nodules on gadoxetic acid HBP image are known as high-risk nodules to progress hypervascular HCC. They could be early HCC or dysplastic nodule on histology. Based on our investigation, hyperintensity on DWI is strongly associated with progression of hypovascular hypointense nodules seen on HBP to hypervascular overt HCC. In conclusion, the combination of gadoxetic acid MRI and DWI could be beneficial in the detection and characterization of HCC as well as early recognition of high risk nodule to progress to hyprevascular HCC. 
The 4th Asia-Pacific Primary Liver Cancer Expert Meeting

\section{Curriculum Vitae}

Name

Position

Institution

E-mail
Michiie Sakamoto

Professor

Department of Pathology, Keio University School of Medicine, Tokyo, Japan msakamot@z5.keio.jp

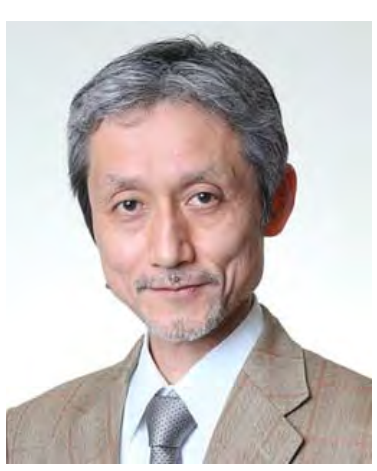

Michiie Sakamoto is currently a Professor at the Department of Pathology, Keio University School of Medicine since 2002. Professor Sakamoto studied Medicine at Keio University and graduated in 1985. Following this, he majored in Pathology at Keio University Graduate School of Medicine and received a PhD degree in 1991. He was appointed as a research member in Pathology Division, National Cancer Center Research Institute in Tokyo in 1989, and nominated as a chief in the division in 1999.

Professor Sakamoto have conducted comprehensive analyses of gene expression correlating with multi-step development and histological subtypes of several types of cancers, and identified molecules applicable to individualized diagnosis and therapy. Especially he has defined early stages of HCC and clarified their molecular features, which contributed to establish the concept of early HCC.

Professor Sakamoto published over 200 international scientific papers in high quality English Journal. He is also serving as an Editor-in-chief of Pathology International, an Associate Editor of Cancer Science and Hepatology Research, and Editorial Board of many kinds of international journals.

\section{Research Interest:}

His research interest is "Molecular Pathology of Cancer: multistep-carcinogenesis, early cancer, invasion and metastasis and Pathology Informatics". 


\section{Prediction by Histology}

\section{Michiie Sakamoto}

Department of Pathology, Keio University School of Medicine, Tokyo, Japan

Prognosis of HCC still remains very poor because of a high incidence of recurrence even after curative treatment. It is widely accepted that poor tumor differentiation and presence of micro vascular invasion are the major histologic risk factors for HCC recurrence. Because HCC nodule is usually consisted with heterogeneous component of tumor differentiation, it should be noted that sampling issue exists in case of biopsy diagnosis of tumor differentiation. Vascular invasion is also evaluable in surgical specimens, but not in biopsy specimens. Histology specimens can be widely used for immunohistochemical analyses, and several immunohistochemical markers are proposed to be useful to predict HCC recurrence. Those markers include tumor suppressor p53, signaling molecules mediating TGF $\beta$ and PI3K signaling pathways, cytoskeletal proteins regulating cell motility, and so-called stem/ progenitor markers. We previously indicated expression of phosphorylated Akt is an independent risk factor for early recurrence after surgery. We also indicated expression of K19 in biopsy specimen is a significant risk factor for recurrence and early recurrence after RFA. In addition to these immunoprofiling approaches, whole slide image analysis combining whole slide image digitization and computational automated image analysis enables complicated morphometrical analysis widely applicable in routine practice. We have just applied this technique to predict HCC recurrence and presence of vascular invasion. These approaches all together will facilitate predictive value of histology for HCC recurrence. 


\section{Curriculum Vitae}

$\begin{array}{ll}\text { Name } & \text { Kelly W. Burak } \\ \text { Position } & \begin{array}{l}\text { Associate Professor of Medicine } \\ \text { Director, University of Calgary }\end{array} \\ & \begin{array}{l}\text { Liver Unit } \\ \text { Institution }\end{array} \\ \text { University of Calgary } \\ \text { E-mail } & \text { kwburak@ucalgary.ca }\end{array}$

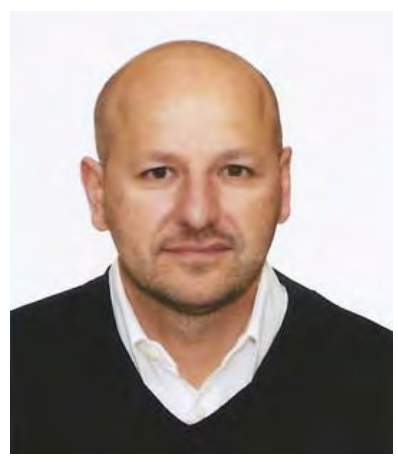

Dr. Kelly Burak completed a BSc (Biology), MD and Internal Medicine training in Saskatchewan, followed by Gastroenterology \& Hepatology training in Calgary and a Liver Transplant fellowship at the Mayo Clinic in Rochester, Minnesota. In 2005, Dr. Burak was awarded his MSc in Epidemiology from the University of Calgary and in 2012 obtained a Graduate Certificate in Clinical Education from Flinders University in Adelaide, Australia. He is an Associate Professor of Medicine and serves as the Director of the Liver Unit and Southern Alberta Liver Transplant Clinic, with a cross appointment in Medical Oncology. His clinical and research interests include liver transplantation and hepatocellular carcinoma. He is an Associate Editor for the Canadian Journal of Gastroenterology. He has won a number of teaching awards, both locally and nationally, including the Canadian Association of Gastroenterology Young Educator Award in 2010 .

\section{Research Interest:}

Clinical and research interests include liver transplantation and hepatocellular carcinoma (HCC).

He has served as the principal investigator on more than 20 clinical trials, including studies involving sorafenib, brivanib and transarterial chemoembolization for HCC. He has been an expert consultant for a health technology assessment of Y90 transarterial radioembolization. He has more than 50 peer reviewed publications, including the Canadian HCC guidelines which he co-authored. 


\section{Prediction of Hepatocellular Carcinoma Recurrence by Molecular Biomarkers}

\author{
Kelly W. Burak
}

Associate Professor of Medicine, Division of Gastroenterology and Hepatology, Departments of Medicine and Oncology, University of Calgary, Calgary, Alberta, Canada

\section{Introduction}

Recurrence following curative therapy for hepatocellular carcinoma (HCC) remains a major problem. Alfa-fetoprotein (AFP), des- $\alpha$-carboxy prothrombin (DCP) and lens culinaris agglutininreactive fraction of AFP (AFP-L3) are biomarkers that have been shown to predict recurrence and overall survival in patients undergoing surgical resection, radiofrequency ablation (RFA) or liver transplantation (LT). Not only can they provide prognosis following curative therapy, but there is emerging evidence that AFP should be used in the selection process for LT. A better understanding of the genetics and molecular pathways involved in HCC will lead to the development of new molecular biomarkers; however, these tests are not yet routinely available.

\section{Recurrence Following Curative Therapy for HCC:}

Recurrence is seen in approximately $70 \%$ of patients within 5 years of surgical resection (1). A recent systematic review of patient undergoing resection within the Milan criteria (single tumour $\leq 5 \mathrm{~cm}$ or three tumours all $\leq 3 \mathrm{~cm}$ ) evaluated 29 studies published in the last decade (2). They demonstrated a median overall survival (OS) at 5 years of $67 \%$ following surgical resection; however, median disease free survival (DFS) at 5 years was only $37 \%$ (2). Early recurrence (within 2 years) after resection is seen in approximately $50 \%$ of patients, and is considered distinct to late recurrence which may relate more to the development of de novo HCC in the setting of cirrhosis (3).

Although overall survival rates may be similar when comparing RFA to surgical resection, recurrence rates following RFA have generally been reported to be higher. A recent comparison of 605 patients undergoing resection or RFA for Barcelona Clinic Liver Cancer (BCLC) stage 0 (very early) or stage A (early) HCC found no difference in $\mathrm{OS}$ after adjusting for important covariates by propensity score, but those undergoing RFA had significantly higher recurrence rates (4). Similarly, three randomized controlled trials from China comparing resection versus RFA for patients with early stage HCC demonstrated lower DFS in patients randomized to receive RFA (5-7), although this difference reached statistical significance in only one trial (6) [see Table 1]. Recurrence following RFA can be categorized as local, which may reflect early treatment failure, or distant which may represent late recurrence with the development of de novo HCC.

Recurrence following LT is typically less than $15 \%$ when patients are selected according to the Milan criteria. Although several groups have reported excellent outcomes with extended criteria for LT, there may be a "price to pay" when the Milan criteria are exceeded (8). A recent meta-analysis of 19 studies found a significant higher overall survival when patient were within Milan criteria [HR 1.68, 95\% CI 1.39-2.03] (9). It is important to note that recurrence following LT is more likely to be at an advanced stage where treatment options are more limited.

\section{Biomarkers for HCC}

Alpha-fetoprotein (AFP) is the most recognized biomarker of primary liver cancer and has been used for the diagnosis of HCC for the past four decades. However, AFP can be falsely elevated by active viral hepatitis and many small HCC do not make AFP, thereby limiting it role as a screening test (1). Ultrasound alone is recommended for routine surveillance, as AFP detects only $7 \%$ of cases not found on screening ultrasound (10). Recently, international practice guidelines have removed AFP from the non-invasive diagnostic algorithms for HCC $(1,10,11)$. However, the prognostic ability of AFP has long been recognized and has led to the incorporation of AFP into many staging systems including GETCH $(\mathrm{AFP}>35)$, CLIP $(\mathrm{AFP}>400)$, and CUPI $(\mathrm{AFP}>500)(12-14)$.

Des- $\alpha$-carboxy prothrombin (DCP), also known as prothrombin induced by vitamin K absence (PIVKA), is an abnormal prothrombin molecule that is produced by HCC by mechanisms that are not completely understood (15). DCP has been shown to be highly specific for the diagnosis of HCC and is associated with prognosis. Lens culinaris agglutinin-reactive fraction of AFP (AFP-L3) is a fucosylated isoform of AFP and is reported as a percentage of total AFP (15). A systematic review of biomarkers suggests that an AFP-L3 cutoff of $15 \%$ is most useful for diagnosing HCC (16). As both DCP and AFP-L3 have been associated with portal vein invasion they are better tests for predicting prognosis rather than for use in surveillance $(17,18)$. Both of these tests are not widely available in Western countries.

Biomarkers to Predict Recurrence After Resection and RFA

AFP, DCP and AFP-L3 have all been shown to predict recurrence following surgical resection. A recent study from China evaluated staging systems and AFP expression in 902 HCC patients undergoing surgical resection (19). The BCLC was the best staging system for AFP positive patients, and elevation of AFP $(>20 \mathrm{ng} / \mathrm{mL})$ was associated with significantly lower survival in BCLC stage 0-A patients (19). Some studies have suggested that preoperative DCP $(>40 \mathrm{mAU} / \mathrm{mL})$ may be superior to AFP $(>20 \mathrm{ng} / \mathrm{mL})$ for predicting recurrence after surgical resection (20). Studies from Japan have shown that elevation of DCP is more likely if patients have vascular invasion, while AFP-L3 is associated with tumour grade (21). Positivity for all three biomarkers (AFP, DCP and AFP-L3) is associated with microvascular invasion (MVI) and more poorly differentiated tumours (22).

AFP has also been shown to predict recurrence after RFA, with a recent study of 330 patients demonstrating a worse 5 year survival in patients with an AFP $\geq 100 \mathrm{ng} / \mathrm{mL}$ (57.4\% versus $75.3 \%$ ) (23). In this experience, low platelets $(<100 \times 109 / \mathrm{L})$, tumour size $(>2 \mathrm{~cm})$ and $<20 \%$ reduction in AFP following RFA predicted recurrent HCC (23). A Japanese study that prospectively followed 227 patients after percutaneous ethanol injection (PEI) or microwave ablation, found DCP to be the strongest predictor of subsequent portal venous invasion (17). Another study evaluating all three biomarkers, determined that AFP $>100 \mathrm{ng} / \mathrm{mL}$ or AFP-L3 $>15 \%$, either before or 2 months after RFA, were the most significant predictors of HCC recurrence (24). However, if the AFP or AFP-L3 fell below these thresholds 2 months after RFA, they were no longer significant predictors of tumour recurrence (24). Similarly, a study of 63 HCC patients undergoing resection found that normalization of DCP, but not changes in AFP, were independently associated with better overall survival (25).

Use of Afp in Selection of Patients for Liver Transplantation

Although DCP has been reported to predict recurrence in Japanese recipients of live donor LT [Kyoto criteria $=\mathrm{HCC}$ size $>5 \mathrm{~cm}$, HCC number $\geq 11$, DCP $>400 \mathrm{mAU} / \mathrm{mL}$ ] (26), most studies have evaluated the impact of waitlist AFP elevations on post transplantation outcomes, using various cut points of $30,100,200,300,400$ or $>1000 \mathrm{ng} / \mathrm{mL}$ (9). A recent study from Canada, suggested that exceeding the Milan criteria or a rising natural AFP slope (NAS) before transplant and were strong predicts of recurrence after LT (27).

Three large studies have evaluated the role of AFP in predicting overall survival (OS) in the United Network for Organ Sharing (UNOS) registry [see Table 2]. Toso and colleagues reported that total tumour volume $(\mathrm{TTV})>115 \mathrm{~cm} 3$ and $\mathrm{AFP}>400 \mathrm{ng} / \mathrm{mL}$ were independent predictors of OS (28). Respecting these criteria led to good results; however, if either criteria were exceeded the 4 year OS was less than $50 \%$ (28). Mailley and colleagues identified 2253 patients in the UNOS registry with AFP values (29). On multivariate analysis, modest 
Table 1. Comparison of disease free survival in three randomized controlled trials of surgical resection versus radiofrequency ablation for early HCC

\begin{tabular}{|c|c|c|c|c|c|c|c|c|}
\hline \multirow[t]{2}{*}{ Author (Reference) } & \multirow[t]{2}{*}{ Inclusion criteria } & \multirow{2}{*}{$\begin{array}{l}\text { Sample Size } \\
(\text { ITT } \rightarrow \text { treated) }\end{array}$} & \multicolumn{5}{|c|}{ Disease Free Survival (\%) } & \multirow{2}{*}{$P$ value } \\
\hline & & & $1 \mathrm{yr}$ & $2 \mathrm{yr}$ & $3 \mathrm{yr}$ & $4 \mathrm{yr}$ & $5 \mathrm{yr}$ & \\
\hline \multirow{2}{*}{$\begin{array}{l}\text { Chen MS } \\
\text { (Ann Surg 2006) }\end{array}$} & \multirow{2}{*}{$\begin{array}{l}1 \mathrm{HCC} \\
\leq 5 \mathrm{~cm}\end{array}$} & Surgery $=90 \rightarrow 109$ & 87 & 77 & 69 & 52 & - & \multirow{2}{*}{ NS } \\
\hline & & $\mathrm{RFA}=90 \rightarrow 71$ & 86 & 69 & 64 & 46 & - & \\
\hline \multirow{2}{*}{$\begin{array}{l}\text { Huang J } \\
\text { (Ann Surg 2010) }\end{array}$} & \multirow[t]{2}{*}{ Milan criteria } & Surgery $=115 \rightarrow 122$ & 85 & 74 & 61 & 55 & 51 & \multirow{2}{*}{0.024} \\
\hline & & $\mathrm{RFA}=115 \rightarrow 108$ & 82 & 59 & 46 & 34 & 27 & \\
\hline \multirow{2}{*}{$\begin{array}{l}\text { Feng K } \\
\text { (J Hepatol 2012) }\end{array}$} & \multirow{2}{*}{$\begin{array}{l}\leq 2 \mathrm{HCC} \\
\leq 4 \mathrm{~cm}\end{array}$} & Surgery $=84$ & 91 & 77 & 61 & - & - & \multirow{2}{*}{0.122} \\
\hline & & $\mathrm{RFA}=84$ & 86 & 67 & 50 & - & - & \\
\hline
\end{tabular}

ITT = intention to treat; HCC = hepatocellular carcinoma; RFA = radiofrequency ablation; yr = year.

Table 2. Three studies of the UNOS registry evaluating the ability of AFP to predict prognosis after LT.

\begin{tabular}{|c|c|c|c|c|}
\hline Author (Reference) & Dates & Sample Size & Categories & $\begin{array}{l}\text { Overall Survival (\%) at } \\
4 \text { years }\end{array}$ \\
\hline \multirow{2}{*}{$\begin{array}{l}\text { Toso C } \\
\text { (Hepatology 2009) }\end{array}$} & \multirow[t]{2}{*}{$2002-2008$} & \multirow{2}{*}{$\begin{array}{l}\mathrm{HCC}=6,478 \\
\mathrm{TTV}>115=18 \\
\mathrm{AFP}>400=465\end{array}$} & $\mathrm{TTV} \leq 115$ AND AFP $\leq 400$ & 60 \\
\hline & & & $\mathrm{TTV}>115$ OR AFP $>400$ & 35 \\
\hline \multirow[t]{3}{*}{$\begin{array}{l}\text { Mailey B } \\
\text { (Arch Surg 2011) }\end{array}$} & \multirow[t]{3}{*}{$2003-2008$} & \multirow[t]{3}{*}{$\mathrm{HCC}=2253$} & $\begin{array}{l}\text { AFP }<20 \\
(n=1210)\end{array}$ & 76 \\
\hline & & & $\begin{array}{l}\text { AFP 20-399 } \\
(\mathrm{n}=805)\end{array}$ & 65 \\
\hline & & & $\begin{array}{l}\mathrm{AFP}>400 \\
(\mathrm{n}=238)\end{array}$ & 57 \\
\hline \multirow[t]{4}{*}{$\begin{array}{l}\text { Berry K } \\
\text { (Liver Transpl 2013) }\end{array}$} & \multirow[t]{4}{*}{$2002-2011$} & \multirow{4}{*}{$\begin{array}{l}\text { No HCC }=31,789 \\
\text { HCC with } \\
\text { eMELD = 10,282 } \\
\text { HCC with } \\
\text { no eMELD = 3,196 }\end{array}$} & $\begin{array}{l}\text { AFP }<15 \\
(\mathrm{n}=4783)\end{array}$ & 78 \\
\hline & & & $\begin{array}{l}\text { AFP 16-65 } \\
(n=1998)\end{array}$ & 70 \\
\hline & & & $\begin{array}{l}\text { AFP 66-320 } \\
(n=1160)\end{array}$ & 65 \\
\hline & & & $\begin{array}{l}\mathrm{AFP}>320 \\
(\mathrm{n}=718)\end{array}$ & 57 \\
\hline
\end{tabular}

AFP = alpha-fetoprotein; eMELD = exemption Model for Endstage Liver Disease points; HCC = hepatocellular carcinoma; TTV = total tumour volume.

elevations of AFP (20-399ng/mL) [HR 1.50, 95\%CI 1.19-1.89] and high AFP (>400ng/mL) [HR 2.11, 95\%CI 1.55-2.88] were independently associated with increased mortality (29). More recently, Berry and Ioannou compared post LT survival in HCC patients, with and without MELD exemption points (eMELD), with non-HCC patients (30). Compared with tumour size or number, post LT survival was more strongly associated with AFP levels (30). Patients with an AFP $<15 \mathrm{ng} / \mathrm{mL}$ had no increased mortality compared to non-HCC recipients, but even modest elevations of AFP $(>66 \mathrm{ng} / \mathrm{mL})$ led to increase mortality in patients transplanted within the Milan criteria [adjusted HR 1.93, 95\%CI 1.74-2.15] (30). However, those who exceed the Milan criteria had no increased mortality if their AFP was $<15 \mathrm{ng} / \mathrm{mL}$ (30). Also, down-staging the AFP level while awaiting LT using locoregional therapy has been associated with better survival following LT $(30,31)$.

This emerging evidence suggests that AFP should be used in liver allocation policy to ensure that LT recipients with HCC have similar outcomes to patients with other indications for transplant. A recent consensus conference on LT allocation for HCC in the USA recommended that eMELD allocation points only be awarded if the AFP is $<500 \mathrm{ng} / \mathrm{mL}$ (32). Some European centres have adopted AFP into their selection criteria based on a French study in which AFP was shown to improve the prognostic ability of the Milan criteria (33). In the French AFP model [see Table 3], $\geq 3$ points was associated with higher rates of recurrence and poorer survival following LT (33). In
Table 3. AFP model for LT proposed by Liver Transplantation French Study Group

\begin{tabular}{llll}
\hline Variable & & Hazard Ratio & Points \\
\hline Size of largest $(\mathrm{cm})$ & $\leq 3$ & 1 & 0 \\
& $3-6$ & 1.31 & 1 \\
& $>6$ & 3.84 & 4 \\
\hline Number & $1-3$ & 1 & 0 \\
& $\geq 4$ & 2.01 & 2 \\
\hline AFP $(\mathrm{ng} / \mathrm{mL})$ & $\leq 100$ & 1 & 0 \\
& $100-1000$ & 1.95 & 2 \\
& $>1000$ & 2.57 & 3 \\
\hline
\end{tabular}

Note: adapted from reference 33 .

Alberta, Canada we have adopted the criteria of TTV $\leq 115 \mathrm{~cm}^{3}$ AND AFP $\leq 400 \mathrm{ng} / \mathrm{mL}$ (34), and this is now being prospectively validated in other centres in Canada and Switzerland.

Other Biomarkers and Future Directions

Other circulating biomarkers that may predict prognosis in HCC include markers of inflammation, including C-reactive protein (CRP) and interleukin-10(IL-10), orother proteins involvedincarcinogenesis 
such as vascular endothelial growth factor (VEGF) and osteopontin (OPN) (36). The molecular markers involved in carcinogenesis of HCC, and how they relate to prognosis, have been summarized elsewhere (37). However, the greatest promise for predicting prognosis after curative therapy is likely to come from molecular and genetic studies of HCC and the surrounding cirrhotic tissue. For example, a 5 -gene score has been recently shown to predict survival following surgical resection and has been validated in HCC patients from Europe, USA and China (38).

\section{Conclusions}

Recurrence following surgical resection and radiofrequency ablation of HCC are very high and serum biomarkers can identify patients at the greatest risk. AFP is the most widely available biomarker and there is emerging evidence that it should be used in the selection of HCC patients for transplantation to further decrease the risk of recurrence.

\section{References}

1 Bruix J, Sherman M. Management of hepatocellular carcinoma: an update. Hepatology 2011;53(3): 1020-1022.

2 Lim KC, Chow PK, Allen JC, et al. Systematic review of outcomes of liver resection for early hepatocellular carcinoma within the Milan criteria. $\mathrm{Br}$ J Surg 2012;99(12): 1622-1629.

3 Cucchetti A, Piscaglia F, Caturelli E, et al. Comparison of recurrence of hepatocellular carcinoma after resection in patients with cirrhosis to its occurrence in a surveilled cirrhotic population. Ann Surg Oncol 2009;16: 413-422.

4 Wang JH, Wang CC, Hung CH, et al. Survival comparison between surgical resection and radiofrequency ablation for patients in BCLC very early/ early stage hepatocellular carcinoma. J Hepatol 2012;56(2): 412-418.

5 Chen MS, Li JQ, Zheng Y, et al. A prospective randomized trial comparing percutaneous local ablative therapy and partial hepatectomy for small hepatocellular carcinoma. Ann Surg 2006;243(30: 321-328.

6 Huang J, Yan L, Cheng Z, et al. A randomized trial comparing radiofrequency ablation and surgical resection for HCC conforming to the Milan criteria. Ann Surg 2010;252(6): 903-912.

7 Feng K, Yan J, Li X, et al. A randomized controlled trial of radiofrequency ablation and surgical resection in the treatment of small hepatocellular carcinoma. J Hepatol 2012;57(4): 794-802.

8 Mazzaferro V. Results of Liver Transplantation: With or Without Milan Criteria? Liver Transpl 2007;13(Suppl 2): S44-S47.

9 Mazzaferro V, Bhoori S, Sposito C, et al. Milan criteria in liver transplantation for hepatocellular carcinoma: an evidence-based analysis of 15 years of experience. Liver Transpl 2011;17(Suppl 2): S44-S57.

10 EASL-EORTC clinical practice guidelines: management of hepatocellular carcinoma. J Hepatol 2012;56(4): 908-943.

11 Omata M, Lesmana LA, Tateishi R, et al. Asian Pacific Association for the Study of the Liver consensus recommendations on hepatocellular carcinoma. Hepatol Int 2010;4(2): 439-474.

12 Chevret S, Trinchet JC, Mathieu D, et al. A new prognostic classification for predicting survival in patients with hepatocellular carcinoma. Groupe d'Etude et de Traitement du Carcinome Hépatocellulaire. J Hepatol 1999;31(1): 133-141.

13 The Cancer of the Liver Italian Program (CLIP) Investigators. Prospective validation of the CLIP score: a new prognostic system for patients with cirrhosis and hepatocellular carcinoma. Hepatology 2000;31(4): 840-845.

14 Leung TW, Tang Am, Zee B, et al. Construction of the Chinese University Prognostic Index for hepatocellular carcinoma and comparison with the TNM staging system, the Okuda staging system, and the Cancer of the Liver Italian Program staging system: a study based on 926 patients. Cancer 2002;94(6): 1760-1769.

15 Lai Q, Melandro F, Pinheiro RS, et al. Alpha-fetoprotein and novel tumor biomarkers as predictors of hepatocellular carcinoma recurrence after surgery: a brilliant star raises again. Int J Hepatol 2012;2012: 893103. Epub 2012 Jun 27.

16 Tateishi R, Yoshida H, Matsuyama Y, Mine N, Kondo Y, Omata M. Diagnostic accuracy of tumor markers for hepatocellular carcinoma: a systematic review. Hepatol Int 2008;2: 17-30.
17 Koike Y, Shiratori Y, Sato S, et al. Des-gammacarboxy prothrombin as a useful predisposing factor for the development of portal venous invasion in patients with hepatocellular carcinoma: a prospective analysis of 227 patients. Cancer 2001;91: 561-569.

18 Carr BI, Kanke F, Wise M, Satomura S. Clinical evaluation of Lens culinaris agglutinin-reactive $\alpha$-fetoprotein and des- $\gamma$-carboxy prothrombin in histologically proven hepatocellular carcinoma in the United States. Dig Dis Sci 2007;52(3): 776-782.

19 Zhou J, Yan T, Bi X, et al. Evaluation of seven different staging systems for alpha-fetoprotein expression in hepatocellular carcinoma after hepatectomy. Tumour Biol 2013;34(2):1061-1070.

20 Kim DY, Paik YH, Ahn SH, et al. PIVKA-II is a useful tumor marker for recurrent hepatocellular carcinoma after surgical resection. Oncology 2007;72(Suppl 1): 52-57.

21 Miyaaki H, Nakashima O, Kurogi M, et al. Lens culinaris agglutininreactive $\alpha$-fetoprotein and protein induced by vitamin K absence II are potential indicators of a poor prognosis: a histopathological study of surgically resected hepatocellular carcinoma. J Gastroenterol 2007;42(12): 962-968.

22 Kiriyama S, Uchiyama K, Ueno M, et al. Triple positive tumor markers for hepatocellular carcinoma are useful predictors of a poor survival. Ann Surg 2011;254: 984-991.

23 Kao WY, Chiou YY, Hung HH, et al. Serum alpha-fetoprotein response can predict prognosis in hepatocellular carcinoma patients undergoing radiofrequency ablation therapy. Clin Radiol 2012;67(5): 429-436.

24 Tateishi R, Shiina S, Yoshida H, et al. Prediction of recurrence of hepatocellular carcinoma after curative ablation using three tumor markers. Hepatology 2006;44(6): 1518-1527.

25 Nanashima A, Sumida Y, Tobinaga S, et al. Postoperative changes in protein-induced vitamin $\mathrm{K}$ absence or antagonist II levels after hepatectomy in patients with hepatocellular carcinoma: relationship to prognosis. HPB (Oxford) 2006;8(2): 137-141.

26 Fujiki M, Takada Y, Ogura Y, et al. Significance of Des-gamma-carboxy prothrombin in selection criteria for living donor liver transplantation for hepatocellular carcinoma. Am J Transpl 2009;9(10): 2362-2371.

27 Dumitra TC, Dumitra S, Metrakos PP, et al. Pretransplantation $\alpha$-fetoprotein slope and Milan criteria: strong predictors of hepatocellular carcinoma recurrence after transplantation. Transplantation 2013;95(1): 228-233.

28 Toso C, Asthana S, Bigam DL. Reassessing selection criteria prior to liver transplantation for hepatocellular carcinoma utilizing the Scientific Registry of Transplant Recipients database. Hepatology 2009;49(3): 832-838.

29 Mailey B, Artinyan A, Khalili J, et al. Evaluation of absolute serum $\alpha$-fetoprotein levels in liver transplant for hepatocellular cancer. Arch Surg 2011;146(1): 26-33.

30 Berry K, Ioannou GN. Serum alpha-fetoprotein level independently predicts post transplant survival in patients with hepatocellular carcinoma. Liver Transpl 2013;19(6): 634-645.

31 Merani S, Majno P, Kneteman NM, et al. The impact of waiting list alphafetoprotein changes on the outcome of liver transplant for hepatocellular carcinoma. J Hepatol 2011;55(4): 814-819.

32 Pomfret EA, Washburn K, Wald C, et al. Report of a national conference on liver allocation in patients with hepatocellular carcinoma in the United States. Liver Transpl 2010;6(3): 262-278.

33 Duvoux C, Roudot-Thoraval F, Decaens T, et al. Liver transplantation for hepatocellular carcinoma: a model including alpha-fetoprotein improves the performance of Milan criteria. Gastroenterology 2012;143(4): 986-994.

34 Burak KW, Kneteman NM. An evidence-based multidisciplinary approach to the management of hepatocellular carcinoma (HCC): The Alberta HCC algorithm. Can J Gastroenterol 2010;24(11): 643-650.

35 Wong KF, Xu Z, Chen J, et al. Circulating markers for prognosis of hepatocellular carcinoma. Exp Opin Med Diagn 2013 May 6 [Epub ahead of print].

36 Mann CD, Neal CP, Garcea G, et al. Prognostic molecular markers in hepatocellular carcinoma: a systematic review. Eur J Cancer 2007;43(6): 979-992.

37 Nault JC, De Reynies A, Villanueva A, et al. A hepatocellular carcinoma 5-gene score associated with survival of patients after liver resection. Gastroenterology 2013 Apr 6 [Epub ahead of print]. 


\section{Curriculum Vitae}

$\begin{array}{ll}\text { Name } & \text { Morris Sherman } \\ \text { Position } & \text { Associate Professor } \\ \text { Institution } & \text { University of Toronto } \\ \text { E-mail } & \text { morris.sherman@uhn.on.ca }\end{array}$

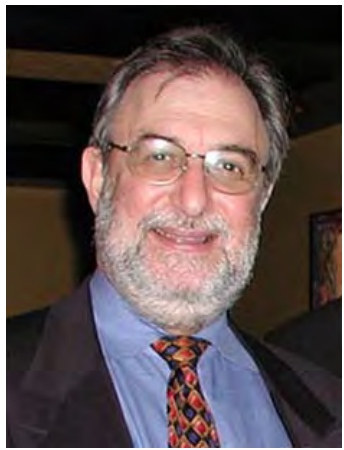

Education:

1978 May-1981 Apr PhD, Protein Biochemistry, Dept of Medicine, University of Cape Town, Cape Town, Western Cape, South Africa, Supervisor(s): Ralph Kirsch

1967 Jul - 1972 Jun MB BCh, Medicine, University of Witwatersrand, Johannesburg, Gauteng, South Africa

Postgraduate, Research and Specialty Training:

1982 Jul-1984 Apr Molecular biology, Liver Research Center, Albert Einstein College of Medicine, New York, New York, United States, Supervisor(s): Dr David Shafritz

1978 Apr - 1981 Apr Protein Biochemistry, Dept of Medicine, University of Cape Town, Supervisor(s): Professor Ralph Kirsch

Qualifications, Certifications and Licenses:

1985 Sep-2011 Mar Fellow of the Royal College of Medicine of Canada, Internal Medicine, Royal College of Medicine of Canada, Ontario, Canada, 275304

1984 Sep - 2011 Feb Licensee, Internal Medicine, College of Physicians and Surgeons of Ontario, Ontario, Canada, 54922

\section{Most Significant Publications:}

1 Bruix J, Sherman M; Practice Guidelines Committee, American Association for the Study of Liver Diseases. Management of hepatocellular carcinoma, an update. Hepatology.2011 Mar;53(3):10201022. Co-Principal Author.

This is an update of the 2005 guidelines and has been cited 379 times.

2 Llovet JM, Di Bisceglie AM, Bruix J, Kramer BS, Lencioni R, Zhu AX, Sherman M, Schwartz M, Lotze M, Talwalkar J, Gores GJ; Panel of Experts in HCC-Design Clinical Trials. Design and endpoints of clinical trials in hepatocellular carcinoma. J Natl Cancer Inst. 2008 May 21;100(10):698-711. Coauthor or Collaborator.

This panel established guidelines for testing of new drugs for treatment of hepatocellular carcinoma. The article has been cited 525 times.

3 Bruix J, Sherman M; Practice Guidelines Committee, American Association for the Study of Liver Diseases. Management of hepatocellular carcinoma. Hepatology. 2005 Nov;42(5):1208-1236. Co-Principal Author.

This was the first North American practice guideline for the management of hepatocellular carcinoma. It was widely adopted. The impact of this article can be gauged by the fact that it has been cited 2095 times.

4 Bruix J, Sherman M, Llovet JM, Beaugrand M, Lencioni R, Burroughs AK, Christensen E, Pagliaro L, Colombo M, Rodés J; EASL Panel of Experts on HCC. Clinical management of hepatocellular carcinoma. Conclusions of the Barcelona-2000 EASL conference. J Hepatol. 2001 Sep;35(3):421430. Co-Principal Author.

This was the first international guideline on the management of hepatocellular carcinoma. It has been cited 1773 times.

5 Schalm SW, Heathcote J, Cianciara J, Farrell G, Sherman M, Willems B, Dhillon A, Moorat A, Barber J, Gray DF. Lamivudine and alpha interferon combination treatment of patients with chronic hepatitis B infection: a randomised trial. Gut. 2000 Apr;46(4):562-568. Coauthor or Collaborator.

This was the publication arising out of the phase 3 trials of lamivudine in hepatitis B. This was the first report of viral suppression in this disease using direct acting nucleoside analogues, and led to the introduction of newer direct acting agents that are now standard therapy. 


\section{Cost Efficacy of Screening for Hepatocellular Carcinoma}

\section{Morris Sherman}

University of Toronto, Toronto, Canada

Cost efficacy analysis has been used to determine whether it is overall beneficial for patients at risk to be screened for hepatocellular carcinoma (HCC). In addition these analyses have been used to determine the threshold level of risk at which screening becomes cost effective, and below which screening is not cost effective. Almost all analyses are sensitive to the incidence of HCC, and most are sensitive to the cost of screening. Most analyses find that screening with ultrasound with or without additional alphafetoprotein (AFP) testing is cost effective. However, the results of analyses vary, with some suggesting that AFP brings additional benefit and some suggesting that it does not. Most analyses show that using CT or MRI for HCC screening is not cost effective.

The incidence of HCC at which screening in patients with chronic hepatitis B is cost effective as at about $0.2 \%$ /year, whereas in other forms of cirrhosis the incidence threshold for which screening becomes cost effective is 1.5-2\%/year.

We have conducted a systematic review and metaanalysis of studies of cost efficacy in HCC screening. Ten studies were of suitable quality. This analysis has shown that screening with ultrasound alone at 6 monthly intervals is the most cost effective form of screening. This holds true over a wide range of gross domestic product. 


\section{Curriculum Vitae}

Name

Position

Institution

E-mail
Ryosuke Tateishi

Assistant Professor

Department of Gastroenterology The University of Tokyo Graduate School of Medicine

tateishi-tky@umin.ac.jp

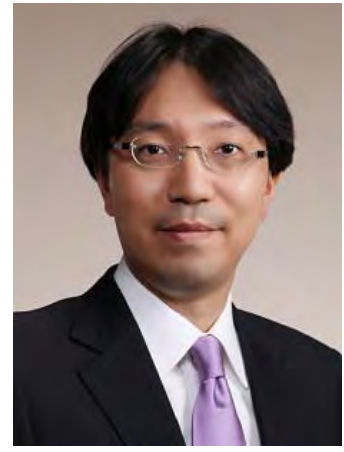

Dr. Ryosuke Tateishi has been working on hepatocellular carcinoma (HCC) since 1998 when he started his PhD in Hepatology at the University of Tokyo, Japan where he graduated in 2002. During his PhD, he studied the prognostic factors of patients with HCC and developed a new scoring system named Tokyo Score. Since 2006, he has been Assistant Professor at the Department of Gastroenterology, Graduate School of Medicine, The University of Tokyo where he continues working on diagnosis and treatment of HCC, particularly on those who underwent percutaneous treatment. He is currently a member of Committee for the General Rules for the Clinical and Pathological Study of Primary Liver Cancer in Liver Cancer Study Group of Japan and Working Committee for Japanese Clinical Practice Guideline for Liver Cancer. Dr. Tateishi published 100 international scientific papers in high quality English Journal including Gastroenterology, Hepatology, Gut and Journal of Hepatology.

\section{Research Interest:}

Epidemiology, surveillance, diagnosis, and treatment of hepatocellular carcinoma

Viral hepatitis

Non-alcoholic fatty liver disease 


\section{Role of Tumor Markers: Beyond Sensitivity and Specificity}

Ryosuke Tateishi

Department of Gastroenterology, Graduate School of Medicine, The University of Tokyo, Tokyo, Japan

The role of tumor markers in surveillance for HCC is still controversial whereas ultrasonography is considered as indispensable. In general, the diagnostic ability of tumor markers has been evaluated with sensitivity, specificity, and receiver operating characteristics (ROC) curve. In such a situation, the diagnostic accuracy is discussed with a single cut-off value. AFP has been criticized because it lacks specificity and more specific markers like des-gamma-carboxyprothrombin (DCP) or Lens culinaris agglutinin-reactive fraction of AFP (AFP-L3) have been reprehended for their suboptimal sensitivities. Since there is an inverse association between sensitivity and specificity that differs according to cut-off values, investigators have been struggling to improve the sensitivity or specificity of a tumor maker by increasing or decreasing its cut-cut off value. However we clinicians know that all positive results are the same. When a cut-off value of $20 \mathrm{ng} / \mathrm{mL}$ is set for AFP, a value of $30 \mathrm{ng} / \mathrm{ml}$ is not predictive of having HCC, whereas $1000 \mathrm{ng} / \mathrm{mL}$ is almost definitive. Stratum-specific likelihood ratio (SSLR) is a more informative method to interpret diagnostic information than sensitivity and specificity with single cut-off value. In SSLR approach, likelihood ratios are calculated with multiple break-points like 20,100, 200 and $400 \mathrm{ng} / \mathrm{mL}$ for AFP. Bayes' theorem is a theory according to which post-test probability is calculated from pre-test probability and likelihood ratios. Bayes' theorem is now utilized universally in various automated decision process like spam-filter as well as clinical decision making. Although ultrasonography plays a major role in the surveillance for HCC, its accuracy is significantly affected by the various factors including operator's skill, performance of the equipment, liver parenchymal atrophy and coarseness and patient's obesity. Therefore the likelihood ratios for positive or negative results in ultrasound examination should be different according to patient's characteristics. We have developed a score, which consists of 3 items (atrophy, obesity and parenchymal texture) to evaluate the reliability of ultrasound examination. By using diagnostic algorithm including SSLRs of tumor markers and ultrasonography reliability score, clinicians would be able to follow the diagnostic process in the surveillance for HCC in a more efficient and sophisticated manner. 


\section{Curriculum Vitae}

$\begin{array}{ll}\text { Name } & \text { Jeong Min Lee } \\ \text { Position } & \text { Radiology Professor } \\ \text { Institution } & \text { Seoul National University Hospital } \\ \text { E-mail } & \text { jmsh@snu.ac.kr }\end{array}$

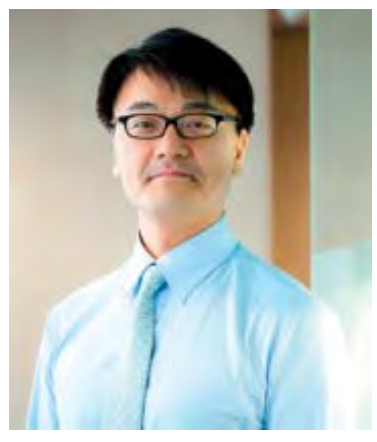

\section{Education:}

College of Medicine - M.D. Cheonbuk National University (Mar. 1984 - Feb. 1990) Graduate School - M.S. Cheonbuk National University (Mar. 1991 - Aug. 1993)

Graduate School - Ph.D. Chungnam National University (Mar. 2001 - Feb. 2004)

\section{Academic Appointments:}

Instructor of Radiology: Cheonbuk National University, College of Medicine (Mar. 2000-Feb. 2002)

Assistant Professor of Radiology: Cheonbuk National University, College of Medicine (Mar. 20002-0ct. 2002)

Assistant Professor of Radiology: Seoul National University, College of Medicine (Nov. 2002-Feb.2007)

Visiting Professor of Radiology (Attending Physician): Iowa University Hospitals \& Clinics (Jul. 2007-Jun. 2008)

Professor of Radiology: Seoul National University, College of Medicine (Mar. 2012-)

\section{Administrative Responsibilities \& Academic Committees:}

Scientific Committee Chair, Korean Radiological Society

Liaison Committee Chair, Korean Abdominal Radiological Society

Education Committee Chair, Korean Society of Magnetic Resonance Imaging in Medicine (2010)

Liaison Committee Chair, The Korean Association for the Study of the Liver Manuscript Reviewer of Radiology, AJR, Investigative Radiology, and KJR Editorial Board Member of Journal of Korean Society of Ultrasound in Medicine and Korean Journal of Radiology

\section{Research Interest:}

Hepatobiliary pancreas imaging, and image guided tumor ablation 


\section{Diagnosis of Very Early Stage of HCC}

\section{Jeong Min Lee}

Seoul National University Hospital, Seoul, Korea

Several previous studies demonstrated that the key to long-term outcome in patients with liver cirrhosis and hepatocellular carcinoma (HCC) is early diagnosis and definitive treatment of HCC. For instance, curative treatment options such as surgical resection, liver transplantation, or radiofrequency ablation can be applied for very early stage or early stage HCCs in cirrhotic patients. Many studies have demonstrated that those curative treatments for very early stage or early stage HCCs could provide at least 50- 70\% 5 year survival rates, which is much higher than intermediate stage HCC. Therefore, there have been increasing evidence for necessity of sensitive imaging modality for detection of small HCC. For the non-invasive diagnosis of HCC, the European Association for the Study of the Liver (EASL) and the American Association for the Study of Liver Diseases (AASLD) emphasize the importance of dynamic-phase imaging using magnetic resonance imaging (MRI) and CT to identify hypervascularity. However, approximately $30 \%$ of pathology confirmed HCCs can show atypical enhancement pattern on dynamic CT or MRI. Recent data suggest that the mean sensitivity of hepatobiliary contrast agent enhanced MRI is significantly superior to 64-slice CT for the detection of HCC. In addition, the wash-in and wash-out observed with Gd-EOB-DTPA are highly reproducible and provide superior images to multidetector CT. Furthermore, although further data on use of Gd-EOB-DTPA in grading cirrhotic lesions, as well as studies correlating MRI and pathological findings, are required, Gd-EOB-DTPA seems to allow the detection of early pathological changes, thus extending the options for the management of cirrhotic patients. Therefore, I believe that as the utility of liver-specific (MRI) increases, it is pertinent to optimize and expand protocols to improve accuracy and foster evolution of techniques; in turn, positive impacts should be seen in patient management. 


\section{Curriculum Vitae}

$\begin{array}{ll}\text { Name } & \text { Do Young Kim } \\ \text { Position } & \begin{array}{l}\text { Associate professor } \\ \text { Institution }\end{array} \\ & \begin{array}{l}\text { Department of Internal Medicine, } \\ \text { Yonsei University College of } \\ \text { Medicine }\end{array} \\ \text { E-mail } & \text { dyk1025@yuhs.ac }\end{array}$

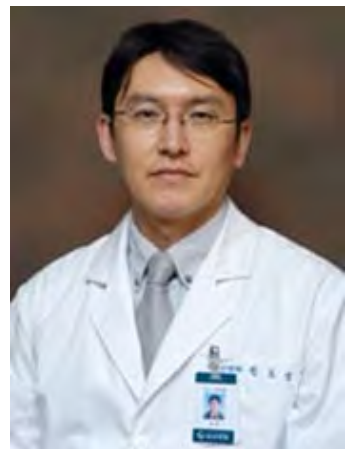

Do Young Kim is now an associate professor of Internal Medicine at Yonsei University College of Medicine, Seoul, Korea, and a hepatologist in the Severance Hospital where he has been a faculty member since 2007. He graduated Yonsei University at 1996, and completed training course in Severance Hospital from 1996 to 2001 . He studied proteomics and microRNA in hepatocellular carcinoma (HCC) at Fred Hutchinson Cancer Research Center as a research associate between 2011 and 2012 .

He has been a vice secretary general in Korean Association of Gastroenterology from 2007 to 2009, and in Korean Liver Cancer Study Group between 2009 and 2010. He is also a member of Korean Association for the Study of Liver Disease (KASL) and International Liver Cancer Association (ILCA).

His main research interest is biomarker for HCC and treatment of advanced HCC. He is one of the investigators in several clinical trials related to molecular target drugs for HCC. He published approximately 100 international original articles, and is a reviewer of Liver International, Gut and Liver, Molecular and Clinical Hepatology, and Journal of Korean Medical Sciences. He is also a editorial board member of World Journal of Hepatology and Yonsei Medical Journal.

\section{Research Interest:}

Biomarker discovery for early HCC diagnosis

MicroRNA

Molecular target therapy 


\section{Monitoring after Curative Treatment: Role of Tumor Markers}

\section{Do Young Kim}

Department of Internal Medicine, Yonsei University College of Medicine, Seoul, Korea

Biomarkers such as alpha-fetoprotein (AFP), des-gammacarboxyprothrombin (DCP) have several roles in the surveillance, diagnosis, monitoring, and prognosis prediction of hepatocellular carcinoma (HCC). Although Western point of view does not agree to use biomarkers in surveillance and diagnosis of HCC, both Eastern and Western physicians seem to utilize these simple serum markers for monitoring after treatment.

Surgical resection as a curative therapy for HCC implies that all malignant cells have been eradicated, which in turn means that tumor markers should be normalized. Accordingly, tumor marker concentration in serum few months after resection is important to assess whether any malignant focus remains or not. For example, decreasing levels of tumor markers, but still high, when measured 1 or 2 months after resection might predict intrahepatic or extrahepatic early recurrence of HCC. What has to be highlighted is that imaging modality such as computed tomography (CT) scan of liver does not show recurrent HCC lesions at early post-operative period.

Since pre-operative high levels of tumor markers are related with higher rate of recurrence and worse outcome compared with low levels of tumor markers, it is necessary to carefully monitor the tumor markers especially. The clonal change at the time of recurrence may result in different pattern of tumor marker elevation. A patient can have normal AFP levels but high DCP levels at recurrence even though he had opposite pattern of tumor marker levels at the first diagnosis of HCC. Thus, monitoring of recurrence with two complementary biomarkers might be helpful.

Monitoring of recurrence just by imaging can miss extrahepatic areas such as upper lung, pelvis, spine. Although the sensitivity of biomarkers in detecting extrahepatic small recurrent lesion is not high, measurement of biomarkers in monitoring is complement to imaging modality.

Lastly, the role of new biomarker, for example highly sensitive AFP-L3, needs to be validated in monitoring of recurrence after curative treatment of HCC. 


\section{Curriculum Vitae}

$\begin{array}{ll}\text { Name } & \text { Riccardo Lencioni } \\ \text { Position } & \begin{array}{l}\text { Professor and Director, Diagnostic } \\ \text { Imaging and Intervention }\end{array} \\ \text { Institution } & \begin{array}{l}\text { University of Pisa, School of } \\ \text { Medicine, Pisa, Italy }\end{array} \\ \text { E-mail } & \begin{array}{l}\text { riccardo.lencioni@med.unipi.it }\end{array}\end{array}$

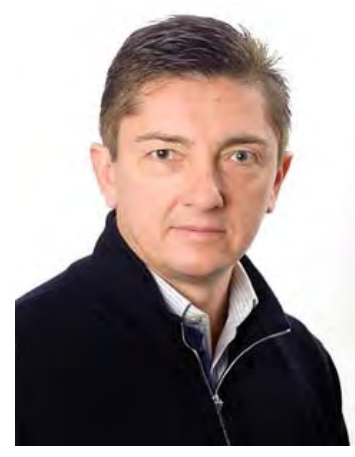

Riccardo Lencioni, MD, FSIR, EBIR, is Professor and Director of the Division of Diagnostic Imaging and Intervention at Pisa University School of Medicine in Pisa, Italy. Riccardo Lencioni has been a leading member of several expert panels developing recommendations for research and clinical management of hepatocellular carcinoma. He has co-authored the white papers Design and Endpoints in Clinical Trials in Hepatocellular Carcinoma (2008), Modified RECIST (mRECIST) Assessment for Hepatocellular Carcinoma (2010), and EASL-EORTC Clinical Practice Guidelines: Management of Hepatocellular Carcinoma (2012).

Riccardo Lencioni is the Chairman of the Board of Directors of the World Conference on Interventional Oncology. He is a co-Founder of the International Liver Cancer Association, in which he also acts as the Executive Secretary. Riccardo Lencioni has authored 170 articles in peer-reviewed international journals, has written 61 textbook chapters, and has been the editor of seven books. Citations of his publications currently number in excess of 9,000. He has been an invited or honorary lecturer at more than 300 international meetings or conferences.

\section{Research Interest:}

Interventional treatment of HCC, combination of loco-regional and systemic treatment of HCC, imaging assessment of HCC response to therapy. 


\section{Imaging Assessment of Tumor Response}

\section{Riccardo Lencioni}

University of Pisa School of Medicine, Pisa, Italy

Tumor response in oncology is usually measured according to Response Evaluation Criteria in Solid Tumors (RECIST).TheRECISTguidelinedefinesstandardmeasurement methods for converting radiology image observations into a quantitative and statistically tractable framework for measuring the response of tumor size to therapy. The RECIST model - developed in 2000 and revised in 2009 with the publication of the version 1.1 - was designed for evaluation of cytotoxic agents. It offers a simple approach to determining anatomic size and lesion changes during treatment as an indicator of response. However, it does not address measures of antitumor activity other than tumor shrinkage and was shown to be unable to capture the anticancer activity of tumordirected therapies used in the treatment of HCC, such as radio- frequency ablation (RFA) and transarterial chemoembolization (TACE). Given the poor correlation observed between response evaluation by conventional metrics and overall survival, a group of experts in HCC clinical trials convened by the American Association for the Study of Liver introduced the concept of viable tumor in HCC response assessment, and proposed specific amendments to standard RECIST. The resulting criteria were named modified RECIST (mRECIST) for HCC. The mRECIST criteria have been endorsed by several scientific societies and organizations and have been increasingly used in HCC clinical research. A growing body of scientific evidence suggests that mRECIST- designed for response assessment in clinical trials - may translate into a tool for clinical practice, a process that has already started with the recommendations issued in the recent clinical practice guidelines produced by the European Association for Study of Liver (EASL) and the European Organization for Research and Treatment of Cancer and by the European Society of Medical Oncology and the European Society of Digestive Oncology. 


\section{Curriculum Vitae}

$\begin{array}{ll}\text { Name } & \text { Norihiro Kokudo } \\ \text { Position } & \text { Professor and Chairman } \\ \text { Institution } & \begin{array}{l}\text { Division of HPB Surgery, } \\ \text { Department of Surgery } \\ \end{array} \\ & \text { The University of Tokyo } \\ \text { E-mail } & \text { KOKUDO-2SU@h.u-tokyo.ac.jp }\end{array}$

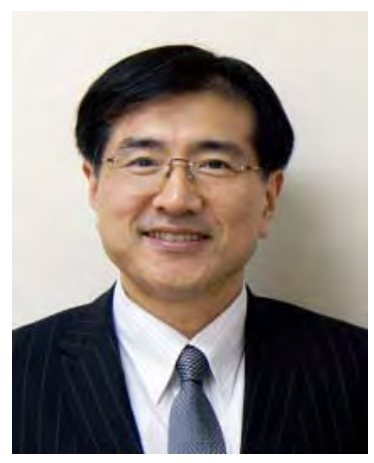

Dr. Norihiro Kokudo is the professor and chairman at Hepato-BiliaryPancreatic Surgery Division and Artificial Organ and Transplantation Division, Department of Surgery, University of Tokyo Hospital. He earned his M.D. in 1981, and then Ph.D. in 1988 at University of Tokyo. From 1989 to 1991 he stayed at Department of Surgery, University of Michigan as a visiting research investigator. After 6 years at Cancer Institute Hospital, Tokyo, as a senior staff of GI surgery, he joined the current institution as an associate professor in 2001 . He then rose to the current position in 2007. Dr. Kokudo has been conducting a number of research projects on surgical treatment of HCC, colorectal liver metastases, and living donor liver transplantation. As a vice-general administrator, he compiled $2^{\text {nd }}$ version of Japanese clinical practice guidelines for HCC in 2009 (Hepatol Res 2010; 40 suppl). He is currently compiling the $3^{\text {rd }}$ version as the chairman of the guideline committee. In April 2012, Dr. Kokudo was appointed as the President of Japan Surgical Society. He is a secretary elect for Asian-Pacific Hepato-Pancreato-Biliary Association (A-PHPBA) and a member-at-large of International Hepato-Pancreato-Biliary Association (IHPBA). He is also a member of International Society of Surgery and The Society of Surgical Oncology. He is an associate editor of Hepatology Research, Liver Cancer, and Surgery Today, and on the editorial board of World Journal of Surgery, Journal of HPB Science, HPB, Japanese Journal of Clinical Oncology, and Hepatogastroenterology.

Research Interest:

Hepatolcellular carcinoma, Colorectal liver metastases, liver transplantation. 


\section{Multiple Repeat Hepatectomy Is a Feasible and Effective Treatment for Recurrent Hepatocellular Carcinoma}

Norihiro Kokudo, Yoshihiro Mise, Yasuhiko Sugawara, and Kiyoshi Hasegawa

Hepato-Biliary-Pancreatic Surgery Division, Department of Surgery, Graduate School of Medicine, University of Tokyo, Tokyo, Japan

Background: The second hepatectomy has been accepted as an effective treatment for intrahepatic HCC recurrence after the primary resection, providing comparable long-term survivals with those of initial hepatectomy. However, the feasibility and prognostic benefits of multiple repeat resection including 3 or more times have not been adequately assessed.

Patients and Methods: From 1994 to 2012, 1341 patients underwent curative hepatectomy for HCC in The University of Tokyo Hospital. Among them, 941 underwent the 1st hepatectomy for the primary HCC and 289 underwent the 2 nd hepatectomy for the 1 st recurrence of HCC. The remaining 110 patients underwent multiple repeat hepatectomy (3rd: 74, 4th: 28, and 5th: 8). Patient demographics, tumor characteristics, surgical outcomes, and long-term survivals were compared among those with initial hepa- tectomy (1st Hx), those with 2nd hepatectomy (2nd Hx), and those with 3 or more hepatectomies (multi Hx).

Results: No significant differences were found in ChildPugh grades or ICGR15 values among the 3 groups. Multiple tumors were more frequently resected in 2nd $\mathrm{Hx}(41 \%)$ and multi $\mathrm{Hx}(42 \%)$, compared with 1 st Hx $(32 \%, \mathrm{p}<0.01)$. Tumor size was significantly larger in 1 st $\mathrm{Hx}$ (median: $3.5 \mathrm{~cm}$, range:0.7-33 cm) than in 2nd $\mathrm{Hx}$ (median: 1.8, range: $0.6-9.0 \mathrm{~cm}$ ) and multi $\mathrm{Hx}$ (median: 1.8 , range: $0.6-6.1 \mathrm{~cm}, \mathrm{p}<$ $0.01)$. Overall morbidity rates were not different among the 3 groups. However, Bile leakage and would infection were more frequently observed in multi $\mathrm{Hx}(12.5 \%, 2.9 \%)$ than in $2 \mathrm{nd} \mathrm{Hx}$ (6.2\% [p $=0.04], 0.4 \%[\mathrm{p}=0.03]$ ). 3/5-year disease free survival rates were $36.6 \% / 27.1 \%$ in 1 st $\mathrm{Hx}, 24.4 \% / 17.9 \%$ in 2nd Hx (p < 0.001 [1st vs. 2nd]), and 21.0\%/12.8\% in multi Hx ( $p<0.01$ [1st vs. multi], $p=0.95$ [2nd vs. multi]). 3/5-year overall survival rates were not significantly different among the 3 groups (1st Hx: 79.4\%/65.3\%, 2nd Hx: 76.7\%/60.5\%, multi Hx: 81.5\%/68.2\%).

Conclusions: The technical complexity of multiple repeat hepatectomy is associated with the increased risks of postoperative bile leakage and wound infection. However, multiple repeat hepatectomy offers comparable disease-free and overall survivals to the second hepatectomy. 


\section{Curriculum Vitae}

$\begin{array}{ll}\text { Name } & \text { Guohong Han } \\ \text { Position } & \text { Professor, Chief } \\ \text { Institution } & \text { Department of Digestive } \\ & \text { Interventional Radiology, Xijing } \\ & \text { Hospital, Fourth Military Medical } \\ & \text { University } \\ \text { E-mail } & \text { hangh@fmmu.edu.cn }\end{array}$

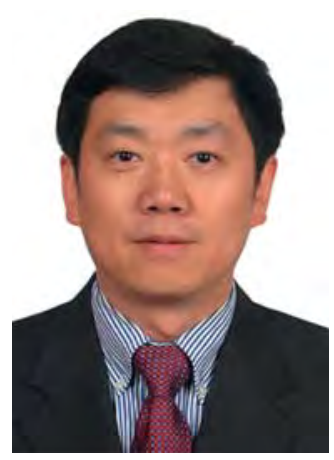

Guohong Han is Professor and Chief of the Department of Digestive Interventional Radiology, Xijing Hospital, Fourth Military Medical University, Xi'an, China, and he complete-d his medical training and PhD with the Department of Radiology at this hospital. Professor Han has also trained in radiology at the department of radiology, School of Medicine, Kanazawa University, Japan, and at the department of Radiology, Queen Mary Hospital, University of Hong Kong.

Professor Han's research interests include developing treatments for hepatocellular carcinoma (HCC) pharmacologically and by transarterial chemoembolization (TACE). He has participated in more than 10 clinical trials involving both HCC treatment and TACE. Professor Han has publicshed approximately 50 journal articles between 2010 and 2013 in Hepatology, Journal of Hepatology, Radiology, Annals of Oncology, Thrombosis and Haemostasis etc., with the most recent three publications studying the use of sorafenib in combination with TACE for the treatment of HCC. 


\section{Management of HCC Recurrence after Curative Treatment Debate: Non-Surgical Approach}

\author{
Guohong Han, Yan Zhao
}

Department of Liver Disease and Digestive Interventional Radiology, Xijing Hospital of Digestive Diseases, Fourth Military Medical University, Xi'an, China

Tumor recurrence represents the major complication of curative therapy. The management of HCC recurrence after curative therapies is a very difficult challenge. There are different treatment methods for the recurrent hepatocellular carcinoma. However, no consensus has been reached so far.

Local ablative techniques, such as percutaneous ethanol injection (PEI), microwave (MW) therapy, and radiofrequency ablation (RFA) have become increasingly popular in the treatment of small recurrent HCC. Among these techniques, RFA is currently the most widely used method due to its ease of use, safety, cost-effectiveness, and minimal invasiveness. It was reported that the treatment with RFA for recurrent HCC was a significant favorable prognostic factor after intrahepatic distant recurrence [1]. Child-Pugh A patients with nonsurgical small tumors seems the ideal candidates to RFA. It is reasonable to perform the combination of RFA and PEI in patients with larger tumors $(3-5 \mathrm{~cm})$, multiple tumors $(3$ nodules $<3 \mathrm{~cm}$ ) and advanced liver failure (Child-Pugh B) [2]. Microwave ablation has its special features, such as consistently higher intratumoral temperatures, larger tumor ablation volumes, faster ablation times and an improved convection profile [3]. There was one randomized controlled trial (RCT) to compare the effectiveness of MW against that of RFA; however, no statistically significant differences were found.

Treatment of unresectable HCC by transarterial chemoembolization (TACE) has been reported to have satisfactory results [4-5]. However, it should be noted that the patients should have reserved liver function (mostly Child-Pugh A or B7) because the ischemic insult can lead to severe adverse events. It was suggested that tumor size $>2 \mathrm{~cm}$ has been reported to be a risk factor for local tumor progression after RFA in patients with small HCC. Thus, the TACE combined with RFA was believed to reduce the local progression rate and improve the overall survival [6-7].

Well-designed RCTs are urgently needed to help establish the optimum treatment algorithm for patients with unresectable HCC whose disease recurs after initial curative therapy.

\section{References}

1 Okuwaki Y, Nakazawa T, Kokubu S, et al. Repeat radiofrequency ablation provides survival benefit in patients with intrahepatic distant recurrence of hepatocellular carcinoma. Am J Gastroenterol 2009;104:2747-2753.

2 European Association for the study of the liver. EASL-EORTC clinical practice guidelines: management of hepatocellular carcinoma. J Hepatol 2012;56:908-943.

3 Lencioni R, Crocetti L. Local-regional treatment of hepatocellular carcinoma. Radiology 2012;262:43-58.

4 Lo CM, Ngan H, Tso WK, et al. randomized controlled trial of transarterial lipiodol chemoembolization for unresectable hepatocellular carcinoma. Hepatology 2002;35:1164-1171.

5 Llovet JM, Real MI, Montana X, et al. Arterial embolization or chemoembolization versus symptomatic treatment in patients with unresectable hepatocellular carcinoma: a randomized controlled trial. Lancet 2002;359:1734-1739.

6 Kmomorizono Y, Oketani M, Sako k, et al. Risk factors for local recurrence of small hepatocellular carcinoma tumors after a single session, single application of percutaneous radiofrequency ablation. Cancer 2003;97:1253-1262.

7 Kim JW, Kim JH, Won HJ, et al. Hepatocellular carcinoma 2-3 $\mathrm{cm}$ in diameter: transarterial chemoembolization plus radiofrequency ablation vs. radiofrequency ablation alone. Eur J Radiol 2012;81:189-193. 


\section{Curriculum Vitae}

\begin{tabular}{|c|c|}
\hline Name & Jia Fan \\
\hline Position & Professor \\
\hline Institution & $\begin{array}{l}\text { Liver Cancer Institute, } \\
\text { Zhongshan Hospital, Fudan } \\
\text { University }\end{array}$ \\
\hline E-mail & jiafan99@yahoo.com \\
\hline
\end{tabular}

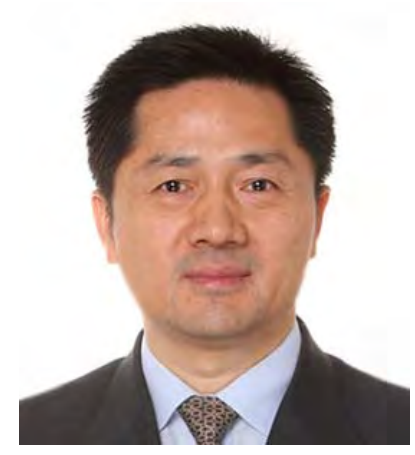

I am vice-president of Zhongshan Hospital, professor and director of department of Liver Surgery and vice-director of department of Oncology and Organ Transplantation Center of Zhongshan Hospital of Fudan University.

In 1988, I received MS degree from Medical College of Southeast University and received my PhD degree in 1995 from Shanghai Medical College of Fudan University. From 1999 to 2000, I had been to University of Pittsburgh Medical Center as visiting scholar. I have authored more than 170 publications, including original articles, editorials, reviews and book chapters. These include the mechanism of liver cancer metastasis, liver cancer microenvironment and liver transplantation.

\section{Research Interest:}

My research work now focuses on the prevention and treatments for recurrence or metastasis of hepatocellular carcinoma after liver resection or transplantation. 


\section{Surgical Treatment for Hepatocellular Carcinoma}

\author{
Jia Fan
}

Liver Cancer Institute, Zhongshan Hospital, Fudan University, Shanghai, China

The incidence of hepatocellular cancer (HCC) has increased worldwide over the past two decades. Surgical resection and liver transplantation have been demonstrated as potentially curative treatment options, which could be considered in $30 \%$ to $40 \%$ of HCC patients. Recent advancements of surgical treatment have focused not only on the surgical techniques, but also the hepatic functional reserve evaluation, resectability assessment and the effects of biological characteristics of tumor on prognosis. There is no single variable to evaluate hepatic functional reserve accurately. Combined Child-Pugh classification, ICG15, portal vein pressure and remanent liver volume measurement are reasonable strategy prior to liver resection. A primary 1 treatment Chinese guideline has been developed, which is based on PS score, liver function and tumor associated variables. 5-year survival rate after liver resection for HCC is about $50 \%$, and the results may be acceptable for some selected patients underwent tumor resection with thrombectomy, including HCC with portal vein tumor thrombus or bile duct thrombosis. The choice of local resection or regular hepatectomy is still controversial although the former is common in HCC with cirrhosis, and the latter is in the setting without liver cirrhosis. The result of liver transplantation for HCC is better than liver resection, and Milan criteria is an generally accepted algorithm. Any attempts to expand the selection criteria should be cautious in the present setting of organ shortage. Salvage transplantation for intra-hepatic recurrence after liver resection may be a good choice in some resectable HCC.

The recurrence and metastasis after surgical treatments are of the main obstacle to achieve better results. Identification of predictive factors could be helpful to develop prevention strategies. Due to the importance of biological characteristics in tumor recurrence and metastasis, a molecular classification to predict prognosis of HCC will lead to a more personalized medicine. Targeting key molecules of biological pathways could optimize therapeutic modality in HCC. 


\section{Curriculum Vitae}

Name

Position

Institution

E-mail
Kyung-Suk Suh

Professor and Chairman

Department of Surgery Seoul National University College of Medicine

kssuh@snu.ac.kr

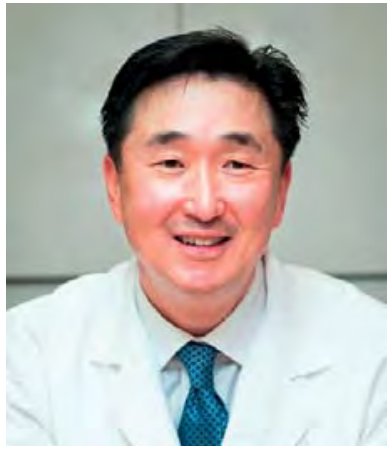

Professor Suh has main interest in liver surgery including liver transplantation. As s pioneer in the field of liver transplantation, he developed many new techniques and published many clinical papers. Now, he is a principal investigator in several nation-wide multi-center studies associated with liver transplantation and surgical treatment of HCC.

\section{Research Interest:}

Prognostic factor and tumor maker for HCC

Correlation between HCC and HBV recurrence

HCC pathogenesis in the fatty liver

Liver transplantation for the advanced HCC 


\section{Expanding Indication: Liver Transplantation for HCC}

Kyung-Suk Suh

Seoul National University Hospital, Seoul, Korea

A living donor is uniquely matched to a certain recipient and this special relationship between a donor and recipient can provide a patient with the opportunity to undergo liver transplantation even in suboptimal patients with advanced hepatocellular carcinoma (HCC). However, a high probability of tumor recurrence should evoke ethical issues concerning risk to the living donor. There are no accepted criteria for patients with tumors that lie outside the conventional criteria (e.g. Milan criteria).

To expand the Milan criteria, prognostic factors other than size and number of tumor may be necessary. Between November 1997 and December 2005, 104 cases of liver transplantation for patients with HCC were performed at our center. Twenty-four patients did not meet the Milan criteria preoperatively. Among these 24 patients, 19 had no major vascular invasion at the time of surgery. We analyzed the survival and prognostic factors of these 19 patients. The mean follow-up period was 33 months (range 6-89). Three-year survival rate in 19 patients was $67.4 \%$. Three-year survival rates were significantly higher when preoperative alpha-fetoprotein was less than $400 \mathrm{ng} / \mathrm{ml}(86.2$ vs. $0 \%, \mathrm{p}<0.001)$ when Edmonson-Steiner's histological grade 1 or 2 (100 vs. $40 \%$, $\mathrm{p}=0.036)$ and when microvascular invasion was absent $(78.6$ vs. $30 \%, p=0.039$ ). Only AFP was a preoperative factor.

Positron emission tomography using F-18 fluoro-2deoxy-d-glucose $\left({ }^{18} \mathrm{~F}\right.$-FDG-PET) imaging is now well established as a noninvasive diagnostic tool for the detection of a variety of malignant tumors. However, in the case of HCC, several investigators have reported controversial conclusions and an inadequate sensitivity for PET (50\%-55\%). Nevertheless, a high positive rate of FDG accumulation has been reported in patients with high-grade HCC and in those with markedly elevated alpha fetoprotein (AFP) levels. When we analyzed the association between tumor factors and PET (+) (greater PET lesion uptake) in liver, preoperative AFP level and vascular invasion were found to be significantly associated with PET $(+)(p=0.003$ and $p<0.001$, respectively). The 2 -yr recurrence-free survival rate (2-yr RFSR) of PET (-) patients was significantly higher than that of PET $(+)$ patients ( $85.1 \%$ vs $46.1 \%$ ) ( $p=0.0005)$. PET imaging could be a good preoperative tool for estimating the post-LT risk of tumor recurrence, because histological grade and vascular invasion cannot be determined preoperatively.

The retrospective data of 178 consecutive HCC patients who underwent LDLT from January 2003 to December 2009 in Seoul National University Hospital were collected. We analyzed 113 patiens who were evaluated all 3 tests including 18F-FDG PET positivity, serum AFP level and serum PIVKA II level preoperatively. Multivariate analysis showed that serum AFP level, serum PIVKA II level and 18F-FDG PET positivity were statistically significant variables. HCC of all 3 patients with high risk of 3 biological factors and within Milan criteria were recurred. However, HCC of only 1 patient among the 9 patients beyond Milan criteria and with low risk of biological factors $(\mathrm{AFP}<100 \mathrm{ng} / \mathrm{mL}$, PIVKA $\mathrm{II}<100 \mathrm{mAU} / \mathrm{mL}$, PET negative) were recurred.

Conclusion: Tumor biological markers have the possibility to overcome the limitation of the Milan criteria. Preoperative serum AFP level, PIVKA II level and 18F-FDG PET positivity of the tumor predict the tumor recurrence better than Milan criteria in living donor liver transplantation for the patients with HCC.

\section{References}

1 Sung Hoon Yang, Kyung-Suk Suh, Hae Won Lee, Eung-Ho Cho, Jai Young Cho, Yong Beom Cho, Ink Hwan Kim, Nam-Joon Yi, Kuhn Uk Lee; A revised scoring system utilizing serum alphafetoprotein levels to expand candidates for living donor transplantation in hepatocellular carcinoma. surgery 2007;141:598-609.

2 Sung Hoon Yang, Kyung-Suk Suh, Hae Won Lee, Eung-Ho Cho, Jai Young Cho, Yong Beom Cho, Nam-Joon Yi, Kuhn Uk Lee; The Role of ${ }^{18} \mathrm{~F}$-FDG-PET Imaging for the Selection of Liver Transplantation Candidates Among Hepatocellular Carcinoma Patients. Liver Transplantation 2006;12:16551660. 


\section{Curriculum Vitae}

$\begin{array}{ll}\text { Name } & \text { Jin Sub Choi } \\ \text { Position } & \begin{array}{l}\text { Professor of Surgery } \\ \text { Institution }\end{array} \\ & \begin{array}{l}\text { Yonsei University College of } \\ \text { Medicine } \\ \text { choi5491@yuhs.ac }\end{array}\end{array}$

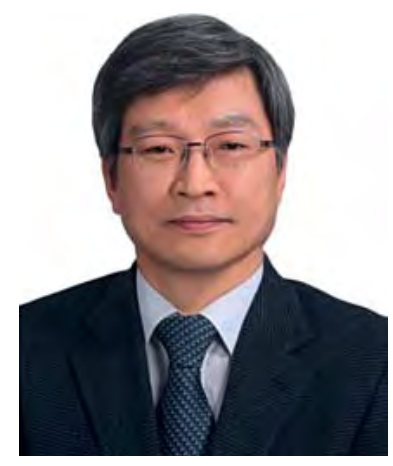

Graduate Yonsei University in 1985.

Internship and surgical resident training in Severance Hospital of Yonsei University Health System.

Visiting Professor, Department of Surgical Immunology and Organ Transplantation, Kyoto University, Kyoto, Japan.

Fellow, Multi-Organ Transplantation Centre, Department of Surgery, Faculty of Medicine University of Toronto, Toronto, Ontario, Canada.

\section{Research Interest:}

Minimally invasive surgery for HCC

Resection of advanced HCC

Liver transplantation 


\section{Surgery for Extensive HCC After Downstaging}

Jin Sub Choi

Department of Surgery, Yonsei University College of Medicine, Seoul, Republic of Korea

Curative aimed surgical treatments such as liver resection, or liver transplantation can provide best survival outcomes for the patients with hepatocellular carcinoma (HCC). However, these curative treatments could be applied only for 10 to $15 \%$ of newly diagnosed patients with HCC, especially in Asia. The natural prognosis of unresectable advanced HCC is very dismal with overall median survival of 3 months only. Moreover, survival outcomes after palliative treatments for unresectable HCC were not satisfactory. However, with improvement of various local and systemic therapies, palliative aimed treatment can downstage tumors from unresectable to resectable and curative aimed treatment can be applied for the patients with advanced HCC. Various downstaging protocols had been introduced including transarterial chemoembolization (TACE), systemic chemotherapy, transarterial ${ }^{90} \mathrm{Y}$ microspheres, transarterial chemotherapy, external beam irradiation combined internal radiation using ${ }^{131}$ I-labeled anti-ferritin, hepatic artery infusion of chemotherapeutic agents sometimes combined with hepatic artery ligation, radioimmunotherapy, and fractionated regional radiotherapy. The proper candidates for liver resection following downstaging should have well preserved liver function and general medical conditions with unresectable HCC. Unresectability might be defined as extensive tumors with insufficient remnant liver volume after liver resection, extensive and multifocal bilobar tumors, and tumors with main portal vein or hepatic vein tumor thrombi. Some investigators reported acceptable survival outcomes after salvage liver resection following downstaging for HCC with five year survival rate from $25 \%$ to $57 \%$. We also experienced 41 cases of salvage liver resection after downstaging of locally advanced HCC. External beam radiotherapy with a concurrent hepatic arterial infusion of 5-fuluorouracil (5-FU) through an implanted percutaneous hepatic arterial catheter following monthly intra-hepatic arterial chemotherapy with 5-FU and cisplatin were used for downstaging modalities. The 5 year overall survival rates were $49.6 \%$. Therefore, liver resection after successful downstaging of unresectable tumor might improve long-term results and increase the possibility of a cure chance in a proportion of patient with advanced HCC. The better tumor downstaging modalities should be further investigated and prospective randomized controlled studies are need be performed to figure out the role of salvage liver resection after tumor downstaging in the patients with unresectable advanced HCC.

\section{References}

1 Padhya KT, Marrero JA, Singal AG. Recent advances in the treatment of hepatocellular carcinoma. Current opinion in gastroenterology 2013;29:285-292.

2 Cabibbo G, Maida M, Genco C et al. Natural history of untreatable hepatocellular carcinoma: A retrospective cohort study. World journal of hepatology 2012;4:256-261.

3 Rahbari N, Mehrabi A, Mollberg NM et al. Hepatocellular carcinoma: current management and perspectives for the future. Annals of surgery 2011;253:453-469.

4 Lau WY, Lai EC. Salvage surgery following downstaging of unresectable hepatocellular carcinoma - a strategy to increase resectability. Annals of surgical oncology 2007;14: 3301-3309.

5 Tang ZY, Zhou XD, Ma ZC, et al. Downstaging followed by resection plays a role in improving prognosis of unresectable hepatocellular carcinoma. Hepatobiliary \& pancreatic diseases international: HBPD INT 2004;3:495498.

6 Lau WY, Ho SK, Yu SC, Lai EC, Liew CT, Leung TW. Salvage surgery following downstaging of unresectable hepatocellular carcinoma. Annals of surgery 2004;240:299-305.

7 Meric F, Patt YZ, Curley SA et al. Surgery after downstaging of unresectable hepatic tumors with intra-arterial chemotherapy. Annals of surgical oncology 2000;7:490-495.

8 Majno PE, Adam R, Bismuth H etal. Influence of preoperative transarterial lipiodol chemoembolization on resection and transplantation for hepatocellular carcinoma in patients with cirrhosis. Annals of surgery 1997;226:688-701. 


\section{Curriculum Vitae}

$\begin{array}{ll}\text { Name } & \text { Xiao-Ping Chen } \\ \text { Position } & \begin{array}{l}\text { Professor and Chairman } \\ \text { Institution }\end{array} \\ & \begin{array}{l}\text { Department of Surgery, Tongji } \\ \text { Hospital } \\ \text { chenxpchenxp@163.com }\end{array}\end{array}$

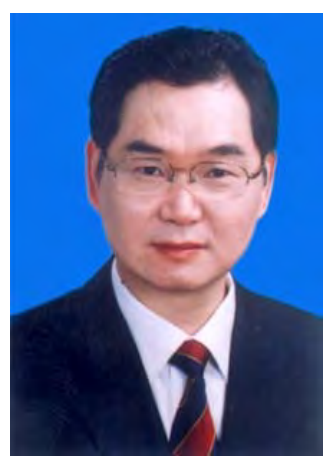

Prof. Xiao-Ping Chen is Chairman of Department of Surgery, Director of Institute of Organ Transplantation and Director of Institute of Hepato-PancreatoBiliary Surgery of Tongji hospital. He is an executive member of International Hepato-Pancreato-Biliary Association and Chairman of the Chinese Chapter, president of Asia-Pacific Hepato-Pancreato-Biliary Association. He is also a fellow of the American College of Surgeons and Honorary Fellowship of America Surgical Association.

Prof. Chen has devoted himself to clinic work, teaching, and research for over 30 years. He has performed more than 10 thousand hepato-biliary operations, in which over 6000 cases are liver resections, and achieved very good clinical outcomes. He established some important theories and improved a lot of surgical techniques of liver surgery.

He is editor-in-chief or editor for more than 50 kinds of national and international journals, has more than 500 papers published in national and international journals, the papers have been cited for over 2000 times.

\section{Research Interest:}

Hepatocellular carcinoma, Liver transplantation, Cell signaling pathway, Cancer metastasis, Hilar cholangiocarcinoma, Portal hypertension, Pancreatic cancer, Cellular immunity, Cell metabolism. 


\section{Surgery for Extensive HCC without Downstaging}

\section{Xiao-Ping Chen}

Department of Surgery, Tongji Hospital, Wuhan, China

Hepatocellular carcinoma (HCC) commonly occurs after infection with hepatitis B and C in Africa and Asia, and its incidence is also increasing in Western countries. In general, tumour size is a major determinant of the treatment of choice for HCC. Liver transplantation has proved an effective treatment only for small HCC in patients with cirrhosis. And some investigators have indicated that transcatheter arterial chemoembolization (TACE) rarely provides complete necrosis of large tumours.

We have a retrospective study evaluated whether the long-term outcome of hepatectomy for large HCC improved over 14 years in one centre. Data from 2102 patients who underwent hepatectomy for large HCC were collected prospectively and divided into two groups (group 1 includes 959 cases, group 2 includes 1143 cases). Cumulative 1-, 3- and 5-year overall survival rates were 71.2, 58.8 and 38.7 per cent respectively in group 2 , and were significantly better than respective rates of $67.8,50.7$ and 27.9 per cent in group 1 . Cumulative 1-, 3- and 5-year disease-free survival rates were $61.5,38.6$ and 23.8 per cent respectively in group 2 , and 56.5 , 34.7 and 18.9 per cent in group 1 . There was a significant difference in median survival time after recurrence between groups 2 and 1 (17 and 10 months respectively; $\mathrm{P}=0.043$ ).

Hepatic resection in patients with large HCChas improved overall and disease-free survival during the past decade at our institute. Long-term survival can be improved significantly by aggressive treatment of recurrent tumours. Accordingly, surgical resection is still considered the most effective treatment for HCC, even though the potential benefits of partial resection have not been fully delineated. Over the past decade, improvements in resection techniques and perioperative management have reduced operative mortality in patients with HCC. 


\section{Curriculum Vitae}

\begin{tabular}{|c|c|}
\hline Name & Dr. Stephanie Ma \\
\hline Position & Assistant Professor \\
\hline Institution & Department of Clinical Oncology \\
\hline & $\begin{array}{l}\text { State Key Laboratory for Liver } \\
\text { Research }\end{array}$ \\
\hline & Faculty of Medicine \\
\hline E-mail & $\begin{array}{l}\text { The University of Hong Kong } \\
\text { stefma@hku.hk }\end{array}$ \\
\hline
\end{tabular}

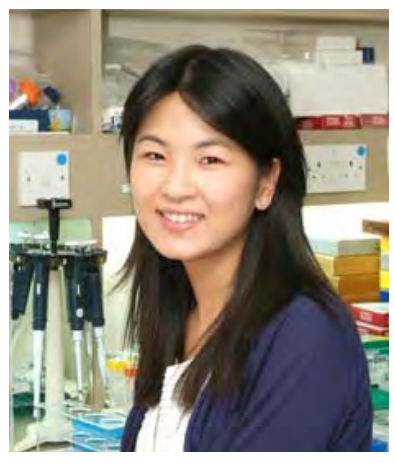

Dr. Stephanie Ma graduated from the University of British Columbia, Canada with a B.Sc. degree in Cell Biology and Genetics in 2000 and a M.Sc. degree in Pathology and Laboratory Medicine in 2003. She then returned to Hong Kong and pursued a Ph.D. degree at the University of Hong Kong under the supervision of Prof. Xin-Yuan Guan and Dr. Kwok Wah Chan. She successfully completed her doctorate thesis which was focused on the identification and characterization of liver cancer stem cells in 2007 and was awarded with the Li Ka Shing Prize for the Best Ph.D. Thesis of the year. Following graduation, Dr. Ma trained as a postdoctoral fellow in the Departments of Clinical Oncology and Pathology and was subsequently promoted to a Research Assistant Professor position two years later in the same institution. Dr. Ma is currently an Assistant Professor in the Department of Clinical Oncology at The University of Hong Kong. She is also a member of the State Key Laboratory of Liver Research. Dr. Ma is the recipient of numerous awards for her outstanding achievements in liver cancer research work, including the 2008 Hong Kong Young Scientist Award in Life Sciences (Hong Kong Institution of Science) and the 2011 State Key Laboratory for Liver Research - Young Researcher Career Development Scheme. She is also one of the top $1 \%$ most cited scholars in "Clinical Medicine" and "All Fields" as ranked by the ISI's Essential Science Indicators.

\section{Research Interest:}

Dr. Ma's area of research interests is primarily focused on the identification, characterization, omic profiling and therapeutic targeting of hepatic cancer stem cells as well as cancer epigenetic and molecular pathogenesis of liver cancer. She was one of the first to identify CD133 as a functional liver cancer stem cell marker and has also helped characterize the molecular mechanisms by which this subset of cells drives hepatocellular carcinoma. Dr. Ma has published extensively in the area of hepatocellular carcinoma research with her findings published in leading journals including, but not limited to, Cell Stem Cell, Gastroenterology, Hepatology, Cancer Research, Oncogene, etc. 


\section{Role of Liver Cancer Stem Cells in HCC Metastasis}

Stephanie $\mathrm{Ma}$

Department of Clinical Oncology, State Key Laboratory for Liver Research, Faculty of Medicine, The University of Hong Kong, Pok Fu Lam, Hong Kong

The recent emergence of the cancer stem cell (CSC) concept lends insight into the explanation of why treatment with chemotherapy often may seem to be initially successful but results in not only a failure to eradicate the tumor but also possibly tumor relapse. Commonly used anti-cancer drugs in
HCC work by targeting the rapidly proliferating and differentiated liver cancer cells that constitute the bulk of the tumor. However, a subset of CSCs exists within the tumor, which are more resistant and are able to survive and maintain residence after treatment, thus, growing and self-renewing to generate the development and spread of recurrent tumors in HCC. In the past few years, compelling evidence has emerged in support of the hierarchic CSC model for solid tumors, including HCC. And in particular, CD133 has drawn significant attention as a critical functional liver CSC marker. In this talk, a summary of our recent work in $\mathrm{CD} 133^{+}$liver CSC research with regard to identification, characterization and regulation will be discussed. 


\section{Curriculum Vitae}

$\begin{array}{ll}\text { Name } & \text { Hiromitsu Hayashi } \\ \text { Position } & \text { Assistant Professor } \\ \text { Institution } & \text { Kumamoto University } \\ \text { E-mail } & \text { h.hayashi13@gmail.com }\end{array}$

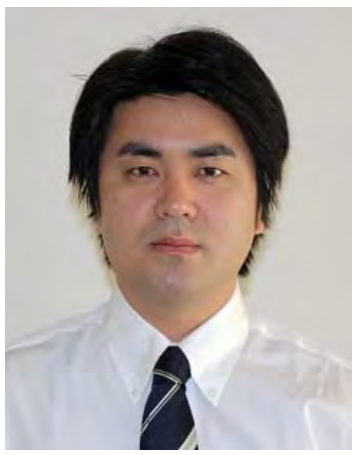

2001 M.D. Kumamoto University, Japan

2009 Ph.D. (Dr. of Medical Science) Kumamoto University, Japan

2009 Postdoctoral research fellow, Cleveland Clinic (Ohio, USA)

2012-present Assistant professor, Department of Gastroenterological Surgery, Kumamoto University

DDW2012 Poster of Distinction

\section{Research Interest:}

My scientific interests are in the role of TGF- $\beta$ signal in tumor progression and liver regeneration.

TGF- $\beta$ has a variety of biological effects such as cell cycle arrest, apoptosis, EMT, and ECM deposition. Indeed, TGF- $\beta$ signal plays a crucial role in cancer progression. We provided the evidence that TGF- $\beta$-mediated CD44 induction was required for the EMT in HCC (Mima K, Hayashi H, et al. Cancer Res, 2012).

Furthermore, we displayed that thrombosponidin-1-medaited TGF- $\beta$ local activation mechanism played a negative role in liver regeneration after hepatectomy (Hayashi et al. Hepatology, 2012).Thus, we are now encouraged to investigate the role of TGF- $\beta$ signal in HCC progression and liver regeneration. 


\section{CD44s Requires for TGF- $\beta$-Mediated Mesenchymal Phenotype and Is Associated with Poor Prognosis in Patients with Hepatocellular Carcinoma}

Hiromitsu Hayashi, Kosuke Mima, Takatsugu Ishimoto, Hirohisa Okabe, Shigeki Nakagawa, Hideyuki Kuroki, Takatoshi Ishiko, Masayuki Watanabe, Toru Beppu, Hideo Baba

Department of Gastroenterological Surgery, Graduate School of Medical Sciences, Kumamoto University,

1-1-1 Honjo, Kumamoto, Japan

The prognosis for individuals diagnosed with hepatocellular carcinoma (HCC) remains poor due to the high frequency of invasive tumor growth, intrahepatic spread, and extrahepatic metastasis. CD44, a major adhesion molecule of the extracellular matrix, has been implicated in a wide variety of physiological processes, including leukocyte homing and activation, wound healing and cell migration. Recently, cancer stem cells in many tumors have been identified by positive expression of CD44, either individually or in combination with other markers and these cells have been shown to be involved in tumor progression and metastasis. Here, we investigated the role of the standard isoform of CD44 (CD44s) in the TGF- $\beta$ mediated mesenchymal phenotype of HCC. Then, CD44s was the dominant form of CD44 mRNA expressed in HCC cells. The overexpression of CD44s promoted tumor invasiveness and increased the expression of vimentin, a mesenchymal marker, in HCC cells. The treatment with TGF- $\beta 1$ induced the mesenchymal phenotype of HCC cells, which was characterized by low E-cadherin and high vimentin expression. Loss of CD44s abrogated these changes. Clinically, the overexpression of CD44s was associated with low expression of E-cadherin, high expression of vimentin, a high percentage of phospho-Smad2positive nuclei, and resulted in poor prognosis in HCC patients. Furthermore, the overexpression of CD44s was also highly detected in locally recurrent HCC after local ablation therapy which is a widely used treatment for HCC, and was associated with aggressive recurrence patterns such as the intrahepatic dissemination of HCC. Taken together, our findings suggest that CD44s plays a critical role in the TGF- $\beta$-mediated mesenchymal phenotype and thereby participates in tumor progression in HCC. 


\section{Curriculum Vitae}

$\begin{array}{ll}\text { Name } & \text { Seung Kew Yoon } \\ \text { Position } & \text { Professor } \\ \text { Institution } & \text { The Catholic University of Korea } \\ \text { E-mail } & \text { yoonsk@catholic.ac.kr }\end{array}$

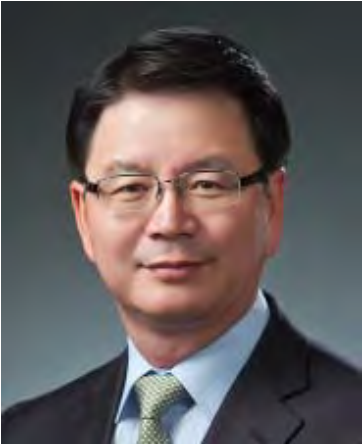

I am a professor at division of Hepato-gastroenterology in Seoul St.Mary Hospital, The Catholic University of Korea. And I am a director of Catholic University Liver Research Center with WHO Collaborating Center of Viral Hepatitis in Korea. I graduated from Catholic University Medical College, then went on to completed my M.S. and Ph.D. at the postgraduate School. In 1996-1998, I studied abroad as a research fellow of Cancer center at Massachusetts General Hospital, Harvard Medical School, USA. I was a ex-scientific director of Korean Association of Study of Liver and Korean Association of Gastroenterology.

\section{Research Interest:}

Hepatocarcinogenesis, Cancer Stem Cell, Fibrogenesis, Hepatitis C Virus, Tumor marker. 


\section{Mechanism of Acquired Chemoresistance in HCC}

\author{
Seung Kew Yoon \\ Division of Hepato-Gastroenterology, Department of \\ Internal Medicine, College of Medicine, The Catholic \\ University of Korea, Seoul, Korea
}

Despite recent advances in treatment of hepatocellular carcinoma (HCC), options for curing the disease are limited. In cases of advanced HCC, anti-cancer chemotherapeutic drugs have been used; however, data demonstrating their efficacy in patients is limited. The reason why systemic and loco-regional anti-cancer therapy against HCC fail is largely due to the acquired chemoresistance to drug. Recently, a number of molecular mechanisms of chemoresistance in HCC including tumor hypoxia, expression of DNA repair enzymes, abrogation of apoptosis signaling and altered miRNA expression have been reported. We recently demonstrated that overexpression of 14-3-3 3 protein was associated with the chemoresistance to cis-diammined dichloridoplatium (CDDP). However, the precise mechanism underlying chemoreistance is not completely understood. Furthermore, the exact reason for why the response to anti-cancer therapies was different even in the same type of cancer from different patients remains unclear.

Cancer stem cell (CSC) is defined as a small population of cells within a tumor that possess the capability for selfrenewal and generation of heterogenous lineages of cancer cells. Because the biological and molecular features of cancer stem cells (CSCs) within tumors, including diverse regulatory mechanisms and signaling pathways, may contribute to cancer cell survival, it might explain the reason why conventional anti-cancer therapies have shown such resistance. It has been recognized that CSCs containing a quiescent nature have inherent resistance to conventional therapies. Furthermore, once chemotherapy-resistant CSCs re-enter the cell cycle, they generate rapidly dividing progenitor cells with the ability to reestablish the tumor. In addition, recent works have suggested several mechanisms for chemoresistance by CSCs. Adenosine triphosphate (ATP)-binding cassette (ABC) transporters commonly expressed on cellular membranes of both normal stem cells and CSCs include multidrug resistance transporter 1 (MDR1) and breast cancer resistance protein (BCRP) drug transport pumps and play an essential role in expelling anticancer drugs from cells, leading to chemoresistance. Another study reported on involvement of CSCs expressing aldehyde dehydrogenase 1 (ALDH1), which facilitates metabolism, in chemoresistance. Huh7 side population (SP) cells expressing ABC transporters, such as MDR1 and $B C R P 1$, showed chemoresistance to doxorubicin. In addition, Huh7 SP cells were found to overexpress CEACAM6, which was known to be associated with chemoresistance to gemcitabine. Regarding the role of CD133 in development of HCC, CD133-expressing cancer cells are not only responsible for tumor initiation or progression, but also have stem celllike properties, like colony-forming ability and differentiation potential. Moreover, recent report showed that CD133+ HCC cells contribute to chemoresistance through preferential activation of Akt/PKB and Bcl-2 cell survival response. More recently, CD13 was reported to be a marker for semiquiescent CSCs in human liver cancer cell lines and that CD13 predominated in the $\mathrm{G}_{0}$ phase of the cell cycle, decreased ROS-induced DNA damage after genotoxic chemo/radiation stress, and protected cells from apoptosis. Recent studies have suggested that last surviving cancer stem cells during anti-cancer chemotherapy can lead to tumor regrowth or recurrence via their enhanced chemoresistance. Therefore, understanding the mechanisms by which chemoresistance can occur is important to developing novel therapeutic approaches to treating HCC. 


\section{Curriculum Vitae}

$\begin{array}{ll}\text { Name } & \text { Yun-Han Lee } \\ \text { Position } & \text { Assistant Professor } \\ \text { Institution } & \begin{array}{l}\text { Department of Radiation Oncology, } \\ \text { College of Medicine, Yonsei }\end{array} \\ & \begin{array}{l}\text { University } \\ \text { yhlee87@yuhs.ac }\end{array}\end{array}$

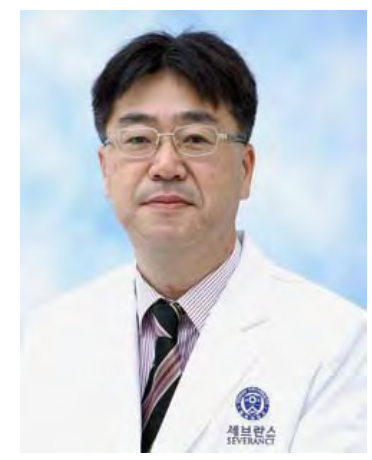

My research program pursues to discovery of druggable targets through functional genomic studies and its translation into the development of cancer therapeutics with molecular medicine, particularly to hepatocellular carcinoma (HCC). Functional validations of targets in vitro and in vivo are carried out by employing functional genomics tools such as microarray and RNAi technology, in vitro phenotypic assays, orthotopic xenograft mouse models and in vivo delivery of modified target siRNA by SNALP system. In vivo efficacy of target gene silencing is estimated by the observations of tumor relapse with bioluminescence imaging, histological examination and survival analysis. Global transcriptomic analysis of target gene knockdown signature and subsequent proteomic analysis (e.g. an extensive western blotting) are also performed to gain a better understanding of key molecular alterations underlying the observed tumor growth suppression, thereby simultaneously providing action mechanism of a molecular targeted medicine. While my primary research program involves discovery of target and development of molecular cancer therapeutics, I am also interested in extending my works to other disciplines such as therapeutic targeting of cancer stem cells, combination therapy, and identification of small molecule mechanisms and therapeutic potential.

\section{Research Interest:}

1 Functional validation of therapeutic target genes or pathways using gene knockdown technology such as RNAi.

2 Elucidation of molecular mechanisms underlying cancer progression or regression.

3 Development of molecular cancer therapeutics.

4 Exploitation of combined therapy of targeting more than one functional pathway for an improved therapeutic index.

5 Therapeutic targeting of cancer stem cells (CSC) in solid tumor for the prevention of tumor recurrence or metastasis and the reduction of radioresistance. 
Radio-Resistance of Cancer Stem Cell in HCC Yun-Han Lee

Department of Radiation Oncology, College of Medicine, Yonsei University, Seoul, Republic of Korea

Hepatocellular cancer (HCC) is the third most common cause of cancer death worldwide. It ranks sixth worldwide in terms of incidence, and it is particularly common in developing countries. Rates of HCC diagnosis are increasing in many parts of the world, including the United States. The majority of patients diagnosed with HCC are not eligible for radical curative therapy, and median survival for such patients is less than one year.

Ionizing radiotherapy (RT), which plays a major role in the management of most solid tumors including HCC. Meanwhile, it is well known that the presence of a small number of cancer stem cells (CSCs) in the heterogenous cancer cell population can be ultimately responsible for tumor initiation as well as tumor recurrence and metastasis. Study of CSC biology is one of the most thriving and competitive areas in radiation oncology research as well because it has the potential to dramatically affect clinical outcomes. As RT is only second to surgery or chemotherapy in terms of its curative potency, it is very important for radiation oncologist to learn whether progress in CSC biology can enable them to exploit this knowledge to help cure more patients suffering from cancer.

CSCs have been identified in many tumor types, including HCC with observable heterogeneity. Numerous studies have shown that hepatic CSCs could be enriched and isolated via different cell surface markers, such as CD13, CD24, CD44, CD90, CD133, EpCAM (CD326) and OV6. CSCs are also resistant to conventional radio-therapies. A recent study proved that ionizing radiation can induce stem-cell like properties in heterogenous non-stem cancer cells by enhancing spherogenesis and upregulating the cancer stemness genes Sox 2 and Oct3/4, defining a novel mechanism of resistance of cancer cells to radiotherapy.

While the multi-targeted small molecule tyrosine kinase inhibitor sorafenib has been shown to prolong overall survival in advanced HCC, it yields low radiographic response rates, suggesting that sorafenib is suboptimal as local therapy. Thus, development of novel options of targeted therapy for HCC remains a major challenge. Functional characterization of hepatic CSCs has revealed several deregulated signaling pathways, such as Wnt/ $\beta$-catenin, AKT, TGF- $\beta$, IL-6/STAT3 pathways to be critical in inducing "stemness" of HCC and in promoting self-renewal, tumorigenecity and resistance to treatment.

An increased understanding of hepatic CSC biology will be able to facilitate the development of new therapeutic strategies for improving HCC clinical management. In the near future, in terms of developing novel multimodality treatment regimens, small molecules targeting CSC biology-related genes could be discovered, and then be utilized as radiosensitizing agents by combining it with HCC radiation therapy. 


\section{Curriculum Vitae}

$\begin{array}{ll}\text { Name } & \text { Masatoshi Makuuchi } \\ \text { Position } & \begin{array}{l}\text { President } \\ \text { Institution }\end{array} \\ & \begin{array}{l}\text { Japanese Red Cross Medical } \\ \text { Center } \\ \text { masatoshi.makuuchi@ } \\ \text { gmail.com }\end{array}\end{array}$

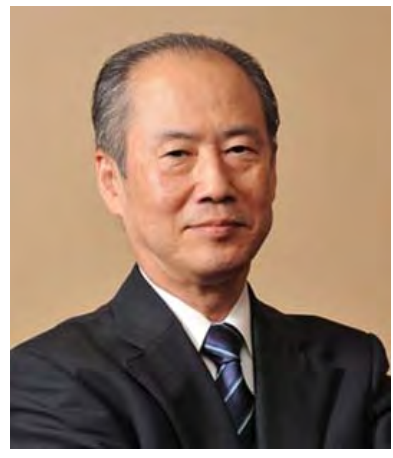

A pioneer and a recognized international expert in the field of HBP surgery, Dr. Makuuchi is currently the President of the Japanese Red Cross Medical Center, and the Professor Emeritus of the University of Tokyo.

Throughout his career, Dr. Makuuchi has taken initiatives in developing methods of perioperative managements such as ultrasound guided PTC, BTBD, GB drainage, PVE, intermittent hemihepatic inflow occlusion, and warm ischemia in living donor, and surgical techniques such as subsegmentectomy, and inferior hepatic vein preserving hepatectomies, contributing to establish techniques for safe surgical operation. He is also the first in the world to successfully perform adult to adult LDLT. 


\section{Past, Present and Future of Surgical Resection for HCC}

\section{Masatoshi Makuuchi}

President, Japanese Red Cross Medical Center, Professor Emeritus, The University of Tokyo, Tokyo, Japan

Today, surgical resection is an extremely effective treatment option in locally controlling hepatocellular carcinoma (HCC), and by the continuous challenges of the liver surgeons, its short- and long-term results have improved dramatically in the recent years. In order to systematically eradicate the minute intrahepatic metastases together with the main tumor, anatomic resection is useful and effective. For HCC with tumor thrombus, surgical resection following transcatheter hepatic arterial chemoembolization (TACE) is able to provide satisfactory survival, and when large amount of liver parenchyma is to be removed, portal vein embolization (PVE) is a useful technique to induce hypertrophy of future remnant liver.
While high recurrence rate after curative resection remains to be a serious problem, liver functional reserve permitting, repeated surgical resection is possible and is effective. At the same time, establishing an effective adjuvant therapy to prevent the recurrence is being sought for.

Liver transplantation (LT) is an ideal treatment in replacing the pre-cancerous injured liver with HCC. However, shortage of deceased donors, indismissible operative mortality rate, extremely long waiting list and post-transplant recurrence are still very serious. Although Milan criteria remains to be the gold standard, and is sometimes an obstacle to apply the treatment for HCC patients, LT may become more popular for HCC patients in the future. In living donor liver transplantation (LDLT), liver graft supply has always been the problem. However, the introduction of surgical techniques for venous reconstruction and the use of various partial grafts now makes LDLT a serious treatment option.

In conclusion, surgery, both resection and transplantation, will continue to be the first-line therapeutic modality for HCC. 


\section{Curriculum Vitae}

$\begin{array}{ll}\begin{array}{l}\text { Name } \\ \text { Position }\end{array} & \begin{array}{l}\text { Riad Salem } \\ \text { Chief, Interventional Radiology } \\ \text { Vice-Chair, Image-Guided Therapy }\end{array} \\ \text { Institution } & \begin{array}{l}\text { Northwestern University } \\ \text { Chicago, IL }\end{array} \\ \text { E-mail } & \text { r-salem@northwestern.edu }\end{array}$

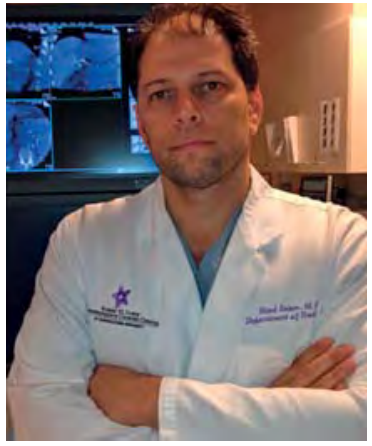

I am Chief of Interventional Radiology at Northwestern University in Chicago. I am originally from Montreal and completed my training at McGill. I completed my IR fellowship at the University of Pennsylvania. I also have a Master's of Business Administration degree with concentration in finance.

\section{Research Interest:}

My interests are in locoregional treatments and image response assessment in HCC. I am participating in 3 international randomized trials involving radioembolization (HCC: STOP-HCC, YES-p, colorectal: EPOCH). 


\section{Radioembolization with Yttrium 90} Microspheres for Hepatocellular Carcinoma

Riad Salem

Professor of Radiology, Medicine and Surgery Chief, Section of Vascular and Interventional Radiology Director, Interventional Oncology, Vice-Chairman, Image-Guided Therapy, Department of Radiology, Northwestern University, Chicago, IL, USA

Treatment options for liver tumors that cannot be resected are based on trans-arterial techniques. 90Y microspheres represents one of those trans-arterial options. During the past 10 years, numerous studies involving larger cohorts, comparative effectiveness and small randomized studies have provided evidence of the safety and efficacy of 90Y in HCC. The versatility of this therapy is also highlighted in with selective lobar/segmental infusion with the intent of preserving functional liver reserve, downstaging to resection, radiofrequency ablation or liver transplantation. Of recent significant interest are radiation segmentectomy and lobectomy, treatment options that challenge RFA and PVE in HCC. The role in portal vein thrombosis is also of significant clinical interest. This presentation will review the state of the science and present role of radioembolization in HCC. 


\section{Curriculum Vitae}

$\begin{array}{ll}\text { Name } & \text { Jordi Bruix } \\ \text { Position } & \text { Senior Consultant } \\ \text { Institution } & \text { Hospital Clinic - Barcelona } \\ \text { E-mail } & \text { jbruix@clinic.ub.es }\end{array}$

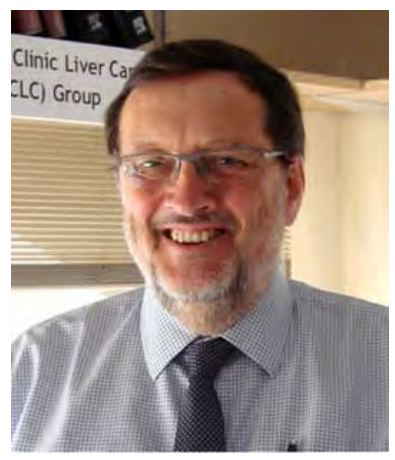

Jordi Bruix, MD is Professor of Medicine at the University of Barcelona and Director of the Barcelona Clinic Liver Cancer Group within the Liver Unit at the Hospital Clinic of Barcelona, Spain.

Dr. Bruix is member of the European Association for the Study of the Liver (EASL) and the American Association for the Study of Liver Diseases (AASLD). He founded the International Liver Cancer Association and was nominated President from 2006 to 2009. He has been Associate Editor of Journal of Hepatology, Liver Transplantation and Hepatology, and is currently Associate Editor of Lancet Oncology and Seminars in Liver Disease., while also being reviewer for several journals and official agencies in USA, Europe and Asia.

He has developed the BCLC staging and treatment strategy that has been endorsed by several international scientific associations to guide management of patients with HCC. Dr. Bruix has authored more than 150 original investigations and led the Evidence-based Practice Guidelines for Hepatocellular Carcinoma at EASL, AASLD and WGO, as well as consensus statements to define endpoints in clinical trials that have paved the conduct and analysis of such investigations.

\section{Research Interest:}

Dr. Bruix has been principal investigator of studies and clinical trials that have changed practice in the field of HCC, this including development of diagnostic criteria, prognostic models and establishing Chemoembolization and sorafenib as conventional therapy. 


\section{Drug Eluting Microsphere TACE (DC Beads) Jordi Bruix \\ BCLC Group, Liver Unit. Hospital Clinic, University of Barcelona, Barcelona, Spain}

Transarterial chemoembolization (TACE) is now an established treatment option for patients with hepatocellular carcinoma who are not candidates for surgery or ablation and that have not reached an advanced stage because of vascular invasion or extrahepatic spread, or because of relevant cancer symptoms. This profile defines the BCLC B stage patients. For an optimal efficacy of TACE the selection of patients is of paramount importance, but it is also relevant to define when TACE is no longer efficacious and should not be repeated. This so called "untreatable progression" because of treatment failure or appearance of an adverse clinical profile should prime to shift the patients to systemic therapy, currently sorafenib.

It is well known that application of TACE has major heterogenity between groups. Until very recently, the most common approach was to inject an emulsion of lipiodol and chemotherapy (usually doxorubicin or cisplantin) and then obstruct the arterial vessels feeding the tumor by injection of gelfoam fragments. Unfortunately, the lipiodol emulsion did not achieve retention of chemotherapy within the tumor and drug accessed the systemic circulation with emergence of chemotherapy adverse events. In addition, gelfoam fragments were not homogeneous and this also impacted in the quality of arterial obstruction.

The development of DCBeads that have an accurately callibrated size and are charged with doxorubicin that is slowly released into the blood upon injection, has sharply improved the technical application of TACE with significant reduction of chemotherapy related adverse events. Therapeutic efficacy in terms of necrosis is maintained if not increased and, as a result, the TACE technique is no longer an artisanal approach with excessive variation.

Several studies have shown the safety of TACE using DCBeads and current data show that the media survival of patients with HCC treated with this technique is around 48 months (Burrel et al, J Hepatol 2012). This data show that TACE may even compete with surgical resection in patients with multifocal disease. 


\section{Curriculum Vitae}

$\begin{array}{ll}\text { Name } & \begin{array}{l}\text { Han Chu Lee } \\ \text { Position }\end{array} \\ \text { Professor, Dept. of Internal } \\ \text { Medicine } \\ \text { Institution } & \begin{array}{l}\text { Asan Medical Center, } \\ \text { University of Ulsan College } \\ \text { of Medicine } \\ \text { hch@amc.seoul.kr }\end{array}\end{array}$

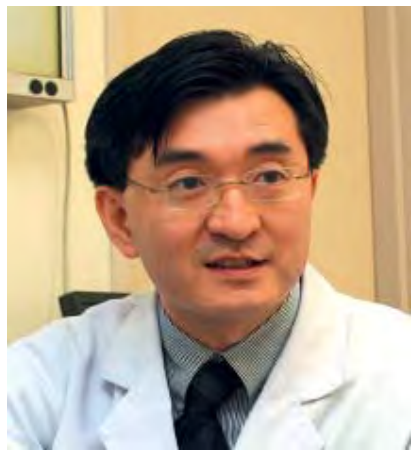

Dr. Han Chu Lee is currently a professor of the Department of Gastroenterology at Asan Medical Center, University of Ulsan College of Medicine, having been an associate professor from 2005 to 2009 at Asan Medical Center, University of Ulsan College of Medicine. He also has an experience to instruct at Ewha Woman's University from 1997 to 1999.

He graduated from College of Medicine, Seoul National University in 1988, and went through the internship and the residency courses until 1992 at Seoul National University Hospital. After getting master's degree in 1997 and doctor's degree in 2003, he got a postdoctoral research fellowship at Liver research Center, Brown University RI Hospital in 2006.

\section{Research Interest:}

Hepatocellular carcinoma 


\section{How to Predict and Evaluate Tumor Response to TACE?}

Han Chu Lee

Department of Internal Medicine, Asan Medical Center, University of Ulsan College of Medicine, Seoul, Korea

Overall survival is, of course, the most important endpoint in oncological practice. However, it cannot be used as a reliable endpoint in phase 2 studies since it cannot be properly powered due to the relatively small sample size. Therefore, other indirect surrogate endpoints should be used for evaluating the efficacy of a specific treatment in earlier phase clinical trials.

'Response rate' has long been used as a primary endpoint for phase 2 studies, but, with the advent of molecular targeted agents, the reliance on response rate is now questioned since it can underestimate the benefits of molecular targeted agents including sorafenib. However, TACE exerts its anti-tumor effect by inducing ischemic necrosis of the tumors, and thereby 'tumor response' still has room for being used for evaluating anti-tumor effect of TACE.

However, there have been several unresolved issues in evaluating anti-tumor effect of TACE by tumor response analyses. First, conventional response assessments such as WHO criteria or the RECIST evaluation mainly focus on shrinkage of the entire tumor and do not consider ischemic necrosis in tumors. To correct for underestimation of the real response rate with use of the original size criteria, EASL or modified RECIST (mRECIST) criteria, which assess only viable tumors bidimensionally or unidimensionally, respectively, have been proposed. However, until recently, few studies have evaluated which response criterion can predict the patient's survival after TACE best. Second, few studies have clearly demonstrated that the lipiodol-deposited area are truly necrotic after lipiodol-based TACE. And also, the beamhardening artifact by lipiodol can mask a viable portion of the tumor on the CT scan. Third, poor hepatic reserve function can limit performing further TACE procedure, and also is an important factor determining patient's survival. Therefore, response to TACE in Child B patients could be lower not because of resistance of tumor cells to TACE but because of incapability to perform further TACE (a situation similar to guarantee-time bias).

To answer these questions, we first evaluated whether the measurement of viable tumor portions after TACE by dynamic CT scan correlates with the measurement by dynamic MRI or pathological measurement. For this purpose, we evaluated the tumor response after TACE in patients who underwent surgical resection or liver transplantation after TACE. Our data clearly showed that response estimation based on measurement of lipiodol deposits as necrosis on CT correlated with MR-based evaluation and actual pathological response. $^{1}$
Second, we retrospectively analyzed the relationship between response to TACE and patient's survival. Since TACE is mostly indicated for BCLC B patients, we excluded BCLC A or $C$ patients who received TACE to avoid the impact of tumor stage on patient's survival. We also excluded patients in Child $\mathrm{B}$ or $\mathrm{C}$ to minimize the impact of hepatic reserve function on performing further TACE procedure or patient's survival. Among the 4 response evaluation methods, the tumor response according to the mRECIST criterion best correlated with patient's survival. mRECIST also has an advantage that it is easy to be applied and less prone to inter-observer variability and measurement bias, compared to EASL criterion. The mRECIST assessment also showed a clear correlation between tumor response and time-to-progression, another important endpoint for phase 2 trials widely used nowadays. ${ }^{2}$ We also evaluate the relationship between the tumor response and survival using the landmark method to avoid the possible guarantee-time bias. However, the guarantee-time bias did not work in our study populations, since most patients had good hepatic reserve function and few patients died within 6 months after first TACE. In fact, the best tumor response correlated better with patient's survival than response after $1^{\text {st }}$ TACE did (unpublished data).

For multiple tumors, we demonstrated that evaluating the largest two lesions is sufficient for measuring TACE responses under EASL and mRECIST, which can make us to avoid the tedious effort to measure all the measurable lesions. ${ }^{3}$

We recently analyzed which variables before treatment can predict the patient's survival after TACE. Since TACE is sometimes performed in BCLC A or C patients as well as B patients, we included all the patients who were treated by TACE. Six independent parameters were identified and we developed a nomogram for patient's survival after TACE using these 6 parameters (unpublished data).

In conclusion, tumor response to TACE by mRECIST best correlates with patient's survival and time-to-progression after TACE. Therefore, tumor response by mRECIST can still be used as an indirect endpoint for clinical trials using TACE.

\section{References}

1 Shim JH, Han S, Shin YM, et al. Optimal Measurement Modality and Method for Evaluation of Responses to Transarterial Chemoembolization of Hepatocellular Carcinoma Based on Enhancement Criteria. J Vasc Interv Radiol 2013;24:316-325.

2 Shim JH, Lee HC, Kim SO, et al. Which Response Criteria Best Help Predict Survival of Patients with Hepatocellular Carcinoma Following Chemoembolization? A Validation Study of Old and New Models. Radiology 2012;262:708718.

3 Shim JH, Lee HC, Won HJ, et al. Maximum number of target lesions required to measure responses to transarterial chemoembolization using the enhancement criteria in patients with intrahepatic hepatocellular carcinoma. J Hepatol 2012;56:406-411. 


\section{Curriculum Vitae}

$\begin{array}{ll}\text { Name } & \text { Peter Robert Galle } \\ \text { Position } & \text { Direktor 1st Medical Department } \\ \text { Institution } & \text { Unimedizin Mainz } \\ \text { E-mail } & \text { galle@uni-mainz.de }\end{array}$

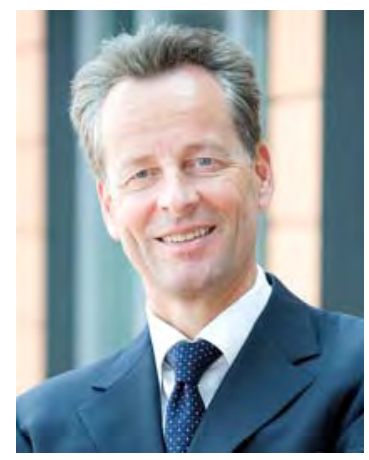

Dr. Galle majored in internal medicine at the Universities of Berlin and Marburg/Germany, Hammersmith Hospital, London/UK and University of Texas/USA and received his M.D. degree from Marburg University and Ph.D. degree from Heidelberg University.

Initially he held a position as postdoctoral fellow in Molecular Biology at the Centre for Molecular Biology Heidelberg working on the replication of hepatitis $B$ viruses. Afterwards he completed his residency in Internal Medicine and Gastroenterology at the University Hospital of Heidelberg. In 1998 he became Director of the I. Medical Department in Mainz and from 2005 - 2008 he hold the CEO position of Mainz University Hospital.

He is member of several national and international societies such as the American Association for the Study of Liver Diseases (AASLD) and the European Association for the Study of the Liver (EASL), and serves as Co-editor for the Journal of Hepatology and is on the Editorial Boards of several other Journals. He is member of the Executive Board and President-Elect of the International Liver Cancer Association (ILCA).

\section{Research Interest:}

His research has focused on elucidating important aspects of apoptotic cell death in the liver and immune escape of tumour cells. He was awarded several prizes, amongst others the prestigious Tannhauser award, the highest prize of the German Society for Digestive Diseases. He has published more than 350 peerreviewed papers.

Web of Science Citation Report (30.04.2013)

Results found: 839

Sum of the Times Cited: 18540

h-index: 61 


\section{How to Maximize Sorafenib Efficacy? \\ Peter R. Galle \\ Chairman, I. Dept. of Internal Medicine, University \\ Medical Center, Mainz, Germany}

The approval of sorafenib as the first effective drug for the treatment of hepatocellular carcinoma (HCC) has opened the field of systemic treatment of this disease. Sorafenib is currently the standard of care in advanced stage HCC (BCLC $\mathrm{C})$. However, as the improvement in survival is modest further treatment alternatives are clearly needed. Todays better understanding of HCC pathogenesis has led to the development of several novel targeted treatments. Unfortunately, by now we have already seen five phase III trials fail, demonstrating the need for further efforts and inovative trial design.

As Sorafenib is presently without alternative in systemic therapy of HCC it is of great importance to maximize it's efficacy. Several aspects can be taken into consideration: this includes patient selection, side effect management and the combination of Sorafenib with chemotherpy, other targeted agents and local ablative therapies. Concerning the latter it is conceivable to add Sorafenib in intermediate stage disease to TACE or to add TACE in advanced stage to Sorafenib.

It is mandatory that HCC is treated in a multidisciplinary way involving hepatologists, surgeons, interventional radiologists, and oncologists. In this setting patient selection and the respective choice of treatment according to stage are key. Sorafenib should be offered only to patients with well maintained liver function, in most cases not going beyond ChildPugh B7, as a functional liver reserve is required and hepatic decompensation can be a consequence of Sorafenib treatment.

Management of side effects. Sorafenib treatment is accompanied by adverse events including hand-foot-skin reactions which seems to be more frequent in the Asian population. Preventive measures, such as a pedicure before treatment, the use of shock absorbers to relieve painful pressure points, and frequent application of emollients that contain 5-10\% urea are recommended. When lesions are very painful, treatment should be interrupted for 1-2 weeks.

Combination with chemotherapy. The combination of Sorafenib with doxorubicin has been investigated in a phase II trial and shown to be feasible and more effecitive than doxorubicin alone. However, it remains to be determined if doxorubicin adds efficacy to Sorafenib alone.

Combination with other targeted therapies. The combination of agents targeting different pathways may be an option to overcome resistance to a single drug. A phase III trial (SEARCH) combining erlotinib inhibiting epidermal growth factor receptor tyrosine kinase with sorafenib failed to show a better response for combination therapy and was hampered by more side effects.

Combination with TACE. Several trials have assessed the combination of Sorafenib with TACE in intermediate and advanced stage HCC. The results are inconclusive due to heterogenous trial design; particularly randomized, controlled data are lacking. However, the combination of TACE with sorafenib appears to be safe and feasible in patients with intermediate (to advanced) stage HCC and toxicity profiles are similar to those of sorafenib and TACE alone in most cases. The combination appears promising and preliminary efficacy was shown; however, results of ongoing phase III RCTs are needed to clarify the clinical implication of this combined treatment approach in unresectable HCC (BCLC B and BCLC C).

Last but not least the experience of the physician plays a critical role to guide the patient through Sorafenib therapy and to avoid early treatment discontinuation. 


\section{Curriculum Vitae}

Name

Position

Institution

E-mail
Ho Yeong Lim

Professor

Samsung Medical Center Sungkyunkwan University hoylim@skku.edu

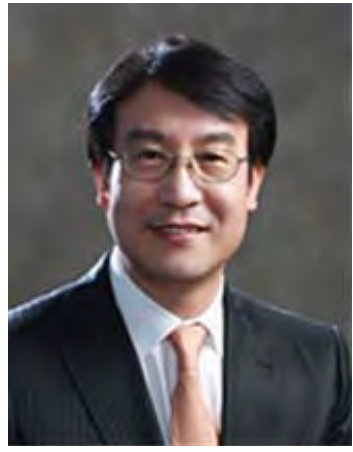

Ho Yeong Lim is a professor of Medical Oncology, Samsung Comprehensive Cancer Center, Samsung Medical Center, Sungkyunkwan University, School of Medicine, Seoul, Korea.

Professor Lim graduated from Yonsei University College of Medicine, Seoul, Korea and received MD in 1984. Professor Lim completed his internship and residency in internal medicine at Yonsei University Severance Hospital. He subsequently completed fellowship training in Medical Oncology at Yonsei Cancer Center in 1993. From 1993 to 2006, he worked as a professor of medical oncology at Ajou University, Suwon, Korea. Since 2006, he is a professor of medicine, Samsung Medical Center, Sungkyunkwan University, Seoul, Korea. He was appointed the chair of the Hepatobiliary and pancreas cancer Committee of the Korean Cancer Study Group from 2007 to 2013.

\section{Research Interest:}

Professor Lim's main interest of clinical and research work are clinical trials in gastrointestinal cancer including hepato-biliary cancer and basic research of hepatocarcinogenesis. He is an active member of many professional societies including Korean Association of Clinical Oncology, Korean Cancer Research Association and ASCO. 


\section{Second-line Treatment for Patients with Advanced Hepatocellular Carcinoma after Sorafenib Failure}

\author{
Ho Yeong Lim \\ Oncology, Samsung Comprehensive Cancer Center, \\ Samsung Medical Center, Sungkyunkwan University \\ School of Medicine, Seoul, Korea
}

Hepatocellular carcinoma (HCC) is one of the most common malignancies worldwide, and is highly prevalent in Asia. In Korea, hepatocellular carcinoma (HCC) accounts for approximately $10 \%$ of all malignancies and is one of the leading causes of cancer death.

Recent advances in understanding of the molecular mechanisms of hepatocarcinogenesis provide novel approach with targeted agents for the treatment of this lethal tumor. It has been recently recognized that several important intracellular signaling pathways and angiogenesis may have pivotal roles in the molecular pathogenesis of HCC. With increasing knowledge of the mechanisms of hepatocarcinogenesis and the success of molecular targeted agents in other cancers, there has been opened new horizon in the management of advanced HCC. Recently various molecular targeted agents have been evaluated in the treatment of advanced HCC, most of them block angiogenesis and cellular proliferation.

Sorafenib (Nexavar $\AA$, Bayer-Schering Pharma) is a multi-target tyrosine kinase inhibitor with effects on tumor proliferation and angiogenesis. Sorafenib is the first and so far the only drug that has shown survival advantage in HCC patients. In the Sorafenib HCC Assessment Randomized Protocol (SHARP) study and Asia-Pacific study, significantly longer progression-free survival (PFS) and overall survival (OS) were achieved with sorafenib compared with placebo. However, sorafenib provides short duration of disease stabilization, these two large randomized trials showed median time to progression of 5.5 months and 2.8 months with sorafenib, respectively. These results suggest the rapid emergence of resistance to this drug. Resistance to sorafenib may occur either through intrinsic or extrinsic mechanisms, however, our knowledge about the mechanisms of resistance is insufficient. For improving of treatment outcome with sorafenib and other promising drugs, it is important to understand the key oncogenic drivers and the resistance mechanisms to drugs in HCC.

Recently, the results of several novel agents after sorafenib failure have been published, and various agents are being studied as second-line therapy with different phases of clinical trials. Most of these novel agents are categorized to agents blocking angiogenesis or agents inhibiting cell growth signals.

The upregulation of proangiogenic pathways, such as fibroblast growth factor (FGF) pathway, can act as a resistance mechanism on sorafenib, a VEGFR inhibitor. Recent phase II trial of brivanib, an oral selective dual inhibitor of FGFR and VGFR tyrosine kinase, as second-line therapy showed a manageable safety profile and promising antitumor activity in 46 patients with advanced HCC. Randomized phase III trial of brivanib in patients with advanced HCC who failed or were intolerant to sorafenib (BRISK-PS) was completed and presented recently. There were improvements in TTP, DCR and ORR, indicating anti-tumor activity of brivanib, even though the primary endpoint of improved OS was not met, and brivanib had an acceptable safety profile.

c-MET was discovered as an oncogene and encodes a tyrosine kinase-type growth factor receptor with an affinity for hepatocyte growth factor (HGF). The binding of active HGF to functionally established c-MET leads to receptor dimerization/multimerization, multiple tyrosine residue phosphorylation in the intracellular region and downstream signaling for motility, proliferation, survival and morphogenesis. c-Met overexpression is observed in $20-48 \%$ of human HCC samples. Recently, a phase II trial of tivantinib (ARQ197), a c-Met inhibitor, in HCC patients was reported, and c-Met overexpression was proposed as a predictive biomarker.

JX-594 (Pexa-Vec) is a vaccinia virus with disruption of the viral thymidine kinase gene (TK) for cancer selectivity and insertion of human granulocyte-macrophage colony-stimulating factor (hGM-CSF) and $\beta$-galactosidase transgenes for immune stimulation and replication assessment, respectively. JX-594 is designed to selectively replicate within, and subsequently lyse, cancer cells while inducing tumor-specific immunity. Recent randomized dose-finding trial of JX-594 in liver cancer demonstrated oncolytic and immunotherapy MOA, tumor responses and dose-related survival in patients with HCC.

Currently, various novel agents are under investigation for the patients who failed prior sorafenib, unfortunately, no drug has shown salvage activity. There is no approved agent to date for HCC patients who progress on sorafenib. Effective treatment for HCC after sorafenib failure remains an urgent unmet medical need, therefore we need continuing research to identify key molecular targets and reliable biomarkers to overcome these challenges.

\section{References}

LLovet JM, Ricci S, Mazzaferro V et al. Sorafenib in advanced hepatocellular carcinoma. N Engl J Med 2008;359:378.

Cheng AL, Kang YK, Chen Z et al. Efficacy and safety of sorafenib in patients in the Asia-Pacific region with advanced hepatocellular carcinoma: a phase III randomized, double blind, placebocontrolled trial. Lancet Oncol 2009;10:25.

Berasain C. Hepatocellular carcinoma and sorafenib: too many resistance mechanisms?. Gut 2013 [E-pub].

Zhu AX. Molecularly targeted therapy for advanced hepatocellular carcinoma in 2012: current status and future perspectives. Semin Oncol 2012;39:493.

Porta C, Paglino C. Medical treatment of unresectable hepatocellular carcinoma: going beyond sorafenib. World J Hepatol 2010; 27:103.

Villanueva A, Llovet JM. Second-line therapies in hepatocellular carcinoma: emergence of resistance to sorafenib. Clin Cancer Res 2012;18(7):1824.

Finn RS, Kang Y-K, Mulcahy M, et al. Phase II, open-label study of brivanib as second-line therapy in patients with advanced hepatocellular carcinoma. Clin Cancer Res 2012;18(7):2090.

Santoro A, Rimassa L, Borbath I, et al. Tivantinib for second-line treatment of advanced hepatocellular carcinoma: a randomized, placebo-controlled ohase 2 study. Lancet Oncol 2013;14:55.

Heo J, Reid T, Ruo L, et al. Randomized dose-finding clinical trial of oncolytic immunotherapeutic vaccine JX-594 in liver cancer. Nat Med 2013;19(3):329. 


\section{Curriculum Vitae}

$\begin{array}{ll}\text { Name } & \begin{array}{l}\text { Masatoshi Kudo } \\ \text { Co-chairman of Organizing } \\ \text { Committee }\end{array} \\ \text { Institution } & \text { Kinki University } \\ \text { E-mail } & \text { m-kudo@med.kindai.ac.jp }\end{array}$

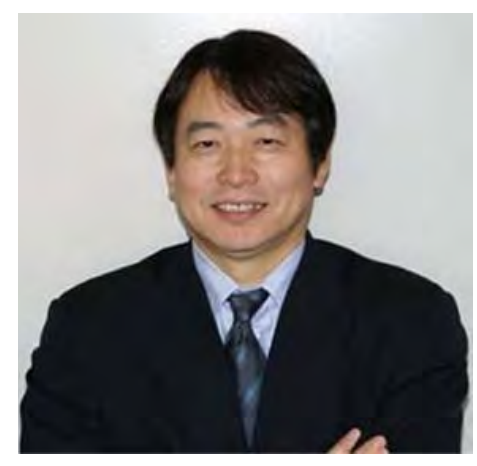

Masatoshi Kudo is currently Council of ILCA since 2009. He is a Professor and Chairman at the Department of Hepatology and Gastroenterology, Kinki University School of Medicine since 1999 and a President of Kinki University Medical Center since 2008.

Professor Kudo has published 433 International scientific peer review papers in well-regarded journals in addition to 959 domestic scientific papers. He serves as an Executive Council Board Member for Liver Cancer Study Group of Japan (LCSGJ), Chairman of Nationwide Survey Committee of LCSGJ, and a representative of LCSGJ Head Office. He is an Editor-in-Chief of the journal "LIVER CANCER". He is also serving as an Associate Editor or Editorial Board Member of numerous international journals.

He currently serves as a Secretary of Asian Federation of Societies for Ultrasound in Medicine and Biology (AFSUMB), and President of World Federation of Societies for Ultrasound in Medicine and Biology (WFUMB).

\section{Research Interest:}

Hepatology 


\section{Treatment Strategy for Early Stage of HCC \\ Masatoshi Kudo \\ Department of Gastroenterology and Hepatology, Kinki University School of Medicine, Osaka, Japan}

Japanese, European and American clinical practice guidelines strongly recommend surgical resection (SR) and percutaneous ablation, including radiofre-quency ablation (RFA) and percutaneous ethanol injection (PEI), for the management of early or moderately advanced HCC (i.e., up to 3 tumors $3 \mathrm{~cm}$ or less in diameter) in patients with adequately maintained liver function. Although comparative studies of these treatments have been conducted previously, the most suitable treatment strategy still remains controversial.

By nationwide surveys initiated in 1965, the Liver Cancer Study Group of Japan (LCSGJ) has prospectively collected data on patients with HCC in Japan.

Hasegawa Kand LCSGH reported that the overall survival rates at $3 / 5$ years were $85.3 \% / 71.1 \%$ in the SR group, $81.0 \% / 61.1 \%$ in the RFA group, and $78.9 \% / 56.3 \%$ in the PEI group. The median survival times were 8.4, 5.9, and 5.6 years in the three groups, respectively. The time to recurrence rates at $3 / 5$ years in the 3 groups were $43.3 \% / 63.8 \%, 57.2 \% / 71.7 \%$, and $64.3 \% / 76.9 \%$, respectively (Hasegawa $\mathrm{K}$ et al J Hepatol 2013).

These results indicated that the overall survival and time to recurrence rates were both significantly better in the SR group than in the RFA and PEI groups.

The results of the subgroup analyses showed that the overall survival was significantly longer in the SR group than in the RFA group in 2 subgroups of patients, namely, those who had a single tumor smaller than $2 \mathrm{~cm}$ in diameter with liver damage class $\mathrm{A}$, and those who had a single tumor $2 \mathrm{~cm}$ or larger in diameter with liver damage class $\mathrm{B}$.

In conclusion, LCSGJ nationwide study and current Japanese guideline in the management of HCC recommend RFA or resection for 2 or 3 nodules, less than $3 \mathrm{~cm}$ in diameter, however, resection is the first choice of treatment modality of patients with single tumor.

\section{How to evaluate treatment response in HCC: An mRESIST criterion enough?}

Masatoshi Kudo, Tadaaki Arizumi, Kazuomi Ueshima

Department of Gastroenterology and Hepatology, Kinki University Faculty of Medicine, Osaka, Japan

Hepatocellular carcinoma (HCC) is the third most common cause of cancer mortality worldwide, and a considerable number of patients continue to be diagnosed withadvanced disease. Recently, sorafenibhas been shown to improve the survival of patients with advanced-stage HCC; the effectiveness attributes to its unique antiproliferative and antiangiogenic mechanism.

In recognition thatthat the vascularity of a lesion is important in evaluating response to HCC treatment, the modified-RECIST (mRECIST) requires assessment oftumor vascularity, which reflects the extent of tumor necrisis. However, use of the mRECIST still poses difficulty in measuring irregularly shaped tumors because it calls forunidirectional measurement of tumor size for overall evaluation of tumor burden.

To test the hypothesis that use of the Response Evaluation Criteria in Cancer of the Liver (RECICL), an improved evaluation system designed to address the limitations of the Response Evaluation Criteria in Solid Tumors 1.1 (RECIST1.1) and modified RECIST (mRECIST), provides for more accurate evaluation of response of patients with hepatocellular carcinoma (HCC) to treatment with sorafenib, a molecularly targeted agent, as assessed by overall survival (OS).

The therapeutic response of 156 patients with advanced HCC who had been treated with sorafenib therapy for more than 1 month was evaluated using the RECIST1.1, mRECIST, and RECICL. After categorization as showing progressive disease (PD), stable disease (SD), or objective response, the association between OS and categorization was examined using the Kaplan-Meier methodto develop survival curves. The 141 cases categorized as PD or SDby the RECIST1.1 but objective response by themRECIST and RECICL were further analyzed for determination of the association between OS and categorization.

Only categorization using the RECICL was found to be significantly correlated with OS $(p=0.0033)$. Among the patientscategorized as SD or PD by the RECIST1.1, reclassificationby the RECICL but not the mRECIST was found to be significantly associated with OSand allow for precise prediction of prognosis $(p=0.0066)$.

Inconclusion, only the use of the RECICL allowed for identification of a subgroup of HCC patients treated with sorafenib with improved prognosis. The RECICL should therefore be considered a superior system for assessment of therapeutic response. 


\section{Curriculum Vitae}

$\begin{array}{ll}\text { Name } & \text { Joong-Won Park } \\ \text { Position } & \text { Principal Scientist } \\ \text { Institution } & \text { National Cancer Center, Korea } \\ \text { E-mail } & \text { jwpark@ncc.re.kr }\end{array}$

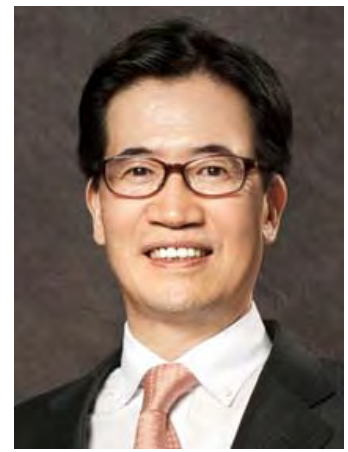

Joong-Won Park is a Principal Scientist of the National Cancer Center, Korea. He was the Head of the Center for Liver Cancer, NCC, Korea from 2002 to 2010 and Head of Translational and Clinical Research at the National Cancer Center Research Institute from 2009 to 2011. Dr Park studied Medicine at Seoul National University and graduated in 1984. He completed a residency in Internal Medicine and a Clinical Fellowship in Hepatology at Seoul National University Hospital, then completed a PhD in Medicine at Seoul National University in 1996. He was an Assistant and Associate Professor of Chung-Ang University Medical College from 1993 to 2002. He has also been a Visiting Scientist at the Center for Basic Research in Digestive Diseases, Mayo Clinic, Rochester, USA, from 1997 to 1999.

Dr. Park has published many International scientific papers in high-quality English-language journals as well as in local journals. He has also given many invited lectures on hepatitis and liver cancer at domestic and overseas meetings. He serves as Chair of the Committee for the Hepatocellular Carcinoma Management Guidelines of the Korea Liver Cancer Study Group (KLCSG)-NCC Korea. He is a member of the Korean Association for the Study of the Liver (KASL), the Asian-Pacific Association for the Study of the Liver (APASL), the American Association for the Study of Liver Diseases (AASLD), and the International Liver Cancer Association (ILCA). He serves as an Associate Editor of Hepatology International and is a regular reviewer for Hepatology, the Journal of Gastroenterology and Hepatology, and other international journals. He served as Chair of the Scientific Committee for the APASL 2008 Seoul meeting, a Chair of the Scientific Committee of the KASL from 2003 to 2005, and as Chair of the Scientific Committee of the KLCSG from 2002 to 2004.

\section{Research Interest:}

Dr. Park's research interests are the management of hepatocellular carcinoma, and hepatocarcinogenesis and molecularly targeted therapy. 
How to Manage HCC Patients with Incomplete or Refractory to TACE: Sorafenib

\author{
Joong-Won Park
}

National Cancer Center, Goyang, Korea

For patients with unresectable, large/multifocal hepatocellular carcinoma (HCC), transarterial chemoembolization (TACE) is recommended as a first-line treatment in most guidelines. While the Barcelona Clinic Liver Cancer (BCLC) guidelines and other guidelines suggested TACE to be the standard of care for intermediate stage HCC, there is considerable uncertainty when TACE should be stopped given the variable nature of clinical responses. This uncertainty of TACE refractoriness or failure not sufficiently addressed by existing guidelines includes the evaluation of treatment response, the criteria for repeating TACE and follow-up interval before switching to another treatment. Because TACE is a locoregional therapy, progression in the untreated area doses not necessarily imply the failure of TACE. The recent proposal of 'stage progression' which was defined as the development of vascular invasion or extrahepatic spread during follow up, may provide a surrogate end-point for TACE refractoriness. Taking this proposal into consideration along with Japanese guidelines, three sessions of TACE within 6 months should be adequate for effective tumor control. For those who have failed TACE (or refractory to TACE) with multifocal recurrence/remains or stage progression, sorafenib is recommended.

Because of the theoretical advantage of combining TACE with sorafenib, a number of trials are evaluating this combination in intermediate stage or in advanced stage of HCC patients. The combination of sorafenib in intermediate stage HCC or combination of TACE in advanced stage may improve the efficacy of TACE or sorafenib therapy, respectively. However, the successful data will be essential before recommending the combination therapy for patients with intermediate stage or advanced stage HCC. Several phase III controlled studies are ongoing in TACE-sorafenib combination therapy for patients with intermediate/advanced stage HCC and the outcomes of these trials are likely to change the recommendation for patients with HCC. 


\section{Curriculum Vitae}

\begin{tabular}{|c|c|}
\hline Name & Shuntaro Obi \\
\hline Position & $\begin{array}{l}\text { Director, The chair of } \\
\text { Hepatology, Kyoundo Hospital \& } \\
\text { Director, Sasaki Foundation }\end{array}$ \\
\hline Institution & $\begin{array}{l}\text { Department of Hepatology, } \\
\text { Kyoundo Hospital }\end{array}$ \\
\hline E-mail & obishun@gmail.com \\
\hline
\end{tabular}

I educated from Prof. Masao Omata at Graduate of Medicine, The University of Tokyo.

I have been performing TAE more than 1600 patients and Hepatic Arterial Infusion.

Chemotherapy more than 850 patients. Also I have been performing palliative care.

More than 100 patients / year die of hepatocellular carcinoma at our institute.

\section{Present Position or Academic Rank:}

2006-present Director, Kyoundo Hospital, Tokyo, Japan

2002-present The chair of Hepatology, Kyoundo Hospital

2005-present Director, Sasaki Foundation

2008-present Assistant Professor in Gastroenterology, Tokyo University School of Medicine

2009-present Assistant Professor in Gastroenterology Teikyo University School of Medicine

2011-present Assistant Professor in Gastroenterology Kagawa University School of Medicine

2012-present Assistant Professor in Gastroenterology Yamanasi University School of Medicine

Previous Professional Positions and Appointments:

1995-1998 Staff Physician, Internal Medicine (II), Tokyo University School of Medicine, Tokyo, Japan

1998-2002 Staff Physician, Gastroenterology, Tokyo University School of Medicine 


\section{Combination Therapy of Arterial Infusion 5-Fluorouracil and Systemic Interferon- Alpha for TACE Failure of HCC}

Shuntaro Obi, Shinpei Sato, Toshihiro Kawai, Takafumi Sugimoto, Youko Yashima, Miho Kanda

Kyoundo hospital of the Sasaki institute, Liver unit, Tokyo, Japan

Aim: Hepatic arterial infusion chemotherapy (HAIC) is major treatment modality for advanced hepatocellular carcinoma (HCC) in Japan. Prognosis of HAIC depends on response. The aim of this study is to clarify effectiveness of HAIC for TACE failure.

Methods: Eight hundred and sixty one patients with advanced HCC received Peg-IFN alpha 2a ( $90 \mu \mathrm{g}$ subcutaneous injection on Days 1 of each week of treatment) and 5-FU (500 mg into hepatic artery on Days 1-5 of the first and second week of each 4-week cycle). The therapy was either terminated at the end of the first cycle in cases with progressive disease, or continued for at least 2 cycles, when responses to treatment were evaluated by RICIST. The survival rate was calculated by Kaplan-Meier method. Predictive factors for survival were calculated by Cox proportional-hazards regression. Predictive factors for effectiveness were calculated by logistic regression.

Results: Of 861 patients, 106 patients (12\%) had extra hepatic metastasis and 586 patients (68\%) had vascular invasion. Remaining 169 patients were TACE failure. Of 169 patients, Child-Pugh A/B/C was 128/27/4. The median survival time (MST) was 11.1 months among overall patients. In Child-Pugh A, MST was 12 months. CR/PR/SD/PD was $10 / 38 / 13 / 68$. Response rate was $28 \%$. MST of the CR was 24 months, PR was 20 months, SD was 7 months and PD was 7 months. Predictive factors for survival were Platelet counts, T.Bil, Alb, ALT, and ascites. Predictive factors for effectiveness were Alb. Response rate was $37 \%$ in Alb $>3.5$ group, but $16 \%$ in $\mathrm{Alb}<3.5$ group.

Conclusion: In TACE failure, the combination therapy with 5-FU and IFN substantially improved the survival rate among the responders. 


\section{Curriculum Vitae}

Name

Position

Institution

E-mail
Hee Chul Park

Associate Professor

Samsung Medical Center,

Sungkyunkwan University

School of Medicine

rophc@skku.edu

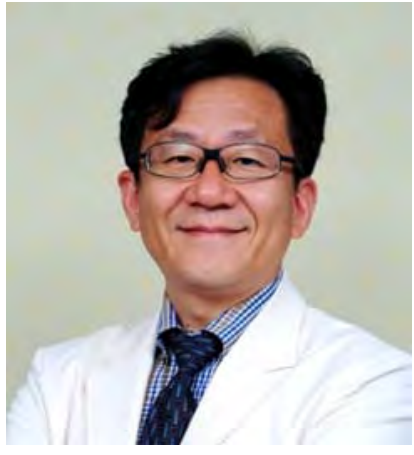

Dr. Hee Chul Park is currently an associate professor of Department of Radiation Oncology at Samsung Medical Center, Sungkyunkwan University School of Medicine, Seoul, Korea.

He graduated Yonsei University College of Medicine and went through the residency courses at Yonsei Cancer Center from 1996-1999. He performed clinical fellowship at Chosun University Hospital, Yonsei Cancer Center from 2000-2001. He earned his master's degree and doctoral dissertation at Yonsei University.

He published 49 articles in international journals and gave more than 50 invited talks on radiotherapy for hepatocellular carcinoma or on image guided precision radiotherapy.

Research Interest:

1. Clinical researches

- image-guided precision radiotherapy

- radiotherapy for hepatocellular carcinoma

- particle beam radiotherapy for hepatocellular carcinoma

2. Basic researches

- respiratory synchronized radiotherapy

- bio-effect radiotherapy planning

- radiation biology (tumor hypoxia, cancer stem cell, radioimmunotherapy) 


\section{How to Manage HCC Patients with Incomplete or Refractory to TACE?: External Radiotherapy}

Hee Chul Park

Samsung Medical Center, Sungkyunkwan University

School of Medicine, Seoul, Korea

TACE is not classified as a curative treatment, because complete tumor necrosis is not likely to be obtained in relatively large tumors despite repeated sessions of treatments. According to the results of a prospective cohort study from Korea, in which surgical resection after primary TACE was compared with TACE monotherapy, the overall survival rate was significantly higher for the surgical resection group as opposed to that of TACE monotherapy group. Combined treatment of TACE and other local ablative therapies may improve the local tumor response and survival outcomes. RFA or PEIT is a candidate local modality. Radiotherapy (RT) is believed to serve as local modality to enhance the local control of TACE.
With an increasing number of TACE procedures, incomplete TACE triggers tumor hypoxia, subsequently resulting in HCC either refractory to the treatment or facilitating intra- or extra-hepatic metastasis. For facilitated intra- or extra-hepatic metastasis from the refractoriness of repeated TACE, sorafenib could be a theoretical option to prevent or treat that form of disease progression. For failure to obtain local control after the repeated TACE, RT could be used as a local modality.

In other aspects, in cases where TACE is not feasible because of a severe arteriovenous shunt caused by a tumor, radiation therapy can cause vascular occlusion in approximately $20 \%$ of patients, thus making it possible to perform TACE.

There are two different ways of RT application to manage HCC patients with incomplete or refractory to TACE. For incomplete local control after initial TACE, RT could be used as a "consolidation" planned procedure to target cancer cells at the tumor periphery that may remain viable due to blood supply from the collateral circulation or recanalization of the embolized artery. After the failure to obtain local control in spite of repeated TACE, RT could be used as a "salvage" treatment for tumors unresponsive to TACE. 
The 4th Asia-Pacific Primary Liver Cancer Expert Meeting

\section{Curriculum Vitae}

$\begin{array}{ll}\text { Name } & \text { Andrew X. Zhu } \\ \text { Position } & \begin{array}{l}\text { Director, Liver Cancer Research } \\ \text { Associate Professor of Medicine }\end{array} \\ \text { Institution } & \begin{array}{l}\text { Massachusetts General Hospital } \\ \text { Cancer Center, Harvard Medical }\end{array} \\ & \text { School } \\ \text { E-mail } & \text { azhu@partners.org }\end{array}$

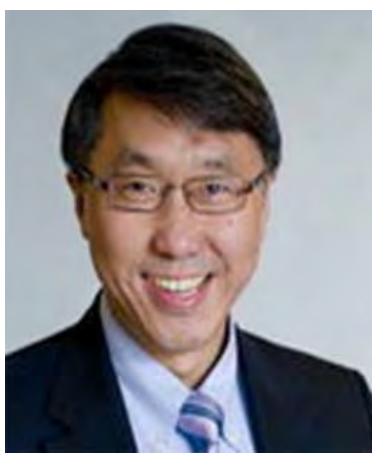

Dr. Andrew X. Zhu is Director of Liver Cancer Research at Massachusetts General Hospital Cancer Center and an Associate Professor of Medicine at Harvard Medical School. As a widely published author, Dr. Zhu has served as a principle investigator in many clinical trials in HCC, BTCs and other gastrointestinal cancers. He is the invited reviewer for many medical journals and has lectured extensively on HCC and other gastrointestinal cancers. An internationally recognized leader in HCC, he has led early efforts of developing several molecularly targeted agents in HCC and studying the predictive and surrogate circulating and imaging biomarkers. Dr. Zhu is the principle investigator of three phase III trials in advanced HCC. He is a founding board member of the International Liver Cancer Association, Vice President of CanLiv-The Hepatobiliary Cancers Foundation, Fellow of American College of Physicians, and a member of the American Society of Clinical Oncology (ASCO) and the American Association for Cancer Research. Dr. Zhu serves on the Hepatobiliary Cancer committee of the National Comprehensive Cancer Network, the Grants Selection Committee of ASCO, and the Hepatobiliary Cancer Task Force of The NCI Gastrointestinal Cancer Steering Committee (GISC).

\section{Research Interest:}

1 To develop more effective therapies for hepatocellular carcinoma (HCC) and biliary tract cancers (BTCs) through phase I, II and III clinical trials.

2 To develop novel circulating and imaging biomarkers for targeted therapeutics that have prognostic and/or predictive significance.

3 To define and characterize known or novel genetic mutations in HCC and BTCs and assess their potential correlation with clinical outcomes and as therapeutic targets. 


\section{Molecular Targeted Therapy in HCC: Current Status and Future Direction}

Andrew X. Zhu

Massachusetts General Hospital Cancer Center, Harvard Medical School, Boston, MA, USA

Worldwide, hepatocellular carcinoma (HCC) is the sixth most common cancer and the third most common cause of cancer-related death. The incidence rates for HCC in the United States and Europe have been rising. Advanced HCC carries a poor prognosis and systemic therapy with cytotoxic agents provides marginal benefit. Improved understanding of the mechanism of hepatocarcinogenesis coupled with the arrival of many newly developed molecularly targeted agents has provided the unique opportunity to study some of these novel agents in advanced HCC. Despite the successful approval and extensive application of sorafenib, the prognosis for patients with advanced HCC remains poor and the benefits with sorafenib are modest. In the past few years, there have been renewed and continued interests and active research in developing other molecularly targeted agents in HCC. While the initial efforts are focusing on anti-angiogenic therapy, other agents targeting the epidermal growth factor-receptor, mammalian target of rapamycin (mTOR), hepatocyte growth factor/c-Met among others have entered HCC clinical trials. Combining different molecularly targeted agents or combining targeted agents with chemotherapy represent other strategies under investigation. The author will attempt to summarize the current status of other molecularly targeted agents or regimens beyond sorafenib under development in advanced HCC and discuss the future perspectives. 


\section{Curriculum Vitae}

$\begin{array}{ll}\text { Name } & \text { Pierce K.H. Chow } \\ \text { Position } & \begin{array}{l}\text { Senior Consultant Surgeon and } \\ \text { Senior Clinician Investigator }\end{array} \\ \text { Institution } & \begin{array}{l}\text { Singapore General Hospital } \\ \text { pierce.chow.k.h@sgh.com.sg }\end{array}\end{array}$

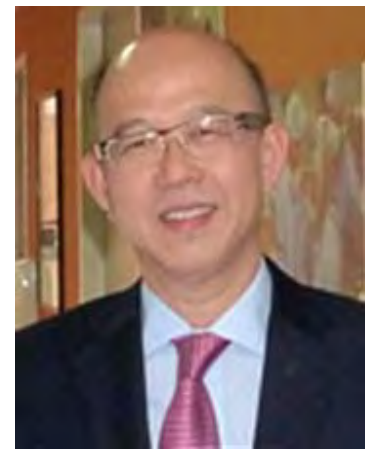

Pierce Chow is Professor at the Duke-NUS Graduate Medical School and Senior Consultant Surgeon at the SingHealth Group which includes the Singapore General Hospital (SGH) and the National Cancer Center Singapore. Pierce Chow co-founded the Asia-Pacific Hepatocellular Carcinoma (AHCC) Trials Group in 1997 and has been protocol chair of 5 multi-center trials that has involved 30 centers in 14 Asia-Pacific countries. He currently leads a phase III investigator initiated trial comparing sorafenib with a brachytherapy device SirSphere ${ }^{\circledR}$ for locally advanced liver cancer. He was conferred the 2012 NMRC National Outstanding Clinician Scientist for his research on Liver Cancer.

\section{Research Interest:}

Pierce's interests are in HPB surgery and translational research, clinical trials and health services outcomes research in oncology. Specific areas include: personalized medicine, clinical trials and experimental oncology in hepatocellular carcinoma, pancreatic carcinoma, gastro-intestinal stromal tumor, neuroendocrine tumor. Development of biomedical devices in oncology Meta-analysis, systemic review and health services outcomes research in hepatocellular carcinoma. Brachytherapy and Selective Internal Radiation Therapy in hepatocellular carcinoma. Patient tumour-explant xenografts and orthotopic models in experimental oncology. 


\section{Controversies for Locally Advanced HCC: How to Manage Advanced HCC with PVT?}

\author{
Pierce K.H. Chow
}

National Cancer Center Singapore, Duke-NUS Graduate Medical School, Singapore

Vascular invasion including portal vein invasion thrombosis/invasion (PVT) is one of the most devastating features of Hepatocellular carcinoma (HCC). PVT is a harbinger of grave prognosis and is rapidly fatally. If not successfully treated, the survival of HCC with PVT among Hep B patients in the Asia-Pacific is in the region of 3- 6 months. When the main portal vein is uninvolved, APASL guidelines allow surgical resection to prolong survival although surgery is contra-indicated under AASLD guidelines. TACE, a common modality of treatment in locally advanced HCC is contra-indicated in PVT. There is data however to show that radiation therapy, both brachytherapy with yttrium-90 and external beam radiation are useful in PVT as is sorafenib. The controversies are discussed through a few case studies. 
The 4th Asia-Pacific Primary Liver Cancer Expert Meeting

\section{Curriculum Vitae}

$\begin{array}{ll}\text { Name } & \text { Ann-Lii Cheng } \\ \text { Position } & \text { Professor \& Director } \\ \text { Institution } & \text { National Taiwan University } \\ & \begin{array}{l}\text { Hospital } \\ \text { alcheng@ntu.edu.tw }\end{array}\end{array}$

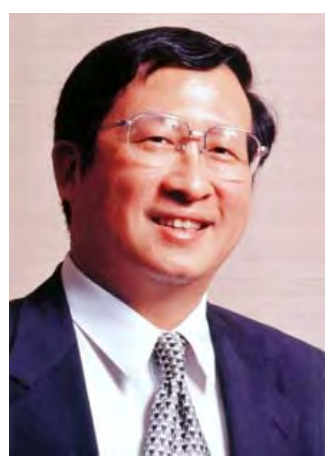

Ann-Lii Cheng is Professor and Chairman of the Department of Oncology of the National Taiwan University Hospital in Taipei, Taiwan. He received his MD degree, PhD degree, and his specialty training in Medical Oncology at the Medical School of the National Taiwan University. In 1990, he was a research fellow at the Comprehensive Cancer Center of the University of Wisconsin, Madison, USA. Dr. Cheng has been actively involved in basic and translational research in GI oncology, particularly hepatocellular carcinoma and gastric lymphoma, and has published more than 200 peer-reviewed articles. He received the "Outstanding Research Award" from the National Science Council of Taiwan, and the "Outstanding Cancer Research Award" from the Chinese Oncology Society. He became "Distinguished Professor" of National Taiwan University in 2006, and was elected as Fellow of American Association for the Advancement of Science (AAAS) in 2007. Dr. Cheng is an active member of the American Association for Cancer Research (AACR) and the American Society of Clinical Oncology (ASCO). He has served on the editorial boards of Oncology (Basel), Asia-Pacific Journal of Clinical Oncology, and Targeted Oncology - Biotherapies for the Clinicians in Oncology. In addition, Dr. Cheng is Chairman of the Chemotherapy Committee of the Taiwan Cooperative Oncology Group, of which he was one of the founding members. He received the national award of "outstanding contributions for science and technology" in 2008, and a national award for academic excellence in 2010. He served as president of the Taiwan Oncology Society during 20092011. He was elected as council member of International Liver Cancer Association (ICLA) in 2012.

\section{Research Interest:}

Translational research for hepatocellular carcinoma 


\section{Current Challenges to Manage HCC}

Ann-Lii Cheng

National Taiwan University Hospital, Taipei, Taiwan

Since sorafenib was approved in 2007, the field has been constantly challegnged by the repeated failures of new drug development in HCC. The mainstay of current development remains on anti-VEGFR muti-targeted TKI. Among these, drugs with additional activity on either FGFR or c-Met are considered most promising. Novel categories of molecular targeted agents (MTA), either alone or in combination with sorafenib, are in general at early stages of development.
Recent development of whole-genome sequencing has unveiled several novel genetic alterations which may have biologic significance. However, a true driver mutation, which is the key to success of MTA, remains elusive at this moment.

It is apparent that treatment modalities beyond MTA should be pursued. Along this line, oncolytic virotherapy, immunotherapy, and radiotherapy are on the horizon for a possibel breakthrough in this field. 


\section{Curriculum Vitae}

$\begin{array}{ll}\text { Name } & \text { Jeong Heo } \\ \text { Institution } & \text { Pusan National University Hospital } \\ \text { E-mail } & \text { jheo@pusan.ac.kr }\end{array}$

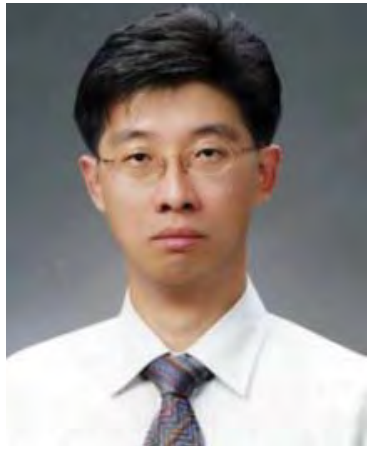

\section{Education:}

1987-1993, M.D.: Pusan National University College of Medicine, Pusan, Korea 1994-1996, M.S.: Postgraduate School, Pusan National University, Pusan, Korea 2001-2004, Ph.D.: Postgraduate School, Pusan National University, Pusan, Korea

\section{Professional Training and Employment:}

Mar.1993-Feb.1998

Internship and Residency, Internal Medicine, Pusan National University Hospital, Pusan, Korea

Mar.1998-Apr. 2001

Armydoctor, Ministry of National Defense (Rank: Captain)

May 2001-Feb. 2003

Clinical Instructor, Division of Gastroenterology and Hepatology,

Department of Internal Medicine, Pusan National University Hospital

Mar. 2003-Feb. 2007

Assistant Professor, Department of Medicine, Pusan National University, Pusan, Korea

Mar. 2008-Feb. 2013

Associate Professor, Department of Medicine, Pusan National University, Pusan, Korea

Mar. 2013-Present

Professor, Department of Medicine, Pusan National University, Pusan, Korea 


\section{Oncoviral therapy in advanced HCC Jeong Heo}

Department of Internal Medicine, Pusan National University School of Medicine, Pusan, Korea

JX-594 (Pexa-Vec) is a targeted oncolytic vaccinia virus designed to selectively replicate in and destroy cancer cells with epidermal growth factor receptor (EGFR)/ ras pathway activation. Direct oncolysis plus granulocyte macrophagecolony stimulating factor (GM-CSF) expression is accompanied by tumor vascular shutdown and anti-tumoral immunity. JX-594 injection into hepatocellular carcinoma (HCC) was well-tolerated and associated with viral replication, anti-cancer immunity, decreased tumor perfusion and tumor necrosis. JX-594 has been shown to be well-tolerated by intravenous (IV) infusion and intratumoral (IT) injection and JX-594 is being developed as a novel therapy for patients with refractory or advanced HCC. Synergistic anti-tumor effects are predicted with JX-594 and sorafenib due to acute vascular shut down effect and tumor-specific antibody formation of JX-594 and chronic anti-angiogenic effects of sorafenib. JX-594 shows promise as a novel agent for the treatment of advanced HCC.

\section{Reference}

1 Kim MK, Breitbach CJ, Moon A, Heo J, Lee YK, Cho M, Lee JW, Kim SG, Kang DH, Bell JC, Park BH, Kirn DH, Hwang TH. Oncolytic and immunotherapeutic vaccinia induces antibodymediated complement-dependent cancer cell lysis in humans. Sci Transl Med. 2013 May 15;5(185):185ra63. doi: 10.1126/scitranslmed.3005361.

2 Heo J, Reid T, Ruo L, Breitbach CJ, Rose S, Bloomston M, et al. Randomized dose-finding clinical trial of oncolytic immunotherapeutic vaccinia JX-594 in liver cancer. Nat Med. 2013 Feb 10;19(3):329-36. doi: 10.1038/nm.3089.

3 Breitbach CJ, Arulanandam R, De Silva N, Thorne SH, Patt R, Daneshmand $\mathrm{M}$, et al. Oncolytic vaccinia virus disrupts tumor-associated vasculature in humans. Cancer Res. 2013 Feb 15;73(4):1265-75. doi: 10.1158/0008-5472.CAN-122687.

4 Heo J, Breitbach CJ, Moon A, Kim CW, Patt R, Kim MK, et al. Sequential Therapy With JX-594, A Targeted Oncolytic Poxvirus, Followed by Sorafenib in Hepatocellular Carcinoma: Preclinical and Clinical Demonstration of Combination Efficacy. Mol Ther. 2011;19:1170-1179.

5 Parato KA, Senger D, Forsyth PA, Bell JC. Recent progress in the battle between oncolytic viruses and tumours. Nat Rev Cancer. 2005;5:965-976.

6 Kirn D, Martuza RL, Zwiebel J. Replication-selective virotherapy for cancer: Biological principles, risk management and future directions. Nat Med. 2001;7:781-787. 


\section{Curriculum Vitae}

$\begin{array}{ll}\text { Name } & \text { Jung-Hwan Yoon } \\ \text { Position } & \text { Professor } \\ \text { Institution } & \begin{array}{l}\text { Department of Internal Medicine } \\ \text { and Liver Research Institute, } \\ \text { Seoul National University College } \\ \end{array} \\ & \text { of Medicine } \\ \text { E-mail } & \text { yoonjh@snu.ac.kr }\end{array}$

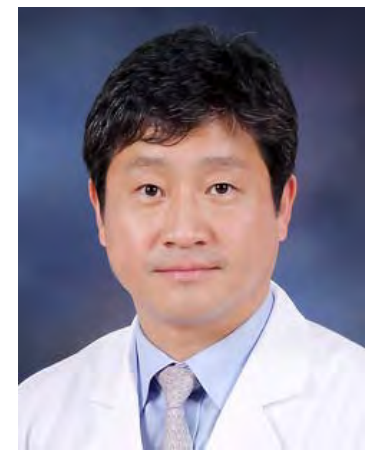

Professor Jung-Hwan Yoon graduated from Seoul National University College of Medicine (Seoul, Korea) in 1988. He got a masters and a doctor's degree of medicine from Seoul National University College of Medicine in 1992 and 1997, respectively. After he accomplished residency (from 1989 to 1992) and fellowship (from 1995 to 1997) at Seoul National University Hospital (SNUH; Seoul, Korea), he has been a faculty member of Seoul National University College of Medicine. He also conducted basic research at Mayo Clinic (Rochester, MN, USA) from 2001 to 2003 as a research fellow. Now he is a professor of medicine and the chairman of the Liver Research Institute at Seoul National University, and the Executive Director of Liver Cancer Center at SNUH. He served as the Scientific Director of Korean Association for the Study of the Liver (2010-2012) and is now serving as the Associate Editor of Digestive Diseases and Sciences. He has published approximately 110 international scientific papers and about 20 chapters of hepatology textbooks.

\section{Research Interest:}

Professor Yoon's research interests embraced both basic and clinical issues of hepatology.

His major basic research interests include: (i) the development of anticancer drugs inducing apoptosis of hepatocellular carcinoma (HCC) cells, (ii) the establishment of human HCC cell lines isolated from rapidly growing hypovascular HCCs, (iii) the development and application of novel targeted therapy for HCC, (iv) the improvement of the efficacy of adjuvant immune therapy to prevent HCC recurrence following curative treatments, (v) the differentiation of peripheral blood stem cells into hepatocytes, and (vi) the signals in hepatic fibrogenesis and their modification.

He is also actively conducting a number of (i) clinical trials employing locoregional and systemic therapies for HCC, and anti-viral treatment for chronic hepatitis $\mathrm{B}$ and $\mathrm{C}$, (ii) prospective cohort study to explore biomarkers and prognostic factors of HCC using up-to-date technologies and (iii) studies on mathematical modeling of various liver diseases. 


\section{Immunotherapy for HCC}

Jung-Hwan Yoon

Department of Internal Medicine and Liver Research Institute, Seoul National University College of Medicine, Seoul, Republic of Korea

Current standard therapeutic modalities for advanced hepatocellular carcinoma (HCC) are marginally effective and may worsen underlying liver disease. There is an unmet need for cutting edge, alternative therapies for HCC. Immunotherapy is principally suitable for HCC owing to its nontoxic, systemic and long-lasting anti-tumor activity. Strategies targeting tumor-associated antigens or viral antigens can induce activation of an HCC-specific response. In addition, whole tumor cell or tumor lysate-based immunization strategies can target mutated and uncharacterized antigens.
Several strategies including adoptive transfer of autologous activated T lymphocytes, cytokine injections, and autologous tumor-pulsed dendritic cells have been tested in human clinical trials. While therapeutic benefits have been achieved in early clinical trials, immunotherapy may be insufficient in patients with considerable disease burdens, and therefore, hepatic micro-metastases may be suitable targets. In addition, the efficacy of immune-based therapies is limited by several distinctive properties of HCC, most notably the inherently tolerance-inducing character of hepatocarcinogenesis including myeloid-derived suppressor cells. Therapeutic regimens that both amplify tumor-specific immunity and counteract these immunosuppressive mechanisms are expected to strongly improve clinical outcomes of HCC patients. 


\section{Curriculum Vitae}

$\begin{array}{ll}\text { Name } & \text { Jinsil Seong } \\ \text { Position } & \text { Professor in Radiation Oncology } \\ \text { Institution } & \text { Yonsei University Medical college } \\ \text { E-mail } & \text { jsseong@yuhs.ac }\end{array}$

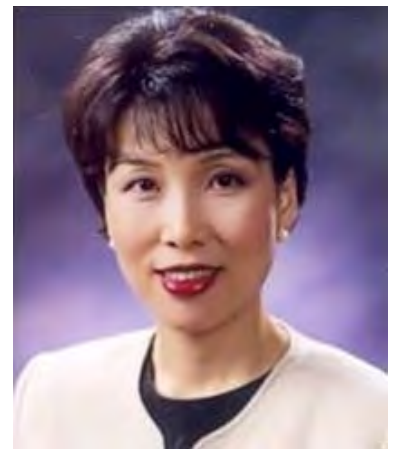

Dr Jinsil Seong currently works at Department of Radiation Oncology, Yonsei University Health System, Seoul. Prior to her current position, she has been a Visiting Scientist in Department of Experimental Radiotherapy, M.D. Anderson Cancer Center, Houston, USA. She graduated from Yonsei University College of Medicine, Seoul and subsequently completed her PhD degree from Yonsei University, Seoul.

Dr. Seong is a former President of Yonsei Liver Cancer Study Group and also a member of various national and international societies including International Liver Cancer Association, Korean Liver Cancer Study Group, European Society for Therapeutic Radiology and Oncology and American Society for Therapeutic Radiology and Oncology. Dr Seong is also a member of the Editorial Board of International Journal of Radiation Oncology Biology Physics, council of Asian Clinical Oncology Society as well as Asia Pacific Primary Liver Cancer Expert Meeting. She has been awarded many times for her credit including Korean Cancer Association Distinguished Scientific Award (2012), Best Presentation Award (bronze) in $2^{\text {nd }}$ Asia Pacific Primary Liver Cancer Expert Meeting (2011) and Young Investigator Award in International Congress of Liver Disease (2004). She has published 93 articles in non-SCI listed journals and 88 articles in SCI listed journals. She has also delivered 95 talks at major national and international congresses.

\section{Research Interest:}

"Radiotherapy of HCC" in clinical approach as well as in translational research. 


\section{Innovative Targeted Radiotherapy for Hepatocellular Carcinoma}

\section{Jinsil Seong}

Radiation Oncology, Yonsei University Medical College, Seoul, Korea

During the past 2 decades, advancement of computer technology including development of software and imaging has brought a new era in radiation oncology. As a result, intensity modulated radiotherapy (IMRT) and image guided radiotherapy have now entered into daily radiotherapy practice in clinic. Using simultaneous integrated boost IMRT (SIB-IMRT), precise and accurate delivery of high dose radiation can be possible in certain area inside the tumor. Certainly this approach has been successful by improving therapeutic ratio in cancers that dose-response relationship was proven.

However, limited success has been shown in most cancers, which can be attributed to radioresistance of tumor. Radioresistance can be a common characteristic of certain types of cancer but more frequently, shown as subvolume feature even in radiosensitive tumor. To overcome radioresistance of tumor, a sophisticated technology of innovative targeted radiotherapy is needed.
Targeted radiotherapy involves 2 fields of research; one is how to identify targetable subvolume (biology-based) and the other is how to hit it (physics-based). Physics-based targeting seems easy to approach since technology has been developed involving subvolume dose boosting and dose painting and already applied in clinical radiotherapy machine. Biology-based targeting, however, is more or less limited in real world due to the gap between research interest and practical need. Radiobiological studies have shown that radioresistance is related with hypoxia, proliferative potential, as well as proportion of cancer stem cells. Fortunately these biological processes can be identified through molecular imaging technology using appropriate radiotracers.

In radiotherapy of hepatocellular carcinoma, particularly locally advanced one, high dose of radiotherapy cannot be given due to toxicity. Consequently, there comes soon treatment failure. If we can identify resistant subvolume of tumor (biological targeting) and subsequently perform targeted delivery of focal high dose of radiation (physical targeting), therapeutic success can be much improved. Innovative targeted radiotherapy comes from combining biological as well as physical targeting technology, which will soon be applicable in the near future. 


\section{Curriculum Vitae}

$\begin{array}{ll}\text { Name } & \text { Richard S. Finn } \\ \text { Position } & \begin{array}{l}\text { Associate Professor of Medicine } \\ \text { Division of Hematology/ } \\ \text { Oncology }\end{array} \\ \text { Institution } & \begin{array}{l}\text { Geffen School of Medicine } \\ \text { at UCLA } \\ \text { rfinn@mednet.ucla.edu }\end{array}\end{array}$

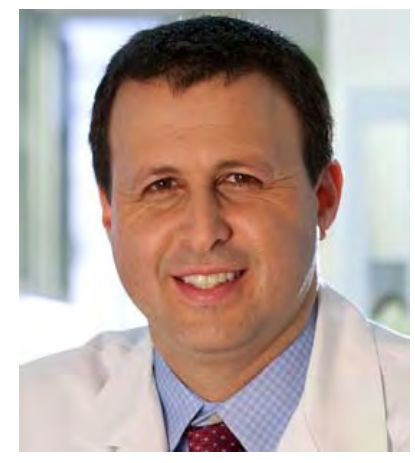

Dr. Richard Finn is an Associate Professor of Medicine in the Division of Hematology/ Oncology at the Geffen School of Medicine at UCLA. He is also Director of the Translational Oncology Research Laboratory and co-Director of the Signal Transduction and Therapeutics Program at the Jonsson Comprehensive Cancer Center. Dr Finn splits his time between patient care and research activities. In his 10 years on faculty, Dr. Finn has become the lead oncologist in multi-disciplinary liver cancer center at UCLA. He has been lead investigator in multiple clinical trials in HCC and is focused on bringing new approaches to treating liver cancer. Specifically, identifying new targets for therapy and designing clinical trials aimed at validating these approaches. He currently serves on the executive board of the International Liver Cancer Association (ILCA) and is on the editorial board of Clinical Cancer Research.

\section{Research Interest:}

Dr. Finn's research interests focus on bringing novel molecular targeted therapies from the laboratory to the clinic in HCC and breast cancer. 


\section{"Ongoing Trials in HCC"}

\section{Richard S. Finn}

Geffen School of Medicine at UCLA, Los Angeles, CA, USA

While the results of the two Phase III studies of sorafenib for advanced HCC represented a milestone in the treatment of the disease, the results of Phase III studies since that time have been disappointing. Despite great efforts, no new agents have been approved in the front-line or second line setting. Since the last APPLE meeting we have seen several negative Phase III studies which raises the question "What are we doing wrong?" In this lecture we will review the negative data behind the recent failures and highlight ongoing studies of new agents in HCC. This year we expect data Phase III data from studies for second-line HCC with the mTOR inhibitor everolimus and the VEGF receptor antibody, ramucirumab. Meanwhile, several new Phase III studies are being launched this year with the c-MET inhibitor tivantinib, the multi-kinase inhibitors reografenib and lenvatinib (E7080) and other agents. There are numerous other agents in Phase II and earlier studies including new inhibitors of the FGFR and VEGF axis, oncolytic viruses, and other novel approaches. In addition, while the incorporation of systemic agents with locally ablative treatments has carried much hope, to date they have yielded generally negative data. Critical to the successful development of new therapies will be a better understanding of the natural history of HCC after sorafenib as well as the incorporation of predictive markers of response to these prospective studies of these new drugs. 


\section{Curriculum Vitae}

$\begin{array}{ll}\text { Name } & \text { Byung Ihn Choi } \\ \text { Position } & \text { Professor } \\ \text { Institution } & \text { Seoul National University Hospital } \\ \text { E-mail } & \text { bichoi@snu.ac.kr }\end{array}$

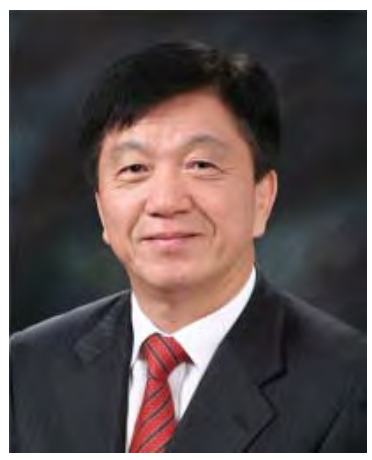

Dr. Choi is a professor of radiology at Seoul National University, College of Medicine. He is a Past chair of the Department of Radiology at the Seoul National University Hospital, and is also a former president of 5 national and international societies including The Korean Society of Radiology (KSR), Asian Oceanian Society of Radiology (AOSR), the Asian Society of Abdominal Radiology (ASAR) and Asian Federation of Societies of Ultrasound in Medicine and Biology (AFSUMB), and member of executive committee of 7 International organizations of Radiology including International Society of Radiology (ISR). He is an honorary member of 10 international and regional societies including RSNA and ESR. He is also an honorary fellow of 3 prestigious college of radiology including American College of Radiology (ACR). Dr Choi is a world renowned abdominal radiologist, particularly in the field of hepatobiliary imaging. He is a tireless and prolific clinical and scientific researcher, devoted teacher, and an outstanding clinician. Dr. Choi has authored more than 360 scientific papers and 28 textbooks, and presented more than 320 lectures internationally.

\section{Research Interest:}

1 Evaluation of abdominal imaging techniques inducing Ultrasound, CT, MRI and fusion imaging.

2 Development of new abdominal radiology procedures.

3 Development for early detection of abdominal cancer including liver, biliary tree, pancreas and gastrointestinal tract.

4 Evaluation of quantitative diagnosis of abdominal cancer by functional imaging technologies.

5 Mentoring fellows and residents in abdominal radiology research. 


\section{New Paradigm for Management of HCC by Imaging \\ Byung Ihn Choi \\ Seoul National University, College of Medicine, Seoul, Korea}

Hepatocellular carcinoma (HCC) is the most common in eastern Asia and Sub-Saharan Africa. But, even in USA, HCC shows rising incidence from 1970s to present, and fastest growing death rate. Therefore, HCC is really a global problem. HCC is the $6^{\text {th }}$ most common malignancy worldwide, $6 \%$ of all cancer. HCC is still a devastating cancer with 5year survival less than $5 \%$.

There are many clinical practice guidelines for HCC worldwide including Europe, Korea, America, Japan and Asia and pacific region. According to those guidelines, dependency of imaging technique is increasing for the diagnosis of HCC, and importance of biopsy is decreasing.

Since the 2000 EASL guideline has been announced, all guidelines in the world show increase of use of dynamic US/ CT/MRI with confirmative diagnostic criteria including hypervascular nature in arterial phase and washout in delayed phase image and decrease of use of biopsy and $\alpha \mathrm{FP}$, and decrease of size criteria.

A variety imaging modalities, including ultrasound (US), computed tomography (CT), magnetic resonance imaging (MRI), nuclear medicine, and angiography, are currently used in evaluating patients with chronic liver disease and suspected HCC. Further technological advancement will undoubtedly have a major impact on HCC imaging. Increased speed of data acquisition and consequently shorter scan times in CT and MRI show further improvement in resolution by further reducing motion artifacts. Development of new contrast materials for HCC imaging in US and MRI improves tumor detection and characterization by increasing the contrast and temporal resolution.

Currently available advanced US techniques in the evaluation of HCC are various harmonic imaging techniques with contrast agents, volume imaging, and recently, US elastography, that has been developing and might play a role in characterizing liver nodules in the future. The latest advance in CT is the multidetector (MD) CT scanner where 320-detector CT was introduced. Recent studies describe the high sensitivity of arterial phase imaging in hepatic tumor detection and the usefulness of CT angiography by using MD CT in a detailed assessment of hepatic arterial anatomy using a three-dimensional dataset. In addition, per-fusion CT imaging is also being developed and can be used for the characterization and treatment monitoring of HCC. Dual-energy CT with new technology is also continuously progressing. Advances in MR technology, including hardware and pulse sequence implementation, allow acquisition times to be reduced to the time frame of one breath hold, providing multiphasic dynamic MRI. Functional MRI including diffusion-weighted MRI, MR elastography, and new MR contrast agent with dual function have been investigated for the clinical utility of detection and characterization of HCCs.

For effective treatment of HCC, early diagnosis is necessary. For that purpose, understanding of hepatocarcinogenesis is essential. Recently contrast-enhanced US, MDCT and MRI have reached a high standard for the detection and characterization of HCC with the possibility of multiphasic examinations, high-resolution imaging and functional capabilities. Therefore, they should be regarded as valuable noninvasive imaging modalities for detection and characterization of nodules in patients with liver cirrhosis.

However, it should be recognized that there is a diagnostic dilemma of small $(<1 \mathrm{~cm})$ arterial enhancing lesions with all imaging modalities. Although hemodynamic features of nodules in the cirrhotic liver remains as the main diagnostic criterion, newly developed cell specific MR contrast agents have shown a high possibility of improvement in detection and characterization of HCC, and might overcome the diagnostic dilemma for small lesions or borderline hepatocellular lesions.

New imaging and treatment techniques are continuously being developed and guidelines should be updated every 3 or 4 years, incorporating new evidence.

In 20th century paradigm of medical imaging, radiologic diagnosis was based on a morphological imaging and gross pathology correlation, but in the 21st century, modern medicine including pathology is rapidly changing from conventional morphologic medicine to molecular medicine on the basis of molecular pathology and biology. Therefore, we need a paradigm shift from structural imaging to biomedical, physiologic, functional and genetic imaging.

Fortunately, new concept, radiogenomics which is correlation of in-vivo radio-phenotype by radiologic imaging and molecular phenotype by in vitro molecular diagnostics, was introduced. In addition, multiparametric imaging is under investigation as the emerging field of radiology in 21st century. Therefore, multidisciplinary team approach including hepatologist, radiologist, surgeon, pathologist, biologist, chemist, physicist, and bioinformatics is necessary to improve the efficiency and accuracy of patient care with HCCs dramatically during screening, diagnosis, treatment and monitoring. 


\section{Curriculum Vitae}

\begin{tabular}{|c|c|}
\hline Name & Masatoshi Kudo \\
\hline Position & $\begin{array}{l}\text { Co-chairman of Organizing } \\
\text { Committee }\end{array}$ \\
\hline Institution & Kinki University \\
\hline E-ma & m-kudo@med.kindai.ac.jp \\
\hline
\end{tabular}

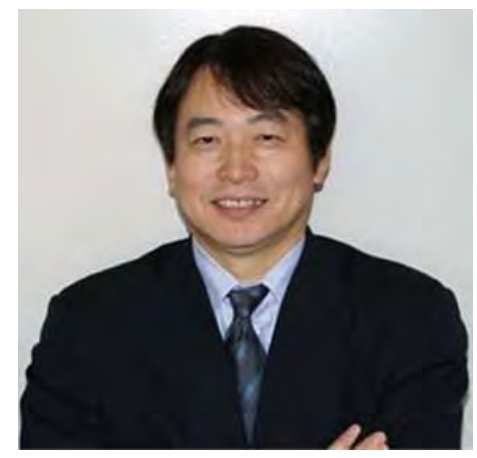

Masatoshi Kudo is currently Council of ILCA since 2009. He is a Professor and Chairman at the Department of Hepatology and Gastroenterology, Kinki University School of Medicine since 1999 and a President of Kinki University Medical Center since 2008.

Professor Kudo has published 433 International scientific peer review papers in well-regarded journals in addition to 959 domestic scientific papers. He serves as an Executive Council Board Member for Liver Cancer Study Group of Japan (LCSGJ), Chairman of Nationwide Survey Committee of LCSGJ, and a representative of LCSGJ Head Office. He is an Editor-in-Chief of the journal "LIVER CANCER". He is also serving as an Associate Editor or Editorial Board Member of numerous international journals.

He currently serves as a Secretary of Asian Federation of Societies for Ultrasound in Medicine and Biology (AFSUMB), and President of World Federation of Societies for Ultrasound in Medicine and Biology (WFUMB).

\section{Research Interest:}

Hepatology 


\section{Sorafenib in Patients with Liver Dysfunction: Final Analysis of GIDEON}

Masatoshi Kudo ${ }^{7}$, Riccardo Lencioni ${ }^{2}$, Sheng-Long Ye ${ }^{3}$, Jean-Pierre Bronowicki ${ }^{4}$, Xiao-Ping Chen ${ }^{5}$, Lucy Dagher ${ }^{6}$, Junji Furuse ${ }^{7}$, Jeff F. Geschwind ${ }^{8}$, Laura Ladrón de Guevara ${ }^{9}$, Christos Papandreou ${ }^{10}$, Arun J. Sanyal ${ }^{11}$, Tadatoshi Takayama ${ }^{12}$, Seung Kew Yoon ${ }^{13}$, Keiko Nakajima ${ }^{14}$, Alan Venook ${ }^{15}$, Jorge Marrero ${ }^{16}$

${ }^{1}$ Department of Gastroenterology and Hepatology, Kinki University School of Medicine, Osaka, Japan; ${ }^{2}$ Division of Diagnostic Imaging and Intervention, Pisa University Hospital and School of Medicine, Pisa, Italy; ${ }^{3}$ Liver Cancer Institute and Zhongshan Hospital, Fudan University, Shanghai, China; ${ }^{4}$ Department of Gastroenterology and Hepatology, INSERM U954, University Hospital of Nancy, University Henri Poincaré, Vandœuvre-lès-Nancy, France; ${ }^{5}$ Hepatic Surgery Center, Tongji Hospital, Tongji Medical College, Huazhong University of Science and Technology, Wuhan, China; ${ }^{6}$ Policlínica Metropolitana, Caracas, Venezuela; ${ }^{7}$ Kyorin University School of Medicine, Mitaka, Tokyo, Japan; ${ }^{8}$ Vascular and Interventional Radiology, Johns Hopkins University School of Medicine, Baltimore, MD, USA; ${ }^{9}$ Hospital Angeles Clínica Londres, Mexico City, Mexico; ${ }^{10}$ University Hospital of Larissa, Larissa, Greece; ${ }^{11}$ Virginia Commonwealth University Medical Center, Richmond, VA, USA; ${ }^{12}$ Department of Digestive Surgery, Nihon University School of Medicine, Tokyo, Japan; ${ }^{13}$ The Catholic University of Korea, Seoul, Korea; ${ }^{14} \mathrm{Global}$ Medical Affairs, Bayer HealthCare Pharmaceuticals, Montville, NJ, USA; ${ }^{15}$ University of California, San Francisco, CA, USA; ${ }^{16}$ Department of Internal Medicine, Division of Digestive and Liver Diseases, University of Texas Southwestern Medical Center, Dallas, TX, USA

Background: GIDEON is a prospective, non-interventional study. Completion of GIDEON creates a large, global database of $>3000$ Sor-treated unresectable HCC (uHCC) pts, allowing for evaluation of a broad pt population, including Child-Pugh (CP) B pts with more advanced liver dysfunction.

Methods: Baseline characteristics were collected in pts for whom a decision to treat with Sor had been made in clinical practice. Adverse events (AEs), dosing, and outcomes data were collected during follow-up.

\begin{tabular}{|c|c|c|c|c|c|c|}
\hline & \multicolumn{2}{|c|}{$\begin{array}{l}\text { Total } \\
\mathrm{n}=3202^{\mathrm{a}} \\
(100)\end{array}$} & \multicolumn{2}{|c|}{$\begin{array}{l}\text { CP-A } \\
n=1968 \\
(61.5)\end{array}$} & \multicolumn{2}{|c|}{$\begin{array}{l}\text { CP-B } \\
n=666 \\
(20.8)\end{array}$} \\
\hline & All & DR & All & DR & All & DR \\
\hline \multicolumn{7}{|l|}{ AEs, $\%^{b}$} \\
\hline All grades & 85.3 & 66.0 & 84.0 & 68.5 & 88.6 & 64.4 \\
\hline Grade $3 / 4$ & 31.7 & 23.5 & 32.4 & 25.6 & 31.5 & 21.9 \\
\hline SAEs & 43.3 & 9.3 & 36.0 & 8.8 & 60.4 & 14.1 \\
\hline \multicolumn{7}{|l|}{$A E$ rate (event per patient-year) ${ }^{b, c}$} \\
\hline Any AE & 1.61 & 1.24 & 1.44 & 1.17 & 2.14 & 1.55 \\
\hline Diarrhea & 0.58 & 0.51 & 0.54 & 0.48 & 0.71 & 0.62 \\
\hline Hand-foot skin reaction & 0.51 & 0.50 & 0.55 & 0.54 & 0.42 & 0.41 \\
\hline Fatigue & 0.45 & 0.29 & 0.38 & 0.27 & 0.62 & 0.34 \\
\hline Discontinuation due to AEs, $\%^{b}$ & 31.4 & & 28.9 & & 40.1 & \\
\hline Daily dose, median, $\mathrm{mg}^{\mathrm{d}}$ & 688.0 & & 677.0 & & 741.5 & \\
\hline $\begin{array}{l}\text { Treatment duration, median, } \\
\text { weeks }^{\mathrm{d}}\end{array}$ & 15.0 & & 17.6 & & 9.9 & \\
\hline
\end{tabular}

ancludes CP-C, 74 pts; not evaluable, 493 pts; ${ }^{\text {b}}$ Treatment-emergent; ${ }^{\mathrm{c}} \mathrm{Cal}-$ culated based on 365.25 days per year; ${ }^{\mathrm{d}} \mathrm{Pts}$ with dosing data.

Results: 3202 pts were evaluable for safety. Overall, the incidence of AEs and drug-related (DR) AEs was similar across CP subgroups, although serious AEs (SAEs) were more common in CP-B than CP-A pts. The rate of DR AEs (event per patient-year) was also comparable between CP-A and CP-B pts. The average daily Sor dose was slightly higher in $\mathrm{CP}-\mathrm{B}$ than CP-A pts; duration of treatment was longer in CP-A (Table). In the intent-to-treat population $(n=3213)$, median overall survival (OS) (months [95\% CI]) was longer in CP-A (13.6 [12.8-14.7]) than CP-B pts (5.2 [4.6-6.3]); time to progression was similar: CP-A (4.7 [4.3-5.2]); CP-B (4.4 [3.55.5]). Median OS was shorter in pts with a higher CP-B score: 7 (6.2 [4.9-8.7]); 8 (4.8 [4.1-6.9]); 9 (3.7 [3.0-5.1]).

Conclusions: Sor safety and dosing during treatment are generally consistent across pts irrespective of liver function. As anticipated, CP status is a strong prognostic factor for OS in uHCC pts. 


\section{Curriculum Vitae}

Name

Position

Institution

E-mail
Jong Young Choi

Division of Hepatology

Department of Internal Medicine

The Catholic University of Korea

jychoi@catholic.ac.kr

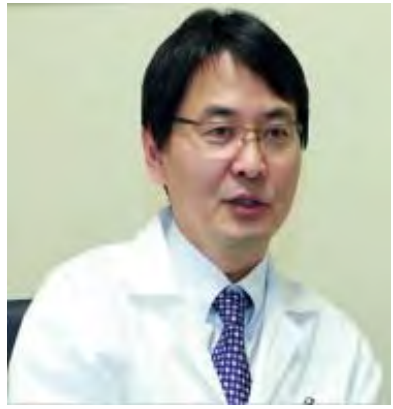

Dr. Jong Young Choi graduated from Catholic Medical College in 1987. And then, he completed a clinical fellowship in Kangnam St. Mary's Hospital. He received his PhD degree in Medical Science at Catholic University in 1997. He studied about DNA methylation as a visiting scientist in NIH between 1999 and 2000.

Professor Choi is currently a Professor at the Department of Internal Medicine, College of Medicine, The Catholic University of Korea since 2008.

Professor Choi has published 50 international scientific papers. He served as an Editor in Chief, The Korean Journal of Hepatology and General manager of Liver Cancer Study Group of Korea. Now, he serves as a member of Korean Association of Study of Liver Diseases and Korean Liver Transplantation Study Group. Currently, He is a secretary general of 2013 APPLE.

\section{Research Interest:}

His research interest is "liver transplantation in HCC and immune tolerance". Professor Choi also plays an important role as transplantation hepatologist. 


\section{Detection of Circulating Tumor Cell in Blood from Hepatocellular Carcinoma with Extrahepatic Metastasis}

\section{Jong Young Choi}

Department of Internal Medicine, Seoul St. Mary's Hospital, The Catholic University of Korea, Seoul, Korea

Recently, levels of circulating tumor cells (CTCs) in the blood of breast cancer patients have been measured to predict invasiveness and treatment response. Theoretically, the presence of CTCs in blood is in agreement with the hematogenous spread theory of metastasis. In addition, measurement of a blood biomarker is non-invasive and can be repeatedly used. The presence of CTCs in the peripheral blood of cancer patients has been recognized for more than a century. However, CTCs are rare, being as few as one cell per 109 hematologic cells in the blood of patients with metastatic cancer. The direct isolation of CTC in some forms of cancer may be difficult. Many methods of CTC isolation/detection have been attempted; these include immunomagnetic bead separation, filtration, density-gradient centrifugation, and PCR-based assays (RT-PCR: reverse-transcription PCR; qRT-PCR: quantitative real-time PCR). The metastasis related genes among genes expressed in HCC tissue were Snail, Twist, SIP1. Among them, Snail (Drosophila Snail 1 homolog) is closely associated with Wnt/b-catenin pathway, which is critical signaling pathway in hepatocarcinogenesis and that is known to be associated with epithelial mesenchymal tran- sition (EMT), major pathway in tumor metastasis. Snail represses transcription of E-cadherin and stimulates matrix metalloproteinase expression, thus allowing cells to detach from their neighbors and migrate, ultimately permitting metastasis to distant organs. Furthermore, the Snail was significantly expressed in several proteomic analyses of HCC tissue. Because previous studies suggested the possibility that Snail can be one of metastasis markers in patients with HCC, we conducted this clinical study with object of identifying circulating tumor cells and clinical meaning of Snail expression in blood from patients with HCC.

Sixty-six consecutive patients with HCC (30 without metastasis, 36 with metastasis) were prospectively enrolled, as were 30 with liver cirrhosis and 23 healthy subjects. CTCs were isolated by FACS using Ber-EP4 and anti-CD45 antibodies, and CTC identity confirmed by immunofluorescent cytokeratin staining. Snail mRNA levels were measured by quantitative real-time PCR of blood samples. CTCs, positive for pan-cytokeratin and Snail, were isolated from five HCC patients with metastasis. The mean amount of Snail mRNA in HCC with metastasis was 18.8-fold, 26.6-fold greater than HCC without metastasis, liver cirrhosis, respectively. When compared with healthy controls, the mean level of Snail mRNA in HCC without metastasis was 10.1-fold greater $(\mathrm{P} \backslash 0.001)$. In six patients showing complete remission of HCC, Snail mRNA decreased to levels similar to those of healthy controls. This study suggests the possibility that circulating Snail mRNA levels may have been associated with extra-hepatic metastasis in HCC patients. 
The 4th Asia-Pacific Primary Liver Cancer Expert Meeting

\section{Curriculum Vitae}

$\begin{array}{ll}\text { Name } & \text { Ann-Lii Cheng } \\ \text { Position } & \text { Professor \& Director } \\ \text { Institution } & \text { National Taiwan University Hospital } \\ \text { E-mail } & \text { alcheng@ntu.edu.tw }\end{array}$

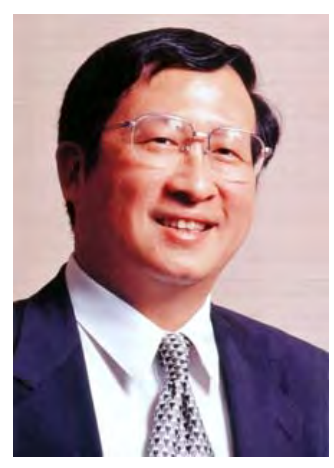

Ann-Lii Cheng is Professor and Chairman of the Department of Oncology of the National Taiwan University Hospital in Taipei, Taiwan. He received his MD degree, PhD degree, and his specialty training in Medical Oncology at the Medical School of the National Taiwan University. In 1990, he was a research fellow at the Comprehensive Cancer Center of the University of Wisconsin, Madison, USA. Dr. Cheng has been actively involved in basic and translational research in GI oncology, particularly hepatocellular carcinoma and gastric lymphoma, and has published more than 200 peer-reviewed articles. He received the "Outstanding Research Award" from the National Science Council of Taiwan, and the "Outstanding Cancer Research Award" from the Chinese Oncology Society. He became "Distinguished Professor" of National Taiwan University in 2006, and was elected as Fellow of American Association for the Advancement of Science (AAAS) in 2007. Dr. Cheng is an active member of the American Association for Cancer Research (AACR) and the American Society of Clinical Oncology (ASCO). He has served on the editorial boards of Oncology (Basel), Asia-Pacific Journal of Clinical Oncology, and Targeted Oncology - Biotherapies for the Clinicians in Oncology. In addition, Dr. Cheng is Chairman of the Chemotherapy Committee of the Taiwan Cooperative Oncology Group, of which he was one of the founding members. He received the national award of "outstanding contributions for science and technology" in 2008, and a national award for academic excellence in 2010. He served as president of the Taiwan Oncology Society during 20092011. He was elected as council member of International Liver Cancer Association (ICLA) in 2012.

\section{Research Interest:}

Translational research for hepatocellular carcinoma. 


\section{Treatment Strategy for Advanced Stage of HCC}

Ann-Lii Cheng

National Taiwan University Hospital, Taipei, Taiwan

The global consensus has been focused on the use of sorafenib for advanced-stage HCC. Beyond sorafenib, several alternatives are mentioned in Asian locoregional guidelines. For example, the APASL guideline allows systemic cytotoxic chemotherapy for selected patients with good general condition and liver function. The JSH guideline addresses the use of intra-arterial chemotherapy. Locoregional therapies, such as surgical resection, TACE, TARE, and radiotherapy are also recommended in selected suitable patients. While many of these alternative approaches have not been validated by robust clinical trials, it cannot be over-emphasized that the best treatment for individual patients needs to be personalized, and should be delivered only by highly experienced personnels. 


\section{Poster Oral}

\section{PO-01 \\ Clinical Characteristics and Long Term Survival of Hepatocellular Carcinoma: A Nationwide Cohort Study in Korea}

\author{
Dong Hyun Sinn 1,2, Geum-Youn Gwak', Yong-Han Paik', \\ Moon Seok Choi ', Joon Hyeok Lee ${ }^{7}$, Kwang Cheol Koh', \\ Seung Woon Paik', Byung Chul Yoo on behalf of the \\ Korean Liver Cancer Study Group \\ ${ }^{1}$ Department of Medicine, Samsung Medical Center, \\ Sungkyunkwan University School of Medicine, \\ Sungkyunkwan University School of Medicine, Seoul, \\ Korea; ${ }^{2}$ Department of Internal Medicine, Sanggye Paik \\ Hospital, Inje University School of Medicine, Seoul, \\ Korea
}

Background: The Korean Liver Cancer Study Group (KLCSG), which was founded in 1999 by a group of leading liver specialists, built a nationwide HCC cohort between 2003 and 2005 in order to collect unbiased information about clinical characteristics, treatment pattern and survival of HCC occurring in Koreans, and help to make an optimal management strategy of HCC.

Methods: Out of 31,521 new HCC cases that were registered at Korea Central Cancer Registry between 2003 and $2005,4,630$ cases $(14.7 \%$ of total HCC cases) were randomly abstracted from 32 hospitals nationwide, and followed until December 2011. After excluding 110 patients who met exclusion criteria, a total of 4,520 HCC patients were analyzed.

Results: Mean age at the diagnosis of HCC was $57.1 \pm 10.8$ years, and male comprised $81.0 \%$. HBV was a predominant etiology (72\%). Stage at diagnosis was 10\%, 43\%, 28\%, 11\% and $8 \%$ for mUICC stage I, II, III, IV-A and IV-B, respectively. The overall 1-, 3-, and 5-year survival rates were 56\%, 35\% and $27 \%$. Age (5-year survival rate: $30 \%$ vs. $22 \%$ for age $<60$ years vs. $\geq 60$ years), gender ( $32 \%$ vs. $25 \%$ for men vs. women), Child-Pugh Class $(35 \%, 11 \%$ and $11 \%$ for A, B and C, respectively), mUICC stage at diagnosis (52\%, 37\%, 15\%, 6\% and 6\% for stage I, II, III, IV-A and IV-B, respectively) and treatment modality $(61 \%, 25 \%$ and $7 \%$ for curative, palliative and no information, respectively) were independently related to survival. Also, etiology significantly affected clinical and tumor characteristics and survival. HBV-related HCC occurred at younger age and were detected at more advanced stage compared to HCV-related HCC. When adjusted, HBV-related HCC showed significantly poorer survival than HCV-related HCC [hazard ratio (95\% CI): 1.13 (1.01-1.28), p = 0.03].

Conclusion: This study provides a comprehensive picture of HCC with long-term survival in an HBV-endemic area, which could be used as a valuable reference for comparison for future study.

\section{PO-02 \\ Comparisons Between Hepatitis B and C Virus Related Hepatocellular Carcinoma: Analysis of 9285 Cases in a Single Center in Southern Taiwan}

\section{Sheng-Nan Lu, Jing-Houng Wang, Kwong-Ming Kee, Yuan-Hung Kuo}

Division of Hepatogastroenterology, Department of Internal Medicine, Kaohsiung Chang Gung Memorial Hospital and Chang Gung University College of Medicine, Kaohsiung, Taiwan

Background: Hepatitis B virus (HBV) and hepatitis C virus (HCV) are two major causes of hepatocellular carcinoma (HCC). We reported the difference between HBV- and HCVrelated HCC.

Methods: During 1992 and 2012, 9285 HCC cases visited our hospital. Based on the diagnosis year, patients were divided in to 7 periods, i.e. period (P) 1 (1992 1994), P2 (1995 1997), P3 (1998 2000), P4 (2001 2003), P5 (2004 2006), P6 (2007 2009) and P7 (2010 2012).

Results: Proportions of HBV-, dual B \& C, HCV- and NBNC HCC were $55.7 \%, 10.1 \%, 22.6 \%$ and $11.5 \%$, respectively, in P1. They changed with trends to $46.0 \%, 5.2 \%, 32.4 \%$ and $16.4 \%$ in P7. From P1 to P7, male to female ratios decreased from 4.8 to 3.0, and mean ages increased for 4 years in both HBV (53.5 to 57.7 years) and HCV (61.2 to 65.9 years) cases. Proportions of HBV cases presenting as BCLC stage 0 and A were $3.8 \%$ and $13.8 \%$ initially, and increased to $9.7 \%$ and $29.6 \%$ finally. Those of HCV cases were higher as $3.1 \%$ and $26.2 \%$ initially, and $15.7 \%$ and $39.5 \%$ finally. Single tumor presented in $50.9 \%$ and $53.1 \%$ of HBV and HCV cases, respectively. More HCV cases has tumor $<3 \mathrm{~cm}(50.1 \%$ vs. $34.7 \%)$. HCV cases had early BCLC staging and AJCC (7th edition) TNM staging. The rates of AST and ALT levels higher than 2XULN 
were $35.4 \%$ and $22.2 \%$ in HBV cases, and $41.9 \%$ and $32.8 \%$ in HCV cases. Both groups had similar rates of Child-Pugh class A $(66.9 \%$ and $67.1 \%)$. HCV cases had high comorbidity of Diabetes (33\% vs. $21.5 \%)$ and hypertension $(45.4 \%$ and $26.5 \%$ ). There was no difference between two groups in disease-free survival after tumor resection or radiofrequency ablation. HCV had slightly lower overall survival (HR: 1.2 (1.1 1.4), p < 0.001).

Conclusions: The report showed the discrepancy between HBV- and HCV-related HCC.

\section{PO-03 \\ Protein Induced by Vitamin K Antagonist-II (PIVKA-II) is Reliable Prognostic Factor in Small Hepatocellular Carcinoma}

Jong Man Kim, Jin Yong Choi, Wontae Cho, Shin-Seok Yang, Seunghwan Lee, Hyunghwan Moon, Milljae Shin, Jae Berm Park, Choon Hyuck David Kwon, Sung Joo Kim, Jae-Won Joh, Suk-Koo Lee

Department of Surgery, Samsung Medical Center, Sungkyunkwan University School of Medicine, Seoul, South Korea

Background: Hepatocellular carcinoma $(\mathrm{HCC})<2 \mathrm{~cm}$ in diameter has a favorable prognosis, so surgical resection of small HCC is associated with good outcomes. However, the predisposing factors of prognosis following resection of HCC remain ill-defined. The aims of this study were to identify the clinicopathologic characteristics and outcomes of patients with small HCC and analyze the predisposing factors for tumor recurrence after surgery.

Methods: We retrospectively reviewed 180 patients with small HCC who underwent hepatectomy between 2006 and 2010. Independent predictors of tumor recurrence were identified using Cox regression analysis.

Results: The 1-year, 3-year, and 5-year disease-free survival rates and overall survival rates were $83.7 \%, 68.0 \%$, $65.3 \%$ and $98.9 \%, 96.5 \%, 92.7 \%$, respectively. Multivariate analysis reported that Protein Induced by Vitamin K Antagonist-II (PIVKA-II) $\geq 200 \mathrm{mAU} / \mathrm{mL}$, alkaline phosphatase $(\mathrm{ALP}) \geq 80 \mathrm{IU} / \mathrm{mL}$, and microvascular invasion were important predisposing factors for tumor recurrence. Elevated serum PIVKA-II level was associated with microvascular invasion in small HCC, which was a powerful predisposing factor.

Conclusions: Although small HCC is generally associated with a good prognosis, serum PIVKA-II level $\geq 200 \mathrm{mAU} / \mathrm{mL}$, $\mathrm{ALP} \geq 80 \mathrm{IU} / \mathrm{L}$, and microvascular invasion were predisposing factors for tumor recurrence. These factors can be used to stratify patients with respect to recurrence after resection. Elevated PIVKA-II was closely associated with microvascular invasion in small HCC. These data emphasize the importance of PIVKA-II in small HCC.

\section{PO-04 \\ Functional Significance, Clinical Relevance and Therapeutic Implications of Annexin A3 (ANXA3) in CD133+ Cancer Stem Cells-Driven Hepatocellular Carcinoma}

\section{Man Tong, Xin-Yuan Guan, Stephanie Ma}

Department of Clinical Oncology, State Key Laboratory for Liver Research, Faculty of Medicine, The University of Hong Kong, Hong Kong

Background: We have previously reported that the tumor growth of hepatocellular carcinoma (HCC) is fuelled, at least in part, by a small subset of cancer stem cells (CSCs) marked by the CD133 phenotype. Our present study aims at delineating the molecular mechanism by which these cells mediate tumor formation, progression and interaction with its tumor niche, as well as developing a novel diagnostic biomarker and therapy for the detection and treatment of the disease.

Methods: RNA-Seq was employed for the systematic identification of differentially expressed genes between $\mathrm{CD} 133^{+}$liver CSCs and CD133- differentiated counterparts. Functional studies in vitro and in vivo were performed to study the role of the selected candidate gene in conferring cancer and stem cell-like properties. A novel neutralizing antibody against the candidate gene was developed and tested for its application as a therapeutic treatment against HCC.

Results: By RNA-Seq, annexin A3 (ANXA3) was found to be most significantly up-regulated in the $\mathrm{CD} 133^{+}$subset. Both endogenous and secretory levels of ANXA3 were found to be positively correlated with CD133 and significantly up-regulated in HCC clinical samples. ANXA3 overexpression was also tightly associated with advanced tumor stage. Functional studies involving lentiviral-based knockdown and overexpression approaches found ANXA3 to regulate various cancer and stem cell-like properties including tumor initiation, migration, invasion, angiogenesis, self-renewal and resistance to chemotherapy and apoptosis. Subsequent gene expression profiling by a cDNA microarray in two ANXA3 repressed cell lines found ANXA3 to be functionally involved in driving $\mathrm{CD}_{133^{+}}$liver CSCs via a deregulated JNK/AP-1 pathway. Neutralization of ANXA3 in $\mathrm{CD}_{133^{+}}$HCC using a newly developed monoclonal antibody dramatically inhibited cell proliferation, migration, angiogenesis and sphere formation.

Conclusions: ANXA3 confers both cancer and stem celllike properties in $\mathrm{CD} 133^{+}$liver CSCs through a deregulated JNK pathway. With further study, ANXA3 may possibly hold diagnostic and therapeutic values in HCC. 


\section{PO-05 \\ Clinicopathologic Feature and Prognosis of Combined Hepatocellular- Cholangiocarcinoma with Stem Cell Feature}

Hwakyung Jung, Dong-Shik Lee, Sung-Su Yun

Yeungnam University Medical Center, Korea

Introduction: Combined hepatocellular-cholangiocarcinoma (cHCC-CC) is rare subtype of primary liver cancer, and generally the prognosis is known to be worse than HCC. The purposes of this study were to evaluate the clinicopathologic feature and prognosis of the cHCC-CC.

Materials and Methods: From January 2000 to December 2012, 33 patients were diagnosed with cHCC-CC who underwent hepatectomy for liver malignancy at the Yeungnam university hospital. The clinicopathologic and follow-up data were evaluated retrospectively, and compared the data of patients with HCC who underwent the operation.

Results: $33(8.51 \%)$ patients of the 388 patients who were initially diagnosed with HCC was diagnosed with cHCC-CC after surgery from January 2000 to December 2012. Mean age was $57.85 \pm 11.39$ (26 78). Mean R15=15.74 \pm $10.98 \%$. Preoperatively only 3 patients (9.1\%) had been suspected with cHCC-CC in CT. 4 patients $(12.1 \%)$ showed T1-low, T2-high intensity mass lesion on MRI, so unusual pattern of HCC or CCC was suspected. 12 patients (36.4\%) showed unusual pattern of arterial enhancement on CT and angiography. cHCC-CC was categorized with 2010 WHO classification, 2 patients (6.1\%) were classical subtype, 1 patient $(3.0 \%)$ was typical subtype, 6 patients $(18.2 \%)$ were intermediate type, 3 patients $(9.1 \%)$ were cholangiolocellular type. 21 patients were unclassified. The mean value of preoperative serum-AFP was $4398.86 \pm 14763.43$ (1 77249). Lymph node metastasis was present in 4 patient $(12.1 \%)$ and 2 patient (1\%) for cHCC-CC and HCC, respectively. The cumulative 1, 3 and 5 year disease-free survival rates after hepatectomy were $64.4 \%, 37.9 \%$, and $0 \%$ in cHCC-CC patients, and $80.9 \%$, $62.6 \%, 52.0 \%$ in HCC patients. The cumulative 1,3 and 5 year survival rates were $72.1 \%, 46.5 \%$ and $31.0 \%$ in cHCC-CC patients, and $78.0 \%, 56.4 \%, 46.0 \%$ in HCC patients. But, after adjusted by stage, there was no significant difference in overall survival and disease-free survival.

Conclusion: $\mathrm{CHCC}-\mathrm{CC}$ have a clear pattern of HCC, but it has also some aspect of CCC, such as lymph node metastasis. Survival difference may be related to higher rate of lymph node metastasis and advanced stage of cHCC-CC.

\section{PO-06 \\ Actionable Gene Expression-Based Patient Stratification for Molecular Targeted Therapy in Hepatocellular Carcinoma}

Jung-Hee Kwon ${ }^{1}$, Namgyu Lee ${ }^{2}$, Jin Young Park ${ }^{1}$, Yun Suk Yu', Jin Pyo Kim ${ }^{7}$, Ji Hye Shin ${ }^{2}$, Dong-Sik Kim³, Jae Won Joh', Dae Shick Kim ${ }^{5}$, Kwan Yong Choi², Koo-Jeong Kang ${ }^{6}$, Gundo Kim ${ }^{7}$, Young Ho Moon ${ }^{1 *}$, Hee Jung Wang ${ }^{8 *}$

${ }^{1}$ Cbs Bioscience Inc., Daejeon, Korea; ${ }^{2}$ Department of Life Sciences, Pohang University of Science and Technology, Pohang, Korea; ${ }^{3}$ Division of HBP Surgery \& Liver Transplantation, Department of Surgery, Korea University College of Medicine, Seoul, Korea; ${ }^{4}$ Department of Surgery, Samsung Medical Center, Sungkyunkwan University School of Medicine, Seoul, Korea; ${ }^{5}$ Department of Pathology, Samsung Medical Center, Sungkyunkwan University School of Medicine, Seoul, Korea; ${ }^{6}$ Department of Surgery, Keimyung University School of Medicine, Daegu, Korea; ${ }^{7}$ Department of Microbiology, Pukyong National University, Busan, Korea; ${ }^{8}$ Department of Surgery, Ajou University School of Medicine, Suwon, Korea

Background: The effectiveness of molecular targeted agents is modest in hepatocellular carcinoma (HCC). Efficacy of molecular targeted therapies has been better in cancer patients with high expression of actionable molecules defined as cognate target molecules. However, patient stratification based on the actionable molecules dictating the effectiveness of targeted drugs has remained understudied in HCC.

Methods: Paired tumor and non-tumoral tissues derived from a total of 130 HCC patients were studied. Real-time RT-PCR was used to analyze the mRNA expression of actionable molecules in the tissues. Frequency and timing of gene expression were examined. Hierarchical clustering analyses were performed for patient stratification. In vitro sensitivity to sorafenib was tested in HCC cell lines.

Results: mRNA levels of EGFR, VEGFR2, PDGFR $\beta$, FGFR1, and mTOR were up-regulated in tumors compared to non-tumors in $35.4,42.3,61.5,24.6$, and $50.0 \%$ of patients, respectively. Up-regulation of EGFR was observed at early stage and tended to gradually decrease toward late stages (BCLC stage A: 41.9\%; B: 30.8\%; C: $17.6 \%$ ). Frequency of VEGFR2 expression in tumors at stage $C$ was lower than that in the other stages (BCLC stage A: 45.9\%; B: 41.0\%; C: $29.4 \%)$. PDGFR $\beta$ and mTOR were observed to be up-regulated in more than $50 \%$ of tumors in all the stages whereas FGFR1 was up-regulated in only about $20 \%$ of HCC irrespective of stages. A cluster analysis of actionable gene expression revealed that HCC can be categorized into different subtypes that predict the effectiveness of molecular targeted agents and combination therapies in clinical trials. HCC cells with up-regulation of PDGFR $\beta$ and c-Raf mRNA are more susceptible to sorafenib treatment in a dose and time- 
dependent manner than cells with low expression of the genes.

Conclusions: mRNA expression analysis of actionable molecules could provide the rationale for new companion diagnostics-based therapeutic strategies in the treatment of HCC.

\section{PO-07 \\ Expressions of Eukaryotic Translation Initiation Factor $3 \mathrm{~m}$ and Macrophage Migration Inhibitory Factor in Hepatocellular Carcinoma

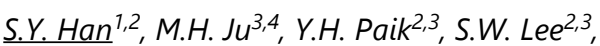 J.S. Jeong $2,3,4$}

Department of ${ }^{1}$ Internal Medicine, ${ }^{2}$ Liver center, ${ }^{3}$ Pathology, and ${ }^{4}$ Immune-network Pioneer Research Center, Dong-A University College of Medicine, Busan, Republic of Korea

Background: Eukaryotic translation initiation factor 3(eIF3) has been reported to be an important factor to regulation of translation initiation rate and oncogenic roles. Function of lastly defined eIF3 subunit (eIF3m) has not yet characterized. Recently, it is reported that eIF3m expression affects cell proliferation and cell cycle progression in colon cancer and one of tumorigenesis-related genes, macrophage migration inhibitory factor (MIF) is affected by eIF3m. In this study, we investigated expressions of eIF3m and MIF in human HCCs via immunohistochemistry and their relations with clinicopathological parameters, pursuing their roles in development and progression during hepatocarcinogenesis.

Methods: Surgically resected HCCs from 114 patients were selected. Immunohistochemistry was processed with anti-eIF3m and anti-MIF antibodies using auto-immunostainer.

Results: One hundred HCCs $(87.7 \%)$ showed eIF3m expression, composing low grade $20.2 \%$, intermediate $45.6 \%$ and high grade $21.9 \%$. When focally expressed, eIF3m was predominant at peripheries and invasive fronts of tumor nests. Higher expression was significantly associated with high preoperative AFP level, poor differentiation and septal forrmation $(P=<0.0001,0.0001,0.0055)$. Fatty change, monouclear cell infiltration, vascular invasion and progression tended to be associated with higher expression $(P=0.0544$, $0.0588,0.0584,0.0817)$. MIF expression was detected in 103 of 114 HCCs (90.4\%), consisting of low grade $43.0 \%$ and high grade $47.4 \%$. High expression was significantly associated with high preoperative AFP level and encapsulation $(P=$ $0.0123,0.0142)$. Vascular invasion tended to be associated with high expression $(P=0.0910)$. A significant positive correlation was found between eIF3m and MIF expressions $(P=$ 0.0048). High expression of MIF tended to be related with high recur probability $(P=0.0526)$. Multivariate analysis identified that eIF3m or MIF expression was not an independent predictive factor for recur.

Conclusions: It is concluded that eIF3m expression suggested with downstream regulation of MIF expression may play a pivotal role in progression and invasion in human HCC. MIF expression may play an important role in dismal prognosis of HCC patients.

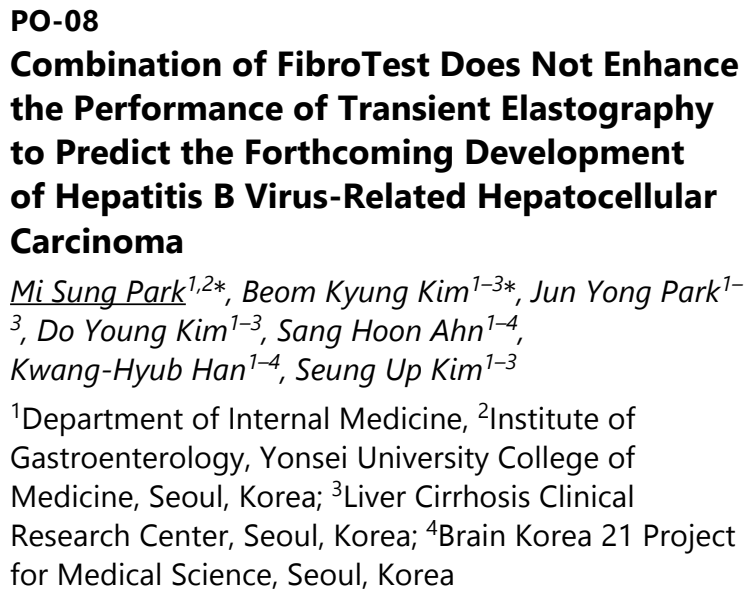

Background: Liver stiffness (LS) measurement using transient elastography (TE) and FibroTest (FT) have been validated as alternatives to liver biopsy (LB) in assessing liver fibrosis in patients with chronic hepatitis B (CHB). We aimed to assess the prognostic value of the combined use of LS and FT in predicting liver-related events (LREs) in patients with CHB.

Methods: We retrospectively analyzed consecutive patients with CHB who underwent LB and measurement of LS and FT on the same day from January 2007 to December 2010. Liver fibrosis was evaluated by the Batts and Ludwig scoring system. LRE was defined as hepatic decompensation, hepatocellular carcinoma (HCC), and liver-related deaths.

Results: A total of 154 patients (88 male and 66 female) were analyzed. The median age was 48 years. During followup period (median 50.9 months), 18 (11.7\%) patients developed LRE (decompensation in 3 patient and HCC in 15 patients). Median time to LRE was 25.1 (range, 5.9-85.2) months. The area under the receiver operating characteristic curve (AUROC) of LS, FT, LS+FT, LSxFT, and histological fibrosis staging in predicting LRE at 5-year follow-up was 0.740 (95\% confidence interval [CI], 0.625-0.856), 0.628 (95\% CI, 0.516-0.740), 0.739 (95\% CI, 0.624-0.855), 0.731 (95\% CI, 0.608-0.853) and 0.661 (95\% CI, 0.538-0.785), respectively. After adjustment of age and clinically diagnosed liver cirrhosis which were significant in univariate analysis, LS, LS+FT, and LSxFT independently predicted LRE $(P=0.009$, HR 1.050, 95\% CI 1.012-1.090; $P=0.009$, HR 1.051, 95\% CI 1.012-1.091; $P=0.011$, HR 1.084, 95\% CI 1.019-1.153, 
respectively), whereas FT was not significant even in univariate analysis $(P=0.094)$.

Conclusions: LS had a better prognostic value in predicting LRE in patients with CHB than FT or histological fibrosis staging. In addition, combination of FT with LS did not improve the prognostic performance of LS.

\section{PO-09 \\ The Comparison of Overall Survival Between Child C Early Stage HCC and Child A Advanced Stage HCC}

Jin Nyoung Kim ${ }^{1}$, Jae Young Jang*1, Soung Won Jeong ${ }^{1}$, Woong Cheul Lee ${ }^{1}$, Eui Ju Park', Byoung Moo Lee ${ }^{1}$, Sung Woo Ryu' ${ }^{7}$, Ji Hye Kim ${ }^{7}$, Sae Hwan Lee ${ }^{7}$, Sang Gyune Kim ${ }^{1}$, Sang-Woo Cha ${ }^{1}$, Young Seok Kim ${ }^{1}$, Young Deok Cho ${ }^{1}$, Hong Soo Kim ${ }^{1}$, Boo Sung Kim ${ }^{1}$

${ }^{1}$ Institution for Digestive Research, Digestive

Disease Center, Department of Internal Medicine, Soonchunhyang University Hospital, Seoul,

Republic of Korea

Background/Aim: The prognosis of hepatocellular carcinoma (HCC) is affected by stage as well as liver function. We analyzed the outcome of early stage HCC in Child-Pugh class $\mathrm{C}$ patients and advanced HCC in Child-Pugh class A patients.

Methods: Among 453 HCC patients, group I included consecutive 33 patients with early stage (I, II) in Child-Pugh class $\mathrm{C}$ and Group II included 45 consecutive patients with advanced stage (III, IV) in Child-Pugh class A. We compared clinical characteristics, complications of cirrhosis, overall survival between two groups.

Results: Younger age, male, presence of $\mathrm{HBV}$, $\alpha$-fetoprotein (AFP) ( $>400 \mathrm{ng} / \mathrm{ml})$, and MELD score $(<15)$ were significantly high in advanced stage HCC in Child-Pugh class A patients (Group II) $(p<0.05)$. Complications of cirrhosis such as variceal hemorrhage, ascites, and hepatic encephalopathy were increased in the Group I $(p<0.05)$. Median overall survival was 24.5 months (95\% CI 10.6-38.4) for Group I and 13.5 months (95\% CI 4.6-22.3) for Group II ( $p=$ 0.281 ). AFP was an independent prognostic factor in early stage in Child-Pugh class C patients (Group I). MELD score and tumor stage were independent prognostic factors in advanced stage in Child-Pugh class A patients (Group II). Conclusion: Overall survival was not different between two groups. AFP was a prognostic factor in early stage in Child-Pugh class $C$ patients (Group I), whereas MELD score and tumor stage were prognostic factors in advanced stage in Child-Pugh class A patients (Group II).

\section{PO-10 \\ Evaluation of Preoperative Prognostic Factors Regarding Prediction in Tumor Recurrence for Hepatocellular Carcinoma}

Jae Hyun Han, Gun Hyung $\mathrm{Na}$

Seoul St. Mary's Hospital, The Catholic University of

Korea, Seoul, South Korea

Background: Surgical resection has been known as the gold standard of treatment for hepatocellular carcinoma (HCC). However, tumor recurrence after surgical resection has been obstacle for long term survival so, the appropriate patient selection would be very important. Authors evaluated preoperative prognostic factors for tumor recurrence to refine the patient selection.

Methods: From January 2000 to June 2012, we analyzed retrospectively 304 patients who had undergone surgical resections for HCC with curative intention at our hospital. All patients were confirmed by HCC with safe resection margin by pathology. Six patients were excluded because of post-operative mortality. We analyzed the influencing factors to recurrence and the prediction factors for tumor biology.

Results: 298 patients were enrolled in this study. Among them, we experienced $129(43.3 \%)$ recurrences. 114 patients (88.4\%) were recurred in 2 years after surgery. The 5year and 10 year disease free survival and overall survival rate were $47.0 \%, 39.6 \%$ and $46.5 \%, 37.6 \%$ respectively. In multivariate analysis, alpha-fetoprotein (AFP) $>100 \mathrm{ng} / \mathrm{ml}$ ( $\mathrm{p}=0.035$ ), standardized uptake values (SUVs) of positron emission tomography - computed tomography (PET-CT) $>3.5(\mathrm{p}=$ $0.019)$ were prediction factor to tumor biology. Male patients $(\mathrm{p}=0.008), \mathrm{AFP}>100 \mathrm{ng} / \mathrm{ml}(\mathrm{p}=0.001)$, SUVs $>3.5$ and tumor size $>5 \mathrm{~cm}(\mathrm{p}=0.008)$ were analyzed as predisposing factors for recurrence. The tumor size $>5 \mathrm{~cm}$, AFP $>100 \mathrm{ng} / \mathrm{ml}$ and SUVs $>3.5$ was also significantly associated with survival. We also set scoring system to predict prognosis using AFP, SUVs and relative enhancement ratio (RER) calculated from magnetic resonance imaging (MRI). It predicted survival $(\mathrm{p}=$ $0.005)$ well but not recurrence $(p=0.227)$.

Conclusions: Careful preoperative evaluation and surgical resection should be needed especially in patients with AFP $>100 \mathrm{ng} / \mathrm{ml}$, SUVs $>3.5$ and RER $<0.6$. In order to stratify patient with respect to tumor recurrence after surgical resection, a prospective study using these parameters will be required. 


\section{PO-11 \\ Usefulness of Add-On Primovist-MRI on BCLC Staging Workup in Hepatocellular Carcinoma Patients Who were Candidates for Curative Treatments on Liver Dynamic CT: A Preliminary Prospective Single Center Study Young-Joo Jin ${ }^{1}$, Jin-Woo Lee ${ }^{1 *}$, Hyun Jung Chung ${ }^{1}$, Young soo Kim', Soon Gu Cho', Yong Sun Jeon ${ }^{2}$ \\ ${ }^{1}$ Department of Internal Medicine, Inha University Hospital, Inha University School of Medicine, Incheon, Republic of Korea; ${ }^{2}$ Department of Radiology, Inha University Hospital, Inha University School of Medicine, Incheon, Republic of Korea}

Background: The authors had previously reported about the utility of Primovist-enhanced magnetic resonance image (MRI) on the initial staging workup of patients with Barcelona Clinic Liver Cancer (BCLC) 0 or A staged hepatocellular carcinoma (HCC) using the retrospective study. In this study, we intended to validate the role of Primovist-enhanced MRI on BCLC staging workup of HCC using a preliminary prospective single center study.

Methods: A total of 33 patients who were initially diagnosed as BCLC stages 0 or A HCC without other intrahepatic suspicious lesions based on liver dynamic CT at our institution between August 2012 and March 2013 were prospectively enrolled. We evaluated whether an additional examination of Primovist-enhanced MRI could affect on BCLC stage, directly comparing to liver dynamic CT.

Results: Of the 33 patients, 14 (42.4\%) and 19 (57.6\%) had BCLC stages 0 and A HCC before Primovist-enhanced MRI, respectively. After Primovist-enhanced MRI, new HCC was detected in 7 patients. BCLC stage was up-staged in 6 patients [0àA $(n=3)$, AàB $(n=3)]$, but was not changed in 1 patients within stage A. In the remaining 26 patients without new HCC, tumor size was slightly increased on Primovist-enhanced MRI in 2 patients (stage 0àA). After add-on Primovist-enhanced MRI, 8 (24.2\%) of the 33 patients showed shift of BCLC stage with up-staging from stage 0 to $A(5 / 14,35.7 \%)$ or from stage A to $B(3 / 19,15.8 \%)$.

Conclusions: Primovist-enhanced MRI can provide additional information regarding the BCLC staging during initial staging workup in patients who were candidates for curative treatments by liver dynamic CT, despite absence of other suspicious HCC lesions.
PO-12

Alpha-Fetoprotein in Patients with Chronic Hepatitis B Under Antiviral Therapy

Jae-Jun Shim, Sun-Hee Park, Dong-Hyun Kim, Hyuk Kim, Tae-Young Kim, Seok Ho Dong, Byung-Ho Kim

Department of Internal Medicine, Kyung Hee University School of Medicine, Seoul, Korea

Background: Alpha-fetoprotein (AFP) is limited due to high false positive rate especially in active chronic hepatitis B. Recently, oral antiviral agents have shown high virological and biochemical responses in those patients. We investigated AFP level before and after antiviral therapy and its usefulness in patients with chronic hepatitis B.

Methods: Among 859 patients with chronic hepatitis B under antiviral therapy, patients more than 40 years with baseline ALT $>40 \mathrm{U} / \mathrm{L}$ and HBV-DNA > 2,000 IU/mL were included. Exclusion criteria were concurrent hepatocellular carcinoma (HCC), new HCC within 6 months, chronic hepatitis $\mathrm{C}$, and Child-Pugh class C. Baseline clinical and laboratory parameters were followed up in 6 months and 12 months after initiation of oral antiviral agent(s).

Results: Of 120 patients enrolled, males were 70 (58\%) and mean age was $50.9 \pm 8.3$. Seventy one patients had liver cirrhosis (59.2\%). Ninety six (79.3\%) were naïve patients and were treated with entecavir $(n=79)$ or lamivudine $(n=17)$. Remaining patients with a history of prior antiviral therapy were retreated with adefovir $(n=11)$, entecavir $(n=2)$ or combination of adefovir plus a nucleoside $(\mathrm{n}=11)$. Baseline ALT was $209 \pm 258 \mathrm{IU} / \mathrm{L}$. Following antiviral therapy, ALT normalization rate was $69.0 \%$ in 6 months and $79.6 \%$ in 12 months. Undetectable HBV-DNA rate was $41.0 \%$ in 6 months, $66.2 \%$ in 12 months. Proportion of patients with elevated AFP ( $\geq 20 \mathrm{ng} / \mathrm{mL}$ ) was $22.5 \%, 10.1 \%$, and $4.2 \%$ in baseline, 6 months, and 12 months, respectively. Among patients with normalized ALT ( $\mathrm{n}=75)$ in 12 months, only 1 patient had elevated AFP (1.3\%). HCC was detected in this patient 6 months later. During follow up of mean 36 months, 8 patients were diagnosed as new HCCs (6.7\%). Five patients had elevated AFP at the time of diagnosis. AFP was not normalized during 1 year in 3 out 5 patients. The other two patients showed abrupt elevation of AFP at 18 and 96 months, respectively.

Conclusions: In the era of oral antiviral agent, AFP might be a useful biomarker for HCC surveillance in patients with chronic hepatitis B, especially, who achieved biochemical response. 


\section{PO-13 \\ Correlation Between Enhancement Pattern of Hepatocellular Carcinoma on Real-Time Contrast-Enhanced Ultrasonography and Tumor Cellular Differentiation on Histopathology: Prospective Preliminary Study}

Woong Cheul Lee ${ }^{1}$, Jae Young Jang ${ }^{7 *}$, Jin Nyoung Kim ${ }^{7}$, Soung Won Jeong', Eui Ju Park', Byoung Moo Lee', Sung Woo Ryu', Ji Hye Kim ', Yun Nah Lee ${ }^{3}$, Sae Hwan Lee', Sang Gyune Kim ${ }^{3}$, Sang-Woo Cha', Young Seok Kim ${ }^{3}$, Young Deok $\mathrm{Cho}^{7}$, Hong Soo Kim², Boo Sung Kim ${ }^{7}$

${ }^{1}$ Institute for Digestive Research, Digestive Disease Center, Department of Internal Medicine, College of Medicine, Soonchunhyang University, Seoul, South Korea; ${ }^{2}$ Department of Internal Medicine, College of Medicine, Soonchunhyang University, Cheonan, South Korea; ${ }^{3}$ Department of Internal Medicine, College of Medicine, Soonchunhyang University, Bucheon, South Korea

Background: The objective of this study was to evaluate the correlation between the enhancement pattern of hepatocellular carcinoma (HCC) on contrast-enhanced ultrasonography (CEUS) and tumor cellular differentiation on histopathology.

Methods: Twelve patients with HCC underwent hepatic resection and liver biopsy were prospectively evaluated with CEUS and histopathological examination. CEUS was performed with SonoVue ${ }^{\circledR}$ and contrast pulse sequencing. Histopathological diagnosis were made according to the Edmonson grading system.

Results: Significant differences were shown between the time that the HCC became isoenhancing in PV (portal-venous) phase and tumor cellular differentiation (well differentiation $v s$. moderate to poor differentiation: $33 \mathrm{sec}(31,37.5)$ vs. 70 $\sec (64,80)$, median (IQR)) $(p=0.03)$, but not with the time of commencement of hyperenhancing or commencement of hypoenhancing in arterial phase and late phase $(p=0.343, p=$ 0.149 ).

Conclusions: The timing of HCC becoming isoenhancing on CEUS is correlated with tumor cellular differentiation; well differentiated tumors wash out more early than moderate or poorly differentiated ones.

\section{PO-14 \\ The Predictive Factors for the Response of Transarterial Chemoembolization on Contrast-Enhanced Ultrasonography}

Eui Ju Park', Jae Young Jang ${ }^{1 *}$, Soung Won Jeong ${ }^{1}$, Jin Nyoung Kim ', Woong Cheul Lee ${ }^{7}$, Byoung Moo Lee?, Sung Woo Ryu', Ji Hye Kim?', Sae Hwan Lee ${ }^{7}$, Sang Gyune Kim ${ }^{1}$, Sang-Woo Cha ${ }^{1}$, Young Seok Kim ${ }^{1}$, Young Deok Cho ${ }^{1}$, Hong Soo Kim ${ }^{7}$, Boo Sung Kim ${ }^{7}$

${ }^{1}$ Institution for Digestive Research, Digestive

Disease Center, Department of Internal Medicine, Soonchunhyang University Hospital, Seoul, Republic of Korea

Background/Aim: Hepatocellular carcinoma (HCC) response after transarterial chemoembolization (TACE) depends on tumor vascularization and characterization, which may be reflected in the pathognomonic pattern of HCC on contrast-enhanced ultrasonography (CEUS). We assessed predictive factors on CEUS for the response of TACE.

Methods: Seventeen patients with 18 HCC underwent TACE. All of the tumors were studied with CEUS before TACE. A second-generation echo-enhancer (Sono Vue; Bracco, Milan, Italy) and a low mechanical index technique were used. After TACE, tumor response was classified with a score between 1 and 4 according to the percentage of remaining enhancing tumor portion (score 1; remained enhancing lesion $<25 \%$, score $2 ; 25 \% \leq$ enhancing lesion $<50 \%$, score $3 ; 50 \% \leq$ enhancing lesion $<75 \%$, score 4 ; enhancing lesion $\geq 75 \%$ ). Score 1 was defined as "good response" after TACE. Tumor size, initial arterial enhancing time, arterial enhancing duration, intensity of arterial enhancement, presence of hypoenhanced pattern, and feeding artery to the tumor were analyzed.

Results: The median size of tumor was $3.1 \mathrm{~cm}$ (range 1-14 cm). The median time of initial arterial enhancing time and arterial enhancing duration were $21 \mathrm{sec}$, and $27.5 \mathrm{sec}$, respectively. Six tumors showed strong arterial enhancement. The presence of hypoenhanced pattern were shown in 14 tumors. Fifteen tumors had feeding arteries. The number of tumor response score after TACE in all tumors were 11 tumors in score 1, 4 in score 2, 2 in score 3, and 1 in score 4 . In predicting good response after TACE, initial arterial enhancing time, arterial enhancing duration, intensity of arterial enhancement, and hypoenhanced pattern did not show any significance. Presence of a feeding artery and tumor size $(<5 \mathrm{~cm})$ were predictive factors for good response $(p=0.043$, $p=0.047$ ).

Conclusions: The presence of a feeding artery and tumor size less than $5 \mathrm{~cm}$ were the predictive factors for the good response of TACE on CEUS. 


\section{PO-15 \\ Alpha-Fetoprotein as a Screening Marker for the Detection of Hepatocellular Carcinoma in Patients with Chronic Hepatitis B Virus Infection and Persistent Normal ALT Levels \\ Sun Young Yim Yeon Seok Seo*, Soon Ho Um, Yoon Tae Jeen, Hong Sik Lee, Hoon Jai Chun, Chang Duck Kim, Ho Sang Ryu \\ Department of Internal Medicine, Korea University College of Medicine, Seoul, Korea}

Background: Serum alpha-fetoprotein (AFP) is widely used as a screening test for the detection of hepatocellular carcinoma (HCC) in patients with high risk group. However, serum AFP level can be elevated without HCC. We aimed to study the role of AFP as screening marker for detecting HCC in different ALT levels.

Methods: Our study is based on 701 patients with chronic HBV infection. Diagnostic efficacy of AFP according to the presence of elevated ALT levels was analyzed.

Results: The age was $51.1 \pm 12$.8 years with male predominance $65.6 \%$. HCC were initially diagnosed in 103 patients (14.7\%) and serum AFP level was $7000.3 \pm 84644.2 \mathrm{ng} / \mathrm{mL}$ in these patients. The AUC of AFP for the detection of HCC was 0.847 and sensitivity/specificity of AFP cutoff values of 20, 200 and $400 \mathrm{ng} / \mathrm{mL}$ were $68.0 \% / 83.2 \%, 47.6 \% / 95.5 \%$, and $41.7 \% / 97.2 \%$, respectively. The initial AFP levels for patients without HCC was $53.3 \pm 282.1 \mathrm{ng} / \mathrm{mL}$ which showed significant correlation with serum ALT levels (Spearman's correlation coefficient, $0.463 ; P<0.001$ ). Therefore, changes in AFP level according to the changes in ALT levels were studied in patients who experienced serum ALT elevation during followup. AFP levels at ALT elevation, after normalization, and 3, 6, 9, 12 months thereafter were $104.6 \pm 381.7 \mathrm{ng} / \mathrm{mL}, 13.5 \pm$ $23.8 \mathrm{ng} / \mathrm{mL}, 6.0 \pm 7.6 \mathrm{ng} / \mathrm{mL}, 4.4 \pm 4.2 \mathrm{ng} / \mathrm{mL}, 4.1 \pm 2.9 \mathrm{ng} / \mathrm{mL}$ and $3.3 \pm 2.2 \mathrm{ng} / \mathrm{mL}$, respectively. AFP level was $2.9 \pm 1.6 \mathrm{ng} /$ $\mathrm{mL}$ in patients whose ALT level sustained within normal limit for at least 1 year. Patients without HCC were followed up for $63.3 \pm 38.0$ months and HCC were detected in 31 patients. AFP increased significantly from $4.0 \pm 1.8 \mathrm{ng} / \mathrm{mL}$ to $30.8 \pm 44.5 \mathrm{ng} /$ $\mathrm{mL}$ when HCC was diagnosed in these patients $(P<0.001)$. AUC was 0.951 in diagnosing HCC and the sensitivity/specificity of AFP cutoff values for 5.0, 10.0 and $15.0 \mathrm{ng} / \mathrm{mL}$ were $90.3 \% / 92.8 \%, 64.5 \% / 99.4 \%$ and $38.7 \% / 100 \%$.

Conclusions: AFP is a useful screening marker in normal ALT levels especially in the era of antiviral therapy where liver inflammation can be effectively controlled.

\section{PO-16 \\ Comparison of Surgical Outcome Between Minimally Invasive Liver Resection and Conventional Open Liver Resection for the Treatment of Hepatocellular Carcinoma: A Propensity-Score Matched Analysis \\ Dai Hoon Han, Eun Jung Park, Gi Hong Choi, Jin Sub Choi \\ Yonsei University College of Medicine, Seoul, Korea}

Background: This study aimed to analyze operative and survival outcomes of minimally invasive liver resection (MILR) versus conventional open liver resection (COLR) for the treatment of hepatocellular carcinoma (HCC). Moreover, we attempted to reveal the role of the robotic system in MILR (HCC).

Methods: From January 1996 to December 2012, 1014 consecutive patients underwent curative liver resection of HCC. Among these patients, 90 patients with MILR were matched to 360 patients with COLR by one-to-four propensityscore matched analysis. A multivariable logistic model based on age, gender, etiology of HCC, tumor size, multiplicity of tumor, the presence or absence of liver cirrhosis and extent of liver resection was used to estimate propensity score. Perioperative surgical outcomes and long-term survival were compared between two groups.

Results: The amount of blood loss during operation, transfusion rate and postoperative complication rate were significantly lower in MILR groups. Mean length of hospital stay after operation was significantly shorter in MILR group ( 8.57 vs. 13.44 days, $p<0.001$ ). There were 7 cases of open conversion from MILR and all cases were laparoscopic attempted liver resections. In MILR group, most of major resections were performed with robotic system $(\mathrm{n}=10, \mathrm{p}<$ 0.001). Anatomic liver resections were performed for 15 of 16 patients using robotic system. There was no difference in primary recur site between two groups. The 1-, 2-, 3-year disease-free survival rate of MILR were $84.7 \%, 66.8 \%$, and $59.6 \%$ respectively, which were comparable to those of COLR ( $\mathrm{p}=0.579$ ).

Conclusions: MILR showed better perioperative outcomes with comparable oncologic outcomes for the treatment of HCC. According to the complexity of procedures, the robotic surgery may expand the indication of minimally invasive liver resection in patients with HCC. 


\section{PO-17 \\ Laparoscopic Liver Resection for T1 and T2 Stage Hepatocellular Carcinoma Versus Open Hepatectomy - A Propensity-Score Analysis \\ Choon Hyuck David Kwon, Sanghyun Song, Jong Man Kim, Tae-Suk Kim, Hyung Hwan Moon, Sang Hoon Lee, Jae-Won Joh \\ Department of Surgery, Samsung Medical Center, Sungkyunkwan University School of Medicine, Seoul, Korea}

Background: To compare the perioperative results and survival rate of laparoscopic hepatectomy (LH) with conventional open hepatectomy $(\mathrm{OH})$ for the treatment of stage 1 and 2 hepatocellular carcinoma (HCC) using propensity score matching analysis.

Methods: From January 2008 to February 2011, 84 consecutive LH and $677 \mathrm{OH}$ were performed for HCC. After excluding TNM stage 3, tumors located in segment 1,7 , and 8 , and hepatectomies with simultaneous intraoperative radiofrequency ablation, there were a total of $74 \mathrm{LH}$ cases and 377 $\mathrm{OH}$. To exclude selection bias, we performed propensity score matching according to TNM stage, microvascular invasion, Edmondson grade, tumor size and operation range (resection for less or more than 2 segments). To get a 1:2 match, $148 \mathrm{OH}$ were selected for comparative analysis.

Results: Preoperative patient and tumor characteristics were not different between groups including cirrhosis. There were no difference in estimated blood loss and transfusion rate between groups but the operation time was longer in the LH group ( $238 \pm 109$ vs. $209 \pm 71, \mathrm{p}=0.04$ ). Resection margin was larger in LH than in $\mathrm{OH}(19.2 \mathrm{~mm}$ vs. $11.6 \mathrm{~mm}, \mathrm{p}<0.001)$. LH had shorter hospital stay (9.2 days vs 12.7 days, $p=0.007$ ) and a lower postoperative morbidity rate ( $9.5 \%$ vs. $22.3 \%, \mathrm{p}=$ 0.019). There were no operative death in $\mathrm{LH}$ and 1 in $\mathrm{OH}$ group. The disease free survival and overall survival at 4 years were not different between groups (LH $51.1 \%$ vs. OH 54.0\%, $\mathrm{p}=0.407$ and $84.5 \%$ vs. $88.6 \%, \mathrm{p}=0.502$ respectively).

Conclusions: $\mathrm{LH}$ is a feasible and safe procedure with a lower postoperative morbidity and shorter hospital stay. The disease free survival and patient survival is also comparable. Laparoscopic approach may be used an alternative in selected cases of T1 and T2 stage hepatocellular carcinoma even in the presence of cirrhosis.

\section{PO-18 \\ Single-Port Laparoscopic Liver Resection for Hepatocellular Carcinoma}

Young Kyoung You, Ho Joong Choi, Gun Hyung Na, Jae Hyun Han, Eun Young Kim, Soo Ho Lee, Tae Ho Hong, Young Chul Yoon, Sey Jun Kim, Dong Goo Kim

HBP Surgery, Seoul St. Mary's Hospital, the Catholic University of Korea, Seoul, Korea

Background: Single-port laparoscopic liver surgery has been performed sporadically. The aim of this study is to assess our experience with single-port laparoscopic liver resection as one of the usual treatment modality for hepatocellular carcinoma.

Methods: Between Mar 2009 and Dec, 43 patients underwent single-port laparoscopic liver resection for hepatocellular carcinoma. Of these, 16 were segmentectomies, 6 were left lateral sectionecties, 2 were right hepatectomies, 1 was a left hepatectomy, and 19 were nonanatomical resections.

Results: Median operating time and blood loss were 157 minutes (range 47-545 minutes) and $500 \mathrm{ml}$ (range 50-2500 $\mathrm{ml}$ ), respectively. Two procedures were converted multiport laparoscopy due to instruments length limitation and nine were converted to open liver resection. There were no major perioperative complications in these cases.

Conclusions: Single-port laparoscopic liver resection seems to be a feasible approach for hepatocellular carcinoma in selected cases.

\section{PO-19}

Dual Treatment of Reductive Surgery and Sequential Percutaneous Isolated Hepatic Perfusion for Multiple Bilobar Hepatocellular Carcinoma

Masatoshi Kudo, Takumi Fukimoto, Atsushi Takebo, Motofumi Tanaka, Hisoka Kinoshita, Kaori Kuramitsu, Shohei Kamatsu, Kenji Fukushima, Takeshi Urade, Shinichi So, Yonso Ku

Kobe University, Graduate School of Medical Sciences, Kobe, Japan

Background:Wehave done the dual treatmentcombining reductive surgery plus percutaneous isolated hepatic perfusion (PIHP) for multiple bilobar HCC since 1992. The aim of this study was to evaluate the feasibility, risks, and longterm results of Dual treatment combining reductive surgery plus PIHP as a means to improve prognosis of multiple bilobar HCC with major PVTT which were previously deemed to have no implications for surgical strategy. 
Patients and Methods: Between January, 1992, and December, 2012, 95 patients were scheduled to undergo Dual treatmentowing to the multiple bilobar HCC at Kobe University Hospital. No patients were candidates for surgical resection with curative intent or local ablative therapies because of the number, size, or bilobar diffusion of liver tumors. $49(52 \%)$ of 95 patients had the major PVTT. In patients with PVTT extending to the second or more distal portal branches in the remnant liver, they were extracted by the open method after the corresponding portal pedicles were exposed during hepatic parenchymal division. The majority of the patients had doxorubicin $\left(100 \mathrm{mg} / \mathrm{m}^{2}\right.$ to $\left.120 \mathrm{mg} / \mathrm{m}^{2}\right)$ and mitomycin C $\left(30-50 \mathrm{mg} / \mathrm{m}^{2}\right)$ as a single agent used for PIHP.

Results: We could complete Dual treatment for 85 patients, but 10 patients were failed. The 1-, 3-, 5-, year survival rates of the 85 patients are $73 \%, 31 \%, 24 \%$, respectively, with a median survival time of 20 months. And more we could complete Dual treatment for 49 patients with major portal invasion. The 1-, 3-, 5-, year survival rates of the 49 patients are $68 \%, 22 \%, 17 \%$, respectively, with a median survival time of 16 months. Uni- or multivariate analysis of factors affecting time to death of patients who underwent Dual treatment showed that the following no variates were preoperative independent factors. But normalization of PIVKA-II levels after Dual treatment is only a suitable predictable factor of prognosis in patients.

Conclusions: If the tumor number is more than four, TACE is recommended in the BCLC Group Criteria. And in the case of major PVTT, Sorafenib is recommended. But actually both treatments are palliative. Dual treatment could offer mild-term survival in a subset of patients with major portal invasion who were previously deemed to have dismal prognosis. However, our strategy yielded much longer survival term than any other previous reports. Dual treatment should be considered as a first line treatment.

\section{PO-20 \\ Treatment Strategy for Recurrent HCC}

Shinichi So, Masahiro Kido, Takumi Fukumoto, Atsushi Takebe, Motofumi Tanaka, Hisoka Kinoshita, Kaori Kuramitsu, Shohei Komatsu, Kenji Fukushima, Takeshi Urade, Yonson Ku

Kobe University Graduate School of Medical Sciences, Kobe, Japan

Background: Tumor recurrence after resection of hepatocellular carcinoma (HCC) is not rare. Especially, intrahepatic recurrence is most common. And HCC can spread from the liver to other organs, most commonly the lungs, but also bone, intra-abdominal lymph nodes, adrenal gland. But there is nothing established as treatment strategy for recurrent HCC.

Methods: A retrospective analysis was done for 416 HCC patients treated with resection between January 2003 and
December 2012 at our facilities. Fifty six re-hepatectomies and 16 extrahepatic resections were performed during this period. Firstly, we compared the efficacy of re-hepatectomy with transcatheter arterial chemoembolization (TACE) for recurrent HCC after hepatectomy. Secondary, we evaluate our algorithm of treatment for reccurent HCC.

Results: There is no significant difference in the liver function between re-resection and TACE for recurrent HCC after hepatectomy. The 1-, 3-, and 5-year overall survival rates after re-resection were $100 \%, 81 \%$, and $73 \%$.The 1-, 3-, and 5 -year overall survival rates after TACE for recurrent HCC after hepatectomy were $78 \%, 47 \%$, and $38 \%$.The 1-, 3-, and 5 -year overall survival rates after resection of extrahepatic metastasis were 68\%, 19\%, and 10\%.

Conclusions: Re-hepatectomy achieved favourable survival outcomes in the treatment of intrahepatic recurrence after hepatectomy. Early first intrahepatic recurrence after hepatecomy is risk factor of second intrahepatic recurrence after re-hepatectomy.

\section{PO-21 \\ Small for Size Living Donor Graft Showed Higher Incidence of Hepatocellular Carcinoma Recurrence Than Whole Deceased Donor Graft}

M. Park, Y. Choi, K. Lee, S. Suh, T. You, H. Kim, G. Hong, N. Yi, K. Suh

Seoul National University College of Medicine, Seoul, Korea

In regions with a limited deceased donor pool, Living donor liver transplantation (LDLT) has played an important role in treating the patients with hepatocellular carcinoma. However, LDLT may have a possible risk of hepatocellular carcinoma (HCC) recurrence compared to deceased donor liver transplantation (DDLT) due to the early graft regeneration under small for size graft. In our study, we compared HCC recurrence after LDLT and DDLT and evaluated the effect of graft recipient weight ratio (GRWR) on the recurrence of HCC after liver transplantation. 216 patients (166 LDLTs and 50 DDLTs) that underwent liver transplantation for HCC within University of California at San Francisco (UCSF) criteria from February 1999 to October 2010 were retrospectively reviewed. The patients were divided into three groups: living donor liver graft with low GRWR $(<1.0, n=59)$, living donor liver graft with high GRWR $(\geq 1.0, n=107)$ and whole deceased liver graft $(n=50)$ and three groups were similar with regards to most clinical parameters. There was a worse recurrence free survival in those patients who underwent LDLT compared with those who underwent DDLT, significantly ( $p=0.044)$. Especially, recurrence free survival rate was $86.4 \%$ and $84.7 \%$ at 1 and 3 years, respectively, for living donor liver graft with low GRWR; $94.0 \%$ and $94.0 \%$ for whole deceased liver graft 
( $\mathrm{p}=0.041)$. Multivariate analysis reveal that small graft (GRWR < 1.0) in LDLT was an independent risk factor for HCC recurrence $(p=0.022)$. Small living donor liver graft (GRWR < 1.0) increase the risk of post transplantation HCC recurrence compared to whole deceased liver graft and this could be significant for living donor liver transplantation strategies for HCC.

\section{PO-22}

\section{A Novel Protocol for ABO Incompatible Living Donor Liver Transplantation Without Graft Local Infusion and Splenectomy}

\author{
Seung Duk Lee, Young-Kyu Kim
}

National Cancer Center, Republic of Korea

Background: Graft local infusion and splenectomy have been established as pivotal strategies in $\mathrm{ABO}$ incompatible (ABO-I) living donor liver transplantation (LDLT). However, these procedures are associated with high rates of intraoperative and postoperative complications.

Methods: From January 2012 to January 2013, 13 consecutive ABO-I LDLT patients were identified at National Cancer Center, Republic of Korea. Our protocol involved rituximab $(300 \mathrm{mg} / \mathrm{m} 2)$ at preoperative 2 weeks, followed by plasma exchange (target before LDLT: isoagglutinin titer $\leq$ 1:8), basiliximab (20 mg on operation day and postoperative day 4$)$, and intravenous immune globulin $(0.8 \mathrm{~g} /$ day at postoperative day 1 and 4 ) without graft local infusion and splenectomy.

Results: The 13 patients (10 males, three females) who underwent transplantation comprised liver cirrhosis $(n=3)$ and hepatocellular carcinoma $(\mathrm{n}=10)$. The median isoagglutinin antibody titer before plasma exchange was 1:32 (range, $1: 4-1: 256)$. All patients are alive without graft failure. There was no hyperacute rejection and antibody-mediated rejection. Mean duration of hospital stay was 13.2 days. There was no recurrence of hepatitis $B$ virus ( $0 / 10$ patients), but recurrence of hepatitis $C$ virus (1/1 patient) and one positive CMV antigenemia (1/13 patients) after transplantation. No bacterial and fungal infections were observed. Complications included herpes zoster viral infection in one patient, postoperative bleeding in one patient and extrahepatic biliary stricture in three patients.

Conclusions: The new simplified ABO-I LDLT protocol using rituximab, plasma exchange, basiliximab, and intravenous immune globulin without graft local infusion and splenectomy showed good graft outcomes without hyperacute, antibody mediated rejection, and serious infection.

\section{PO-23 \\ Predictive Value of Preoperative Serum Alpha-Fetoprotein and Des-Gamma-Carboxy Prothrombin Levels on Hepatocellular Carcinoma Recurrence after Liver Transplantation

\author{
Hyung Soon Lee, JuHan Lee, Gi Hong Choi, \\ Dae Ryong Kang, Dong Jin Joo, Myoung Soo Kim, \\ Jin Sub Choi, Soon Il Kim
} \\ Department of Surgery, Yonsei University College of Medicine, Seoul, Korea}

Background: Alpha-fetoprotein (AFP) and des-gammacarboxy prothrombin (DCP) may serve as complementary markers for hepatocellular carcinoma (HCC). This study investigated whether the combined use of pre-transplant serum AFP and DCP levels improve predictive performance of the Milan criteria (MC) for HCC recurrence after liver transplantation (LT).

Methods: We analyzed 162 patients that underwent LT for HCC between 2001 and 2012. We design the MC based predictive model for post-transplant HCC recurrence. This model incorporated pre-transplant serum AFP and DCP levels: Model 1 is base model incorporated pre-transplant AFP, model 2 is base model incorporated pre-transplant DCP and model 3 is base model incorporated pre-transplant AFP and DCP. The time-dependent receiver operating characteristics (ROC) curve analysis was used to compare predictive performance for recurrence between each model over the entire range of follow-up time. To identify prognostic factors for HCC recurrence, clinicopathologic variables were assessed by Coxregression analysis.

Results: At a median follow-up of 23.5 months, 17 (10.5\%) patients developed pos-transplant HCC recurrence. In the time-dependent ROC curve analysis, predictive performance of model 1 and model 2 show no significant difference between the base model. However, model 3 show significantly better predictive performance than base model (estimated difference $=-0.069,95 \%$ confidence interval $=-0.159$ to -0.007). And pre-transplant AFP of $140 \mathrm{ng} / \mathrm{mL}$ and DCP of 65 $\mathrm{mAU} / \mathrm{mL}$ were taken as a cut-off value in the time-dependent ROC curve analysis. And multivariate analysis showed AFP $>140 \mathrm{ng} / \mathrm{mL}$ and/or DCP > $65 \mathrm{mAU} / \mathrm{mL}$ and the presence of satellite nodule were associated with post-transplant recurrence.

Conclusion: Combined use of pre-transplant AFP and DCP levels are improved predictive performance of the MC. Thus, LT candidates with HCC can be assessed more accurately by adding pre-transplant AFP and DCP levels to the MC. 


\section{PO-24 \\ Impact of Graft Regeneration to the Recurrence of Hepatocellular Carcinoma After Living Donor Liver Transplantation}

\author{
Dong Jin Joo, Su Hyung Lee, Myoung Soo Kim \\ Dai Hoon Han, Jung Jun Lee, Gi Hong Choi, \\ Jin Sub Choi, Soon Il Kim
}

Department of Surgery, Research Institute for Transplantation, Yonsei University College of Medicine, Seoul, Korea

Background: It has been suggested that tumor recurrence after resection for hepatocellular carcinoma (HCC) was related with remnant liver regeneration. In living donor liver transplantation (LDLT), graft liver usually experience regeneration and this may stimulate the growth of microscopic HCC tumors. The aim of this study was to assess the changes in graft volumes after LDLT and its relations with the recurrence of HCC.

Methods: A total 141 liver transplantations were performed for patients with HCC. Of them, deceased donor and unavailable data were excluded. All patients were measured preoperative and postoperative at 1 week and 4 weeks graft liver volume by volumetric computed tomography (CT). The study patients were divided into two subgroups according to median value of graft-recipient weight ratio (GRWR) and increasing rate of graft liver at postoperative 1 week.

Results: Of the 87 recipients, 75 recipients had no HCC but 12 experienced recurrence. Increasing rate of graft liver at postoperative 1 week and 4 weeks were significantly higher in lower GRWR group than those in higher GRWR group ( $\mathrm{P}=$ 0.003 and $P<0.001$ ), respectively (Table 1 ). Demographics, clinical variables and CT volumetry, GRWR of the study population are described in Table 2. There were no differences in preoperative GRWR and increasing rate of graft liver at postoperative 1 week and 4 weeks between the two groups. Recurred HCC patients showed more 'beyond Milan criteria' and higher tumor markers than non-recurred patients $(\mathrm{P}<$ 0.005).

Conclusions: This study showed that the lower GRWR group has higher graft regeneration rate than those in higher GRWR group. However, this study could not find significant relation between the recurrence of HCC and graft regeneration.

\section{PO-25 \\ The Utility of ${ }^{99 m}$ Tc-GSA Scintigraphy on Preoperative Evaluation of Liver Function for Major Hepatectomy \\ Motofumi Tanaka, Takumi Fukumoto, Masahiro Kido, Atsushi Takebe, Kaori Kuramitsu, Hisoka Kinoshita, Shohei Komatsu, Kenji Fukushima, Takeshi Urade, Shinichi So, Yonson Ku \\ Department of Hepato-Biliary-Pancreatic Surgery, Kobe University, Japan}

Background: The accurate evaluation of preoperative liver function is essential to avoid postoperative liver failure. In addition to conventional examination such as Child-Pugh score and indocyanine green (ICG) test, ${ }^{99 \mathrm{~m}} \mathrm{Tc}$-diethylenetriamine pentaacetic acid-galactosyl human serum albumin ( ${ }^{99 m}$ Tc-GSA) scintigraphy has been expected to be more quantitative modality. However, it still remains unclear whether this modality is helpful to decide the indication of hepatic resection. In this study, we analyzed the utility of ${ }^{99 \mathrm{~m}} \mathrm{Tc}-\mathrm{GSA}$ scintigraphy as a predictor of postoperative liver failure.

Methods: From 2005 to 2012,69 patients who underwent ${ }^{99 \mathrm{~m}} \mathrm{Tc}$-GSA scintigraphy preoperatively and right hemihepatectomy for hepatocellular carcinoma were enrolled in this study. Heart and liver ROIs were drawn manually to cover cardiac blood pool and entire liver, subsequently HH15 and LHL15 were calculated. The data of VLmg (accumulation of 99m Tc-GSA) and GSA index (LHL15/HH15) were also obtained. To estimate remnant side of liver function, remnant liver value of HH15 (rHH15), LHL15 (rLHL15), VLmg (rVLmg), and GSA index (rGSA index) were obtained by multiplying ${ }^{99 \mathrm{~m}} \mathrm{Tc}$-GSA count rate of remnant side liver and examined on the correlation with posthepatectomy liver failure (PHLF) defined by ISGLS.

Results: In this study, the mortality rate was $1.4 \%(\mathrm{n}=1)$ and the rate of PHLF and PHLF grade $B / C$ was $52 \%(n=36)$ and $36 \%(n=25)$, respectively. Whereas the value of ICG test, Child-Pugh score, and Makuuchi criteria were not associated with PHLF, The value of rLHL, rGSA index, and rVLmg were significantly associated with PHLF $(\mathrm{P}=0.003,0.004,0.006)$. When cut-off value of rLHL, rGSA index, and rVLmg were defined as $0.47,0.80$, and 0.90 by ROC analysis, these three factors were significantly associated with PHLF ( $\mathrm{P}=0.016$, $0.008,0.007)$ and PHLF grade $B / C(P=0.240,0.049,0.018)$.

Conclusions: The remnant liver function estimated by ${ }^{99 \mathrm{~m}}$ Tc-GSA scintigraphy is a good predictor of PHLF after right hemihepatectomy. 


\section{PO-26}

\section{Prediction of Recurrence After Radiofrequency Ablation for Hepatocellular Carcinoma Using Transient Elastography}

Sang Hoon Lee ${ }^{1}$, Sang Hun Lee ${ }^{1,3}$, Seung Up Kim ${ }^{1-3}$. Beom Kyung Kim ${ }^{1-3}$, Jun Yong Park ${ }^{1-3}$, Do Young Kim, Sang Hoon $\mathrm{Ahn}^{1-3}$, Kwang-Hyub Han ${ }^{1-3}$

${ }^{1}$ Department of Internal Medicine, ${ }^{2}$ Institute of Gastroenterology, Yonsei University College of Medicine, Seoul, Korea; ${ }^{3}$ Liver Cirrhosis Clinical Research Center, Seoul, Korea

Background: Liver stiffness (LS) values measured using transient elastography (TE) can accurately assess the degree of liver fibrosis. The degree of liver fibrosis is significantly associated with the risk of hepatocellular carcinoma (HCC) development. Thus, this study investigated whether LS values at radiofrequency ablation (RFA) can predict HCC recurrence after RFA.

Methods: Between May 2005 and April 2011, a total of 56 patients with HCC who underwent RFA and LS value measurement at the same day were analyzed in this retrospective study. All were followed up for monitoring the development of HCC recurrence until March 2013.

Results: The mean age of the patients ( $40 \mathrm{men}, 16$ women) was 62.1 years, and the median LS value was 23.0 $\mathrm{kPa}$. During the follow-up period (median 27.9 [range, 1.1-77.3] months), 33 (58.9\%) patients experienced HCC recurrence and 18 (32.1\%) were dead. Median time to recurrence was 17.1 (range, 2.5-67.8) months and median time to mortality was 27.9 (range, 1.1-77.3) months. Patients with recurrence showed significantly higher prevalence of liver cirrhosis, spleen size, multiple tumors, and LS values, whereas they had significantly lower platelet count than patients without recurrence (all $P<0.05$ ). On multivariate analysis, together with previous anti-HCC treatment history, patients with a higher LS value $>13.0 \mathrm{kPa}$ were at a significant greater risk for HCC recurrence after RFA with a hazard ratio of 6.981 $(P<0.05 ; 95 \%$ confidence interval, 1.650-28.782) compared with those with $\mathrm{LS}$ value $\leq 13.0 \mathrm{kPa}$. However, $\mathrm{LS}$ values were not predictive for overall survival $(P=0.237)$.

Conclusions: Our data suggest that LS values measured using TE can be a useful predictor of HCC recurrence after RFA. Further studies with large sample size are warranted for the validation of our results.

\section{PO-27 \\ Enhancement Pattern of Pretreatment Gd-EOB-DTPA-Enhanced MR Imaging Predicts Recurrence of Hepatocellular Carcinoma after Radiofrequency Ablation}

Jihyun An Ju Hyun Shim*, Seohyun Lee, Kwang-sun Lee, Danbi Lee, Kang Mo Kim, Young-suk Lim, Han Chu Lee, Young-hwa Jung, Young Sang Lee, Dong Jin Suh

Department of gastroenterology and Liver center, Asan Medical Center, University of Ulsan College of Medicine, Seoul, Republic of Korea

Background: Gd-EOB-DTPA-enhanced MR imaging is important modality for diagnosis and response evaluation after treatment of hepatocellular carcinoma (HCC) recently. This study aims to examine whether pretreatment findings in HCC using Gadoxetic Acid -enhance MRI can predict local or intrahepatic distant recurrences after radiofrequency ablation (RFA).

Methods: From 2007 to 2009, 121 consecutive patients with total 140 HCC lesions who underwent Gd-EOB-DTPAenhanced MR imaging prior to RFA were included in the study. We evaluated imaging patterns of HCC according to enhancement patterns on dynamic phase images and signal intensity on hepatobiliary-phase images. Atypical enhancement of HCC during dynamic phases was defined as iso- to hypointensity on arterial images or iso- to hyperintensity on delayed phase imagies.

Results: A total of 39 HCCs (27.9\%) demonstrated the atypical enhancement patterns on liver MR image. Iso- to hyper-intensity on hepatobiliary-phase images were observed in 11 HCCs (7.9\%). RFA for HCC was succeeded in 106 (87.6\%) patients with 125 HCC lesions (89.3\%). Of HCCs with complete ablation, 6-months, 1 -year and 2 year local recurrence rates were $6.9 \%, 9.5 \%$ and $17.1 \%$ per tumor analysis. Intrahepatic distant recurrence occurred $3.9 \%, 13.8 \%$ and $18.7 \%$ at 6-months, 1-year and 2 year per patient analysis. Two-year local recurrence rate of HCC was significantly lower in HCC with atypical enhancement patterns than those with typical enhancement patterns after RFA $(7.1 \%$ vs. $20.3 \%, P<0.05)$. However, the intrahepatic distant recurrence was not associated with enhancement patterns of HCC $(P>0.05)$. HCC with Iso- to hyper-intensity on hepatobililary-phase image was not significantly related with local and intrahepatic distant recurrences as well. $(P>0.05)$.

Conclusions: In conclusion, HCC lesions with atypical enhancement patterns on the Gd-EOB-DTPA-enhanced MR imaging are associated with radiological remission after RFA. These enhancement patterns may be a useful imaging biomarker to indicate better outcome after RFA. 


\section{PO-28 \\ Comparison of PET and Bremsstrahlung Imaging to Detect Particle Distribution in Patients Undergoing Yttrium-90 Radioembolization for Hepatocellular Carcinoma}

Siddharth A. Padia ${ }^{1}$, Adam Alessio ${ }^{2}$, Sharon W. Kwan ${ }^{1}$ David H. Lewis ${ }^{3}$, Sandeep Vaidya ${ }^{1}$, Satoshi Minoshima ${ }^{3}$

${ }^{1}$ Interventional Radiology, ${ }^{2}$ Radiology, ${ }^{3}$ Nuclear Medicine, University of Washington, Seattle, Washington, USA

Background: Currently, Bremsstrahlung SPECT is performed after patients undergo yttrium-90 $\left({ }^{90} \mathrm{Y}\right)$ microsphere radioembolization. This study assessed the value of $\mathrm{PET} / \mathrm{CT}$ imaging utilizing the inherent pair production by ${ }^{90} \mathrm{Y}$ in order to assess particle uptake.

Methods: In this prospective study, patients with large $(>5 \mathrm{~cm})$ hepatocellular carcinoma and/or tumor-associated portal vein thrombosis (PVT) were eligible to participate. Following radioembolization for hepatocellular carcinoma, patients underwent Bremsstrahlung SPECT/CT and time-offlight PET/CT imaging of ${ }^{90} \mathrm{Y}$ without additional tracer administration. Follow-up imaging and toxicity was analyzed. Imaging analyses of PET/CT and Bremsstrahlung SPECT/CT were independently performed.

Results: Thirteen patients enrolled in the study, including seven with PVT. Median tumor diameter was $7 \mathrm{~cm}$. PET/CT demonstrated precise localization of ${ }^{90} \mathrm{Y}$ particles in the liver, with specific patterns of uptake in large tumors. In cases of PVT, PET/CT showed activity within the portal vein thrombus. When correlated to short-term follow up imaging, areas of necrosis correlated with regions of uptake seen on PET/CT. Compared to Bremsstrahlung imaging, PET/CT demonstrated at least comparable spatial resolution with less scatter. Quantitative uptake in non-treated regions of interest showed significantly reduced scatter with PET/CT versus SPECT/CT at $1 \%$ versus $14 \%$, respectively $(p<0.001)$.

Conclusion: Evaluation of ${ }^{90} \mathrm{Y}$ particle uptake with PET/ CT potentially demonstrates high spatial resolution and low scatter compared to Bremsstrahlung SPECT/CT. Confirmation of particles within portal vein thrombus on PET/CT correlates with response on follow-up imaging, and may account for radioembolization's efficacy in patients with PVT.

\section{PO-29 \\ Comparison of Retreatment Outcome Between Operation/RFA and TACE According to Microvascular Invasion for Recurred Tumor After Curative Resection for Hepatocellular Carcinoma \\ Young-Joo Jin ${ }^{1}$, Jin-Woo Lee ${ }^{1 *}$, Hyun Jung Chung ${ }^{1}$, Young soo Kim ${ }^{1}$, Soon Gu Cho², Yong Sun Jeon ${ }^{2}$ \\ ${ }^{1}$ Department of Internal Medicine, Inha University Hospital, Inha University School of Medicine, Incheon, Republic of Korea; ${ }^{2}$ Department of Radiology, Inha University Hospital, Inha University School of Medicine, Incheon, Republic of Korea}

Background: Microvascular invasion (MVI) is a wellknown prognostic factor of postoperative recurrence or overall survival (OS) in patients with hepatocellular carcinoma (HCC). We compared the treatment outcome between operation/radiofrequency ablation (RFA) and transarterial chemoembolization (TACE) for recurred HCC(s) that were presented as Barcelona Clinical Liver Cancer (BCLC) stage 0 or A according to MVI after curative resection for HCC(s).

Methods: A consecutive 68 patients who diagnosed as recurred HCC(s) with BCLC stage 0 or A at our institution between 1998 and 2011 were retrospectively enrolled. We compared post-retreatment outcome between patients with operation/RFA and TACE.

Results: The median age was 53 years (range, 31-77 years). The most common cause of HCC was hepatitis B virus infection (82.4\%). Median tumor size was $1.5 \mathrm{~cm}$ (range, 1-10 $\mathrm{cm})$, and 67 (98.5\%) had HCCs within Milan criteria. The median post-retreatment follow-op duration was 27 month (range, 1-109 month). Of the 68 patients, 19 (27.9\%) underwent surgery/RFA, 47 (69.1\%) TACE, and 2 (2.9\%) lost follow-up. TACE showed significantly higher post-treatment OS and recurrence free survival rates than operation/RFA in MVI-positive patients $(P=0.03$ and $P=0.04$, respectively), unlike in MVI-negative patients $(P=0.95$ and $P=0.98$, respectively). Especially in patients with early recurrence, TACE showed significantly higher OS rate than operation/RFA in MVI-positive patients $(P=0.04)$.

Conclusions: TACE may be more effective treatment option for recurred HCC(s) with BCLC stage 0 or A than operation/RFA in MVI-positive patients, especially in those with early recurrence after curative resection. 


\section{PO-30 \\ Survival Analysis of High Intensity Focused Ultrasound Ablation Versus Transarterial Chemoembolisation for Unresectable Hepatocellular Carcinoma Larger Than $3 \mathrm{~cm}$}

Cheung Tan To, Ronnie TP Poon, Thomas Yau, Sheung Tat Fan, Chung Mau Lo

The University of Hong Kong, Queen Mary Hospital, Hong Kong

Introduction: Treatment for hepatocellular carcinoma (HCC) in the present of liver cirrhosis is always difficult. The 2 year survival of TACE is less than $30 \%$. High-intensity focused ultrasound (HIFU) ablation is a non-invasive treatment for HCC but long term survival data is lacking for large HCC. In this study we aim to analysis the outcome for patients receiving this treatment compared to TACE. Patients and Method: From Oct 2003 to Sept 2010, 2431 patients were diagnosed HCC. Total 112 patients received HIFU treatment. 27 patients has HCC larger than $3 \mathrm{~cm} .54$ patient with matched tumour characteristic receiving TACE as primary treatment were selected for comparison.

Results: There were twenty six patients received HIFU therapy during the study period. Total Forty six tumours were ablated by this treatment entity. The median age for the patients was 69 (range 49 years-84 years). The median tumour size was $4.2 \mathrm{~cm}$ (range $3-8 \mathrm{~cm}$ ). Fifty-two patients received TACE for HCC during this period. According to RECIST criteria, $50.0 \%$ patients had complete tumour response, $7.7 \%$ patients had partial tumour response, $34.6 \%$ patients had stable disease and $7.7 \%$ patients had progressive disease in the HIFU group. In the TACE group, none of the patient had complete tumour response, $21.2 \%$ patients had partial tumour response, $63.5 \%$ patients had stable disease and $15.4 \%$ patients had progressive disease. $(\mathrm{p}<0.0001)$ In the HIFU group, the one year survival was $84.6 \%$, the three year survival was $49.2 \%$ and the five year survival was $32.3 \%$. In the TACE group, the one year survival was $69.2 \%$ the three year survival was $29.8 \%$ and the five year survival was $2.3 \%$. $(\mathrm{p}=0.001)$

Conclusion: High intensity focused ultrasound ablation is a safe and effective method for unresectable HCC. The survival benefit is observed over TACE alone in selected patient.

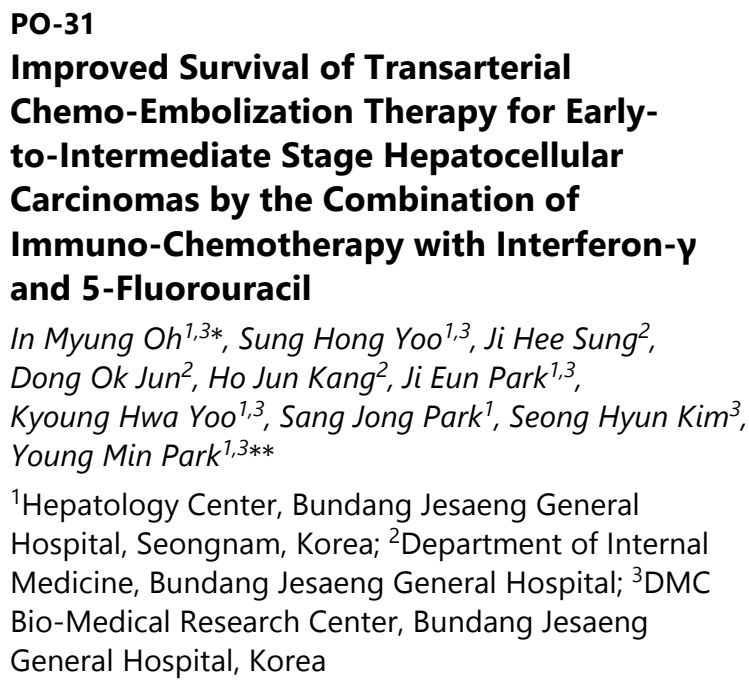

Background: Multi-modal approach improving efficacy of transarterial chemoembolization (TACE) like RFA became an important issue, especially for early-/intermediate-stage hepatocellular carcinoma (HCC). Previously, we reported that interferon- $\gamma$ (IFN- $\gamma$ ) is synergistic to anti-cancer effect of 5-fluorouracil (5-FU), and in few cases that percutaneous immuno-chemotherapy (PIC) using IFN- $\gamma$ and 5-FU was helpful for the treatment of advanced HCC with arterio-portal shunt.

Methods: In vitro, we studied the changes in apoptosisrelated genes in response to an anti-cancer cocktail of IFN- $\gamma$ and 5-FU using two HCC cell lines, Hep3B and Huh7. We performed a pilot study to evaluate therapeutic utility of PIC using a mixture of both agents to see the survival benefit of TACE for 71 HCC patients with BCLC early-to-intermediate stage, consisting of $47(66.1 \%)$ survivors with the observation period of 36 months or longer and 24 deaths, (at least two or more cycles of protocol therapy was done), compared to historical control of TACE.

Results: IFN- $\gamma$ strengthened 5-FU-induced apoptosis in Hep3B, but not in Huh7. Up-regulation of two pro-apoptotic genes, PYCARD and FASLG, was significant. Clinically, 14 of the 24 deaths died of cancer progression (10 non-cancer causes). Survival time was $140.6 \pm 97.9$ weeks in the death and $295.9 \pm$ 111.0 weeks in the survivor, respectively $(p<0.0001)$. Only the prevalence of diabetes was significant in the cancer associated deaths than in the survivors $(42.9 \%$ vs. $17.0 \%$, respectively; $p=.0436$ ), however AFP, PIVKA-II, sex, drinking, recurrence time, time-to-first recurrence, tumor number and laboratory tests were not different between the death and survivor.

Conclusions: In HCC patients with early-to-intermediate BCLC stage, combination of PIC using a mixture of IFN- $\gamma$ and 5 -FU may improve the survival of the conventional TACE using cisplatin in approximately $80 \%$, while not effective in $20 \%$. Our study suggested that PIC- IFN- $\gamma / 5$-FU is a promising strategy as a combination protocol based on TACE-cisplatin. 


\section{PO-32 \\ Efficacy and Safety of Single Agent Sunitinib in Treating Sorafenib Refractory Advanced Hepatocellular Carcinoma Patients: A Prospective, Open-label, Phase II Study Thomas Yau ${ }^{1-4}$, Roland Leung ${ }^{1}$, Hilda Wong ${ }^{1}$, Joanne Chiu', Pierre Chan 5 , Vikki Tang ${ }^{7}$, Tan To Cheung ${ }^{2}$, Roberta Pang 2,3, Sheung-Tat Fan 2,3,4, Ronnie Poon ${ }^{2,3,4}$ \\ ${ }^{1}$ Department of Medicine, The University of Hong Kong, Queen Mary Hospital, Hong Kong; ${ }^{2}$ Department of Surgery, The University of Hong Kong, Queen Mary Hospital, Hong Kong; ${ }^{3}$ Centre for Cancer Research, The University of Hong Kong, Hong Kong; ${ }^{4}$ State Key Laboratory of Liver Diseases, The University of Hong Kong, Hong Kong; ${ }^{5}$ Department of Medicine, Ruttonjee Hospital, Hong Kong}

Background: This is an open label and single arm phase II study to assess the efficacy and tolerability of sunitinib for the treatment of advanced hepatocellular carcinoma (HCC) patients who failed prior sorafenib treatment for advanced HCC.

Methods: Eligible advanced HCC patients received sunitinib $37.5 \mathrm{mg}$ continuously. Response assessment using RECIST criteria was performed after every 8 weeks. Patients who achieved either partial response or stable disease would continue the treatment till disease progression or treatment related toxicities. The primary endpoint was overall survival (OS) and the secondary endpoints were tumor response rate, progression-free survival (PFS) and safety.

Results: Between October 2008 and March 2012, 42 patients were recruited in the trial. The median age was 58 years (range, 28-81). Ninety percent of patients were chronic hepatitis B carriers with underlying Child-Pugh A and B cirrhosis in $86 \%$ and $12 \%$ of the enrolled patients, respectively. Ten (24\%) patients had tumor vascular invasion and 32 (76\%) patients had extra-hepatic metastases. Four patients achieved partial response and $11(26 \%)$ patients achieved stable disease. Overall, $36 \%$ of patients derived clinical benefits from sunitinib treatment. The median PFS was 3.5 months and OS was 7.5 months. Hand-foot skin reaction (40\%), diarrhea $(21 \%)$ and malaise $(12 \%)$ were the most commonly encountered adverse events. No treatment-related death was reported.

Conclusions: Our results demonstrated clinical activity of sunitinib with manageable safety profile in treating advanced HCC patients who failed prior sorafenib treatment. It supports the concept of sequential use of antiangiogenic tyrosine kinase inhibitors in treating advanced HCC patients.

\section{PO-33 \\ Efficacy of Hepatic Arterial Infusion of Floxuridine in Advanced Hepatocellular Carcinoma Untreatable with Conventional Treatment}

\author{
Song Yee Han, Yang Hyun Back, Sung Wook Li, \\ Kung Han Kim, Hae Kung Park \\ Dong University, Busan, Korea
}

There has been no standard treatment for advanced hepatocellular carcinoma (HCC) until now. Transarterial chemoembolization (TACE) or Radiofrequency ablation (RFA) has been performed for patients with unresectable hepatocellular carcinoma to palliate symptoms and even to prolong survival. But these treatments can be limited due to potential complications in weak patients, invasion of cancer to main bile duct and vessels, possibility of hepatic failure after treatment. Floxuridine (FUDR), an active metabolite of 5-fluorouracil (5-FU), have the advantage of being rapidly metabolized in liver.

Between January 2002 and January 2012, a total of 343 cirrhotic patients with advanced hepatocellular carcinoma were enrolled and divided by 2 groups.

Group $1(\mathrm{n}=104)$ received intra-arterial infusion chemotherapy with FUDR $(0.3 \mathrm{mg} / \mathrm{kg} /$ day for 14 days) and dexamethasone was intravenously injected to prevent sclerosing cholangitis (Day 1, 4, 7, 11) via implanted chemoport. Group 2 $(\mathrm{n}=239)$ was managed with only conservative treatment.

Mean survival time was $24.4+-2.1$ months in group 1 and 6.9+-0.6 months in group 2.

Intra-arterial chemotherapy using FUDR achieved favorable results for advanced HCC patients who had cirrhosis, and it showed better survival in selected patients. This therapy may be useful as a treatment for HCC patients with advanced hepatocellular carcinoma.

\section{PO-34 \\ Results of Neoadjuvant Hepatic Arterial Infusion Chemotherapy in Inoperable HCC Patients with Child-Pugh Class A \\ Man-Ki Kim, Sung-Su Yun, Hwa-Kyung Jung, Dong-Shik Lee, Hong Jin Kim, Tae Nyun Kim, Hyeun Joo Lee \\ Department of Surgery and Internal Medicine, College of medicine, Yeungnam University, Daegu, Korea}

Background: The prognosis of HCC patients with main portal vein tumor thrombosis (PVTT) or multiple intrahepatic lesions, or both is extremely poor and many surgeons regard them inoperable disease and also not suitable for liver transplant. We tried neoadjuvant hepatic arterial infusion chemo- 
therapy (HAI) for them to improve their survival and convert operability.

Methods: Between April 2003 to march 2013, 46 inoperable HCC patients with Child-Pugh class A were treated with neoadjuvant HAI chemotherapy. HAI was performed via a port inserted through femoral artery. The patients were treated with 5-FU $\left(750 \mathrm{mg} / \mathrm{m}^{2}\right)$ and cisplatin $\left(25 \mathrm{mg} / \mathrm{m}^{2}\right)$ from days 1 to 4 . HAI was repeated every 4 weeks. We analysed the chemoresponse with RECIST (Response Evaluation Criteria In Solid Tumors) guidelines and survival rate.

Cumulative survival was calculated using the KaplanMeier method and all statistical analyses were performed with SPSS software (version 12.0; SPSS, Inc., Chicago, IL)

Results: Twelve patients who could not receive more than 2 cycles HAI chemotherapy were excluded in this study. We analysed chemoresponse rate and survival in remaining 34 patients; overall CR, PR, SD and PD were $11.8 \%$ (four), $26.5 \%$ (nine), $47.1 \%$ (sixteen) and $14.7 \%$ (five), respectively. The median survival were 10.0 months. The 6, 12, 18 and 24 -month cumulative survival rate $70.0 \%, 67.62 \%, 52.9 \%$ and $34.6 \%$, respectively

We could do liver surgery in 8 patients $(17.3 \%)$ and the median survival was 32.0 months. The $6,12,18$ and 24 -month cumulative survival rates were $87.5 \%, 65.6 \%, 65.6 \%$, and $65.6 \%$, respectively. Among of these patients, 3 patients live now and have no recurrence for 54months, 51months, 14 months, respectively. After 6 cycle, one patient who did not received surgery showed $C R$ and survived 15 months without recurrence.

Conclusions: Neoadjuvant HAI chemotherapy can be another good option to treat inoperable HCC patients with good liver function.

\section{PO-35 \\ A Phase IV, Single-Arm, Open-Label Study of Sorafenib in Taiwanese Patients with Advanced Hepatocellular Carcinoma}

Shi-Ming Lin' ${ }^{1}$ Sheng-Nan Lu' ${ }^{2}$, Ping-Tsung Chen ${ }^{3}$, Long-Bin Jeng ${ }^{4}$, Shinn-Cherng Chen ${ }^{5}$, Chi-Tan $\mathrm{Hu}^{6}$, Sien-Sing Yang ${ }^{7}$, Marie-Aude Leberre ${ }^{8}$, Xuan Liu ${ }^{9}$, Gerold Meinhardt ${ }^{10}$

${ }^{1}$ Division of Hepatology, Linkou Chang Gung Memorial Hospital, No.5, Fuhsing Street, Kuei Shan Hsiang, Taoyuan, ${ }^{2}$ Division of Hepato-Gastroenterology, Kaohsiung Chang Gung Memorial Hospital, No.123, Tapei Road, Niaosong District, Kaohsiung City 833, ${ }^{3}$ The Division of Hematology \& Oncology, Chiayi Chang Gung Memorial Hospital, No. 6, Chia-Pu Road, Sec. West, Pu-Tz City, Chia-Yi Country 613, ${ }^{4}$ Organ Transplantation Center, China Medical University Hospital, No. 2, Yuh-Der Road, Taichung City, ${ }^{5}$ Division of Hepato-biliary-pancreatic Medicine, Kaohsiung Medical University Hospital, No.100, Tzyou 1st Road,
Kaohsiung 807, 'Division of Hepato-Gastroenterology, Buddhist Tzu Chi General Hospital and Tzu Chi University, No. 707, Sec. 3, Chung Yang Road, Hualien 970, ${ }^{7}$ Liver Center, Cathay General Hospital, No.280, Renai Road, Sec. 4, Taipei City, Taiwan; ${ }^{8}$ Clinical Statistics, Bayer HealthCare Pharmaceuticals, Loos, France; ${ }^{9}$ Global Clinical Development - GD Asia, Bayer HealthCare Co. Ltd, Beijing, China; ${ }^{10} \mathrm{Global}$ Clinical Development Oncology, Bayer HealthCare Pharmaceuticals, Montville, NJ, USA

Background: Sorafenib treatment has been shown to improve overall survival in patients with advanced hepatocellular carcinoma (HCC) in both the SHARP and Sorafenib AsiaPacific (Sorafenib AP) randomized, placebo-controlled phase III trials. In response to a post-approval request, a single-arm phase IV study ("HATT") was initiated to confirm the efficacy/ safety of sorafenib in Taiwanese patients with advanced HCC.

Methods: Patients with locally advanced or metastatic HCC were enrolled at 7 centers across Taiwan. Sorafenib was administered $400 \mathrm{mg}$ bid po. The primary objective of this study was to evaluate the safety and efficacy of sorafenib and to evaluate Child-Pugh status progression in subjects with advanced HCC treated with sorafenib.

Results: A total of 151 patients (80\% male, median age 62 ) received study treatment. The majority of patients (54\%) had stage IV disease. The primary etiologies were hepatitis $\mathrm{B}$ (54\%) or hepatitis C (27\%). Patients ( $n=149 / 151)$ were ChildPugh A (CPA); $53 \%$ CP-5 and 46\% CP-6; 95\% of patients had cirrhosis. Mean daily dose was $626 \mathrm{mg}$ and median treatment duration was 4.2 months. Efficacy outcomes were as follows: median overall survival (OS) was 8.7 months; progressionfree survival 2.7 months; time to progression (TTP) 3.8 months; response rate $6.6 \%$ (partial responses only), stable disease $41 \%$, disease control rate $48 \%$. The median time for progression from CPA to CPB/C was 88 days (CI: 57, 146 days). There were no unexpected AEs reported. Drug-related AEs were reported in $89 \%$ of patients, with worst grade 1-2 and $3-4$ AEs in $42 \%$ and $48 \%$ of patients, respectively.

Conclusions: Median OS and TTP in this trial were longer than in the Sorafenib AP trial, and in line with the sorafenib arm of recent phase 3 head-to-head trials. There were no new or unexpected safety findings. Sorafenib remains the standard of care for advanced/metastatic HCC patients in Asia. 


\section{PO-36 \\ Comparison of Efficacy Between Hepatic Arterial Infusion Chemotherapy and Sorafenib in Advanced Hepatocellular Carcinoma with Portal Vein Tumor Thrombosis}

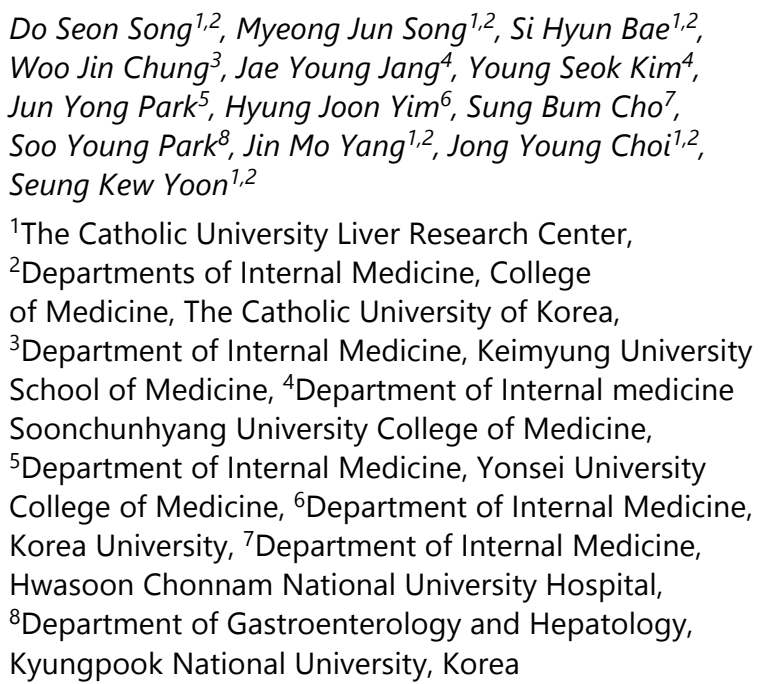

Background: Patients with hepatocellular carcinoma (HCC) with portal vein tumor thrombosis (PVTT) have an extremely poor prognosis. Although only sorafenib is recommended in advanced HCC patients in Barcelona Clinic Liver Cancer (BCLC) staging system, hepatic arterial infusion chemotherapy (HAIC) is also used in advanced HCC patients with PVTT. The objective of this study was to compare the efficacy of HAIC and sorafenib in advanced HCC patients with PVTT.

Methods: The study involved 110 advanced HCC patients with PVTT (50 in the HAIC group and 60 in the sorafenib group) observed from February 2008 to January 2013 in 7 Korean centers. All patients had good liver functions (ChildPugh score $\leq 7$ ). Primary outcomes were overall survival (OS) and time to progression (TTP). Secondary outcomes were response rates. Objective response was defined as complete response + partial response and disease control group was defined as objective response group + stable disease.

Results: Median follow-up duration was 5.6 months (range, 0.7-54.7 months). There was no significant difference in median OS between HAIC group [median 7.2 months (95\% CI 4.97-9.43 months)] and sorafenib group [median 5.7 months (95\% CI 4.45-6.96 months)] ( $p=0.150)$. Median time to progression were 3.3 months (95\% CI, 2.70-3.90) in HAIC group and 2.1 months (95\% CI, 1.52-2.68) in sorafenib group ( $p=0.034$ ). Objective response rate were $24.5 \%$ and $13.3 \%$ in HAIC group and sorafenib group, respectively ( $p=0.146$ ). Disease control rate were $91.8 \%$ and $45 \%$ in HAIC group and sorafenib group, respectively $(p<0.001)$. Tumor size $(\geq 10 \mathrm{~cm})$ (hazard ratio $2.12,95 \%$ CI 1.29-3.49) and combination with locoregional therapy (hazard ratio $0.49,95 \%$ CI $0.28-0.86$ ) were independent predictive survival factors.

Conclusion: In advanced HCC patients with PVTT, HAIC treatment can provide results comparable to sorafenib in terms of similar OS, longer TTP and higher disease control rate.

\section{PO-37 \\ Phase 2 Trial of Pexa-Vec (Pexastimogene Devacirepvec; JX-594), An Oncolytic and Immunotherapeutic Vaccinia Virus, followed by Sorafenib in Patients with Advanced Hepatocellular Carcinoma (HCC)

\author{
Jeong Heo, C. Breitbach, M. Cho, T.H. Hwang, C.W. Kim, \\ U.B. Jeon, H.Y. Woo, K.T. Yoon, J.W. Lee, J. Burke, \\ T. Hickman, K. Dubois2, L. Longpre, R. Patt, D.H. Kirn \\ Pusan National University Hospital
}

Background: Pexa-Vec is a vaccinia virus engineered to express granulocyte-macrophage colony stimulating factor (GM-CSF), thereby stimulating direct oncolysis, tumor vascular disruption and anti-tumor immunity (Nat Rev Cancer 2009). Pexa-Vec was shown to replicate in metastatic tumors following intratumoral (IT) or intravenous (IV) administration (Lancet Oncol 2008; Nature 2011). Preclinical and clinical data suggest that Pexa-Vec-induced acute vascular disruption sensitizes tumors to anti-angiogenic effects of sorafenib (Mol Ther 2011).

Methods: Treatment-refractory HCC patients received Pexa-Vec for 3 weeks (Day 1 IV, Day 8 IT and Day 22 IT) followed by sorafenib at Day 25. The primary objective of the study was to determine the safety of Pexa-Vec followed by sorafenib. Secondary objectives include disease control rate based on mRECIST and Choi (hypodensity) response criteria after Pexa-Vec only (Day 25) and after sorafenib initiation (Week 6 and 12). Optional assessments included response by positron-emission tomography (PET). Data summarized prior to database lock.

Results: Enrollment is completed: 25 patients of which 20 were refractory to sorafenib. The treatment regimen was welltolerated. Transient flu-like symptoms, including fever $(\mathrm{n}=23$; $92 \%)$, chills ( $n=19 ; 76 \%)$, headache and nausea $(n=10 ; 40 \%)$, abdominal pain and lymphopenia $(n=10 ; 40 \%)$ were the most common adverse events following Pexa-Vec. Sorafenib toxicities were consistent with the expected profile. After Pexa-Vec alone the Choi tumor response rate was $47 \%$. Following subsequent sorafenib therapy, $75 \%$ had Choi responses, including $81 \%$ of sorafenib-failure patients. The mRECIST disease control rate was $62 \%$ with Pexa-Vec alone and $59 \%$ following initiation of sorafenib. Two of 4 patients evaluable for PET response exhibited decreased PET signal after Pexa-Vec.

Conclusions: Pexa-Vec was well-tolerated and associated with Choi tumor responses and disease control in 
patients with advanced HCC. Subsequent sorafenib was well-tolerated and associated with Choi responses. Further trials of Pexa-Vec in HCC patients are warranted.

\section{PO-38 \\ Emodin Overcomes Radioresistance Induced by Hypoxia in Human Hepatoma Cells via Enhancement of Apoptosis and Inhibition of Cyclin D1}

\section{Sang Youn Hwang ${ }^{1}$, Joon Suk Kim ${ }^{1}$, Jung Woo $\mathrm{Im}^{1}$, Seon-Mi Lee ${ }^{1}$, Cheol Won Choi ${ }^{2}$, Gwang Mo Yang ${ }^{2}$, Kyu $\mathrm{Heo}^{3}$ \\ ${ }^{1}$ Department of Internal Medicine and ${ }^{2}$ Radiation Oncology, ${ }^{3}$ Research Center, Dongnam Institute of Radiological \& Medical Sciences, Korea}

Background/Aims: The application of radiotherapy of hepatocellular carcinoma (HCC) is limited due to radioresistance in tumor and radiotoxicity in nontumorous liver. Therefore, study for mechanism of radioresistance and improvement of killing effect of irradiation by therapeutic insult such as radiosensitizer etc. Emodin (1,3,8-trihydroxy6-methylanthraquinone), a family of plant derived polyphenol has been proven to have anticancer properties. There is limited data about role of emodin as radiosensitizer in human hepatoma cell line. In this study, we examined the followings: (i) whether emodin attenuated radioresitance induced by hypoxia in human hepatoma cell line, (ii) what was the mechanism of radiosensitization.

Methods: One human HCC cell line (HepG2) were used in this study. They were exposed to four different manners in environmental hypoxia (1\% 02) and normoxia; none (control), irradiation (10Gy, one fraction), emodin $(10 \mu \mathrm{M})$, and irradiation combined with emodin. Cells were then subjected to MTT assay (3-(4,5-Dimethylthiazol-2-yl)-2,5-diphenyltetrazolium bromide, a yellow tetrazole) and immunoblotting in 24 hours and 72 hours after exposure.

Results: The growth suppression of HepG2 was significantly more enhanced compared to control group in the same order as followings; combination group, emodin, and irradiation. Interestingly, radioresistance induced by hypoxia was attenuated via addition of emodin. Treatment with irradiation combined with emodin resulted in maximal upregulation of apoptotic signaling such as poly (ADP-ribose) polymerase (PARP) and caspase- 9 and downregulation of proliferation signaling such as cyclin D1.

Conclusions: Emodin overcomes radioresistance induced by hypoxia in HepG2 via enhancement of PARP, caspase- 9 and inhibition of cyclin D1. Therefore, our findings may provide new insights into understanding the pharamacological mechanism of emodin as radiosensitizer in HCC and may aid in the design of new therapeutic strategies for the radioresistant HCC induced by hypoxia.

\section{PO-39 \\ Treatment Outcome of Hepatic Re-Irradiation in Patients with HCC \\ Seung Won Seol' ${ }^{1}$, Hee Chul Park', Do Hoon Lim', Dongryul Oh', Jae Myoung Noh', Seung Woon Paik ${ }^{2}$ \\ Departments of ${ }^{1}$ Radiation Oncology and ${ }^{2}$ Internal Medicine, Samsung Medical Center, Sungkyunkwan University School of Medicine, Seoul, Korea}

Background: We evaluated the efficacy and toxicity of repeated high dose 3-dimensional conformal radiation therapy (3D-CRT) for patients with unresectable hepatocellular carcinoma (HCC), who were not eligible for further local treatments.

Methods: Between 1998 and 2011, 45 patients received hepatic re-irradiation with high dose 3D-CRT in Samsung Medical Center. After excluding two ineligible patients, 43 patients were retrospectively reviewed. RT was delivered with palliative or salvage intent, and equivalent dose of 2 Gy fractions for $\alpha / \beta=10 \mathrm{~Gy}\left(\mathrm{EQD}_{10 / 2}\right)$ ranged from $31.25 \mathrm{~Gy}_{10 / 2}$ to $93.75 \mathrm{~Gy}_{10 / 2}$ (median, $44 \mathrm{~Gy}_{10 / 2}$ ). Tumor response and toxicity were evaluated based on the modified Response Evaluation Criteria in Solid Tumors criteria and the Common Terminology Criteria for Adverse Events v3.0.

Results: The median follow-up duration was 11.2 months (range, 4.1 to 58.3 months). An objective tumor response rate was $63 \%$ with complete response in 8 lesions and partial response in 19 lesions. The tumor response rates were 81\% and $45 \%$ in patients receiving $\geq 45 \mathrm{~Gy}_{10 / 2}$ and $<45 \mathrm{~Gy}_{10 / 2}$, respectively ( $p=0.0161$ ). The median OS was 11.2 months, and it was significantly affected by the Child-Pugh class and the modified International Union Against Cancer (UICC) $\mathrm{T}$ stage. The median OS were 14.2 months vs. 6.1 months (ChildPugh Avs. B, $p<0.0001$ ) and 15.6 months vs. 8.3 months (T1-3 vs. T4, $p=0.0037)$, respectively. Higher RT dose ( $\left.\geq 45 \mathrm{~Gy}_{10 / 2}\right)$ tended to be associated with better OS (14.2 vs. 10.3 months, $p=0.1988$ ). By multivariate analysis, the Child-Pugh class and the modified UICC T stage were significant prognostic factors. Two patients developed grade $\geq 3$ toxicity, both of whom received $\geq 50 \mathrm{~Gy}_{10 / 2}$.

Conclusions: Hepatic re-irradiation is an effective and safe treatment for patients who are not eligible for further local treatment modalities, especially in selected group of patients. 


\section{PO-40 \\ Predictive Value of Vascular \\ Endothelial Growth Factor and Matrix Metalloproteinase-2 in Hepatocellular Carcinoma Patients Receiving Radiotherapy \\ Yang-Gun Suh, Eun-Jung Lee, Hyejung Cha, Seung-Hyun Yang, Jinsil Seong \\ Department of Radiation Oncology, Yonsei University College of Medicine, Seoul, Republic of Korea}

Background: Hepatocellular carcinoma (HCC) is a highly vascularized tumor, and it is known that pro-angiogenic factors such as matrix metalloproteinase (MMP)-2, MMP-9, or vascular endothelial growth factor (VEGF) involve tumor growth and metastasis. In this study, we investigated prognostic and predictive value of these pro-angiogenic factors in HCC patients receiving radiotherapy (RT).

Patients and Methods: Between September 2008 and December 2009, a total of 50 patients treated with RT were prospectively enrolled. Patients' serum and urine were collected before and $<1$ week after RT. Serum VEGF levels were analyzed using cytokine bead assay (CBA), and urine MMP-2 and MMP-9 levels were measured by enzyme-linked immunosorbent assay (ELISA). Serum VEGF levels are normalized by the patient's platelet count to eliminate VEGFscavenging effect of platelet. During RT, concurrent chemotherapy was given intraarterially in 32 and systemically in 3 patients, respectively.

Results: Tumor response was shown as complete response in $1(2 \%)$ and partial response in 34 patients (68\%). After completion of RT, serum VEGF/platelet levels were significantly increased $(\mathrm{p}=0.00)$. Patients who experienced intrahepatic tumor recurrence outside RT field showed higher pre-RT and post-RT VEGF/platelet compared with patients who did not $(p=0.05)$, whereas patients who had intrahepatic tumor recurrence inside RT field showed significantly higher pre-RT MMP-2 ( $\mathrm{p}=0.04)$. On multivariate analyses, patients with combined low pre-RT VEGF and MMP-2 levels (<median) showed a tendency for better progression-free survival (PFS) $(\mathrm{p}=0.08)$.

Conclusions: In HCC patients receiving RT, preRT level of VEGF/platelet and MMP-2 can be useful to predict treatment outcome. This study also suggests necessity of anti-angiogenic therapy, since RT might increase VEGF/platelet and higher levels of VEGF/platelet and MMP-2 are associated with intrahepatic tumor recurrence.

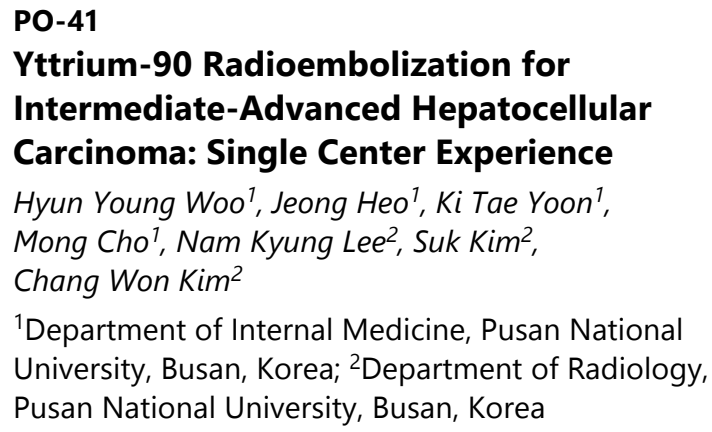

${ }^{1}$ Department of Internal Medicine, Pusan National University, Busan, Korea; ${ }^{2}$ Department of Radiology, Pusan National University, Busan, Korea

Background: Hepatocellular carcinoma (HCC) with the presence of portal vein invasion or bulky tumors were considered poor candidate for transarterial chemoemolizaion (TACE). Recent study showed that radioembolization produced better survival than conventional TACE in these patients. In this study, we investigated the safety and efficacy of radioembolization in patient with intermediated or advanced HCC.

Methods: Between September 2009 and August 2012, 25 patients were enrolled for the treatment. The response was evaluated by modified RECIST criteria and time to progression (TTP) and overall survival was estimated by the Kaplan-Meier method.

Results: Eligibility for radioembolization was $72 \%$ and 8 unsuitable due to lung shunt $>25 \%$ had larger tumor volume and more frequently associated with portal vein invasion than eligible patients. The median age was 66 years (range 50-78 years) and 14 was male. The etiology was hepatitis B in 13 and hepatitis in 1 and unknown in 4 patients. Child-Pugh score was 5 in 9, 6 in 7 and 7 in 7 patients. Median tumor size was 5 cm (range 2-19 cm). Portal vein invasion was observed in 2 patients. The median radiation dose per treatment was 1.8 GBq (range 0.17-3.93 GBq). After the first therapy, complete responses were determined in 2 patients, partial responses in 4 , stable disease 7 , and primary progression in 3 patients. 6 patient out of 10 patients without progression received additional TACE for viable portion of HCC median 106 days (range 71-106 days) after treatment whereas TTP in the others was 247 days (range 72-959 days). The median overall survival was 39.5 months (range 5.3-73.7 months). One patients experienced radiation pneumonitis and two showed liver function aggravation after treatment.

Conclusions: Radioembolization appears to have a favorable efficacy and tolerable safety profile in intermediate to advanced HCC patients. 
PO-42

Comparative Analysis of Treatment Response for Hepatocellular Carcinoma: Radioembolization Versus Hepatic Artery Infusion Chemotherapy

Hee Chul Nam, Eun Young Kim, Sung Won Lee, Jeong Won Jang, Si Hyun Bae, Jong Young Choi, Seung Kew Yoon

Department of Internal Medicine, College of Medicine, The Catholic University of Korea, Seoul, Korea

Background: Radioembolization and Hepatic arterial infusion chemotherapy (HAIC) are relatively new modalities used for the treatment of intermediate to advanced Hepatocellular carcinoma (HCC). Especially in cases with large sized tumors or portal vein tumor thrombosis (PVTT). We analyzed the treatment response after radioembolization and HAIC treatment.

Methods: A total of 69 intermediate to advanced stage HCC patients who underwent radioembolization or HAIC at Seoul St. Mary's hospital between March, 2009 and December, 2012 were analyzed (Radioembolization 19, HAIC 50). Treatment response was evaluated according to the modified Response Evaluation Criteria in Solid Tumors (mRECIST). We assessed the treatment response at 1 month and 3 months after treatment and the overall survival.

Results: In the radioembolization group, mean age was 65 years (42-78), median tumor size was $6.5 \mathrm{~cm}$ (1.5-12.7), and the PVTT was presenting in 8 of 19 patients (42.1\%). Objective response was obtained in 9 of 19 patients (47.1\%) at 3 months after the treatment and overall survival was 14.7 months (4.2-47.1). In patients with PVTT in radioembolization group, objective response was obtained in 3 of 8 patients $(37.5 \%)$ at 3 months after the treatment and overall survival was 6.9 months (4.2-18.2).

In HAIC group, mean age was 55.3 years (37-74), median tumor size was $9.05 \mathrm{~cm}(1.5-12.7)$, and the PVTT was presenting in all of the 50 patients. Objective response was obtained in 11 of 50 patients $(22.0 \%)$ at 3 months after the treatment and overall survival was 7.9 months (2.9-33.3).

There was no statistically significant difference in the treatment response and overall survival between radioembolization and HAIC groups.

Conclusions: In patients with old age and advanced stage HCC (large sized tumor and PVTT) radioembolization therapy showed relatively favorable treatment response. Further investigation in a larger number of patients would be necessary to evaluate efficacy of radioembolization therapy and for better patient selection.

\section{PO-43 \\ Is the Liver Vasculature Guided-image Fusion of Simulation CT with MRI Effective to Target Delineation of Radiotherapy for Hepatocellular Carcinoma?}

Won Sup Yoon ${ }^{1}$, Nam Kwon Lee ${ }^{1}$, Jung Ae Lee ${ }^{7}$, Dae Sik Yang ${ }^{1}$, Chul Yong Kim ${ }^{1}$, Hyung Joon Yim², Suk Keu Yeom ${ }^{3}$, Seung Hwa Lee ${ }^{3}$, Hwan Hoon Chung ${ }^{3}$, Sang Hoon $\mathrm{Cha}^{3}$

Department of ${ }^{1}$ Radiation Oncology, ${ }^{2}$ Internal Medicine and ${ }^{3}$ Radiology, College of Medicine, Korea University, Seoul, Republic of Korea

Background: To make a consistent target delineation of radiotherapy for hepatocellular carcinoma (HCC), our study evaluated a fusion method between initial diagnostic MRI with simulation CT.

Methods: Total 15 simulations were evaluated. A simulation CT was performed within 4 weeks after taking initial MRI. The thickness of simulation CT with free breathing was $2.0 \mathrm{~mm}$ and MRI with inhalation was $2.7 \mathrm{~mm}$. The acquired images on arterial phase were used. Six points from portal vein, hepatic vein and celiac axis of each simulation CT and MRI were matched by a registration program built in Eclipse $\mathrm{e}^{\mathrm{TM}}$ treatment planning system. A maximal point error along rightleft (RL), anterior-posterior (AP) and superior-inferior (SI) axes between the fused images was measured. The liver and HCC on were delineated on each simulation CT and MRI. For the indefinite HCC on simulation CT, a $5 \mathrm{~cm}$ sized virtual sphere based on the epicenter of HCC was made each simulation CT and MRI. The contours from MRI was 3-dimensinally expanded according to the maximal errors along each axis. The volume of HCC over the liver of simulation CT was edited. The expanded volume of MRI was compared with the volume of simulation CT in terms of the coverage.

Results: The median maximal errors along RL-, AP-, and SI-axes were $4 \mathrm{~mm}, 3 \mathrm{~mm}$, and $5 \mathrm{~mm}$, respectively. The median volume of liver on simulation CT and MRI were $1800.6 \mathrm{~cm}^{3}$ and $1745.4 \mathrm{~cm}^{3}$, respectively. Eights HCCs and 7 virtual masses were evaluated. The median volume of HCC on simulation CT and MRI were $66.3 \mathrm{~cm}^{3}$ and $115.3 \mathrm{~cm}^{3}$, respectively. The median uncovered volume of liver and HCC on simulation CT by MRI was 1.5\% (range 0-9.7\%) and 0.6\% (range 0-8.0\%), respectively.

Conclusion: The initial diagnostic MRI could feasibly help to assist the target delineation of HCC. 


\section{Poster Exhibition}

\section{PE-001 \\ Vascular Enhancement Patterns of HCC with Contrast-Enhanced Ultrasound}

Hae Lim Baek*, Young Kul Jung, Yun Soo Kim,

Oh Sang Kwon, Ju Hyun Kim, Duck Joo Choi,

Seung Kak Shin, Soo Young Park, Min Young Rim,

Hyeonsu Park, In Ku Yo, Seung Jun Jang,

Hyunhwa Yoon

Internal Medicine, Gachon University Gil Medical

Center, Incheon, Republic of Korea

Background: Classic enhancement pattern of hepatocellular carcinoma (HCC) with dynamic CT or MRI includes hyperenhancement in arterial phase and hypoenhancement during portal and delayed phase. It is known that vascular pattern after SonoVue 12 > injection is similar to that observed with CT or MRI. However, atypical enhancement and washout pattern are not unusually encountered with CEUS. We have studied CEUS patterns of HCC in 32 consecutive patients with HCC.

Methods: 32 HCCs were analysed (mean age 55.5, 6 females, 26 males). Twenty two were diagnosed with liver biopsy, and in 10 patients diagnosis was made with typical dynamic CT or MRI findings. CEUS using SonoVue (Bracco, Milan, Italy) were performed. Arterial, portal and delayed phases were determined as $-30 \mathrm{~s}, 31-120 \mathrm{~s}$ and $121-360 \mathrm{~s}$.

Results: HBsAg and anti-HCV were positive in $24(75 \%)$ and $5(15 \%)$ patients, respectively. Mean tumor size was $5.3 \pm$ $4.3 \mathrm{~cm}(1.1-20.0 \mathrm{~cm})$. Mean serum alpha fetoprotein was 9174 $\mathrm{ng} / \mathrm{mL}(1.3-200,000 \mathrm{ng} / \mathrm{mL})$. All the HCCs showed arterial enhancement (hyper or iso-). Arterial hyperenhancement was observed in 26(81\%) lesions, and remaining 6 (19\%) HCCs showed isoenhancement relative to the adjacent liver parenchyme. Hypoenhancement (wash-out) in portal phase was observed in only $9(28 \%)$ lesions (mean diameter: $5.1 \pm 4.2$ $\mathrm{cm})$, and remaining 23 lesions (72\%) were isodense in portal phase (mean diameter: $5.4 \pm 4.5 \mathrm{~cm}$ ). Hypoenhancement in delayed phase was observed in 28 lesions (88\%) (mean diameter: $5.8 \pm 4.4 \mathrm{~cm}$ ). And remaining 4 lesions showed persistent isoenhancement until $6 \mathrm{~min}$, and their mean diameter was $2.2 \pm 0.9 \mathrm{~cm}(\mathrm{p}=0.126)$. Enhancement during arterial phase, isoenhancement during portal and hypoenhancement during delayed phase was observed in 19 (59\%) lesions, which was most common enhancement pattern.

Conclusion: Arterial hyperenhancement with isodense portal phase and hypoenhancement in delayed phase was most common CEUS finding in HCC. It seems that tumor size affects on the hypoenhancement during delayed phase in CEUS.

\section{PE-002 \\ Proteomic Analysis for Identification of New Biomarker in Chronic Hepatitis B Related Hepatocellular Carcinoma}

Joo Ho Lee ${ }^{7}$, Ju Hyun Shim², Gi Won Song ${ }^{3}$, Mi Jung Jun', Danbi Lee ${ }^{2}$, Kang Mo Kim², Young-Suk Lim², Han Chu Lee', Young-Hwa Chung², Yung Sang Lee ${ }^{2}$, Dong Jin Suh ${ }^{4}$

${ }^{1}$ Department of Internal Medicine, CHA University of Medicine and Science; Departments of ${ }^{2}$ Gastroenterology and ${ }^{3}$ Surgery, Asan Medical Center, University of Ulsan College of Medicine; ${ }^{4}$ Department of Internal Medicine, Vievis Namuh Hospital, Seoul, Korea

Background: Hepatocellular carcinoma (HCC) is one of the leading causes of cancer-related death throughout the world. Although hepatitis B viral infection is main risk factor for HCC, the molecular mechanisms leading to HCC formation have not been clarified. To identify new biomarkers that could improve the early diagnosis in HCC of hepatitis B background, we performed a proteomic study.

Methods: In this study, we employed two-dimensional difference gel electrophoresis (2D-DIGE) combined with matrix-assisted laserdesorption ionization-time of flight (MALDI-TOF) mass spectrometry (MS) or nano flow liquid chromatography tandem MS (nanoLC-MS) to investigate differentially expressed proteins in HCC. Five patients with HCC and matched five patients with hepatitis B cirrhosis were enrolled. Cy3-labeled proteins isolated from HCC tissue were combined with Cy5-labeled proteins isolated from non-tumor tissue and separated by 2D-DIGE along with a Cy2-labeled mixture of all tumor and non-tumor samples as an internal standard. 15 protein spots were identified by MALDI-TOF or nanoLC-MS as showing significant change (paired t-test, $\mathrm{p}<0.05$ ) in the level of expression between tumor and non-tumor tissues.

Results: 15 protein spots were found significantly changed in patients with HCC. Among them, 9 proteins were successfully identified, including carbamoylphosphate synthetase I, mitochondrial ATP synthase, human liver carboxylesterase 1 , cytokeratin 9, glutamate dehydrogenase 1 , acyl-CoA dehydrogenase, S-adenosylmethionine synthetase, acetoacetyl-coenzyme A thiolase, and galactokinase. Among the aforementioned proteins, carbamoylphosphate synthetase I, human liver carboxylesterase 1, glutamate dehydrogenase 1 , galactokinase 1 , acetyl-CoA transferase and acyl-CoA dehydrogenase are proteins whose deregulation in HCC were reported at the previous studies.

Conclusions: In conclusion, proteomic study using the MALDI-TOF or nanoLC MS is an efficient strategy that enables us to identify differentially expressed proteins in HCC. Identification of potential biomarkers in HCC by MS may provide further useful insights into the pathogenesis of HCC and should be further evaluated. 


\section{PE-003 \\ Association Between Signal Transducer and Activator of Transcription1 (STAT1) Polymorphisms and Development of HCC in Korean Population}

Jae Hyuk Choi, Jaeseok Hwang*, Woo Jin Chung, Byoung Kuk Jang, Kwang Bum Cho, Kyung Sik Park, Eun Soo Kim, Dong-Hoon Shin

Division of Gastroenterology and Hepatology, Department of Internal Medicine, Keimyung University School of Medicine, Daegu, Korea

Background: Hepatocellular carcinoma (HCC) typically having a poor prognosis is the most common primary liver cancer and ranks the third most common cancer in men and sixth in women in Korea. It is the first cause of death among $50 \mathrm{~s}$ in Korea. The five-year survival rate of liver cancer is poor as $18.9 \%$. Concerning the etiological factors, $72 \%$ of patients with HCC have chronic hepatitis B (CHB), 20\% have chronic hepatitis $\mathrm{C}$ (CHC), and less than $8 \%$ have other causes including alcohol. The signal transducer and activator of transcription 1 (STAT1), a transcription factor, plays a critical role in carcinogenesis and has been implicated as a tumor suppressor. The aim of this study was to examine whether STAT1 gene polymorphisms are associated with the HCC.

Methods: We selected 5 SNPs (rs13029532, rs10199181, rs2280232, rs2280233, and rs6718902) in intron region. Five single nucleotide polymorphisms in the STAT1 gene were genotyped by direct sequencing.

Results: One hundred eighty five HCC patients and 327 control subjects were recruited. In genotype frequencies, four SNPs (rs13029532, rs10199181, rs2280232 and 2280233) showed associations with HCC $(\mathrm{P}<0.05)$. The allelic frequencies of rs10199181 were associated with HCC (P < 0.05), respectively. The allelic frequency of rs10199181 in all subjects was associated with HCC ( $p=0.003)$. The T allele frequency of rs1142636 was higher in schizophrenia (80.7\%) than that in controls (70.7\%).

Conclusions: In conclusion, our results indicate that SNPs of STAT1 may be associated with the development and of HCC in Korean population.

\section{PE-004 \\ Risk of Tumor Seeding Does Not Increase by Liver Biopsy for Hepatocellular Carcinoma Performed During Radiofrequency Ablation}

Jung Gil Park', Sun Young Ahn' ${ }^{1}$, Soo Young Park', Won Young Tak ${ }^{1}$, Young Oh Kweon ${ }^{1}$, Hyun Young Woo ${ }^{2}$, Jeong $\mathrm{Heo}^{2}$

${ }^{1}$ Division of Gastroenterology and Hepatology, Department of Internal Medicine, Kyungpook National University Hospital, Daegu, ${ }^{2}$ Department of Internal Medicine, Pusan National University Hospital, Pusan, Korea

Background: Although pathologic diagnosis of hepatocellular carcinoma (HCC) provides important information on accurate diagnosis, differentiation and prognosis of HCC, liver biopsy is not performed widely because procedure might be associated with tumor seeding during percutaneous ablative procedure. The present study aimed to assess the incidence, risk factors, and prognosis of tumor seeding after liver biopsy during radiofrequency ablation (RFA) for HCC.

Methods: From January 2009 to December 2012, 955 patients underwent treatment with RFA for HCC in Kyungpook National University Hospital. Two hundred eighty seven patients underwent percutaneous liver biopsy during RFA procedure. The biopsy was performed by coaxial technique using guiding catheter through which automated biopsy needle and RFA electrodes were passed. The risk and pattern of tumor recurrence in patients who underwent biopsy were assessed and compared to the group of patients who do not have significantly difference in tumor size and number.

Results: There were no significant differences in baseline characteristics between two groups. There were 2 cases of tumor seeding in RFA electrode tract in each group. The rate of tumor recurrence was $36.2 \%$ (104 patients) in biopsy group and 39.8\% (96 patients) in non-biopsy group ( $\mathrm{p}=$ 0.418 ). The risk of local recurrence was not significantly different between two groups $(15.7 \%$ in biopsy group vs. $13.1 \%$ in non-biopsy group, $\mathrm{p}=0.485$ ). The risk factor associated with tumor recurrence were identified as the number of tumor (OR 1.736, CI 1.148-2.625, $\mathrm{p}=0.009$ )

Conclusions: Percutaneous liver biopsy of HCC by coaxial technique does not increase the risk of tumor seeding in patients undergoing RFA. 


\section{PE-005 \\ Immunohistochemical Characteristics \\ of Combined Hepatocellular- Cholangiocarcinoma}

Jeong-Yeop Song, Soo Ji Jin, Young Seok Kim, Moon Han Choi, Yun Nah Lee, Dong Choon Kim, Hyun Jong Choi, Jong Ho Moon, Sang Gyune Kim, Se Hwan Lee, Seung Won Jeong, Jae Young Jang, Hong Su Kim, Bu Sung Kim

Department of Internal Medicine and Pathology, Soon Chun Hyang University School of Medicine, Bucheon, Korea

Background: Combined hepatocellular-cholangiocarcinoma (CHC) is relatively aggressive and rare. Despite of their unclear histogenesis, today, the diagnosis of CHC and subtypes relies on histopathological analyses and unified criteria, including molecular markers, are still pending. However, with advances in molecular biology of hepatic progenitor cell (HPC), immunohistochemical (IHC) stain plays a key role in the diagnosis of CHC. In our study, we analysed cases previously diagnosed as CHC, using IHC stains of hepatocellular, biliary/HPC markers and vimentin.

Methods: During January, 2002 and December, 2012, seventeen cases were previously diagnosed as CHC, and reevaluated in this study. And we performed IHC analysis using the following antibodies: cytokeratin (CK) 7, CK 19, c-kit, CD 56, hepatocyte paraffin (HepPar)-1, alpha-fetoprotein (AFP), carcinoembryonic antigen (CEA), carbohydrate antigen (CA) 19-9, vimentin and p53, and clinical progression was also reviewed.

Results: Among 17 cases, the number of male was15 $(88.2 \%)$ and median age was 55 . The frequency of positive finding for CK 7 was $70.6 \%(n=12)$, CK 19 was $76.5 \%(n=13)$, c-kit was $41.2 \%$ (n=7), CD 56 was $35.3 \%(n=6)$, HepPar- 1 was $64.7 \%(\mathrm{n}=11)$, AFP was $52.9 \%(\mathrm{n}=9)$, CEA was $35.3 \%(\mathrm{n}=6)$, CA $19-9$ was $76.5 \%(n=13)$, vimentin was $17.6 \%(n=3)$ and p53 was $29.4 \%(n=5)$ respectively. Underlying liver cirrhosis was noted in $35.3 \%(n=6)$ and HBV infection in $88.2 \%(n=15)$. Mean tumor number was 1.2 /case and median overall survival time was 213 days. But none of IHC results showed difference in survival.

Conclusions: Our results show that $\mathrm{CHC}$ is a neoplasm with wide histologic diversity and shows immunophenotypic expression of not only biliary markers but also HPC markers to various degrees, indicating that the histogenesis of $\mathrm{CHC}$ could be strongly associated with HPCs. But IHC results doesn't seem to predict the prognosis in this study. Further expanded studies would be needed to confirm the utility of IHC stain in CHC.

\section{PE-006 \\ Thrombocytopenia and Lower Alpha- Fetoprotein Levels are Associated with Small Hepatocellular Carcinomas \\ Yuan-Hung Kuo ${ }^{7}$, Brian I Carr ${ }^{2}$, Chih-Yun Lin ${ }^{1}$, Jing-Houng Wang ${ }^{7}$, Kwong-Ming Kee ${ }^{1}$, Sheng-Nan $\mathrm{Lu}^{1}$ \\ ${ }^{1}$ Division of Hepato-Gastroenterology, Department of Internal Medicine, Kaohsiung Chang Gung Memorial Hospital and Chang Gung University College of Medicine, Kaohsiung, Taiwan; ${ }^{2}$ Dept of Nutritional Carcinogenesis, IRCCS de Bellis, National Institute for Digestive Diseases, Castellana Grotte, Italy}

Background: Diagnosis of hepatocellular carcinoma (HCC) at early stages is important for the possibility of definite treatment and hence better survival. However, the relationship of platelet counts and alpha-fetoprotein (AFP), two common serological markers taken at diagnosis, with small HCCs remained unclear.

Aims: We aim to investigate the relationship between platelet counts and AFP with small HCCs on presentation.

Methods: A cohort of 3276 Taiwanese patients with small HCCs that were diagnosed through screening, were dichotomized by size of $<3 \mathrm{~cm}$ or $>3 \mathrm{~cm}$ maximum tumor diameter and compared for radiologic and blood test parameters, namely platelet counts and AFP.

Results: There were 2453 males and 823 females. A higher percent of females had smaller tumors. The majority of patients had single tumors. Patients with larger tumors were more likely to be complicated by portal vein thrombosis (PVT) compared to smaller tumors (35.3\% vs 3.9\%, respectively, $\mathrm{p}<$ 0.001). Significant differences between the tumor size groups included AFP values, hepatitis etiologies, levels of AST, bilirubin, albumin and platelet counts, with lower AST levels $(71.8 \pm 78.3$ vs $112.2 \pm 169.5 \mathrm{IU} / \mathrm{L}, \mathrm{p}<0.001)$, thrombocytopenia $\left(128.7 \pm 68.9\right.$ vs $\left.186.6 \pm 101.6 \times 10^{9} / \mathrm{L}, \mathrm{p}<0.001\right)$ and lower bilirubin levels $(1.4 \pm 2.2$ vs $1.9 \pm 3.1 \mathrm{mg} / \mathrm{dL}, \mathrm{p}<0.001)$ being associated with smaller tumors. When comparing patients with and without PVT, AFP levels and platelet counts were also significantly different between the 2 groups. Using the most parsimonious multinomial multiple logistic regression model of maximum tumor diameter (MTD) and portal vein thrombosis (PVT) combined, both elevated AFP (>30 ng/mL) and high platelet count $\left(>125 \times 10^{9} / \mathrm{L}\right)$ was significant for tumor size $>3 \mathrm{~cm}$ with or without PVT.

Conclusions: Smaller HCCs were associated with lower platelet counts and AFP levels. 


\section{PE-007 \\ A Hypointense HCC in Hepatobiliary Phase of Gd-DTPA-EOB-MRI Shows Pseudoglandular Pattern and Overexpression of MRP2}

\section{Kajal Mehta, Akiko Saito Satoshi Katagiri,}

Shunichi Ariismi, Yoshihito Kodera, Keiko Shiratori,

Masayuki Nakano, Masakazu Yamamoto',

Toshio Fukusato ${ }^{2}$

${ }^{1}$ Institute of Gastroenterology, Tokyo Women's Medical University, ${ }^{2}$ Department of Pathology, Teikyo University

School of Medicine, Tokyo, Japan

Background: Recently, Gd-DTPA-EOB-MRI has been frequently used for detection and diagnosis of small HCCs. The intensity of the lesions and its relation to histopathology and/ or transporter expression are reported. However, the results are still inconclusive. We report a case of hypointense HCC on hepatobiliary phase of EOB-MRI showing pseudoglandular pattern and unusual expression of OATP1B3 and MRP2.

Case: A 72 year-old asymptomatic man was detected with a small hepatic lesion on routine check-up for chronic hepatitis C. Hematological findings on admission - Albumin 3.7 g/dl, T.bil 0.5 mg/dl, AST 41 U/l, ALT 43 U/l, r-GTP 10 U/l, PT 79.4\%, ICG-R15 37\%, AFP 3523 ng/ml, AFP-L3 34.1\%, PIVKA-II 233mAU/ml, CEA $5.0 \mathrm{ng} / \mathrm{ml}$, CA19-9 $31 \mathrm{U} / \mathrm{ml}$, $\operatorname{HBsAg}(-)$, HBsAb(-), HCV-Ab(+). Conventional US showed hyperechoic heterogenous lesion. Contrast-enhanced US and CT had similar appearance with heterogenous arterial enhancement and washout on subsequent phases. EOB-MRI showed arterial enhancement and washout with hypointensity at 15 minutes- hepatobiliary phase. As these findings were compatible for HCC, we performed segmental hepatectomy. Gross specimen showed a $3-\mathrm{cm}$, green-color nodule with fibrotic capsule, so-called Green hepatoma. It was moderately-differentiated HCC with predominant pseudoglandular pattern and cholestasis. Immunohistochemistry showed OATP1B3 (+), MRP2 (+), MRP3 (-), Hep1 (+), Glyp 3 (-), CK 19 $(-)$.

Discussion: HCC is mainly detected as hypo-intense lesion on hepatobiliary phase of EOB-MRI. However, some iso/hyper-intense HCC were reported and related to pseudoglandular type pathologically. The intensity of such lesions was thought to be correlated with OATP1B3 uptake but no relation to MRP2 was established. Our case is unusual, as a hypo-intense HCC had pseudoglandular pattern and cholestasis with high OATP1B3 and MRP2.

Conclusions: We suggest the hypointensity in our pseudoglandular type HCC might be due to expression of MRP2 which leads to excretion of gadolinium along with other mechanisms.

\section{PE-008 \\ Prediction of Aggressiveness in Early-Stage Hepatocellular Carcinoma for Selection of Surgical Treatment}

Suk-Won Suh, Kwang-Woong Lee, Jeong-Moo Lee, Tae You, YoungRok Choi, Nam-Joon Yi, Kyung-Suk Suh

Seoul National University College of Medicine, Seoul, Korea

Background: In early-stage hepatocellular carcinoma (eHCC), radiofrequency ablation (RFA) has been reported to show comparable outcomes with surgery. However, microsatellites can be present in eHCC, and it results in tumor recurrence after local ablation. Biologic components of tumor as poor tumor grade and microvascular invasion were known as the risk factors for microsatellite, but it was not considered in current selection criteria for RFA. Therefore we investigated the predictors of aggressiveness in eHCC to select high risk patients of recurrence after RFA who needs surgical resection.

Methods: Between July 2007 and December 2011, a total of 370 patients with newly diagnosed eHCC(3 or fewer in number and less than $3 \mathrm{~cm}$ in size) who had no history of previous treatment were evaluated. Patients were divided into 2 groups by the type of procedure, as surgical resection group, 126 patients and RFA group, 244 patients.

At first step, in surgical resection group, preoperative risk factors for tumor aggressiveness in eHCC, which represents for the risk of microsatellites, poor tumor grade (Edmonson and Steine histologic grade, 3 or 4) or microvascular invasion were investigated.

At second step, in RFA group, identified factors were validated to reveal its influence on tumor recurrence. Recurrence free survival of patients with and without the identified risk factors in both group were also compared.

Results: At, first step, in surgical resection group, the proportion of high risk group for tumor aggressiveness who had poor histologic grade or microvascular invasion was 77.8\%. Tumor size, PIVKA-II more than $40 \mathrm{nAU} / \mathrm{mL}$, and multiplied AFP and PIVKA-II (A*P) more than 1,600 were significant in univariate analysis and the only significant factor in multivariate analysis was $\mathrm{A}^{*} \mathrm{P}$ more than 1,600 (HR 3.837. 95\% CI 1.493-9.861, $\mathrm{p}=0.005$ ).

At second step, in RFA group, identified risk factor, $A^{*} \mathrm{P}$ more than 1,600 was validated in terms of HCC recurrence. The significant risk factors for tumor recurrence in RFA group were albumin (HR 0.456, 95\% CI 0.313-0.666, p = 0.000) and $\mathrm{A}^{*} \mathrm{P}$ more than 1,600 (HR 1.835, 95\% CI 1.222-2.755, $\mathrm{p}=$ 0.003).

Recurrence free survivals were analyzed after adjusting several factors and it shows significant difference in RFA group by the level of combined AFP and PIVKA (HR = 1.835, $95 \% \mathrm{CI}=1.222-2.755, \mathrm{p}=0.003$ ), but there was no significant difference in surgical resection group $(p=0.214)$. Subgroup analysis was done for single tumor in early-stage HCC. 1-year 
recurrence free survivals which represents the results of local recurrence were analyzed and among the patients with $\mathrm{A}^{*} \mathrm{P}$ more than 1,600, surgical resection group shows superior outcomes than RFA group in terms of HCC recurrence. (HR = $2.340,95 \% \mathrm{CI}=1.049-5.219, \mathrm{p}=0.038$ ) But there was no significant difference by type of procedure in patients, $A^{*} \mathrm{P}$ lower than 1,600. ( $\mathrm{p}=0.872$ ).

Conclusions: Combined AFP and PIVKA is useful predictor of aggressiveness in early-stage HCC. It predicts tumor recurrence after RFA, as well. Therefore, if the level of this predictor is high in early-stage HCC surgical resection should be considered for $1^{\text {st }}$ line therapy.

\section{PE-009 \\ Change in HBV DNA After the Liver Resection in Non-Replicating HBV Related Hepatocellular Carcinoma Patients: Impact of HBV DNA on the Treatment Outcome}

\author{
Jung Il Lee ${ }^{1}$, Ja Kyung Kim ${ }^{1}$, Jin-Woo Lee ${ }^{2}$, Hyung Jung \\ Chung', Young Soo Kim², Kwan Sik Lee ${ }^{1}$ \\ ${ }^{1}$ Department of Internal Medicine, Gangnam Severance \\ Hospital, Yonsei University College of Medicine, \\ ${ }^{2}$ Department of Internal Medicine, Inha University \\ School of Medicine, Seoul, Korea
}

Background: Sustained low hepatitis B virus (HBV) load may predict good outcome after the curative resection of hepatocellular carcinoma (HCC). Naturally, HBV level may fluctuate, and its change after the liver resection in patients with non-replicating baseline HBV DNA is not delineated clearly. This study investigated viral reactivation after the liver resection in non-replicating HBV DNA related HCC patients and its impact on the HCC treatment outcome.

Methods: Medical records of 198 patients who underwent liver resection due to HBV related HCC were retrospectively reviewed. One hundred one patients whose serum HBV DNA levels were serially checked starting from the time of liver resection were analyzed. Out of 101 patients, 49 patients had baseline HBV DNA $<2000 \mathrm{IU} / \mathrm{mL}$, and 33 patients had undetectable HBV DNA. In order to seek whether the number of patients with viral reactivation after the surgery exceeds that of HBs Ag carriers without liver resection, we evaluated HBV DNA in 33 age matched patients with nonreplicating HBV DNA at the baseline and 6 months apart.

Results: From the 33 patients with non-replicating HBV DNA who underwent liver resection, 10 patients had increased serum HBV DNA $\geq 2000 \mathrm{IU} / \mathrm{mL}$ after the liver resection. On the other hands, only 1 patient from the group without liver resection had significant HBV viremia within 6 months. Perioperative initiation of antiviral therapy was the only factor that affected postoperative HBV DNA reactivation (hazard ratio (HR): $0.015 ; 95 \%$ CI: 0.002-0.144; $P=0.000$ ).Postoperative viral reactivation (HR: 4.438; 95\% CI: 1.797-10.989; $\mathrm{P}=$
0.001), along with not normalizing AFP 3 months after the surgery (HR: 4.222; 95\% CI: 2.058-8.663; P = 0.000), existence of microvascular invasion (HR: 1.353; 95\% CI: $1.353-$ 5.297; $\mathrm{P}=0.005$ ), and experiencing major liver resection (HR: 2.058; 95\% CI: 1.209-6.103; $\mathrm{P}=0.016$ ) predicted recurrence after the curative liver resection.

Conclusions: This study shows that non-replicating HBV related HCC may also have serum HBV DNA reactivated after the surgery, and postoperative viral reactivation may predict HCC recurrence after the curative liver resection.

\section{PE-010 \\ Risk Assessment of Hepatocellular Carcinoma in Patients with Chronic HBV Infection by LSM-Spleen Diameter to Platelet Ratio Score (LSPS) \\ Seung Hwan Shin ${ }^{1 *}$, Seung Up Kim ${ }^{1-4 *}$, Jun Yong Park ${ }^{1-4}$, Do Young Kim ${ }^{1-4}$, Sang Hoon Ahn ${ }^{1-5}$, Kwang-Hyub Han ${ }^{1-5}$, Beom Kyung Kim ${ }^{1-4}$ \\ ${ }^{1}$ Department of Internal Medicine, ${ }^{2}$ Institute of Gastroenterology, ${ }^{3}$ Liver Cancer Special Clinic, Yonsei University College of Medicine, Seoul, ${ }^{4}$ Liver Cirrhosis Clinical Research Center, Seoul, ${ }^{5}$ Brain Korea 21 Project for Medical Science, Seoul, Korea}

Background: Liver stiffness is a reliable, noninvasive marker assessing not only the degree of fibrosis but also development of hepatocellular carcinoma and hepatic decompensation. Recently, LSM-based prediction model, LSM-spleen diameter to platelet ratio score (LSPS = LSM x spleen diameter/ platelet count) proved to be well correlated with development of portal hypertension-related cirrhotic complications. Here, we assessed the prognostic value of LSPS for development of HCC in patients with chronic hepatitis B virus (HBV) infection during long-term follow-up.

Methods: We retrospectively analyzed 227 patients with chronic HBV infection who had well-preserved liver function, and no history of prior decompensation or HCC from 2006 to 2008. The major end point was diagnosis of HCC, examined using Kaplan-Meier and Cox regression method.

Results: The median age was 45 years and 156 (68.7\%) were male. During follow-up (median 61 months), 18 patients were diagnosed as HCC. Patient with HCC had the higher LS values and spleen size, and the lower platelet counts (all p < 0.05). On multivariate analysis, LSPS were identified as an independent predictor of development of HCC adjusting age, albumin[hazard ratio (HR) 1.572, $\mathrm{p}=<0.001$ ] When patients were stratified into three groups (LSPS $<1.1,1.1-2.5$ and $\geq$ 2.5 ), the 5-year cumulative risk of HCC development significantly increased in association with the higher LSPS value (4.0\%, 13.8\%, 36.2\%, respectively, $\mathrm{p}<0.001)$. From multivariate analysis, patients with LSPS 1.1-2.5 [HR 3.782, $\mathrm{p}=$ 0.039 ] and patients with LSPS $\geq 2.5$ [HR 12.460, $p<0.001$ ] 
were at a great HCC development compared to patients with LSPS $<1.1$.

Conclusions: LSPS is also useful in risk assessment of developing HCC among patients with chronic HBV infection. Effort to early detect HCC and careful regular follow up are needed for patients with LSPS $>1.1$

\section{PE-011 \\ Clinicopathological Characteristics of PIK3CA and $H B x$ Mutations in Korean Patients with Hepatocellular Carcinomas}

Woo Jin Chung, Byung Kuk Jang, Jae Seok Hwang

Department of Internal Medicine, Keimyung University Dongsan Medical Center, Daegu, Korea

Background: Frequency of PIK3CA mutation was various and its clinical significance has not been studied in Korean patients with HCC. In addition, the $H B x$ gene seems to be a key modulation of cellular functions, leading to HCC. We aimed to investigate the clinical significance of PIK3CA and $H B x$ mutations in HCC patients.

Methods: HCC patients who underwent hepatectomy at Keimyung University Dongsan Hospital were enrolled in the study. Mutations in exon 9 and 20 of the PIK3CA gene and the $H B x$ gene were detected by polymerase chain reaction and direct DNA sequencing.

Results: 1.50 patients were enrolled in this study (39 male, mean age $57.9 \pm 11$.11years). 2. PIK3CA mutation of exon 20 was detected in 7 patients (14\%), however, no mutation was detected in exon 9. Two types of point mutations (H1047L and K1041G) were found in exon 20. 3. The prevalence of point mutations was positively correlated with tumor size. Median tumor sizes were $5.88 \pm 3.99$ and $9.50 \pm$ $4.60 \mathrm{~cm}$ each in the absence and presence of $P I K 3 C A$ point mutation $(P=0.049)$. 4 . The PIK3CA point mutation was positively correlated with microscopic marginal involvement (PIK3CA mutation: 5/7, Absence of PIK3CA mutation: 15/43, $\mathrm{P}=0.027)$. 5. Among 42 patients who have HBsAg 17 patients (40.5\%) had $H B x$ point mutation and 4 patients $(9.52 \%)$ had PIK3CA mutation. $H B x$ point mutation and PIK3CA mutation were positively correlated with tumor size $(H B x$ mutation: $6.54 \pm 5.00$, PIK3CA mutation: $10.72 \pm 4.81$, Absence of any mutation: $5.30 \pm 3.39 \mathrm{~cm}, \mathrm{P}=0.043$ ) and microscopic marginal involvement (HBX mutation: 9/17, PIK3CA mutation: 2/4, Absence of any mutation: $5 / 21, \mathrm{P}=0.046$ ).

Conclusions: Prevalence of PIK3CA and $H B x$ mutations were correlated with the size of HCC and microscopic marginal involvement. So when we plan to do hepatectomy for HCC patients, efforts should be made to obtain an adequate tumor microscopic safety margin in PIK3CA and $H B X$ mutated patients.

\section{PE-012 \\ Histologic Differentiation Predicts the Survival of Patients with Hepatic Resection for Hepatocellular Carcinoma}

Woong Cheul Lee ${ }^{7}$, Jae Young Jang ${ }^{1 *}$, Jin Nyoung Kim', Soung Won Jeong ${ }^{7}$, Eui Ju Park ${ }^{7}$, Byoung Moo Lee ${ }^{1}$, Sung Woo Ryu ${ }^{7}$, Ji Hye Kim', Yun Nah Lee ${ }^{3}$, Sae Hwan Lee, ${ }^{2}$ Sang Gyune Kim ${ }^{3}$, Sang-Woo Cha', Young Seok Kim ${ }^{3}$, Young Deok Cho ${ }^{1}$, Hong Soo Kim², Boo Sung Kim ${ }^{7}$

${ }^{1}$ Institute for Digestive Research, Digestive Disease Center, Department of Internal Medicine, College of Medicine, Soonchunhyang University, Seoul, ${ }^{2}$ Department of Internal Medicine, College of Medicine, Soonchunhyang University, Cheonan, South Korea;

${ }^{3}$ Department of Internal Medicine, College of Medicine, Soonchunhyang University, Bucheon, Korea

Background: In resection for hepatocellular carcinoma (HCC), patient selection depends on morphological features and liver function. In patients with HCC, we performed a clinico-pathological analysis of risk factors that affected survival after liver resection.

Methods: Sixty seven patients with liver resection for HCC were enrolled and data were collected retrospectively. Variables were age, gender, etiology, preoperative alpha-fetoprotein (AFP) levels, Child-Pugh and MELD scores, modified UICC stage, portal vein tumor invasion, and histological differentiation.

Results: Sixty seven patients were evaluated and 6 patients were excluded (Three patients with combined hepatocellular-cholangiocarcinoma, two patients because of early postoperative death without HCC recurrence and one patient accidental death without HCC recurrence). The mean age of the patients was $57.4 \pm 10.5$ years. The mean follow-up time was $51.2 \pm 34.8$ months. The cumulative 1-, 3-, 5-, and 7-year survival rates were $91.3 \%, 64.5 \%, 45.0 \%$, and $45.0 \%$, respectively. The 1-, 3-, 5-, and 7-year disease-free survival rates were $80.4 \%, 50.5 \%, 12.9 \%$, and $12.9 \%$, respectively. Multivariate analysis showed that modified UICC stage ( $p=0.005)$, portal vein tumor invasion $(p=0.024)$ and poor histological differentiation ( $p=0.012$ ) were independent factors for survival and tumor recurrence.

Conclusions: Although morphological stage and liver function are important factors of liver resection, for proper patient selection in liver resection for HCC, prognostic criteria related to tumor histology (especially histological differentiation) should be considered. Poor histological differentiation is indicators of poor prognosis after liver resection for HCC. 


\section{PE-013 \\ Liver Specific Graded Prognostic Assessment Can Predict the Outcome for Patients with Brain Metastases from Hepatocellular Carcinoma \\ Seungtaek Lim ${ }^{1}$, Soohyeon Lee ${ }^{1}$, Kwang-Hyub Han², Hyejin Choi $^{1}$ \\ Division of ${ }^{1}$ Oncology, ${ }^{2}$ Division of Gastroenterology, Department of Internal Medicine, Yonsei University \\ College of Medicine, Seoul, Korea}

Background: After the introduction of sorafenib which showed prolongation of survival in patients with advanced HCC, the incidence of brain metastasis seemed to increase. Stratifying patients by prognostic factors can be useful for treatment decision. Although diagnosis-specific Graded Prognostic Assessment (GPA) has been identified for several cancer types, optimal treatment strategy for brain metastasis from HCC has not been well established.

Methods: A total of 56 HCC patients were newly diagnosed with brain metastasis at Yonsei University Health Center between 2007 and 2011. Using SPSS program, univariate and multivariate analyses of the prognostic factors were performed. With the P value less than 0.05 as cutoff value, the significant prognostic factors were used to develop the HCC specific-GPA (HCC-GPA).

Result: The median overall survival after brain metastasis in all patients was only 5.9 weeks $(95 \%$ confidence interval: 4.8-7.4 week). Significant prognostic factors were the presence of extracranial lesion, and Child-Pugh-Class score. Using those variables, we newly developed HCC specific GPA scoring system (HCC-GPA): extracranial metastasis (Yes:0, No: 1 point), and Child-Pugh-Score (C:0, B:1, A:2 point). The median survival time from brain metastasis were significantly separated by HCC-GPA grouping ( 3.9 weeks in group 1 (HCC-GPA score 0 or $1.0, \mathrm{~N}=24$ ), 6.3 weeks in group 2 (HCC-GPA score 2.0, $\mathrm{N}=28$ ), and 94.0 weeks in group 3 (HCC-GPA score 3.0, N=4); $\mathrm{p}<0.001$ by log rank test). This prognostic index was validated in different patient cohort $(\mathrm{N}=62)$.

Conclusion: Although overall prognosis of patients with brain metastasis from HCC is dismal, the present data demonstrated that newly developed HCC-GPA score can discriminate the prognosis of HCC patients with brain metastasis. It can be used as a valuable tool to select patients who can be good candidates for active local treatment.

\section{PE-014 \\ Prognosis After liver Resection for 7th AJCC Stage I Hepatocellular Carcinoma: Reclassification According to Preoperative Tumor Markers and Platelet Counts

\author{
Dai Hoon Han, Sung Hoon Choi, Gi Hong Choi, \\ Jun Yong Park, Do Young Kim, Sang Hoon Ahn, \\ Kyung Sik Kim, Jin Sub Choi and Kwang-Hyub Han \\ Yonsei University College of Medicine, Seoul, Korea
}

Background: 7th AJCC TNM stage defines stage I hepatocellular carcinoma (HCC) as a solitary tumor without vascular invasion. However, there is the limitation of pathologic examination for entire specimen to determine the exact stage of HCC. In this study, we tried to reclassify stage I hepatocellular carcinoma according to patient-, surgery-, and tumor related prognostic factors.

Methods: From October 2000 to April 2011,872 patients underwent curative resection for HCC at Severance hospital, Yonsei University College of Medicine, Seoul, Korea. Among them, 389 patients included 7th AJCC stage I. Prognostic factors for disease-free survival were identified for 369 patients whose preoperative serum tumor markers of alphafeto protein (AFP) and protein induced by vitamin K absenceII (PIVKA II) were available.

Results: Low serum albumin $(\leq 3.5 \mathrm{~g} / \mathrm{dL})$, low platelet count $(\leq 100,000 / \mathrm{mm} 3)$, minor resection of liver and simultaneously higher preoperative serum tumor makers of AFP $(>20$ $\mathrm{IU} / \mathrm{mL}$ ) and PIVKA II ( $>40 \mathrm{mAU} / \mathrm{mL})$ were poor prognostic factors for disease-free survival after hepatectomy. Both higher serum AFP and PIVKA II level ( $p=0.026)$, and lower platelet count ( $\mathrm{p}=0.005)$ were independent worse prognostic factors for disease-free survival. 1-, 3-, 5-year disease free survival of the patients with higher platelet count and lower serum AFP or PIVKA II were significantly higher than the others. $(88.9 \%, 71.4 \%$, and $62.0 \%$ Vs $79.6 \%, 54.6 \%$, and $44.7 \%, \mathrm{p}=0.001)$

Conclusions: Stage I HCC could be reclassified into two groups according to combined serum tumor markers with AFP and PIVKA II and platelet counts. 


\section{PE-015 \\ Improved but Still Unsatisfactory Hepatocellular Carcinoma Surveillance in Korea}

Ji Young Park, Dae Myung Kim, Jin Tae Hwang, Sung In Yu, Ki Jun Jang, Dong Hyun Sinn, Tae Joo Jeon, Tae-Hoon Oh, Won Chang Shin, Won-Choong Choi

Department of Internal Medicine, Sanggye Paik

Hospital, Inje University, Seoul, Korea

Background: Surveillance of hepatocellular carcinoma (HCC) can detect tumors at an early stage and is recommended for at-risk individuals. However, whether surveillance of HCC is well-implemented in clinical practice remains unknown.

Methods: Data from the Korea National Health and Nutritional Examination Survey (KNHNES)-V, which was performed at year 2010, were analyzed. We analyzed whether at-risk individuals received HCC surveillance. Data were compared to KNHNES-II, which was performed at year 2001.

Results: The prevalence of HBsAg was $4.9 \%(291 / 6,000)$ at year 2001, which was decreased at year 2010 (3.5\%, $205 / 5,836, p<0.001)$. Individuals who responded that they received HCC surveillance among HBV carriers increased from $13.4 \%(39 / 291)$ at year 2001 to $37.6 \%(77 / 205)$ at year $2010(\mathrm{p}<0.001)$. However, at year 2010 , still only 30 carriers $(30 / 205,14.6 \%)$ HBV carriers reported that they had HCC surveillance within 6 months. Thirteen HBV carriers had HCC surveillance between 6 months - 12 months, and 34 carriers had HCC surveillance beyond 12 months. At year 2010, HCC surveillance (within 6 months) rate was 19.6\% (21/107) among individuals who responded that they are infected with hepatitis B virus (HBV), 33.3\% (3/9) among individuals who responded that they are infected with hepatitis $\mathrm{C}$ virus (HCV), and $15.8 \%(3 / 19)$ among individuals who responded that they are cirrhotic. However, among 107 individuals who responded that they are infected with $\mathrm{HBV}$, only 45 patients (42.1\%) were actually positive for HBsAg, and among 205 individuals who were positive for hepatitis B surface antigen (HBsAg), only 45 individuals (22.0\%) were aware of their HBV infection. HCC surveillance (within 6 months) rate was higher in HBV carriers aware of their HBV infection than those unaware of HBV infection (33.3\% vs. 9.4\%, p < 0.001).

Conclusions: The KNHNES-V shows that HCC surveillance in Korea is still unsatisfactory. A way to enhance adherence to HCC surveillance recommendation is urgently needed.

\section{PE-016 \\ Role of AFP and PIVKA-II as Markers for Monitoring Response and Recurrence after Resection for HCC}

Sung Won Lee ${ }^{1,2}$, Hee Chul Nam 1,2, Jeong Won Jang 1,2, Si Hyun Bae ${ }^{1,2}$, Seung Kew Yoon ${ }^{1,2}$, Jong Young Choi ${ }^{1,2}$

${ }^{1}$ The Catholic University Liver Research Center,

${ }^{2}$ Department of Internal Medicine, College of Medicine, The Catholic University of Korea, Seoul, Korea

Background: Liver resection is the treatment of choice for most cases of early stage hepatocellular carcinoma (HCC). However, the recurrence rates of HCC remains high even after curative hepatectomy. The unresolved clinical issue is how to monitor HCC after curative surgical resection. In this retrospective study, we analyzed the association between changes in AFP and PIVKA-II with the recurrence of HCC after curative hepatectomy.

Methods: A total of seventy nine consecutive HCC patients who underwent curative hepatectomy at Seoul St. Mary's hospital between September, 2010 and August, 2012 were analyzed. The recurrence rates and postoperative survival rates were compared according to the tumor characteristics and preoperative and postoperative tumor marker values.

Results: During the median follow-up periods of 24.2 months (2.3-47.5 months), HCC recurrence developed in 32 of 79 patients $(40.5 \%)$. The median time to the development of recurrence was 6.0 months. In the HCC recurrence group, the median AFP were 105, 7.45, 6.1, 16 and $5.25 \mathrm{ng} / \mathrm{ml}$ at pre-op, 1 month, 3months, 6months and 12months after hepatectomy, respectively, compared with the non-recurrence group of $6.1,4.4,3.25,2.9$ and $3 \mathrm{ng} / \mathrm{ml}$. The median PIVKA-II were 168, 19.5, 21, 27.5 and $27 \mathrm{mAU} / \mathrm{ml}$ in the recurrence group compared with the non-recurrence group of 26 , $19,16.5,17,15.5$, respectively. In multivariate analysis for the predictive markers of HCC recurrence, tumor size $>4 \mathrm{~cm}$, preoperative AFP > 20ng/ml, 1 month postoperative AFP > 20 $\mathrm{ng} / \mathrm{ml}$, and 1 month postoperative PIVKA-II $>40 \mathrm{mAU} / \mathrm{ml}$ were identified as significant factors $(p<0.05, p<0.05, p<$ 0.001 , and $p<0.05$ respectively).

Conclusions: These results suggest that AFP or PIVKA-II values that exceed their normal range when measured at one month after hepatectomy may be predictive of HCC recurrence. The patients with these risk factors should be cautiously followed up at short-term intervals after curative hepatectomy. 


\section{PE-017 \\ Alpha-Fetoprotein has a Role in Detection of Small Hepatocellular Carcinomas in Korea}

Jae-Jun Shim, Jeong Min Chae, Sung Hoon Hong, Hyuk Kim, Tae-Young Kim, Seok Ho Dong,

Byung-Ho Kim

Department of Internal Medicine, Kyung Hee University School of Medicine, Seoul, Korea

Background: The role of AFP is still controversial in surveillance of hepatocellular carcinoma (HCC). The purpose of surveillance is to detect HCC at an early stage. We investigated role of AFP in detection of very early stage of HCCs (1 2 $\mathrm{cm}$ ).

Methods: From Jan 2008 to Dec 2012, 420 HCCs were newly diagnosed using American Association for the Study of Liver Disease (AASLD) guidelines. A small HCC was defined by a single nodule $(1 \sim 2 \mathrm{~cm})$ with histologically confirmed or presenting typical enhancing pattern on dynamic computed tomography (CT) or MRI. Contribution of AFP to trigger shortterm follow up or confirmatory tests was investigated in small HCCs.

Results: Of the new HCCs, there were 56 small HCCs $(13.3 \%)$. Forty six $(82.1 \%)$ were detected by periodic or inconsistent surveillance tests, the others $(17.9 \%)$ were detected incidentally. Fifty three (94.6\%) had liver cirrhosis and $40(71.4 \%)$ had hepatitis B virus (HBV) infection. Only 3 (5.4\%) had serum alanine transaminase (ALT) $>80 \mathrm{IU} / \mathrm{L}$. Sixteen patients $(28.6 \%)$ had mildly elevated ALT (40 80 IU/L). Ultrasound (US) and CT detected small HCCs firstly in $26(46.4 \%)$ and $24(42.9 \%)$, respectively. The remaining 6 (10.7\%) patients were detected by only elevated AFP level. Among the detected small HCCs, 18 (32.1\%) patients had elevated AFP ( $\geq 20 \mathrm{ng} / \mathrm{mL}$ ).

Conclusions: About $10 \%$ of small HCCs can be detected by only AFP. Until more sensitive and cost-effective screening tools are developed, AFP should not be excluded in surveillance tests in order to detect more HCCs at the earliest stage.

\section{PE-018 \\ Clinical Characteristics of Patients with Initially Diagnosed as Advance Staged Hepatocellular Carcinoma During Surveillance At-Risk Populations}

\author{
Young-Joo Jin ${ }^{1}$, Jin-Woo Lee ${ }^{1 *}$, Hyun Jung Chung ${ }^{1}$, \\ Young soo Kim ${ }^{1}$, Soon Gu Cho², Yong Sun Jeon ${ }^{2}$ \\ ${ }^{1}$ Department of Internal Medicine, Inha University \\ Hospital, Inha University School of Medicine, \\ ${ }^{2}$ Department of Radiology, Inha University Hospital, \\ Inha University School of Medicine, Incheon, Korea
}

Background: Despite the regular surveillance for hepatocellular carcinoma (HCC), we can often meet the patients who are initially diagnosed as having advanced staged HCC. Therefore, we investigate the frequency of the patients who were initially expressed as advanced HCC during surveillance at-risk population, and intended to evaluate the clinical characteristics of these patients.

Methods: A total of 120 patients who were initially diagnosed as HCC during surveillance at our institution between January 1999 and January 2013 were consecutively enrolled. HCC surveillance was done with regular follow-up of abdominal ultrasonograpy and serum alpha-fetoprotein at-risk population. HCC was diagnosed based on the criteria of the American Association for the Study for Liver Diseases. Barcelona Clinical Liver Cancer (BCLC) staging system was used for tumor stage.

Results: Of the 120 patients, 15 (12.5\%) patients were diagnosed as having initially BCLC stage C. Of these 15 patients, the median age was 55 years (range, 37-79 years), and 15 $(88.2 \%)$ were male. All of them had clinically liver cirrhosis. Fourteen $(93.3 \%)$ patients had nodular tumor type with the median tumor size of $3.1 \mathrm{~cm}$ (range, $1.5-6.7 \mathrm{~cm}$ ). Only one patient had infiltrative tumor type. Lymph node metastasis was observed in $8(53.3 \%)$ patients, and portal vein tumor thrombosis was observed in 7 (46.7\%) patients.

Conclusions: Our data showed that $12.5 \%$ of HCC patients may express as having initially advanced HCC at-risk population, especially with liver cirrhosis despite the regular surveillance. Moreover, portal vein tumor thrombosis or lymph node metastasis may not be detected early with ultrasonography. However, large scaled prospective study needs to be performed to confirm our data. 


\section{PE-019 \\ Feasibility of Laparoscopic Re-Resection for Patients with Recurrent Hepatocellular Carcinoma \\ Albert Chan Ronnie Poon, Kenneth Chok, Tan To Cheung, Jeff Dai, See Ching Chan, Chung Mau Lo \\ Division of Hepatobiliary and Pancreatic Surgery, and Liver Transplantation, Department of Surgery, The University of Hong Kong, Hong Kong}

Background: Repeated resection via open approach is an effective treatment for post-operative recurrent hepatocellular carcinoma (HCC). However, there is still limited data on the application of laparoscopic approach to repeated resection for recurrent HCC in patients with prior liver resections. The aim of this study was to review our experience of laparoscopic re-resection in patients with postoperative tumor recurrence.

Materials and Methods: A total of 11 patients received laparoscopic re-resections for postoperative tumor recurrence in our center. Data was reviewed for demographics, primary and recurrent tumor characteristics, and perioperative outcomes.

Results: The median age of our patients was 63 years old (43-80 years old). All patients had Child A liver cirrhosis except two patients had normal livers and one patient had steatosis. Six patients had their first liver resection done by the open approach and the remaining five patients received the laparoscopic approach. The time to recurrence was 21 months (5-125 months). The recurrent tumor size was $20 \mathrm{~mm}$ $(12-50 \mathrm{~mm})$ and ten patients had solitary recurrence. Two patients had laparoscopic left lateral sectionectomy and the remaining nine patients had sub-segmentectomies. The perioperative blood loss was $100 \mathrm{ml}(10-600 \mathrm{ml})$ and the median operating time was 190 mins (131-352mins). The serum bilirubin and serum aspartate aminotransferase (AST) peaked on postoperative Day 1 in six (54.5\%) and seven patients (63.6\%) respectively. All patients showed normalizing trend of serum bilirubin level from postoperative Day 3 and of serum AST from postoperative Day 2 onwards. The postoperative morbidity rate was $18.2 \%$ and there was no hospital mortality. The median hospital stay was 7 days (5-23 days).

Conclusion: Our study showed that laparoscopic re-resection for recurrent HCC is feasible with satisfactory short-term outcomes, even in patients with previous major liver resections. Further studies are warranted to establish its role in the management of recurrent HCC.

\section{PE-020 \\ Long-Term Outcome of Laparoscopic Versus Open Liver Resection for Hepatocellular Carcinoma: A Case-Control Study with Propensity Score Matching}

Hyeyoung Kim Kyung-Suk Suh, Kwang-Woong Lee, Nam-Joon Yi, Geun Hong, Suk-Won Suh, Tae Yoo, Min-Su Park, YoungRok Choi, Hae Won Lee

Department of Surgery, Seoul National University College of Medicine, Seoul, Korea

Purpose: Laparoscopic liver resection (LR) for hepatocellular carcinoma (HCC) is usually applied to superficial and left side small lesions. Therefore, well designed comparative studies about the results of LR vs. open liver resection (OR) for HCC are difficult and still uncommon. The aim of this study was to compare the perioperative and long-term oncologic outcomes of LR vs. OR for HCC between well-matched patient groups.

Methods: Between January 2000 and March 2012, 205 patients (43 with intent to treat with LR, 162 OR) underwent primary liver resection of less than three segments for HCC in our center. To select a comparison group, propensity score matching (PSM) was used at 1:1 ratio with covariates of baseline characteristics including tumor characteristics. Outcomes were compared between the matched groups.

Results: The two groups were well balanced by PSM and 29 patients were matched respectively. In LR, there were more non-anatomical resection ( 65.5 vs. $34.5 \%, p=0.012$ ), less postoperative ascites ( 0.0 vs. $17.2 \%, p=0.025$ ), shorter hospital stay ( $7.69 \pm 2.94$ vs. $13.38 \pm 7.37$ days, $p<0.001)$. Except these, there were no significant differences in perioperative and long-term outcomes. The 1-, 3-, and 5- year survivals were 100,100 , and $92.2 \%$ in LR, and 96.5, 92.2 and $87.7 \%$ in $\mathrm{OR}$, each $(p=0.267)$. The $1-, 3-$, and 5 -year diseasefree survivals were $81.7,61.7$, and $54.0 \%$ in LR, and 78.6, 60.9, and $40.1 \%$ in OR, respectively ( $p=0.929$ ).

Conclusions: The outcome of LR for HCC was technically feasible and safe in selected patients and LR showed similar perioperative and long-term oncologic outcomes when compared with OR matched with PSM. 


\section{PE-021 \\ The Survival Outcomes of Hepatic Resection Compared with Transcatheter Arterial Chemoembolization for Hepatocellular Carcinoma with Portal Vein Tumor Thrombus}

Jung Min Lee ${ }^{1}$, Byoung Kuk Jang ${ }^{1}$, Yu Jin Kang ${ }^{1}$, Sang Min Lee ${ }^{1}$, Yoo Jin Lee ${ }^{1}$, Eun Sung Choi ${ }^{1}$, Jae Hyuk Choi ${ }^{1}$, Woo Jin Chung ${ }^{1}$, Jae Seok Hwang ${ }^{1}$, Koo Jeong Kang ${ }^{2}$, Young Hwan Kim ${ }^{3}$

${ }^{1}$ Department of Internal Medicine, ${ }^{2}$ Department of Surgery, ${ }^{3}$ Department of Radiology, Kermyung University, Daegu, Korea

Background: The prognosis of hepatocellular carcinoma (HCC) with portal vein tumor thrombosis (PVTT) is very poor. However, the treatment of HCC with PVTT is still controversial. We analyzed the results of transcatheter arterial embolization (TACE) compared with hepatic resection for HCC with PVTT.

Methods: Between 2000 and 2009, HCC with portal vein tumor thrombosis patients who received hepatic resection or TACE were included. Of patients, who had the main portal vein tumor thrombosis or Child-Turcot-Pugh score C were excluded. Total 240patients of hepatic resection, 19 cases were satisfied the inclusion criteria. These patients were compared with 67 patients who treated with TACE as an initial treatment for HCC with PVTT. PVTT was classified to 2 types; involve segmental branch (type I), extending to involve the right/left portal vein (type II).

Results: The median survival for the hepatic resection and TACE groups were 19.3 month and 6.4 month $(p<0.01)$. 1 -, 2- and 3-year overall survivals for the hepatic resection group and TACE group were $63.2 \%, 36.8 \%$ and $26.3 \%$ and $31.3 \%, 11.8 \%$ and $6.7 \%(p<0.01)$. On subgroup analyses, type I PVTT, tumor size $>5 \mathrm{~cm}$ were showed better results of the overall survival for the hepatic resection group than the TACE group ( $p<0.01, p=0.012)$.

Conclusions: Hepatic resection can help for patients with HCC with involving the segmental branch of PVTT, compared with TACE.

\section{PE-022 \\ Laparoscopic Liver Resection for Hepatocellular Carcinoma in Single Center Experience}

Ho Joong Choi and Il Young Park

Department of Surgery, Bucheon St. Mary's Hospital, College of Medicine, The Catholic University of Korea, Bucheon, Korea

Background: For the last two decades, minimally invasive surgery such as laparoscopic surgeries have competed with conventional open surgical procedures to establish themselves as the standard of care and in some cases have even done so. Also, in the area of liver surgery, laparoscopic liver resection has been increasingly used over the past two decades. Hereby, we report our experience about laparoscopic liver resection.

Methods: Between June 2006 and April 2013, 16 patients underwent laparoscopic liver resection for hepatocellular carcinoma (HCC) at Bucheon St. Mary's hospital. Of these 16 cases, 10 were laparoscopic partial hepatectomies, 5 were laparoscopic left lateral sectionectomies (LLS) and 1 was LLS with partial hepatectomy. We retrospectively reviewed the data of these patients. The median follow-up was 19 (1-82) months.

Results: Underlying liver disease was caused by HBV infection $(n=14)$ most commonly, followed by alcohol $(n=1)$ and other causes $(\mathrm{n}=1)$. All patients were associated with liver cirrhosis. Median operating time was 320 minutes (130-500 minutes) and blood loss was $400 \mathrm{ml}(150-800 \mathrm{ml})$. Two procedures were converted to open surgery due to bleeding and limitations by tumor location. There were no serious intraoperative or postoperative complications in this series. Also, there were no reoperation and postoperative mortality. All patients were shifted to the general ward within 2 days. Median postoperative hospital stay was 7 days $(5-27$ days). We experienced 7 recurrences (43.8\%) and four patients died due to HCC recurrence during follow-up period. Primary recurrence site was remnant liver in all recurred patients.

Conclusion: Our data showed that laparoscopic liver resection seems to be a feasible approach and can be safely performed in selected cases of hepatocellular carcinoma.

\section{PE-023 \\ Preoperative TACE Improves the Survival in the Resectable HCC with Highly Elevated AFP}

Do Seon Song ${ }^{1,2}$, Sung Won Lee ${ }^{1,2}$, Hee Yeon Kim ${ }^{1,2}$, Myeong Jun Song ${ }^{1,2}$, Si Hyun Bae $e^{1,2}$, Jong Young Choi ${ }^{1,2}$, Seung Kew Yoon 1,2, U Im Chang 1,2, Jin Mo Yang 1,2

${ }^{1}$ The Catholic University Liver Research Center, ${ }^{2}$ Departments of Internal Medicine, College of Medicine, The Catholic University of Korea, Seoul, Korea

Background: To evaluate the effect of preoperative TACE for resectable hepatocellular carcinoma (HCC) with highly elevated alpha-fetoprotein (AFP) ( $\geq 200 \mathrm{ng} / \mathrm{mL}$ ).

Methods: From August 2004 to March 2012, 297 consecutive patients with HCC underwent liver resection at Seoul St. Mary Hospital and St. Vincent Hospital. Among these patients, 39 patients had elevated AFP at diagnosis ( $\geq 200 \mathrm{ng} / \mathrm{mL}$ ). We retrospectively compared recurrence rate and overall survival between patients who underwent preoperative TACE (TACE-LR group, $\mathrm{n}=19$ ) and those who did not (LR group, 
$\mathrm{n}=20$ ). The patients who received other treatments besides TACE and whose follow up duration is less than a year were excluded. Time to recurrence and overall survival were analyzed by Kaplan Meier method and log-rank test.

Results: The median age of the patients was 52 years (range, 16-67) and median follow-up duration was 25 months (range, 6-107). Most patients (87.1\%) were related to chronic hepatitis B, and 26 patients $(66.7 \%)$ were male. All the patients were Child-Pugh class A. Three patients had portal vein invasion. Baseline AFP level was median $739.7 \mathrm{ng} / \mathrm{mL}$ (range, 231.68-11781.4). Tumor size at diagnosis was median $4 \mathrm{~cm}$ (range, 1.3-17). The recurrence rates were $63.2 \%$ for TACE-LR group and $50 \%$ for LR group $(P=0.523)$. Mean time to recurrence were $585.9 \pm 238.4$ days for TACE-LR group and $265.5 \pm$ 94.7 days for LR group $(P=0.206)$. The TACE-LR group had longer mean survival time than LR group (101.6 \pm 5.1 months vs. $80.9 \pm 9.3$ months, $P=0.031$ ).

Conclusions: Preoperative TACE improves the survival in the resectable HCC with highly elevated AFP ( $\geq 200 \mathrm{ng} / \mathrm{mL})$.

\section{PE-024 \\ Clinical Outcome of Surgical Intervention in Hepatocellular Carcinoma}

\begin{abstract}
Min Jin Kim ${ }^{7}$, Yun Nah Lee ${ }^{7}$, Young Seok Kim ${ }^{7}$, Youn Hee Cho', Jeong-Yeop Song', Sae Hwan Lee?, Sang Gyune Kim ${ }^{7}$, Jae Young Jang ${ }^{1}$, Hong Su Kim ${ }^{7}$, Boo Sung Kim', Jae Myung Lee ${ }^{2}$, Gyu Sung Choi ${ }^{3}$

Digestive Disease Center and Research Institute, ${ }^{1}$ Department of Internal Medicine, ${ }^{2}$ Department of Radiology, ${ }^{3}$ Department of General surgery, Soonchunhyang University School of Medicine, Bucheon, Korea
\end{abstract}

Background: Currently, treatment of hepatocellular carcinoma is diverse and is affected by numorous factors. Although, the mainstay of treatment is surgical resection in patients with adequate liver function. The aim of this study is to evaluate the clinical outcome of surgical resection in hepatocellular carcinoma patients of single center and to define the role of surgical resection.

Methods: From March 2002 to January 2013, a total of 163 patients with hepatocellular carcinoma treated with surgical resection at the Soonchunhyang university hospital, Bucheon, Korea were enrolled. We checked the patients' baseline characteristics and BCLC stage, Child-Pugh score, AFP, PIVKA II, laboratory findings. And we investigated the clinical outcome and prognostic factors of these patients. Survival and recurrence rates were evaluated by univariate and multivariate analysis using Cox's proportional hazards model.

Results: 31 patients were excluded because of reasons that expire on operation, $0 \& \mathrm{C}$, intraoperative RFA. Mean age of 132 patients (male 108, female 24) was 54.58 old (54-76 old). The mean follow up period was 28.5 month (1-120) and the etiology was HBV (108), HCV (8), alcohol (8), others (8). Chronic liver disease patients were 44 (33.3\%), liver cirrhosis patients were 88 (66.6\%). Child- pugh score was A (80), B (8). $\mathrm{C}(0)$ in liver cirrhosis patients. Treatment methods were only operation (87), operation after TACE (36), TACE after operation (4), operation with chemotherapy (4), operation after RFA (1). The mean size of tumor was $4.39 \mathrm{~cm}(0.8-18 \mathrm{~cm})$. The 2-, 5-, 10 year diseas-free survival after surgical resection were $84.6 \%, 62.6 \%, 47.2 \%$, respectively. The 2-, 5- and 10 -year overall survival after resection were $93 \%, 87 \%$ and $65 \%$. The disease free survival and overall survival according to treatment method were not significantly difference. Multivariated analysis revealed that total bilirubin and portal vein thrombosis were independent prognostic factors affecting disease-free survival (hazard ratio 1.283, 95\% CI 1.106 to $1.489, \mathrm{P}=0.001$ and hazard ratio $4.196,95 \%$ CI 1.706 to $10.322, \mathrm{P}=0.002$ ). Also portal vein thrombosis was the independent factor of poor overall survival (hazard ratio 4.434, $95 \%$ CI 1.151 to $17.077, \mathrm{P}=0.030$ ). In subanalysis, the 2 year overall survival between the group of only operation even though over the size $5 \mathrm{~cm}$ (14) and the group of operation after TACE downstaging (8) was not difference ( $86.7 \%$ vs $85.7 \%$ ). But the 2 year disease-free survival was difference between two groups (15.6\% vs $31.3 \%$ ).

Conclusions: Hepatectomy whether the combined with other treatment for hepatocellular carcinoma was a great treatment of choice. Portal vein thrombosis was significant prognostic factor of overall survival and disease free survival. Thus, in patients with portal vein thrombosis, special attention is required. In subgroup analysis, more cases of patient was needed to ensure the results.

\section{PE-025 \\ Clinicopathological Characteristics in Combined Hepatocellular- Cholangiocarcinoma: A Single Center Study}

Hee Chul Yu, Jae Do Yang, Sang In Bae, Hong Pil Hwang, Baik Hwan Cho

Department of Surgery, Chonbuk National University

Medical School and Hospital, Jeonju, Korea

Backgrounds: Combined hepatocellular carcinoma and cholangiocarcinoma (cHCC-CC) is an uncommon subtype of primary liver cancer that has rarely been reported in detail.

This study was done to evaluate the clinicopathological characteristics and prognostic factors of cHCC-CC.

Methods: The clinicopathological features of patients diagnosed and operated with cHCC-CC at Chonbuk National Hospital between January 1998 and December 2007 were retrospectively studied by comparing them with patients with hepatocellular carcinoma (HCC) alone who had undergone a hepatic resection during the same period. 
Results: Ten of the 152 patients who were undergone a hepatic resection were diagnosed with cHCC-CC and included in this study ( $\mathrm{M}: \mathrm{F}=8: 2$, median age: $51.9 \pm 11.1$ years). According to the parameters of the $7^{\text {th }}$ American Joint Committee on Cancer T staging, there were 76 (50.0\%), 44 (28.9\%), 9 (5.9\%), $18(11.8 \%)$ and 5 (3.3\%) patients with T stages I, II, IIIa, IIIb and IV cancer, respectively. Overall survival period was better in the HCC only group $(67.9 \pm 40.4$ months) than in the combined cHCC-CC group (22.7 \pm 40.1 months). 5 -year survival rate was $20 \%$ in the cHCC-CC group and $60 \%$ in the HCC group. The disease free survival for patients with cHCC-HCC and HCC were 16.3 and 50.9 months, respectively. The only significant clinicopathological factor identified by multivariate analysis was T staging. $(\mathrm{p}=0.05)$.

Conclusion: Even after curative hepatic resection, the presence of a cholangiocellular component appeared to be a poor prognostic indicator in patients with primary liver cancer.

T stage is the only independent significant prognostic factor associated with patient survival in our study.

\section{PE-026 \\ A Single Center Experiences of Laparoscopic Liver Resection for Hepatocellular Carcinoma \\ Ju Ik Moon, In Seok Choi \\ The Department of Surgery, Konyang University Hospital, Konyang University School of Medicine, Daejeon, Korea}

Aim: Try attempt of laparoscopic surgical treatment for hepatocellular carcinoma (HCC), according to the technical development of laparoscopic liver resection (LLR) has been increased. The aim of this study was to share our initial experiences with LLR for HCC of the new center.

Method: Between May 2006 and May 2013, a total of 28 LLR underwent in 27 patients with HCC at Department of Surgery, Konyang University Hospital, Daejeon, Korea.

Results: We underwent 16 wedge resections (57.1\%), 4 left lateral sectionectomies (14.3\%), 6 segmentectomies (21.4\%), 2 caudatectomies (7.1\%). Tumor size ranged from $0.7 \mathrm{~cm}$ to $5.0 \mathrm{~cm}$ (median $1.8 \mathrm{~cm}$ ) and the resection margin from 0.0 to $6.0 \mathrm{~cm}$ (median $0.95 \mathrm{~cm}$ ). Median operation time was $212.5 \mathrm{~min}$ (35-375min). The median hospital stay was 7 days (3-15days). Postoperative complication rates were $10.7 \%$. We experienced one mortality case. The median follow up duration was 26.0 months, and the 3-year disease free survival rate was $66.7 \%$. 5-year overall patient survival rate was $70.4 \%$.

Conclusion: Although our experiences are only a few, we think that the LLR for the treatment of HCC may be relatively safe in selected patients.

\section{PE-027 \\ Comparison of Transarterial \\ Chemoembolization and Hepatic Resection as the Initial Treatment for Large, Single, and Resectable Hepatocellular Carcinoma: A Propensity Score Analysis \\ Yun Bin Lee ${ }^{7}$, Dong Hyeon Lee ${ }^{7}$, Yuri Cho ${ }^{1}$, Su Jong Yu', Jeong-Hoon Lee', Hyo-Cheol Kim², Nam-Joon Yi ${ }^{3}$, Kwang-Woong Lee ${ }^{3}$, Jung-Hwan Yoon ${ }^{1}$, Jin Wook Chung', Kyung-Suk Suh ${ }^{3}$, Hyo-Suk Lee?, Chung Yong Kim ${ }^{7}$, Yoon Jun Kim ${ }^{1 *}$ \\ ${ }^{1}$ Department of Internal Medicine and Liver Research Institute, ${ }^{2}$ Department of Radiology, ${ }^{3}$ Department of Surgery, Seoul National University College of Medicine, Seoul, Korea}

Background/Aim: Hepatectomy is the standard treatment modality for single resectable HCC. However, transarterial chemoembolization (TACE) is broadly performed considering the technical difficulty of surgical resection, the postoperative hepatic decompensation, and the frequent recurrence after resection. This study was designed to compare the long-term survival of HCC patients who underwent hepatic resection or TACE as the initial treatment for large, single, and resectable HCC.

Methods: Between January 2003 to December 2007, 158 consecutive patients with large, single, and resectable HCC, 87 hepatic resection patients and 71 TACE patients were included. Propensity scores were generated to select matched patients. For the propensity model, 61 patients were selected from each arm of the study. Independent prognostic predictors were determined with the Cox proportional hazards model.

Results: The mean follow-up period was $65.5 \pm 33.65$ months.With similar baseline patient characteristics generated in the propensity score model, there was no significant difference in the long-term survival rates of the 2 groups of patients.The 1-, 3-, and 5-year overall survival rates for the initial hepatic resection group and the initial TACE group were $90.2 \%, 80.3 \%, 68.9 \%$ and $90.2 \%, 77.0 \%, 55.7 \%$, respectively $(P=0.231)$.In the Cox model, Child-Pugh score was the independent predictors of poor prognosis $(P=0.008)$.

Conclusions: In conclusion, hepatic resectionand TACE lead to comparable long-term survival rates for HCC patients with large, single, and resectable HCC. 


\section{PE-028 \\ Survival Outcome of Patients with Spontaneously Ruptured Hepatocellular Carcinoma Treated Surgically or by Transarterial Embolization}

Young-Joo Jin ${ }^{1}$, Jin-Woo Lee ${ }^{1 *}$, Hyun Jung Chung ${ }^{1}$, Young soo Kim ${ }^{1}$, Soon Gu Cho ${ }^{2}$, Yong Sun Jeon ${ }^{2}$

${ }^{1}$ Department of Internal Medicine, Inha University Hospital, Inha University School of Medicine, Incheon, ${ }^{2}$ Department of Radiology, Inha University Hospital, Inha University School of Medicine, Incheon, Korea

Background/Aims: Clinical outcomes of patients that underwent surgery, transarterial embolization (TAE), or supportive care for spontaneously ruptured hepatocellular carcinoma (HCC) were evaluated.

Methods: A consecutive 54 patients who diagnosed as spontaneously ruptured HCC at our institution between 2003 and 2012 were retrospectively enrolled. We compared clinical outcomes after surgical resection, TACE/TAE, or supportive care in these patients.

Results: The median age was 54 years (range, 30-87 years). The most common cause of HCC was hepatitis B virus infection (66.7\%). Median tumor size was $8.5 \mathrm{~cm}$ (range, 0.9-25.5 cm), and $4(7.4 \%)$ had HCCs within Milan criteria. Of the 54 patients, $6(11.1 \%)$ underwent surgery, $25(46.3 \%)$ TAE, and 23 (42.6\%) supportive care. The 2-, 4-, and 6-months cumulative survival rates at 2-, 4-, and 6- months were significantly higher in the surgery $(60 \%, 60 \%$, and $60 \%)$ or TAE (36\%, 20\%, and $20 \%)$ groups than in the supportive care group $(8.7 \%, 0 \%$, and $0 \%)$, respectively (each, $\mathrm{P}<0.01$ ), and tended to be higher in the surgical group than in the TAE group. Multivariate analysis showed that serum bilirubin (Hazard ratio [HR] 1.09, $\mathrm{P}<0.01$ ), creatinine (HR1.46, $\mathrm{P}=$ 0.04 ), and vasopressor requirement (HR 2.37, $\mathrm{P}=0.02$ ) were significantly associated with post-treatment mortality, whereas surgery (HR 0.41, P $<0.01$ ), and TAE (HR 0.13, P = 0.01 ) were inversely associated with post-treatment mortality.

Conclusions: Post-treatment survival after surgery or TAE was found to be better than after supportive care, and surgery tended to provide better survival benefit than TAE. However, serum bilirubin, creatinine level, and hemodynamic status should be considered during treatment decision making in patients with spontaneous HCC rupture.

\section{PE-029 \\ Transarterial Radioembolization Using Yttrium-90 Microspheres for Advanced Hepatocellular Carcinoma Prior to Living Donor Liver Transplantation}

Dong-Sik Kim ${ }^{1}$, Young-Dong $Y u^{1}$, Sung-Won Jung ${ }^{1}$, Nam-Hee Won ${ }^{2}$, Yun-Hwan Kim ${ }^{3}$, Sung-Ock Suh ${ }^{7}$

${ }^{1}$ Department of Surgery, Division of HBP surgery and Liver transplantation, Korea University Medical Center, Seoul, ${ }^{2}$ Department of Pathology, Korea University Medical Center, Seoul, ${ }^{3}$ Department of Radiology, Korea University Medical Center, Seoul, Korea

Use of Yttrium-90 has been previously reported as a potential modality of bridging or down staging for advanced hepatocellular carcinoma (HCC) mainly in the setting of deceased-donor liver transplantation. At the same time, living donor liver transplantation (LDLT) for HCC has been challenged by some researchers because of its fast-tracking to surgery and subsequent possibility of poor outcome. We reviewed medical records of 4 patients, who underwent transarterial radioembolization (TARE) using yttrium-90 labeled microspheres in an intent to downstage and proceeded to LDLT in our institution. All patients had hepatitis B-related liver cirrhosis and HCC exceeding UCSF criteria. Mean age was 58 7. Mean of maximum tumor size was $6.4 \pm 1 \mathrm{~cm}$ with mean of $4 \pm 0.8$ nodules. Three patients underwent 1 session of TARE and 1 patient underwent two sessions with mean dose of $1.2 \pm 0.5 \mathrm{GBq} /$ session. Three out of 4 patients showed favorable response. Both tumor size and tumor marker were markedly decreased. Three patients with favorable response underwent LDLT successfully after 13, 32, and 40 weeks after TARE. Pathologic examination of treated lesions showed complete tumor necrosis, but 2 patients showed viable well differentiated tumors of less than $2 \mathrm{~cm}$ size, which seems to be developed after TARE. All patietns currently alive without recurrence 15, 14, 11 months after surgery. One patient showed poor response and expired about 2 months after TARE without LDLT. Although long term follow up is needed, radioembolization using yttrium-90 showed promising results in terms of tumor downstaging and bridging to liver transplantation in addition to selecting patients with favorable tumor biology in patients with advanced HCC exceeding the UCSF criteria. 


\section{PE-030 \\ Peri-Transplant AFP Levels: Useful Markers Predicting Recurrence of Hepatocellular Carcinoma Patients who Uunderwent Liver Ttransplantation}

\author{
Kwang-Woong Lee, Nam-Joon Yi, YoungRok Choi, \\ Sukwon Suh, Jeong Moo Lee, Benjamin Agero, \\ Angelica Susan Cho, Yong Sun Jeon \\ Seoul National University Hospital, Seoul, Korea
}

\begin{abstract}
Aim: Alphafetoprotein (AFP) is one of useful tumor marker for predict tumor recurrence for hepatocellular carcinoma (HCC). However, little is known about the serial changes of AFP level from liver transplantation to recurrence and the relationships between AFP levels and prognoses. So, this study was conducted to investigate the clinical significance of the peri-transplant AFP levels for predicting recurrence in HCC patients who underwent liver transplantation.
\end{abstract}

Methods: We retrospectively reviewed the data from 231 HCC patients who underwent liver transplantation between February 2000 and December 2010. Peri-transplant AFP levels (pre-transplant level, 1 \& 3 months post-transplant level and level at recurrence) were analyzed for patient distribution and recurrence free survival.

Results: Before liver transplantation, there were 106 patients who had normal AFP levels and 125 who had high levels. Of 125 patients with high pre-transplant AFP levels, 97 had normal AFP levels 1 months after transplantation (Rapid normalized group) and 28 had persistently high levels (Nonrapid normalized group). Of rapid normalized group, 17 showed recurrence, of whom 11 had high AFP levels and 6 had normal levels. However, non-rapid normalized group, 24 showed recurrence, whom all had high AFP levels after recurrence. In subgroup analysis, if the patients with very high pretransplant AFP ( $>200 \mathrm{ng} / \mathrm{ml}$ ) had normalized post-transplant AFP, these patients had good recurrence free survival (RFS) as patients with normalized post-transplant AFP level (Mean RFS $=99$ vs. 115 months, $\mathrm{p}=0.24)$. But, if patients with not so high pre-transplant level $(20<$ (p normalized and 0.001$), \mathrm{mL}$ ng 20 Pre-transplant months, 25 vs. ((Mean level elevated persistently outcomes poor had they ml) AFP $\leq 200 \mathrm{ng} />$.

Conclusions: Not only pre-transplant AFP but also especially post-transplant AFP may provide useful clinical information concerning recurrence in HCC patients who underwent liver transplantation.

\section{PE-031 \\ Optimal Suggestive Screening Protocol after Living Donor Liver Transplantation for Hepatocellular Carcinoma \\ Nam-Joon Yi \\ Seoul National University Hospital, Seoul, Korea}

The survival after liver transplantation (LT) for hepatocellular carcinoma (HCC) is mainly affected by HCC recurrence. As indication is expanding, especially in living donor liver transplantation (LDLT), HCC recurrence is also increased. The aim of this study was to suggest an optimal screening protocol in terms of screening interval and tools by analyzing pattern of recurrence. From February 1999 to October 2010, 284 patients were underwent LDLT. Among them, 205 cases were retrospectively reviewed. Recurrence appeared in 55 cases. A risk index was developed using the statistically significant factors from a multivariate Cox model: preoperative AFP $>400$, Edmonson grade 3 or 4 , tumor size $>7 \mathrm{~cm}$, tumor number at least 7 and more, minor tumor necrosis in TACE group and positive microvascular invasion. Four risk groups were developed based upon risk index scores that showed different recurrence risk and time. The 1 year cumulative recurrence rates of Group I, II, III and IV were 0, 11, 51 and 100\%. Mean time to recurrence of group I, II, III and IV were 29, 11, 10, 4 months. Recurrence sites according to groups are not statistically significant. Across the group, extra-hepatic recurrence was developed more than $40 \%$ and combined multi-organ recurrence rates was $20 \%$. We suggested an optimal screening protocol based on different screening interval and tools according to risk groups. Our optimal screening protocol by analyzing pattern of recurrence is a valuable tool and highly recommendable in the clinical practice to improve the long term prognosis in recurred HCC.

\section{PE-032 \\ Significance of Clusterin Expression in Patients with Hepatocellular Carcinoma Undergoing Hepatic Resection \\ Gar-Yang Chau, Yet-Pan Chau \\ Taipei Veterans General Hospital, Taipei, Taiwan}

Background: Surgical resection offers hepatocellular carcinoma (HCC) patients a chance for a cure, but carries a high tumor recurrence rate. Clusterin is a highly conserved glycoprotein, enhancing cell aggregation in vitro. Clusterin upregulated in cancers of the breast, ovary, colon, prostate, kidney and HCC. Overexpression of clusterin has been correlated with tumor metastasis. This study evaluates the significance of serum clusterin levels and protein expression in resected tissue specimen in HCC patients. 
Methods: The sera and HCC and nontumor tissues of 140 HCC patients undergoing hepatic resection were prospectively collected. Serum clusterin levels were determined by enzyme-linked immunosorbent assay. The clusterin protein expression in resected specimen were examined by immunohistochemistry. Median followup time was 39.1 months.

Results: Mean serum clusterin levels were $13.0 \pm 5.9 \mathrm{ng} /$ $\mathrm{mL}$ (range, $1.0 \sim 36.7 \mathrm{ng} / \mathrm{mL}$ ). Patients with high serum clusterin level (> $14.5 \mathrm{ng} / \mathrm{mL}$ ) had significantly lower frequency of family history of HCC, poorer liver function, and higher frequency of tumor being multiple, presence of vascular invasion than those with low clusterin level $(\leq 14.5 \mathrm{ng} / \mathrm{mL})$. For patients with tumor size $>5 \mathrm{~cm}$, patient with a high serum clusterin level had significantly worse postoperative survival when compared to patients with low serum clusterin level $(\mathrm{p}=0.018)$. Clusterin protein was overexpressed in HCC tissues in 76 patients (54.3\%) and in nontumor liver tissues in 53 patients $(37.9 \%)$. Patients with overexpression of clusterin in nontumor tissue have worse postoperative survival rates $(\mathrm{p}=0.003)$.

Conclusions: In HCC patients, high serum clusterin levels and overexpression of clusterin in nontumor tissue related with worse prognosis after hepatic resection. Clusterin can be a prognostic indicator for HCC patients after resection.

\section{PE-033 \\ Living Donor Liver Transplantation Showed higher Incidence of Hepatocellular Carcinoma Recurrence than Deceased Donor Liver Transplantation}

Jeong-Moo Lee, Suk-Won Suh, Tae You, Jose Benjamin Navarro Burgos, Angelica Garcia, Min-Su Park, YoungRok Choi, Hyeyoung Kim, Geun Hong, Nam-Joon Yi, Kyung-Suk Suh, Choon-Hyuck David Kwon, Jae-Won Joh, Suk-Koo Lee

Department of Surgery, Seoul National University College of Medicine, Seoul, Korea

Background: Living donor liver transplantation (LDLT) becomes an important tool in hepatocellular carcinoma (HCC) treatment. However, the oncologic outcome between LDLT and deceased donor liver transplantation (DDLT) for HCC is controversial. In this study, we aimed to compare the HCC recurrence rates after LDLT and DDLT.

Methods: 216 patients (166 LDLTs and 50 DDLTs) who underwent LT for HCC within UCSF criteria were retrospectively reviewed. LDLT patients were divided into two groups: small living donor graft [small LDG, graft recipient weight ratio (GRWR) <1.0, n=59] and non-small LDG (GRWR $\geq 1.0$, $n=107)$. Patients were further stratified into low and high risk settings by the number of risk factors for recurrence.

Results: Recurrence free survival of LDLT was lower than that of DDLT. (88.6\% and $80.7 \%$ vs. $96.0 \%$ and $94.0 \%$ at
1 and 5 years, $p=0.045)$. LDLT was one of the independent risk factors for recurrence. There was no significant difference between two groups with regards to most clinical and tumor characteristics, however LDLT had a higher proportion of positive microvascular invasion. Recurrence free survival of small LDG was lower than that of DDLT. (86.4\% and 77.8\% vs. $96.0 \%$ and $94.0 \%$ at 1 and 5 years, $\mathrm{p}=0.041$ ). In low risk setting ( $\leq 1$ risk factor), LDLT showed comparable outcome with DDLT. However, the risk of recurrence was higher in LDLT than DDLT in high risk patients.

Conclusion: In conclusion, LDLT has different clinical characteristics and outcomes from DDLT. This should be considered to select optimal strategy for HCC.

\section{PE-034 \\ Prognostic Factor for the Recurrence of Patients with Far Advanced Hepatocellular Carcinoma after Living Donor Liver Transplantation}

Geun Hong, Kwang-Woong Lee, Sukwon Suh, Tae Yoo, Hyeyoung Kim, Min-Su Park, Youngrok Choi, Nam-Joon Yi, Kyung-Suk Suh

Seoul National University Hospital, Seoul, Korea

Background: We sometimes perform living donor liver transplantation for patients with far advanced hepatocellular carcinoma. Recurrence of transplant recipients with far advanced HCC including macrovascular invasion or huge HCC or numerous HCC is common and macrovascular invasion is often considered as contraindication of liver transplantation. The purpose of this study is to evaluate the predictive factor for recurrence of patients with far advanced HCC after LDLT.

Methods: We included 22 transplant recipients with HCC larger than $10 \mathrm{~cm}$ or more than 10 numbers or with macrovascular invasion preoperatively from January 2003 to October 2010. We investigated the preoperative tumor status and serum alpha-fetoprotein level. We investigated the recurrence and survival rates after living donor liver transplantation and the results after the use of TACE or resection or radiation therapy or chemotherapy and the effect of the sirolimus use for patients with advanced HCC after LDLT.

Results: 1 year disease free survival (DFS) and 2 year DFS were $40.9 \%$ and $23.9 \%$, respectively. 1 year survival rates (SR) and 2 year SR were $71.6 \%$ and $59.7 \%$, respectively. 6 patients were without tumor recurrence and the median survival duration was 25.4(13-85) months and the median survival duration of 16 patients with tumor recurrence was 12.37(3-40) months. There were no statistical differences of DFS and SR according to macrovascular invasion (MVi) and 2 year SR were $68.2 \%$ (no MVi) and 50.9\% (MVi). There were no significant different outcome according to treatment modality. However, there was significant different 2 yr DFS according to 
serum alpha-fetoprotein level, 54.5\% (AFP $<200 \mathrm{ng} / \mathrm{mL}$ ) and $0.00 \%$ (AFP $\geq 200 \mathrm{ng} / \mathrm{mL}$ ) after LDLT ( $\mathrm{p}=0.023$ ).

Conclusions: The patients with far advanced HCC have usually poor prognosis. However, the patients even with far advanced HCC can have better outcome after living donor liver transplantation if we select the candidates carefully by better selection criteria such as alpha-fetoprotein. They may have another chance for tumor free survival or comorbidity free survival or even long term survival after LDLT.

\section{PE-035 \\ Early Experience of Living Donor Liver Transplantation after Concurrent Chemoradiation Therapy Combined with Multimodal Treatment for Locally Advanced Hepatocellular Carcinoma}

Dai Hoon Han, Dong Jin Joo, Hyung Soon Lee, Su Hyung Lee, A-Lan Lee, Man Ki Ju, Kyu Ha Huh, Myoung Soo Kim, Jin Sub Choi, Gi Hong Choi, Soon Il Kim

Yonsei University College of Medicine, Seoul, Korea

Background: Recently localized concurrent intra-arterial chemotherapy with external beam radiation therapy (CCRT) was proposed to treat the patients with locally advanced hepatocellular carcinoma (HCC). Neoadjuvant CCRT can be considered as one of the method for down-staging of hepatocellular carcinoma for liver transplantation. Thus, we evaluated our early experiences of living donor liver transplantation (LDLT) for the patients with neoadjuvant CCRT and multimodal treatment.

Patients and Methods: A total 8 recipients who underwent LDLT after CCRT between December 2011 and November 2012 in our center were evaluated retrospectively.

Results: All patients got CCRT, transarterial chemoembolization (TACE) and 2 of them took liver resection before liver transplantation. Six patients were down-staged within Milan criteria on preoperative radiologic examinations. Seven of eight patients' histopathologic examinations of explanted livers were within UCSF criteria. There were three cases of recurrence and no mortality during follow-up period. Median disease-free survival was 10.5 months (5 15months) and median overall survival after initial diagnosis of locally advanced HCC was 26 months (16 42 months).

Conclusion: LDLT might increase the survival of the patients with locally advanced HCC who respond to CCRT.

\section{PE-036 \\ Serial Radiologic Findings and Pathologic Correlation of Hepatocellular Carcinoma after Radioembolization Using Yttrium-90 Microspheres}

\author{
Jung Suk Oh, Ho Jong Chun, Byung Gil Choi, \\ Hae Giu Lee, Si Hyun Bae, Jong Young Choi, \\ Seung Kew Yoon \\ Seoul St. Mary's Hospital, Seoul, Korea
}

Purpose: To present the serial radiologic findings and pathologic correlation in HCC patients who underwent radioembolization with yttrium-90 microsphere prior to resection.

Material and Methods: Four patients who underwent hepatectomy after 90 Y radioembolization were studied. Serial imagings before and after treatment were evaluated using the CT and MRI. The explants were examined by pathology. The serial radiologic findings and pathologic correlation were analyzed.

Results: Two target lesions in two patients showed complete pathologic necrosis. In two patients, a viable tumor was detected in the serial follow up CT scan and showed hepatocellular carcinoma with partial necrosis and apoptosis, surrounding large target necrosis in the pathology. At the serial FU CT scan, the peripheral rim enhancement in the early FU (1 month) was seen and disappeared in the delayed FU (>3 month) without additional procedures. This temporary peripheral enhancement was correlated with inflammatory change in the pathology.

Conclusions: The hypodense lesion in the post-radioembolization imaging is well correlated with histologic necrosis. Rim enhancement is an imaging characteristic that correlated with histologic inflammatory change.

\section{PE-037 \\ Usefulness of Fusion Imaging for the Treatment Evaluation of Radiofrequency Ablation for Hepatocellular Carcinoma \\ Yuki Makino ${ }^{7}$, Yasuharu Imai ${ }^{1}$, Takumi Igura', Masatoshi Hori ${ }^{2}$, Kazuto Fukuda ${ }^{1}$, Yoshiyuki Sawai ${ }^{1}$, Sachiyo Kogita ${ }^{7}$, Keisuke Isotani' ${ }^{3}$, Manabu Takamura ${ }^{3}$, Norihiko Fujita ${ }^{3}$, Takamichi Murakami4 \\ ${ }^{1}$ Department of Gastroenterology, Ikeda Municipal Hospital, ${ }^{2}$ Department of Radiology, Osaka University Graduate School of Medicine, ${ }^{3}$ Department of Radiology, Ikeda Municipal Hospital, ${ }^{4}$ Department of Radiology, Kinki University School of Medicine, Osaka, Japan}

Background: The treatment effect of radiofrequency ablation (RFA) has been conventionally assessed by comparing pre- and post-RFA images, side-by-side. This study is aimed to 
verify the usefulness of fusion imaging of pre- and post-RFA CT and MR, which we call CT and MR fusion imaging for more accurate treatment evaluation.

Methods: Analysis 1; CT fusion imaging was conducted for 102 hepatocellular carcinomas (HCCs) regarded as complete ablation by side-by-side interpretation at the time of RFA. Minimal ablative margin (MAM) was categorized into 3 groups as follows; $<0 \mathrm{~mm}$ (protruding from ablation zone), 0 to $<5 \mathrm{~mm}$, and $\geq 5 \mathrm{~mm}$, and the relation with local tumor progression (LTP) was examined. Analysis 2; Both CT and MR fusion imaging, using dynamic CT and gadolinium ethoxybenzyl diethylenetriamine pentaacetic acid (Gd-EOB-DTPA)enhanced MRI, respectively, were conducted for other 92 HCCs and the judgment results were compared between CT and MR fusion imaging.

Results: In the analysis 1, 3 year cumulative LTP rate of each group was $41.6 \%, 12.2 \%$, and $0.0 \%$, respectively, and multivariate analysis revealed MAM of $<0 \mathrm{~mm}$ was the only significant factor associated with LTP (hazard ratio 7.15, $\mathrm{p}<$ $0.001)$. In the analysis 2 , treatment evaluation by both CT and MR fusion imaging was possible in $57(62.0 \%)$ HCCs and the overall agreement between them for the categorization of MAM was good ( $\kappa$ coefficient $0.676, p<0.01$ ). MR fusion imaging enabled treatment evaluation in significantly larger number of HCCs than CT fusion imaging (85 (92.4\%) vs 62 (67.4\%), $\mathrm{p}<0.05)$.

Conclusions: MAM on CT fusion imaging was strongly related with LTP. Substantially equivalent treatment evaluation was possible on MR fusion imaging, and it can be applied even for HCCs undetectable on CT, by virtue of the higher detection sensitivity of Gd-EOB-DTPA-enhanced MRI. Fusion imaging enables objective and accurate treatment evaluation of RFA.

\section{PE-038 \\ Usefulness of the Extracted-Overlay Function in Volume Navigation System for the Treatment Planning and Evaluation of Radiofrequency Ablation for Hepatocellular Carcinoma \\ Yuki Makino ${ }^{1}$, Yasuharu Imai ${ }^{1}$, Yoshiyuki Sawai ${ }^{1}$, Sachiyo Kogita ${ }^{7}$, Takumi Igura ${ }^{1}$, Kazuto Fukuda ${ }^{7}$, Masatoshi Hori ${ }^{2}$, Norihiko Fujita ${ }^{3}$, Takamichi Murakami ${ }^{4}$ \\ ${ }^{1}$ Department of Gastroenterology, Ikeda Municipal Hospital, ${ }^{2}$ Department of Radiology, Osaka University Graduate School of Medicine, ${ }^{3}$ Department of Radiology, Ikeda Municipal Hospital, ${ }^{4}$ Department of Radiology, Kinki University School of Medicine, Osaka, Japan}

Background: The overlay function installed in Volume Navigation (GE Healthcare) enables overlapping display of a reference on ultrasonography (US). However, background US often becomes too obscure for practical use, because of overlaying different imaging modalities. Subsequently, we developed a novel technique which we call the extractedoverlay function, in which only an extracted tumor with a visualized ablative safety margin of $5 \mathrm{~mm}$ is overlaid on US, and investigated its usefulness.

Methods: Forty-one hepatocellular carcinomas (HCCs) treated by radiofrequency ablation (RFA) with this function were included. In RFA, an electrode was inserted targeting the overlaid tumor and margin. After ablation, contrast-enhanced US (CEUS) was conducted for treatment evaluation, by comparing the perfusion defect with the overlaid tumor and margin, and an ablative margin was categorized into 3 groups; (I) $<0 \mathrm{~mm}$ (protruding from ablation zone), (II) 0 to $<5 \mathrm{~mm}$, and (III) $\geq 5 \mathrm{~mm}$. Additional ablation was conducted in principal for HCCs in group I. The final judgment results were compared between CEUS and fusion imaging of pre- and post-RFA CT or MRI.

Results: The number of HCCs in groups I, II, and III on CEUS was 2, 32, and 5, respectively, and the other 2 HCCs were located too deep to evaluate. Additional ablation was impossible for the 2 HCCs in group I, for technical reasons. On fusion imaging, the number of HCCs in groups I, II, and III was 6,31 , and 4, respectively, and the overall agreement with CEUS was moderate ( $\kappa$ coefficient 0.46 ). Among 37 HCCs judged as complete ablation (groups II or III) on CEUS, 34 HCCs (91.9\%) were actually judged likewise on fusion imaging.

Conclusions: The extracted-overlay function enables effective treatment planning, without obscuring background US. Moreover, by combining with CEUS, it enables noninvasive treatment evaluation at the bedside, just after RFA.

\section{PE-039 \\ Monocyte Fraction of White Blood Cell Reflects Risk of Hepatocellular Carcinoma Recurrence after Curative Ablation}

Hong Seok Choi, Jeong Han Kim, Yong Hwang, Jun Jae Kim, Mi Jin Hong, Soon Young Ko, Won Hyeok Choe, So Young Kwon

Department of Internal Medicine, Konkuk University School of Medicine, Seoul, Korea

Background: Hepatocellular carcinoma (HCC) has high risk of recurrence after curative therapy and inflammation process involves progression of HCC. We aimed to investigate inflammatory marker that can predict HCC recurrence after radiofrequency ablation (RFA) for curative intent.

Methods: We retrospectively reviewed the medical records of HCC patients treated with RFA at Konkuk University Medical Center from year 2005 to 2010 . The last follow up was August 2012. The patients were divided according to recurrence and baseline characteristics including white blood cell 
(WBC) count with fraction and C-reactive protein (CRP) compared.

Results: Total 62 patients were included for analysis. There were 26 cases of recurrence (41.9\%). Thirty nine patients $(62.9 \%)$ were male and the most common etiology was hepatitis B $(n=48,77.4 \%)$. When we compared recurrence group with non-recurrence group, Child-Pugh score $(6.1 \pm 1.4$ vs. $5.3 \pm 0.8, p=0.015)$, monocyte fraction of WBC $(8.1 \pm 2.4 \%$ vs. $6.7 \pm 2.7 \%, \mathrm{p}=0.041)$, prothrombin time (PT, INR; $1.3 \pm 0.2$ vs. $1.2 \pm 0.2, p=0.040$ ) were higher and albumin $(3.4 \pm 0.6 \mathrm{~g} / \mathrm{dL}$ vs. $3.8 \pm 0.5 \mathrm{~g} / \mathrm{dL}, \mathrm{p}=0.036)$ was lower. Other baseline characteristics including neutrophil fraction, lymphocyte fraction and CRP were not significantly different between groups. Area under receiver operating curve (AUROC) of monocyte fraction was 0.675 and if we choose cut-off level of monocyte fraction as $7.1 \%$, Kaplan-Meier analysis with log-rank test showed significant difference for recurrence ( $p=0.006)$.

Conclusions: High monocyte fraction reflects increased risk of HCC recurrence after RFA. These patients need careful observation during follow up period and underlying mechanism have to be investigated.

\section{PE-040 \\ Comparison of the Outcomes between Colorectal Liver Metastases and Hepatocellular Carcinoma Treated with Radiofrequency Ablation: A Case-Control Study \\ Chao-Hung Hung ${ }^{1}$, Ko-Chao Lee ${ }^{2}$, Jing-Houng Wang', Hong Hwa Chen ${ }^{2}$, Chien-Chang Lu², Wang-Hseng $\mathrm{Hu}^{2}$, Sheng-Nan $L u^{1}$ \\ ${ }^{1}$ Division of Hepatogastroenterology, Department of Internal Medicine, ${ }^{2}$ Department of Colorectal Surgery, Kaohsiung Chang Gung Memorial Hospital, Chang Gung University College of Medicine, Kaohsiung, Taiwan}

Background/Aim: We aimed to compare the clinical outcomes after radiofrequency ablation (RFA) therapy between patients with colorectal liver metastases (CRLM) and hepatocellular carcinoma (HCC).

Methods: From 2008 to 2012, 34 patients with three or fewer nodules of CRLM undergoing RFA were enrolled. During the same period, an additional 204 age- and sex-matched HCC patients with similar characteristics of tumors (sizes and numbers) and Child-Pugh A were recruited from the HCC database. Both recurrence-free survivals and actuarial survival were evaluated by the Kaplan-Meier method.

Results: Complete tumor ablation was achieved in of 32 (94\%) of CRLM and 180 (88\%) of HCC patients, respectively $(P>0.05)$. The local tumor progression-free survival was lower in CRLM patients than in HCC patients $(P=0.028)$. Other significant factors associated with local recurrence were multinodularity $(P=0.006)$ and larger tumor size $(>2 \mathrm{~cm})(P=$ 0.034). Multivariate Cox's regression analysis identified that CRLM was an independent factor associated with local progression-free survival. However, there was no significant difference in the new recurrence-free and overall survival between CRLM and HCC patients $(P=0.286$ and 0.146 , respectively).

Conclusions: Despite a comparable primary success rate after RFA, patients with CRLM had a higher local recurrence rate than those with HCC.

\section{PE-041 \\ A Case of Photodynamic Therapy in HCC Biliary Invasion \\ Ja Won Koo, Won Jin Ko, Jae Jun Shim, Byung Ho Kim, Seok Ho Dong \\ Department of Internal Medicine, Kyung Hee \\ University, Medical College, Seoul, Korea}

Background: The prognosis of patients with obstructive jaundice caused by hepatocellular carcinoma (HCC) is dismal, because effective biliary drainage is difficult due to frequent malfunction of the drainage tube caused by hemobilia and/or tumor emboli. Photodynamic therapy (PDT) improves biliary patency and prolongs survival in hilar cholangiocarcinoma. There are several reports that PDT is an effective method for the palliative treatment of un-resectable cholangiocarcinoma. PDT for un-resectable cholangiocarcinoma may increase survival and improve quality of life. However, as we are aware, there are small in number reports of PDT for HCC with bile duct invasion. We report a case that received photodynamic treatment (PDT) for patients with bile duct invasion of unresectable HCC growth.

Case Report: A 46-year-old man was admitted because of jaundice and right upper quadrant pain. Abdominal US revealed biliary mass and he referred to KMC for further evaluation. He was diagnosed with HCC with both IHD and CHD invasion in abdominal CT and biopsy. Alternative treatment strategy such as transcatheter arterial chemoembolization (TACE) and radiotherapy was planned because of inoperability. Metalic stent was inserted in both IHD by endoscopic retrograde cholangiopancreatography to treat obstructive jaundice. But, obstructive jaundice did not resolve due to tumor ingrowth. Percutaneous transhepatic biliary drainage (PTBD) was performed. After severe obstructive jaundice was resolved, PDT was performed with $(2 \mathrm{~cm}, 585 \mathrm{sec}, 360 \mathrm{~J}$, $800 \mathrm{~mW}$.) for palliative therapy. After PDT, the amount of PTBD drainage was reduced and obstructive jaundice did not occur inspite of PTBD catheter was clamped. He received radiotherapy and did not developed recurrent jaundice for 10 weeks. 
Conclusions: There are lack of evidence the effectiveness of PDT on HCC. But in this case, biliary patency was seemed to be improved after PDT. It can be a good alternative choice of palliative methods in inoperable HCC but further study is needed.

\section{PE-042 \\ The Efficacy of Combination Therapy with Transarterial Chemoembolization and Sorafenib in Patients with Advanced Hepatocellular Carcinoma

\author{
Ki Tae Suk', Dong Joon Kim ${ }^{1}$, Heung Cheol Kim ${ }^{2}$ \\ ${ }^{1}$ Department of Internal Medicine, Hallym University \\ College of Medicine, ${ }^{2}$ Department of Radiology, Hallym \\ University College of Medicine, Seoule, Korea
}

Background: Transarterial chemoembolization (TACE) and sorafenib have been approved as a standard therapy in intermediate stage and advanced hepatocellular carcinoma (HCC), respectively. The efficacy of TACE, sorafenib, and combination therapy with TACE and sorafenib in patients with advanced HCC were evaluated.

Methods: Between April 2006 and April 2013, a total of 129 patients with advanced HCC who were treated with TACE $(\mathrm{n}=75)$, sorafenib $(\mathrm{n}=23)$, and combination therapy with TACE and sorafenib $(n=31)$ were retrospectively enrolled. Patient characteristics, CT findings, TACE findings, and survivals were assessed. Kaplan-Meier survival curve and Cox regression analysis was used to investigate the survival times and risk factors for mortality.

Results: The mean age of patients was $57.6 \pm 10.6$ years. The mean overall survival time and 3,6, and 12 -month survival rates were $24.4 \pm 2.8$ months, $77 \%, 60 \%$, and $48 \%$, respectively. The mean survival times of TACE, sorafenib, and combination therapy were $16.3 \pm 2.3,18.7 \pm 4.3$, and $29.4 \pm 4.1$ months, respectively. The 3, 6, and 12-month survival rate of each groups were $66 \%, 45 \%$, and $36 \%$ (TACE therapy), 86\%, $67 \%$, and $50 \%$ (sorafenib therapy), and $89 \%, 81 \%$, and $71 \%$ (combination therapy) (TACE vs. combination therapy: $p=$ 0.006 , sorafenib vs. combination therapy: $p=0.047)$. In the Cox regression analysis, Child-Pugh class C $(p=0.007$, OR 1.213, CI 1.054-1.396) and combination therapy $(p=0.028$, OR 0.475 , CI $0.244-0.923$ ) were related with mortality.

Conclusions: The combination therapy with TACE and sorafenib showed survival benefit compared with TACE or sorafenib monotherapy in patients with advanced HCC. In addition, Child-Pugh score was another risk factor of mortality.

\section{PE-043 \\ Risk Factors for Recurrence after Radiofrequency Ablation of Small HBV-Related Hepatocellular Carcinoma \\ Won Sohn ${ }^{1}$, Yong-Han Paik ${ }^{1 *}$, Min Woo Lee ${ }^{2}$, Hyunchul Rhim², Hyo Keun Lim², Ju Yeon Cho', Geum-Youn Gwak', Moon Seok Choi ${ }^{7}$, Joon Hyeok Lee', Kwang Cheol Koh', Seung Woon Paik', Byung Chul Yoo ${ }^{7}$ \\ ${ }^{1}$ Department of Internal medicine and ${ }^{2}$ Department of Radiology, Samsung Medical Center, Sungkyunkwan University School of Medicine, Seoul, Korea}

Background: Radiofrequency ablation as a curative therapy for hepatocellular carcinoma (HCC) is widely used. The aim of this study was to investigate risk factors for recurrence of HBV-related HCC after radiofrequency ablation.

Methods: A total of 173 patients at Samsung Medical Center, Seoul, Korea underwent radiofrequency ablation from January 2008 to December 2010 for small of HBV-related HCC $(\leq 3 \mathrm{~cm}$ in diameter). We analyzed the risk factors for recurrence of HCC after radiofrequency ablation.

Results: The median follow-up duration was 27.0 months. A total of 92 patients (53\%) with HBV-related HCC were recurred after radiofrequency ablation. Cumulative recurrence-free rates after radiofrequency ablation at 1-, 3-, and 5 -years were $81.1 \%, 46.4 \%$, and $35.1 \%$, respectively. A univariate analysis showed that risk factors for recurrence were the multi-nodularity (hazard ratio (HR) 2.23, $p=0.003$ ), pre-procedure HBV DNA levels $\geq 2000 \mathrm{IU} / \mathrm{mL}$ (HR 1.60, $p=$ 0.025), the presence of hepatitis B envelope antigen ( $\mathrm{HBeAg}$ ) (HR 1.56, $p=0.037$ ), Barcelona Clinic Liver Cancer (BCLC) stage A (HR 1.56, $p=0.038$ ). The independent risk factors for recurrence by multivariate analysis were the multi-nodularity (HR 1.95, $p=0.022$ ), pre-procedure HBV DNA levels $\geq 2000$ $\mathrm{IU} / \mathrm{mL}$ (HR 1.55, $p=0.041$ ).

Conclusions: Multi-nodularity and HBV DNA levels were associated with the recurrence after radiofrequency ablation in small HBV-related HCC. 


\section{PE-044 \\ Biplane Fluoroscopy-Guided Liver Radiofrequency Ablation with Planning Using Coronal Reformatted Images in Hepatocellular Carcinoma: Preliminary Experiences}

Yoodong Won, Sulim Lee, Yeil Kim, Kitae Kim

Uijeongbu St. Mary's Hospital, The Catholic University of Korea, Seoul, Korea

Background: To evaluate the feasibility and safety of biplane fluoroscopy-guided liver radiofrequency ablation with planning using coronal reformatted images.

Methods: We retrospectively evaluated nine nodules on liver dynamic multi-deterctor computed tomographic and fluoroscopic images in eight patients who underwent fluoroscopy-guided, percutaneous radiofrequency ablation to treat hepatocellular carcinoma. Pre-ablation planning using coronal reformatted images to search for safe puncture site and ideal targeting tract was done prior to all of the fluoroscopy-guided percutaneous radiofrequency ablation.

Results: A safe puncture site in the lateral aspect of the upper abdomen was found to be in the right seventh intercostal space in one patient, the right eighth intercostal space in four, the right ninth intercostal space in two, and the right tenth intercostal space in one patient. An ideal and safe track between the target lesion and the puncture site was delineated in all patients. Fluoroscopy-guided targeting was done in all nodules with the range of minimal error less than $8 \mathrm{~mm}$ compared to that of the planned ideal track.

Conclusions: An accurate and meticulous delineation of a safe puncture site and an ideal targeting track planned using coronal reformatted images allows the performance safer and more effective radiofrequency ablation under biplane fluoroscopy guidance.

\section{PE-045 \\ Treatment Outcome of Transarterial Chemoembolization with BCLC Stage 0 and A Hepatocellular Carcinoma.}

Hee Yoon Jang ${ }^{1}$, Yun Nah Lee ${ }^{1}$, Young Seok Kim ${ }^{7}$, Youn Hee Cho ${ }^{7}$, Jeong-Yeop Song ${ }^{1}$, Sae Hwan Lee, Soung Won Jung ${ }^{1}$, Sang Gyune Kim ${ }^{1}$, Jae Young Jang ${ }^{1}$, Hong Soo Kim ${ }^{1}$, Boo Sung Kim', Jae Myung Lee? Gyu Sung Choi ${ }^{3}$, Gab Jin Cheon ${ }^{4}$, Young Don Kim ${ }^{4}$

Digestive Disease Center and Research Institute, ${ }^{1}$ Department of Internal Medicine, ${ }^{2}$ Department of Radiology, ${ }^{3}$ Department of General Surgery, Soonchunhyang University School of Medicine, Bucheon, ${ }^{4}$ Departments of Internal Medicine, University of Ulsan College of Medicine, Gangneung Asan Hospital, Gangneung, Korea

Background: BCLC staging classification is used as standard treatment guideline of HCC. But there are many cases that we cannot keep the standards because of the various causes. Recently, the outcome of TACE is improved, there are increasing cases of TACE with stage 0 or A HCC patients. The aim of this study was to evaluate the treatment outcome and prognostic factors about survival rate of TACE with BCLC stage 0 and A HCC.

Methods: From January 2007 to December 2009, 440 consecutive patients diagnosed with HCC in Soonchunhyang University Hospital, Bucheon. Of these patients, 196 patients were received TACE or hepatic resection and we excluded the patients who were received previous TACE, RFA, PEI, IA chemotherapy or radiotherapy. We analyzed total 127 patients in this study retrospectively.

Results: Median age of patients was 57 years (range 38-91). Median follow-up period was 20 months (range $0-71$ ). Etiology of HCC was HBV in 95, HCV in 17, alcohol in 9. 104 patients were received TACE, 23 patients were received hepatic resection (OP). BCLC stage 0 was 13 (TACE $=10, \mathrm{OP}=3)$, BCLC stage A was $58(\mathrm{TACE}=45, \mathrm{OP}=13)$. There was no significant difference in survival between TACE and hepatic resection of BCLC stage 0 and A. (3-year survival rate TACE vs. OP $62.0 \%$ vs. $79.1 \%, p=0.362$ ). In TACE received patients, mean survival was 52.1 vs. 32.5 months for BCLC stage 0 and A vs. BCLC stage B, and 3-year survival rate was $62.0 \%$ vs. $44.2 \%$ ( $<<0.001)$. In BCLC stage 0 and $A$, progression free survival rate was 29.9 vs. 46.2 months for TACE vs. OP ( $p=0.086$ ). During the survival period, in TACE received patients, mean number of TACE procedure was 1.98 (range, 1-6) and mean period until $2^{\text {nd }}$ TACE was performed was 5.3 months. Univariate analysis for BCLC stage 0 and A HCC showed that bilirubin $>2 \mathrm{mg} / \mathrm{dl}$ (8.042, p = 0.030), albumin <3.5 g/dl, $\mathrm{Na}<135 \mathrm{mmol} / \mathrm{l}$, increasing MELD score, increasing MELD-Na score, increasing Child-Pugh score, mass number $\geq 2$, increasing TACE procedure number were the prognostic factors $(\mathrm{p}<0.05)$. 
Multivariate analysis showed that overall independent determinants were bilirubin $>2 \mathrm{mg} / \mathrm{dl}, \mathrm{Na}<135 \mathrm{mmol} / \mathrm{l}$, mass number $\geq 2(\mathrm{p}<0.05)$.

Conclusions: Although it shows a comparable outcome with surgical resection and TACE, in BCLC stage 0 and A HCC patients, surgical resection is a better therapeutic option than TACE. It should be considered active surgical intervention in BCLC stage 0 and A HCC patients.

\section{PE-046 \\ Predictability of ${ }^{18} \mathrm{~F}$-Fluorodeoxyglucose Positron Emission Tomography for Tumor Recurrence and Survival after TACE in Early and Intermediate Hepatocellular Carcinoma}

Min Jin Kim ${ }^{7}$, Yun Nah Lee ${ }^{7}$, Young Seok Kim ${ }^{7}$, Youn Hee Cho ${ }^{1}$, Jeong-Yeop Song ${ }^{1}$, Sae Hwan Lee ${ }^{1}$, Soung Won Jeong ${ }^{1}$, Sang Gyune Kim ${ }^{1}$, Jae Young Jang ${ }^{1}$, Hong Su Kim ${ }^{1}$, Boo Sung Kim ${ }^{1}$, Won Hyung Lee ${ }^{2}$, Jung Mi Park', Jae Myung Lee ${ }^{3}$, Tae Hee Lee ${ }^{4}$, Gab Jin Cheon ${ }^{5}$, Young Don Kim ${ }^{5}$

Digestive Disease Center and Research Institute, ${ }^{1}$ Department of Internal Medicine, ${ }^{2}$ Department of Nuclear Medicine, ${ }^{3}$ Department of Radiology, ${ }^{4}$ Department of Pathology, Soonchunhyang University School of Medicine, Bucheon, ${ }^{5}$ Departments of Internal Medicine, University of Ulsan College of Medicine, Gangneung Asan Hospital, Gangneung, Korea

Background: ${ }^{18}$ F-Fluorodeoxyglucose Positron Emission Tomography (PET) has a number of limitations in the use of hepatocellular carcinoma, because of variable FDG uptake in HCC. Nonetheless PET has been used as an assessment of biological behavior of HCC, recently. This study investigated between FDG uptake and prognosis, survival rate in early and intermediate group of HCC treated with transarterial chemoembolization (TACE).

Methods: From September 2006 to January 2012, a total of 194 patients undergoing ${ }^{18}$ F-Fluorodeoxyglucose PET before TACE at the Soonchunhyang university hospital, Bucheon, Korea were enrolled. Among them, only one of 59 patients had received a liver biopsy. And 24 pateints was early and intermediate HCC. We checked the patients' baseline characteristics and pathologic findings, laboratory findings. The FDG uptake was calculated by tumor maximum standardized upake value (SUV), liver mean SUV, the ratio of maximal tumor SUV to mean liver SUV.

Results: Mean age of 24 patients (male 19, female 5) was 66.39 years old. The mean follow up period was 22.06 months and the etiology was HBV (17), HCV (2), alcohol (3), others (2). Chronic liver disease patients were 7 (29.2\%), liver cirrhosis patients were 17 (70.8\%). BCLC stage was early (9), intermediate (15) and Edmandson-Steiner grade was I (5), II (12), III (4), IV (3). The mean session of TACE was 3.56 (1-7) and the mean size of tumor was $6.63 \mathrm{~cm}(1.3-14 \mathrm{~cm})$. The mean values

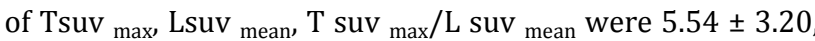
$2.00 \pm 0.33,2.75 \pm 1.90$, retrospectively. $\mathrm{T}$ suv $_{\text {max }} / \mathrm{L}_{\text {suv }}$ mean was the highest area under the receiver operating characteristic curve $(0.907, P=0.003)$ for predicting of recurrence among the calculated FDG uptake methods. The EdmandsonSteiner grade was not significantly associated with FDG uptake. Multivariated analysis revealed that tumor size, Tsuv max were the independent factors of overall survival (hazard ratio $1.043,95 \%$ CI 1.012 to $1.072, P=0.007$, hazard ratio $6.667,95 \%$ CI 1.480 to $30.031, P=0.013$ ).

Conclusions: FDG uptake calculated by Tsuv max was an effective prognostic factor of overall survival in early and intermediate HCC treated with TACE. And T suv ${ }_{\text {max }} / L_{\text {suv }}$ mean was the predictable method in tumor recurrance. Thus, 18 F-Fluorodeoxyglucose PET-CT might be considered a useful predictor of recurrence and survival in early and intermediate HCC treated with TACE.

\section{PE-047 \\ Palliative Treatment of Unresectable Hepatocellular Carcinoma with Obstructive Jaundice Using Biliary Drainage with Subsequent Transarterial Chemoembolization}

Sang Hyub Lee ${ }^{a}$, Jongkyoung Choi, ${ }^{a, b}$, Ji Kon Ryu ${ }^{a}$, Jin-Hyeok Hwang ${ }^{c}$, Dong-Won Ahn ${ }^{a}$,, Yong-Tae Kim ${ }^{a}$, Yong Bum Yoon ${ }^{a}$, Chang Jin Yoon ${ }^{e}$, Sung-Gwon Kang ${ }^{e}$, Jin Wook Chung ${ }^{f}$

aDepartment of Internal Medicine and Liver Research Institute, Seoul National University College of Medicine, Seoul National University Hospital, Seoul, bepartment of Internal Medicine, National Medical Center, Seoul, 'Department of Internal Medicine, Seoul National University Bundang Hospital, Seongnam-si, dDepartment of Internal Medicine, Seoul National University Boramae Medical Center, Seoul, eDepartment of Radiology, Seoul National University Bundang Hospital, Seongnam-si, fDepartment of Radiology, Seoul National University Hospital, Seoul, Korea

Background: Nonsurgical biliary drainage is considered as a priority for obstructive jaundice associated with unresectable hepatocellular carcinoma (HCC). Successful drainage allows the patient to receive anti-tumor therapy, such as transartieral cheoembolization (TACE). However, only limited data are available on clinical outcomes in patients who treated biliary drainage with subsequent TACE. This study evaluated the clinical outcome of biliary drainage with subsequent TACE in unresectable HCC patients with obstructive jaundice.

Methods: This retrospective study evaluated adult patients with unresectable HCC. A total of 60 patients who received endoscopic biliary drainage (ERBD) or percutaneous 
transhepatic biliary drainage (PTBD) in 2 tertiary-care referral centers. The primary outcome was successful biliary drainage overall survival.

Results: Successful drainage was achieved in 39 (65\%) of all patients. The median survival of 39 patients in whom successful drainage was achieved, regardless of which procedure was performed, was much longer than that of 21 patients without successful drainage (147 days vs. 38 days, respectively, $P<.001$ ). In particular, the median survival was longer in 17 patients who underwent TACE after achieving successful drainage than in 22 patients who were treated conservatively after achieving successful drainage (410 days vs. 77 days, respectively, $P<.001$ ). Multivariable analysis in 39 patients in the successful drainage group showed that TACE (hazard ratio, 0.15: 95\% confidence interval: 0.05-0.45, $P=.001]$ was an independent predictive factor of a favorable outcome.

Conclusions: Effective palliation by successful biliary drainage with subsequent TACE might prolong the survival in patients with obstructive jaundice caused by unresectable HCC.

\section{PE-048 \\ Transarterial Chemoembolization Using Doxorubicin-Eluting Beads for Treatment of a Single Small Hepatocellular Carcinoma: A Pilot Study}

\author{
Jin Dong Kim ${ }^{7}$, Young Nam Kim ${ }^{1}$, A Ra Choi', \\ Ban Seok Lee ${ }^{1}$, Byung Hyo Cha ${ }^{1}$, Sang Jin Lee ${ }^{1}$, \\ Se Woo Park ${ }^{1}$, Won Joong Jeon ${ }^{1}$, Ha Hun Song ${ }^{2}$, \\ Hyun Kim² \\ Departments of ${ }^{1}$ Internal Medicine and ${ }^{2}$ Radiology, \\ Cheju Halla General Hospital, Jeju, Korea
}

Background: Surgical resection, liver transplantation, and percutaneous ablation are generally accepted as amenable treatments for small hepatocellular carcinoma (HCC). Recently drug-eluting beads (DEB) which had the ability to sequester chemotherapeutic agent from solution and release it in a sustained and controlled mode were introduced for transarterial chemoembolization (TACE). The aim of this study was to evaluate feasibility and safety of DEB-TACE for the treatment of single small HCC.

Methods: We assessed retrospective data of 15 patients who had unresectable HCC or were unwilling to receive resection or percutaneous ablation therapy from October 2010 to October 2012. In all patients, diagnosis of a small $(<3$ centimeter in greatest dimension) solitary HCC was ascertained by gadoxetic acid enhanced MRI following dynamic CT. After a single session of DEB-TACE, treatment response was regularly followed up with CT or MRI. The primary end point was local tumor progression and second end point was treatment-related complication.
Results: Median age of the patients was 62 years (range: 43-79). After a median follow-up of 14.1 months (6.5-30.4), sustained complete response was observed in 11 patients $(73.3 \%)$. Of the 15 patients who underwent DEB-TACE, 2 patients had suboptimal result of embolization on first followup imaging (post 1-2 months). Local recurrence were noted in another 2 patients (9.1 and 10.4 months after DEB-TACE, respectively), so estimated overall cumulative local recurrence free intervals after a single DEB-TACE were $86.7 \%$ at 6 months and $68.9 \%$ at 12 months. Two patients (13.3\%) experienced gangrenous cholecystitis requiring cholecystectomy as treatment-related adverse event.

Conclusions: Considering previous studies of radiofrequency ablation, local recurrence and complication rates after DEB-TACE are relatively high. These data indicate that DEB-TACE should be applied selectively in patients with a single small HCC if other therapeutic modality is unfeasible.

\section{PE-049 \\ Feasibility and Safety of Radioembolization Via extrahepatic Arteries of Hepatocellular Carcinoma}

Gyoung Min Kim, Yun Hwan Kim, Sung Bum Cho, Hwan Hoon Chung, Tae-Seok Seo, Seung Hwa Lee

Department of Radiology, Anam Hospital, Korea

University Medical Center, Seoul, Korea

Purpose: To assess the feasibility and safety of radioembolization via extrahepatic collateral arteries of hepatocellular carcinoma (HCC).

Materials and Methods: From December 2008 to April 2013, 94 procedures of radioembolization was performed for 75 patients with HCC. Among them, Three patients who received yittrium-90 via extrahepatic arteries were included. All patients underwent diagnostic angiography and lung shunt scan, and the treatment plans were determined by interdepartment conference of interventional radiology and nuclear medicine. Tumor response was obtained using computed tomography and complication was evaluated. Child-Pugh classifications before and after treatment were also evaluated.

Results: The patients consisted of two males and one female. Mean age was 57.0 years. Two patients received yittrium-90 via right inferior phrenic artery, and one patient via right inferior adrenal artery. Right inferior phrenic artery was the only artery used in drug-delivery in one patient. Partial response was achieved in one patient, Stable disease in one patient, and progressive disease in one patient. There were no complications except tolerable abdominal pain. Child-Pugh classification was aggravated in one patient (A6 -> B8).

Conclusion: Though further study is needed, radioembolization via extrahepatic artery is possible without major complication. 


\section{PE-050 \\ Comparison of the Effectiveness between Doxorubicin-Eluting Beads and Conventional Transarterial Chemoembolization for Hepatocellular Carcinoma}

\author{
Eun Haeng Jeong ${ }^{1}$, Byung Ik Kim ${ }^{1}$, Hyun Pyo Hong ${ }^{2}$, \\ Yong Kyun Cho ${ }^{7}$, Hong Joo Kim ${ }^{1}$, Woo Kyu Jeon ${ }^{1}$ \\ ${ }^{1}$ Department of Internal Medicine, ${ }^{2}$ Department \\ of Radiology, Sungkyunkwan University Kangbuk \\ Samsung Hospital, Seoul, Korea
}

Background: Drug-eluting beads (DC-Bead ${ }^{\circledR}$ ) has been applied to enhance drug delivery to the tumor effectively. This study compared that the efficacy between transarterial chemoembolization (TACE) with DC-bead ${ }^{\circledR}$ loaded by doxorubicin and conventional TACE with lipiodol-based regimen for treating hepatocellular carcinoma (HCC).

Methods: From October 2010 to December 2011, 33 patients were treated by DC-Bead ${ }^{\circledR}$ TACE and 158 patients by conventional TACE. Patients with any other cancer, receiving other treatment modality (hepatectomy, radiofrequency ablation, radiation therapy), unmeasurable HCC (diffuse type), hepatic rupture and follow up loss were excluded. After exclusion criteria were applied, 21 patients in DC-Bead ${ }^{\circledR}$ TACE group and 30 patients in conventional TACE group were analyzed. The primary end points were treatment response at first follow up image study (average 51 days).

Results: There was no significant difference of the response by modified Response Evaluation Criteria in Solid Tumors (RECIST 1.1). The responses by RECIST 1.1 were complete response (CR) 28.6\%, partial response (PR) 4.8\%, stable disease (SD) 61.9\%, progressive disease (PD) $4.8 \%$ in DC-Bead ${ }^{\circledR}$ TACE group versus CR 33.3\%, PR 0\%, SD 43.3\%, PD $23.3 \%(p=0.137)$ in conventional TACE group. Both groups showed improvement in the modified UICC stage (DC-Bead ${ }^{\circledR}$ TACE $p=0.009$; conventional TACE, $p=0.001$ ) but not in the BCLC stage. There was no significant difference between two groups in improvement of modified UICC stage $(p=0.285)$ and liver toxicity $(\mathrm{p}=0.903)$.

Conclusions: There was no significant difference of the response between DC-Bead ${ }^{\circledR}$ TACE group and conventional TACE group. Therefore, the use of DC-Bead ${ }^{\circledR}$ TACE should be selected the appropriate patient. When TACE-response assessment was evaluated, the UICC stage was better than the BCLC stage in this study.

\section{PE-051 \\ The Usefulness of Contrast-Enhanced Ultrasonography in Early Detection of Hepatocellular Carcinoma Viability after Transarterial Chemoembolization - Preliminary Data}

Moon Young Kim ${ }^{1,2}$, Soon Koo Baik 1,2, Youn Zoo Cho Won Ki Hong', Hye Won Hwang ${ }^{1}$, Jin Hyung Lee', Myeong Hun Chae ${ }^{1}$, Seung Yong Shin ${ }^{1}$, Jung Min Kim ${ }^{1}$, Sang Ok Kwon ${ }^{1}$

${ }^{1}$ Department of Internal Medicine, ${ }^{2}$ Department of Cell Therapy and Tissue Engineering, Wonju Severance Christian Hospital, Yonsei University Wonju College of Medicine, Wonju, Korea

Background: Transarterial chemoembolization (TACE) is one of the most commonly applied therapeutic method for hepatocellular carcinoma (HCC). The therapeutic effect after TACE is usually assessed by dynamic liver computer tomography (CT) scan at several weeks later from TACE. In general, compact dense deposition of lipiodol is accepted as success sign of TACE. However, dense deposition of lipiodol also could mask the viable HCC tissue enhancement in the CT scan. The size of $2^{\text {nd }}$ generation microbubble ultrasonography contrast agent (UCA) is smaller than red blood cell as about $2.4 \mu \mathrm{m}$ so, the contrast-enhanced ultrasonography (CEUS) using $2^{\text {nd }}$ generation microbubble UCA could be effective in detection of small part of viability and patency of vessel in HCC after TACE without interference in assessment by lipiodol. So, in this preliminary study, we investigated whether the arterial enhancement in CEUS at 4week after TACE can predict or early detect HCC viability compared to CT scan.

Methods: Totally, 12 patients were enrolled in this study. They all received CEUS, CT scan and MRI at baseline and 4week, 12week after TACE. The primary end-point was HCC viable tissue detection in Gd-EOM-DTPA-enhanced magnetic resonance imaging (MRI) after 4weeks or 12 weeks later. Arterial phase enhancement was defined as positive finding for remained viable HCC in CEUS or CT scan. The independent variable was the positivity of 4 week CEUS.

Results: Among 12 patients, 8 patients showed positive finding for primary end-point (MRI positive at 4 week or 12week). At 4week, CEUS, CT and MRI showed positive findings in 8 (66.7\%), 3 (25\%) and 4 (33.3\%) patients respectively. All Patients who had 4 week CEUS positive finding ( $\mathrm{n}=$ 8) showed MRI positive and remained viable HCC at 4week or 12 week $(\mathrm{p}=0.002)$. Among these 8 patients, 5 patients presented 4week CT scan negative. These 5 patients showed all remained HCC viable tissue, 1 patient showed 4week MRI positive finding and the other 4 patients showed 12 week MRI positive. On the contrary, among patient who showed 4week CT scan positive without 4week CEUS positive, no one finally diagnosed as having viable HCC positive. Especially, among 8 patients of 4 week CEUS positive, 4 patients (50\%) did not 
presented 4week MRI positive and they all finally confirmed to have remained HCC tissue just by 12 week MRI test.

Conclusions: In assessment of therapeutic response of TACE, early 4week CEUS showed excellent result in diagnosis and prediction of remained viable HCC. However, this result was derived from just small samples as preliminary study and has to be followed by more advanced well designed large population study.

\section{PE-052 \\ Staging and Classification Systems as Prognostic Factors of Advanced Hepatocellular Carcinoma after Hepatic Arterial Infusion Chemotherapy}

\author{
Myung Jin Oh, Heon Ju Lee, Chan Seo Park
}

Department of Internal Medicine, Yeungnam University College of Medicine, Daegu, Korea

Background: As an alternative for intractable advanced hepatocellular carcinoma (HCC), hepatic arterial infusion chemotherapy (HAIC) with 5-fluorouracil (5-FU) and cisplatin may have survival benefits. We aimed to determine survivalrelated staging and classification systems for advanced HCC after HAIC.

Methods: From January 2009 to December 2011, a total of 54 patients who received only HAIC with $5-\mathrm{FU}(750 \mathrm{mg} / \mathrm{m} 2$ on days $1-4)$ and cisplatin (25 mg/m2 on days $1-4)$ as palliative therapy for advanced HCC were selected. We retrospectively analyzed the relationship between overall survival (OS), progression-free survival (PFS) and staging or classification systems such as MELD score, Child-Pugh class, BCLC stage, Okuda stage, modified UICC stage, and CLIP stage.

Results: Median age of selected patients was 59.0 years. The causes of HCC were hepatitis B virus (70.4\%), hepatitis C virus (3.7\%), and unknown (26.0\%), respectively. Mean cycle of HAIC was $4.0 \pm 2.9$ times. Median OS of enrolled patients was 5.0 months, and was significantly different in the classification according to Child-Pugh class, BCLC stage, Okuda stage, and CLIP stage, respectively ( $\mathrm{P}<0.05)$. In addition, median PFS of enrolled patients was 4.0 months, and was statistically significant in the classification according to BCLC stage and CLIP stage, respectively $(\mathrm{P}<0.05)$. However, median OS and PFS were not significantly different in modified UICC stage and MELD score, respectively ( $\mathrm{P}>0.05$ ). Exclusively, Okuda stage and CLIP stage were independent prognostic staging systems of OS and PFS of advanced HCC after HAIC, respectively (HR: 3.175, 95\% CI: 1.152-8.752, P = 0.026; HR: 55.809, 95\% CI: 3.450-902.721, P = 0.005).

Conclusions: We demonstrated that Okuda stage and CLIP stage might be survival prognostic parameters of HAIC for advanced HCC.

\section{PE-053 \\ Real-life Data of the Use of Sorafenib in Hepatocellular Carcinoma (HCC) Patients: A Single Centered Study}

Eileen L. Yoon, Jong Eun Yeon*, Ji Hye Je, Yang Jae Yoo, Keun-Hee Kang, Hyun Jung Lee, Sang Jun Suh, Ji Hoon Kim, Yeon Seok Seo, Hyun Joon Yim, Kwan Soo Byun

Division of Gastroenterology and Hepatology, Department of Internal Medicine, Korea University College of Medicine, Seoul, Korea

Background: Sorafenib is an only treatment proven to be beneficial for survival in advanced HCC patients. However, aggravation of the patients' performance status (PS) frequently limits the use of sorafenib in field-practice. We aimed to assess the real-world efficacy of sorafenib and factors significantly resulting in the early discontinuation of sorafenib use less than 4-weeks.

Methods: We retrospectively analyzed the medical records of 102 patients who were treated for advanced HCC with sorafenib in Korea University Guro Hospital, between 2008 and 2012. Patients were divided into "Early discontinuation (ED) group (n=32)" and "Traceable for response (TR) group ( $\mathrm{n}=70)$ ) according to the treatment duration.

Results: Best response rates of our center in the TR group were as follow: CR $(3 / 70,4.3 \%)$, PR (9/70, 12.9\%), SD (26/70, $37.1 \%)$, and PD $(32 / 70,45.7 \%)$. The median overall survival and time to progression were 11.29 months and 2.93 months. Cox regression analysis revealed that worse PS upon discontinuation in the TR group and lymph node involvement were negatively related to the survival [HR 2.92 (95\% C.I 1.37-6.25, $\mathrm{p}=0.005$ ) and HR 3.85 (95\% C.I 1.33-11.16, p = 0.013), respectively].

Baseline characteristics of patients, liver function, and tumor characteristics were compared between the groups. ECOG PS 0 patients accounted for $31.3 \%$ and $70.0 \%$ in ED and TR group ( $\mathrm{p}<0.001)$. Prevalence of Child A patients were $50.0 \%$ and $82.9 \%$ in the ED and TR group, respectively $(\mathrm{p}=$ 0.002 ). Infiltrative type of tumor and presence of macrovascular invasion were more frequent in the ED group $(p=0.014$ and $\mathrm{p}=0.016$ ). Among the reasons of treatment discontinuation, aggravation of PS was noted as high as $65.6 \%$ and $37.1 \%$ in the ED and TR group, respectively ( $p=0.010$ ).

Conclusions: Not only tumor characteristics, but also the baseline PS and aggravation of PS significantly affected the field-practice of sorafenib in both the ED and TR group. 


\section{PE-054 \\ Lamivudine and Adefovir Combination Therapy for Lamivudine Resistance in Hepatitis B Related Hepatocellular Carcinoma Patients}

Jeong Han Kim, Hong Seok Choi, Yong Hwang, Jun Jae Kim, Soon Young Ko, Won Hyeok Choe, So Young Kwon

Department of Internal Medicine, Konkuk University School of Medicine, Seoul, Korea

Background: Lamivudine (LAM) and Adefovir (ADV) combination therapy has been accepted as one of the best treatments for LAM resistant chronic hepatitis B (CHB) patients. The aim of this study was to investigate the efficacy of combination therapy in hepatocellular carcinoma (HCC) patients.

Methods: We reviewed the medical records of $\mathrm{CHB}$ patients who developed LAM-resistance and were treated with LAM and ADV combination therapy for more than 6 months. We assessed the virological response (VR, undetectable HBV DNA), biochemical response (BR, ALT normalization) and compared HCC patients and non-HCC patients.

Results: A total of 104 patients $(\mathrm{HCC}=19$, non-HCC $=85$ ) were analyzed. VR rates were not significantly different between the HCC and the non-HCC groups: $33.3 \%$ vs. $55.6 \%$ at 12 months ( $\mathrm{p}=0.119$ ), $58.3 \%$ vs. $67.2 \%$ at 24 months ( $\mathrm{p}=$ 0.742 ), $50 \%$ vs. $69.8 \%$ at 36 months ( $p=0.280$ ), $66.7 \%$ vs. $71.0 \%$ at 48 months $(\mathrm{p}=1.000)$. BR rates were also not significantly different: $55.6 \%$ vs. $84.0 \%$ at 12 months ( $\mathrm{p}=0.021)$, $58.3 \%$ vs. $83.8 \%$ at 24 months ( $p=0.057$ ), $70.0 \%$ vs. $77.8 \%$ at 36 months ( $\mathrm{p}=0.687), 66.7 \%$ vs. $80.6 \%$ at 48 months $(\mathrm{p}=$ 0.591).

Conclusions: The efficacy of LAM-ADV combination therapy is comparable in HCC patients as well as non-HCC patients.

\section{PE-055 \\ Is Antiviral Therapy Necessary to Hepatitis B Related Hepatocellular Carcinoma Patients Treated with Sorafenib?}

Yong Hwang, Jeong Han Kim, Hong Seok Choi, Jun Jae Kim, Soon Young Ko, Won Hyeok Choe, So Young Kwon

Department of Internal Medicine, Konkuk University School of Medicine, Seoul, Korea

Background: Nucleos(t)ide analogue (NA) can protect disease progression in chronic hepatitis B (CHB) patients. For hepatocellular carcinoma (HCC) patients, NA helps to make lower recurrence rate after curative treatment. On the other hands, advanced stage HCC patients have poor life expectancy about several months. Whether antiviral therapy using NA can help such patients is still unknown. We aimed to investigate usefulness of antiviral therapy for advanced stage HCC patients treated with sorafenib.

Methods: Medical records of HCC patients treated with sorafenib were analyzed retrospectively. The patients were divided two groups. Group A was non-antiviral therapy group, such as HCC patients caused by other than hepatitis B (hepatitis C, Alcohol...etc) and hepatitis B related HCC patients without antiviral therapy. Group B was antiviral therapy group treated with NA. Progression free survival (PS) and Overall survival (OS) were compared between these two groups.

Results: Of total 60 patients, Group A was 17 and Group $B$ was 43 . Male was predominant in both groups $(14,82.4 \%$ vs. $36,81.8 \%, \mathrm{p}=1.000)$. Child-Pugh class A/B/C were 6 $(35.3 \%) / 10(58.8 \%) / 1$ (5.9\%) in Group A and 22 (50.0\%)/19 $(43.2 \%) / 2(4.5 \%)$ in Group B ( $p=0.650)$. PS was $74.5 \pm 95.8$ days in Group A and 74.0 \pm 84.1 days in Group B ( $p=0.984)$. Mean OS of Group A was 76.0 \pm 88.5 days and that of Group B was $133.3 \pm 307.3$ days $(\mathrm{p}=0.455)$.

Conclusions: PS and OS were not significant different between two groups. In consideration of cost effectiveness, antiviral therapy may be not mandatory.

\section{PE-056 \\ Liver Abscess in Advanced Hepatocellular Carcinoma after Sorafenib Treatment}

Seung Kak Shin, Hae Lim Baek*, Young Kul Jung, Yun Soo Kim, Oh Sang Kwon, Ju Hyun Kim,

Duck Joo Choi, Soo Young Park, Min Young Rim, Hyeonsu Park, In Ku Yo, Seung Jun Jang, Hyunhwa Yoon

Internal Medicine, Gachon University Gil Medical Center, Incheon, Republic of Korea, Incheon, Korea

Hepatocellular carcinoma (HCC) represents a critical issue in global health and is listed as the third most common cause of cancer-related deaths worldwide. The majority of patients who present with HCC are already at an advanced stage, and the tumors are unresectable. Sorafenib is a multikinase inhibitor of the vascular endothelial growth factor (VEGF) pathway recently introduced in the therapy of advanced HCC. Recent studies have shown the beneficial effects on survival time of the oral multikinase inhibitor, sorafenib. However, many patients experience diverse side effects, some of them are severe and unexpected. To date, liver abscess has not been documented in association with a HCC patient treated with sorafenib. Here, we report a case with liver abscess, as adverse effect of the therapy with sorafenib. 


\section{PE-057 \\ The Role of Viral Load of Hepatitis B in the Recurrence of Early Stage Hepatocellular Carcinoma after Complete Ablation of Radiofrequency Ablation}

\author{
Kwong-Ming Kee, Kuo-Ching Chang, Jing-Houng Wang \\ Yuan-Hung Kuo, Sheng-Nan Lu \\ Division of Hepatogastroenterology, Department of \\ Internal Medicine Kaohsiung Chang Gung Memorial \\ Hospital and Chang Gung University College of \\ Medicine, Taoyuan, Taiwan
}

Background: Recurrence of hepatocellular carcinoma (HCC) is a major issue in early stage of disease. Aims: To investigate the role of viral load of hepatitis B virus (HBV) in the recurrence of early stage HCC after complete ablation of radiofrequency ablation (RFA).

Methods: Between Feb 2002 and Dec 2009, patients with BCLC stage 0 and A who received RFA with complete ablation in Kaohsiung Chang Gung Memorial Hospital were included. Baseline characteristic, genotype and viral load were obtained. The recurrent patterns of HCC were classified as local (same segment), distant (other segment), early $(<2$ years) and late ( $>2$ years). Disease-free survival (DFS) rates were analyzed by the Kaplan-Meier curves, and differences between survival curves were statistically compared by log-rank test.

Results: The 1-, 3- and 5-year survival rates were 99\%, $78.4 \%$ and $66.6 \%$, respectively. A total of 105 patients (M/F: $82 / 23$, mean age: $60.2 \pm 9.8$ years) were enrolled. Overall, $1-$, 3 - and 5-year DFS rates of high ( $>10000 \mathrm{IU} / \mathrm{ml})$ and low (s $10000 \mathrm{IU} / \mathrm{ml}$ ) viral load were $64.2 \%, 36.8 \%$ and $24.7 \%$, and $72.5 \%, 56.8 \%$ and $50.6 \%$, respectively ( $\mathrm{p}=0.016)$. There were no significant difference of DFS in tumor size, tumor number, genotype, serum AFP, AST, ALT, albumin, bilirubin, platelet, AST/platelet ratio index and AST/ALT ratio. Multivariate analysis showed high viral load is the independent factor associated with recurrence [HR (95\% C.I.)=1.89 (1.08 3.30)]. In late and distant recurrence, high viral load also the independent factor in recurrence, HR was 2.878(1.050-7.886) and 2.851(1.427-5.695), respectively.

Conclusions: High viral load of HBV infection is associated with overall, late and distant recurrence of early stage HCC after complete RFA.
PE-058
Combination Transarterial Chemoembolization and Radiofrequency Ablation Therapy for Early Hepatocellular Carcinoma: A Propensity Score Analysis Myeong Jun Song, Sung Won Lee ${ }^{1}$, Do Seon Song ${ }^{1}$, Young June Lee ${ }^{2}$, Ho Jong Chun ${ }^{2}$, Jong Young Choi', Seung Kew Yoon ${ }^{1}$, Nam Ik Han, Sang Wook Choi i, Young Sok Lee ${ }^{1}$, Chang Don Lee ${ }^{1}$
${ }^{1}$ Department of Internal Medicine and ${ }^{2}$ Radiology, College of Medicine, The Catholic University of Korea, Seoul, Korea

Background: The purpose of this study was to evaluate the recurrence of hepatocellular carcinoma and the survival rate of patients who received radiofrequency ablation (RFA) after transarterial chemoembolization (TACE) with that of patients treated with TACE or RFA alone.

Methods: Two-hundred and one patients with HCC were consecutively enrolled at Seoul St. Mary's hospital between Dec, 2004 and Feb, 2010. Inclusion criteria were a single HCC $\leq 5.0 \mathrm{~cm}$ or up to three HCCs $\leq 3.0 \mathrm{~cm}$, with no vascular invasion or extrahepatic metastasis. In all, HCC patients $(n=87)$ who had undergone RFA after TACE and those who received TACE $(n=71)$ or RFA only $(n=43)$ were analyzed. Propensity scores were generated to select from each arm of the study.

Results: Median follow-up periods were 33.3 months (6.8-80.9 months). The recurrence rates at 1,3 , and 5 years in the TACE+RFA and RFA groups were similar $(6,33$, and $54 \%$; and 10,31 , and $48 \%$ respectively); however, those in the TACE group were higher $(17,58$, and $78 \%$, respectively). In the TACE+RFA group, the local recurrence rates were significantly lower compared with the RFA group ( $\mathrm{P}=0.034)$. The overall survival rates at 1,3 , and 5 years in the TACE+RFA group $(98,95$, and $90 \%$, respectively) were superior to those in the TACE or RFA groups $(98,90$, and $83 \%$; 94,84 , and $71 \%$; $\mathrm{P}=0.064,0.011$, respectively). In a multivariate analysis of overall survival using a Cox regression model, combination treatment was a significant independent factor for survival compared to RFA and TACE monotherapy (HR, 0.371; 95\% CI, 0.141-0.973; $\mathrm{P}=0.0371, \mathrm{HR}, 0.476 ; 95 \% \mathrm{CI}, 0.230-0.986 ; \mathrm{P}=$ 0.046 , respectively).

Conclusion: The combination of TACE and RFA is an effective treatment for early-stage HCC and results in lower local recurrence and better overall survival rates than those achieved by RFA or TACE alone. 


\section{PE-059 \\ A Case of Massive Bleeding Hemobilia Occurred in Patients with Hepatocellular Carcinoma}

\section{Hee Yeon Kim}

The Catholic University of Korea College of Medicine, Seoul, Korea

Massive bleeding hemobilia occurs rarely in patients with hepatocellular carcinoma (HCC) without any invasive procedure. Upper gastrointestinal bleeding and abdominal pain with progressive jaundice in cirrhotic patients with HCC were usually thought as variceal bleeding and tumor progression, respectively. We recently experienced a case of massive bleeding hemobilia in a patient with HCC. A 73-year-old man had complained of sudden abdominal pain, jaundice and hematochezia. He had alcohol-related cirrhosis and a history of variceal bleeding. He had been diagnosed with HCC 1 year ago, and had been treated with transarterial chemoembolization periodically. Sudden severe right upper abdominal pain occurred, and subsequently subsided with the onset of hemotochezia. Total bilirubin level was elevated compare with the level of last follow up. Computed tomography showed bile duct thrombosis spreading in the intrahepatic and extrahepatic ducts, while duodenoscopy revealed bleeding from an ampulla of Vater. Hemobilia could be one of the causes of massive gastrointestinal bleeding in cirrhotic patients with HCC accompanied by sudden abdominal pain and abrupt elevation of bilirubin level.

\section{PE-060 \\ Radiological Outcome and Safety of Stereotactic Body Radiotherapy in Patients with Hepatocellular Carcinoma: Comparison with 3-dementional Conformal Radiotherapy \\ Jihyun Kim, Hong Soo Kim, Kyung-Hee Hyun, Yun Nah Lee, Sae Hwan Lee, Soung Won Jeong, Sang Gyune Kim, Jae Young Jang, Young Seok Kim, Boo Sung Kim \\ Department of Internal Medicine, Soonchunhyang University College of Medicine, Cheonan, Korea}

Background: With advanced radiotherapy techniques, local tumor control rate can be improved in radiotherapy of hepatocellular carcinoma (HCC). The aim of this study was to investigate the radiological response of three-dimensional conformal RT (3D-CRT) and stereotactic body radiotherapy (SBRT).

Methods: From JAN 2010 to JUN 2012, patients with HCC who received radiotherapy for local tumor control in Soonchunhyang University Cheonan hospital were retrospectively enrolled for the evaluation of tolerability and treatment response. The patients were divided into two groups; 3D CRT group and SBRT group. Treatment response was assessed by RECIST 1.1.

Results: Twenty-five patients with 31 lesions were analyzed. Fourteen patients were BCLC stage C. Eight patients with 10 lesions were treated with 3D-CRT and 17 patients with 21 lesions were treated with SBRT. The mean target tumor size ( $4.2 \pm 5.16 \mathrm{~cm}$ vs. $1.59 \pm 0.86 \mathrm{~cm}, P=0.146)$ and number of tumors ( $1.29 \pm 0.76$ vs. $1.24 \pm 0.44, P=0.838)$. Total radiation dose was significantly higher in SBRT group than 3D-CRT group $(39.96 \pm 10.83$ Gy vs. $52.06 \pm 4.93 \mathrm{~Gy}, P=$ 0.016 ). The mean follow-up period after radiotherapy was $7.1 \pm 5.18$ months. Complete response, partial response, stable disease, and progressive disease were in $0 \%, 0 \%, 50 \%$, and $50 \%$ in 3D-CRT group and in 53\%, 17\%, 12\% and $18 \%$ in SBRT group, respectively. The disease control rate was $50 \%$ in 3D-CRT group and 82\% in SBRT group, however statistically not significant $(P=0.156)$. Grade 1 nausea and uncontrolled ascites were noted in 1 patient each who were treated with 3D-CRT and grade 2 nausea and abdominal pain were developed in 1 patient each who were treated with SBRT, respectively.

Conclusions: SBRT seem to be more effective and safe than 3D-CRT for local tumor control in patients with HCC.

\section{PE-061 \\ Identifying the Optimal Criteria for Radiotherapy in Patients with Intermediate and Advanced Hepatocellular Carcinoma}

\section{Seok Hyun Son}

Incheon St. Mary's Hospital, College of Medicine, the Catholic University of Korea, Incheon, Korea

Purpose: We evaluated the clinical outcomes of radiotherapy (RT) for patients with Barcelona Clinic Liver Cancer (BCLC) intermediate and advanced stage hepatocellular carcinoma (HCC) and established the optimal criteria for RT in these stages.

Methods and Materials: A total of 103 patients were enrolled in this study. All patients received RT delivered using the TomoTherapy Hi-Art system (TomoTherapy Inc., Madison, WI, USA), at Incheon St. Mary's Hospital and Seoul St. Mary's Hospital between March 2006 and February 2012. The planning target volume (PTV) was $330.1 \pm 275.1 \mathrm{~cm} 3$, and the non-target normal liver (NTNL) volume was $1209.7 \pm 426.9$ cm3. The dose per fraction to the PTV was 1.8-5 Gy, and the total dose was 40-60 Gy (median, $50 \mathrm{~Gy}$ ). We evaluated the factors associated with the deterioration of hepatic function and the local progression-free survival (PFS) in order to identify the optimal criteria for RT.

Results: A PTV of $225 \mathrm{cc}$, a total dose of $60 \mathrm{~Gy} 10$, and an NTNL-VBED20 of $40 \%$ were identified as factors associated 
with the deterioration of hepatic function and local PFS. On the basis of these factors, patients were divided to a favorable group or unfavorable group. The differences in median local PFS, overall survival, and the incidence rate of deteriorated hepatic function between the 2 groups were 10.4 months, 11.2 months, and $66.2 \%$, respectively, all of which were statistically significant ( $\mathrm{p}<0.001$ in each case).

Conclusions: We suggest that the optimal criteria for RT in patients with BCLC intermediate and advanced stage HCC were a PTV $\leqq 225 \mathrm{cc}$, a total dose $>60$ Gy10, and an NTNLVBED $20 \leqq 40 \%$.

\section{PE-062 \\ Survival Outcomes and Prognostic Factors after Stereotactic Body Radiation Therapy for Small Hepatocellular Carcinoma

\author{
Sang Min Yoon ${ }^{1}$, Jinhong Jung ${ }^{1}$, Ju Hyun Shim ${ }^{2}$, \\ Kang Mo Kim², Young-Suk Lim², Han Chu Lee ${ }^{2}$, \\ So Yeon Kim ${ }^{3}$, Jin-hong Park ${ }^{1}$, Jong Hoon Kim ${ }^{1}$ \\ Departments of ${ }^{1}$ Radiation Oncology, \\ ${ }^{2}$ Gastroenterology, ${ }^{3}$ Radiology, Asan Medical Center, \\ University of Ulsan College of Medicine, Seoul, Korea
}

Background: Stereotactic body radiation therapy (SBRT) has been an emerging non-invasive local treatment option for patients with hepatocellular carcinoma (HCC) when established curative treatment modalities cannot be applied. In the present study, we report our clinical experiences regarding to SBRT for small, unresectable HCC and evaluate the efficacy and clinical outcomes of this highly sophisticated treatment modality.

Methods: A registry database of 165 patients (178 lesions) treated with SBRT for primary or recurrent HCC between March 2007 and December 2011 were analyzed retrospectively. A dose of 10-20 Gy (median, 15 Gy) per fraction was given over 3-4 consecutive days to a total dose of 30-60 Gy (median, 45 Gy). The probability of cumulative survival was calculated according to the Kaplan-Meier method and compared statistically using log-rank test. Univariate and multivariate analysis were performed using a Cox proportional hazards models.

Results: The median follow-up was 25.3 months (range 1.8-68.6 months). Cumulative incidence of local recurrence rates at 3 years was $9.4 \%$. Overall survival (OS) and intrahepatic progression-free survival (IHPFS) rates at 3 years were $59.0 \%$ and $22.3 \%$, respectively. The incidence of local recurrence was significantly related with viable tumor size (tumor size $<3 \mathrm{~cm}$ vs. $\geq 3 \mathrm{~cm}$ : $5.2 \%$ vs. $28.3 \%$ at 3 years, $\mathrm{p}<0.001$ ). Multivariate analysis revealed that performance status, viable tumor size, and number of prior locoregional treatments were independent predictors of OS. Number of prior locoregional treatments was also independent prognostic factor in predicting IHPFS.
Conclusions: SBRT for small HCC appears to be an effective non-invasive treatment modality for the local management of this tumor. These results suggested that SBRT can be a good alternative modality for the treatment of small HCCs that are unsuitable for surgical resection or local ablative therapy. To determine the accurate efficacy and impact on patients' survival, well-designed prospective investigations are needed.

\section{PE-063 \\ Normalization of Alpha-Fetoprotein Level in Patients with Hepatocellular Carcinoma after Stereotactic Body Radiation Therapy: Prognostic Surrogate of Progression and Survival}

Jinhong Jung, Ju Hyun Shim, Kang Mo Kim, Young-Suk Lim, Han Chu Lee, So Yeon Kim, Jin-hong Park, Jong Hoon Kim

Asan Medical Center, University of Ulsan College of Medicine, Seoul, Korea

Background: The measurement of alpha-fetoprotein (AFP) is useful in the detection of recurrence and in the prediction of survival in patients with hepatocellular carcinoma (HCC) after locoregional or systemic treatment. However, significance of the AFP normalization in HCC patients after stereotactic body radiation therapy (SBRT) had not been evaluated. We investigated the prognostic value of normalization of AFP level for overall survival and progressionfree survival in patients with HCC after SBRT.

Methods: We retrospectively reviewed 165 HCC patients who were treated with SBRT between March 2007 and December 2011 at a single institution. Of these, 77 patients with pre-SBRT AFP level higher than $20 \mathrm{ng} / \mathrm{mL}$ were analyzed. A dose of 10-20 Gy per fraction was given over 3-4 consecutive days, resulting in a total dose of 30-60 Gy. AFP normalization was defined as decrease to $\leq 20 \mathrm{ng} / \mathrm{mL}$ within 3 months after completion of SBRT. Survivals were calculated according to AFP normalization or not.

Results: Thirty-seven (48.1\%) of the 77 patients showed AFP normalization (normalized group). In the other 40 patients (51.9\%), AFP levels did not decrease to $\leq 20 \mathrm{ng} / \mathrm{mL}$ (non-normalized group). No significant difference was observed in patient characteristics between both groups. Overall survival rate at 3 years was $62.0 \%$ and $44.0 \%$ (P = 0.023 ), and progression-free survival rate at 3 years was $27.9 \%$ and $12.0 \%(\mathrm{P}=0.019)$ in the normalized and nonnormalized group, respectively. Intrahepatic recurrence-free survival and distant metastasis-free survival were significantly superior in normalized group than non-normalized group. However, local recurrence-free survival and tumor response were not significant different between these two groups. 
Conclusions: AFP normalization in patients with HCC 3 months after SBRT is a prognostic surrogate for overall survival and progression-free survival. AFP level measurement should be considered as essential tumor marker follow-up especially in patients with elevated AFP level before SBRT.

\section{PE-064 \\ Benefits of Stereotactic Body Radiotherapy after Incomplete Transcatheter Arterial Chemoembolization in Inoperable Hepatocellular Carcinoma \\ Eun Kyung Paik, Mi-Sook Kim, Won Il Jang \\ Department of Radiation Oncology, Korea Cancer Center Hospital, Korea Institute of Radiological and Medical Sciences, Seoul, Korea}

Background: To analyze the effects of additional stereotactic body radiotherapy (SBRT) after incomplete transcatheter arterial chemoembolization (TACE) in inoperable hepatocellular carcinoma (HCC), the treatment outcomes of SBRT and those of other treatment modalities following TACE were compared retrospectively.

Methods: Between January 2006 and December 2008, 497 patients with HCC were treated with TACE as a primary treatment. The following eligibility criteria were applied; (1) single tumor, (2) tumor size $\leq 10 \mathrm{~cm}$, (3) no extrahepatic metastases, (4) Child-Pugh score $\leq 7$, (5) no major vessel invasion, (6) Eastern Cooperative Oncology Group performance status $\leq 2$. The exclusion criteria were as follows; (1) diffuse infiltrative tumor type, (2) tumor occupying $\geq 2 / 3$ of the liver volume, (3) liver cirrhosis-associated complications, (4) severe co-morbidity, (5) previous radiation therapy to the upper abdomen, (6) presence of other malignancies within 5 years. 104 patients were evaluated for TACE failure. Complete response was shown in 19 patients (complete TACE group), and incomplete in 85 patients. Among the 85 patients, 39 had additional SBRT (TACE+SBRT group), and 46 received repeated TACE (incomplete TACE group).

Results: The patient characteristics were similar among the three groups, except that the complete TACE group had smaller tumors compared to other groups. With a median survival of 34 months, overall survival (OS) at 2 years for complete TACE, incomplete TACE and TACE+SBRT groups were $84.2 \%, 45.7 \%$, and $76.8 \%$, respectively, and OS at 5 years were $47.4 \%$, $28.3 \%$, and $63.5 \%$, respectively. Compared with the incomplete TACE group, TACE+SBRT and complete TACE groups showed significantly improved survival rates $(\mathrm{p}=0.006$ and $\mathrm{p}=0.023$, respectively). Survival in complete TACE and TACE+SBRT groups appeared to be statistically equivalent $(\mathrm{p}=0.948)$.

Conclusions: A significantly improved survival benefit was found in the TACE+SBRT group compared to the incomplete TACE group. Therefore, SBRT is strongly recommended for patients with incomplete TACE in inoperable HCC.

\section{PE-065 \\ Significance of an Increase in the Child-Pugh Score after Radiotherapy in Patients with Unresectable Hepatocellular Carcinoma \\ Seok Hyun Son, Hong Seok Jang, In-Young Jo, Byung Ock Choi, Jeong Won Jang, Seung Kew Yoon, Chul Seung Kay \\ Department of Radiation Oncology, Incheon St. Mary's Hospital, College of Medicine, the Catholic University of Korea, Incheon, Korea}

Background: We attempted to analyze the effects of an increase in the Child-Pugh (CP) score on the overall survival of patients with unresectable hepatocellular carcinoma (HCC) after radiotherapy (RT).

Methods: From March 2006 to February 2012, 103 patients received RT using the TomoTherapy Hi-Art at Incheon St. Mary's Hospital and Seoul St. Mary's Hospital. The dose per fraction was 1.8-5 Gy, and the total dose was 40-60 Gy (median, $50 \mathrm{~Gy}$ ). We considered an increase of at least 2 points in the CP score within 3 months after RT to be clinically important radiation-induced hepatic toxicity and analyzed the effects of an increased CP score on overall survival.

Results: The median follow-up duration was 11.6 months (range, 3.5-85.3 months). The median survival time was 11.6 months. In multivariate analysis, an increase in the CP score after RT was found to be a statistically significant factor ( $\mathrm{p}=$ 0.001 ). In a comparison of cases with and without an increase in the CP score, there was an 11.0-month difference in the median survival time (6.9 vs. 17.9 months), and the relative risk of mortality was 2.1 .

Conclusions: An increase of at least 2 points in the CP score within 3 months of RT completion is an important on-treatment factor that affects overall survival, and radiation treatment plans should be established to prevent such an increase. 


\section{PE-066 \\ The Importance of Child-Pugh Score Maintenance in Cirrhotic Hepatocellular Carcinoma Patient after Radiotherapy: Aspect of Gastro-duodenal Complication}

Jeong Il Yu, Ju-Yeon Cho, Hee Chul Park, Do Hoon Lim, Geum-Youn Gwak, Seung Woon Paik,

Samsung Medical Center, Sungkyunkwan University, Seoul, Korea

Background: We designed the present study to evaluate the prevalence of gastro-duodenum (GD) complication and which factors attribute to develop symptomatic GD toxicity after acceptable dose of radiotherapy (RT) in cirrhotic patients with perihilar hepatocellular carcinoma (HCC).

Methods: We performed this retrospective study of cirrhotic 40 perihilar HCC patients treated with RT from August 2008 to September 2010. Esophagogastroduodenoscopy was performed as planned before and after RT. It performed earlier if the patients complained severe symptoms after RT. The maximum point dose of GD was about 40 to 45.6 Gy ( 2 Gy equivalent, $\alpha / \beta=4$ ). GD complication was scored using the Common Terminology Criteria for Adverse Events (CTCAE; version 3.0). Child-Pugh score was assessed at 3 months after completion of RT and compared with score obtained before RT.

Results: After RT, GD complication was observed in 22 patients (55.0\%). Among them, 12 patients (30.0\%) were needed medical interventions. Despite there was no significant factor related with the prevalence of all grade GD complication, but symptomatic (grade II or more) GD complication was significantly related with Child-Pugh score change after RT (P = 0.002).

Conclusions: Considerable portion of GD complication were developed in cirrhotic perihilar HCC after acceptable dose RT. In the aspect of GD toxicities, the liver function maintenance after RT might be also important.

\section{PE-067 \\ Prognostic Value of Alpha-Fetoprotein \\ Response after Radiotherapy Plus Transarterial Chemoembolization for Hepatocellular Carcinoma with Portal Vein Tumor Thrombosis}

\author{
Jin-hong Park ${ }^{7}$, Yuri Jeong ${ }^{1}$, Sang Min Yoon ${ }^{1}$, \\ Jinhong Jung ${ }^{1}$, So Yeon Kim², Ju Hyun Shim ${ }^{3}$, \\ Kang Mo Kim ${ }^{3}$, Young-Suk Lim³, Han Chu Lee ${ }^{3}$, \\ Jong Hoon Kim ${ }^{1}$ \\ Departments of ${ }^{1}$ Radiation Oncology, ${ }^{2}$ Radiology, and \\ ${ }^{3}$ Gastroenterology, Asan Medical Center, University of \\ Ulsan College of Medicine, Seoul, Korea
}

Background: To investigate the value of alpha-fetoprotein (AFP) response for the prediction of radiologic response and survival rates in the patients with hepatocellular carcinoma (HCC) invading portal vein who received 3-dimensional conformal radiotherapy (3D-CRT) and transarterial chemoembolization (TACE).

Methods: We retrospectively reviewed 154 HCC patients with portal vein tumor thrombosis (PVTT) and elevated AFP level ( $>20 \mathrm{ng} / \mathrm{mL}$ ) that underwent 3D-CRT and TACE as initial treatment between August 2002 and August 2008. The $3 \mathrm{D}-\mathrm{CRT}$ volume included PVTT, with $2 \mathrm{~cm}$ margin to adjacent HCC. TACE was done before and after radiotherapy to manage intrahepatic primary HCC. AFP was checked 1 month after radiotherapy, and AFP response was defined as an AFP reduction of more than $20 \%$ from the initial level. Radiologic response, overall survival (OS), and progression-free survival (PFS) rates were estimated from the date of start of treatment and compared between AFP responders and non-responders.

Results: The median follow-up period was 11.1 months (range, 3.1-82.7 months). Initial AFP level was $400 \mathrm{ng} / \mathrm{mL}$ or less in $52(33.8 \%)$ patients. Of the 154 patients, $99(64.3 \%)$ were AFP responders and 55 (35.7\%) were AFP nonresponders. At 6-month radiologic response evaluation, 54 (35.1\%) patients achieved objective responses (complete or partial response), and 100 (64.9\%) were stable or progressive diseases. The objective response rate was higher in AFP responders compared with AFP non-responders (48.5\% vs. $10.9 \%, \mathrm{p}<0.001$ ). OS and PFS were also higher in AFP responders than in non-responders (median OS 15.5 months vs. 5.8 months, $\mathrm{p}<0.001$; median PFS 11.1 months vs. 3.5 months, $\mathrm{p}<0.001$ ).

Conclusions: AFP response was significant predictive factors for radiologic response. Furthermore, AFP response was also significant for OS and PFS. AFP evaluation after combined radiotherapy and TACE seems to be a useful tool in predicting clinical outcomes for HCC patients with PVTT. 


\section{PE-068 \\ Duodenal Ulcer Bleeding Associated with Coil Migration after Hepatic Arterial Infusion Chemotherapy and Tomotherap \\ Chung-Hwa Park', Hee Yeon Kim ${ }^{7}$, Jin Su Kim', Ho Jong Chun ${ }^{2}$, Jong Young Choi ${ }^{1}$, Seung Kew Yoon ${ }^{7}$, Si Hyun Bae ${ }^{1+}$ \\ Department of ${ }^{1}$ Internal Medicine and ${ }^{2}$ Radiology' Medical College, The Catholic University of Korea, Seoul, Korea}

The risk of gastrointestinal bleeding is known to be increased in HCC cases with underlying cirrhosis. Furthermore, inappropriate infusion of chemotherapeutic or embolizing agent into gastric artery, vessel spasm during catheterization, or stress ulcer may increase the risk of bleeding in HCC patients. Recently, as more advanced radiation therapies became feasible for unresectable HCC patient, cases of radiation induced gastrointestinal injury, which may be associated with increased mucosal friability are reported.

Here, we report a rare case of duodenal bleeding in a patient with recurrent HCC. A forty-eight year-old male patient presented with upper gastrointestinal bleeding a year after receiving tomotherapy and hepatic artery infusion chemotherapy for a recurrent HCC in segment 1. On endoscopic evaluation, duodenal ulcer bleeding with an exposed micro-coil was observed. The duodenal ulcer seemed to have developed by the micro-coil which had migrated and penetrated the friable duodenal mucosa. The microcoil was implanted in the gastroduodenal artery during hepatic arterial infusion catheter insertion 8 months prior to the event, and the mucosa seemed to have become friable after radiation therapy eventually causing gastrointestinal bleeding.

\section{PE-069 \\ Therapeutic Effect of High-Intensity Focused Ultrasound Ablation and TACE Combination for Hepatocellular Carcinoma - Single Center Experience}

Hyun Jin Kim, Se Hyun Cho, Joon Yeol Han

Division of Gastroenterology and Hepatology, Department of Internal Medicine, The Catholic University of Korea, Seoul, Korea

Background: Hepatocellular carcinoma (HCC) is the prevalent cancer in Asia, but the choice of treatment modality should be considered cancer staging and the liver function. The modalities of curative treatment for HCC are resection, liver transplantation, radiofrequency ablation and percutaneous ethanol injection therapy. HIFU is a non-invasive treatment method that induces coagulation necrosis of the target lesion by ultrasound waves, described as clinical trial on 2009 guideline for the management of HCC of KLCSG and NCC, Korea. HIFU and TACE combination therapy for HCC has reported good outcome in many studies. In this retrospective study, we assessed the treatment outcomes of TACE with HIFU combination therapy for HCC in single center.

Methods: We review the medical records of all patients with diagnosed HCC and treated HIFU and TACE, retrospectively. Between January 2006 and December 2010, combination therapy was performed in 68 patients with HCC (50 men and 18 women, mean age $57.4 \pm 7.5$ years, ECOG 0 or 1 ), and underlying chronic liver disease by alcohol (7), HBV (55), HCV (5), etc (1), 66 patients was diagnosed with liver cirrhosis. In evaluation for liver function, we applied to ChildPugh classification and MELD score (5(A); 23, 6(A); 30, 7(B); 15 and $9.6 \pm 3.0$ ). For evaluation of cancer staging, we applied to BCLC and modified UICC staging system (BCLC; 0 (4), $\mathrm{A}(20), \mathrm{B}(34), \mathrm{C}(10)$ and stage I(5), II(42), III(14), IVA(3), $\operatorname{IVB}(4))$.

Results: The mean size of mass was $3.66 \pm 2.20(<2 \mathrm{~cm} ; 10$, $2-3 \mathrm{~cm} ; 16,>3 \mathrm{~cm} ; 42)$, the types of mass were nodular (63), infiltrative (3), mixed (2). The tumor response for targeted mass was assessed according to mRECIST, 24 patients had a CR, 22 patients had a PR, 7 patients had a SD, 15 patients had a PD. The PD group included recurrence from 3 months to 2 years.

Conclusions: HIFU and TACE combination therapy is effective for control of unresectable HCC. Non curative therapy like TACE with HIFU should be trying for survival for HCC.

\begin{tabular}{ll}
\hline Characteristics of patient & \\
\hline Patient No & 68 \\
Gender (M:F) & $50: 18$ \\
Etiology (alcohol/HBV/HCV/etc) & $7 / 55 / 5 / 1$ \\
Mean age (years) & $57.4 \pm 7.5$ \\
Child-Pugh classification (A/B) & $53 / 15$ \\
MELD score & $9.6 \pm 3.0$ \\
Mean Serum AFP (ng/ml) & $558.3 \pm 1717.6$ \\
Mean tumor size (cm) & $3.66 \pm 2.20$ \\
BCLC stage (0/A/B/C) & $4 / 20 / 34 / 10$ \\
Modified UICC stage (I/II/III/IVA/IVB) & $5 / 42 / 14 / 3 / 4$ \\
\hline
\end{tabular}

\section{PE-070 \\ Radiotherapy for Spinal Metastases from Hepatocellular Carcinoma \\ Chi Hwan Choi, Jinsil Seong, Hae Ok Pyun \\ Department of Radiation Oncology, Yonsei University College of Medicine, Seoul, Republic of Korea}

Background: The spine was the most common site of bone metastases from hepatocellular carcinoma (HCC). Spinal metastases often severely limit the quality of life due to severe pain and neurological deficits. The purpose of the study was 
to evaluate the palliative effect of radiotherapy (RT) and to identify predictive factors of survival in patients with spinal metastases from HCC.

Methods: A retrospective analysis was performed upon 192 patients with spinal metastases from HCC who received RT between March 1992 and February 2012. Total radiation doses were recalculated and normalized to obtain biologic effective dose (BED) as $\alpha / \beta$ ratio $=10 \mathrm{~Gy}$. The total BED ranged from 3.9 to 78 grays $\left(\mathrm{Gy}_{10}\right)$ (median, $\left.44.2 \mathrm{~Gy}_{10}\right)$ and was focused on the involved spine. Pain response was assessed using the criteria of International Bone Metastases Consensus Group (IBMCG). Survival was defined as the time from the date when spinal metastases were first diagnosed to the date of death or the last follow-up appointment. Predictive factors associated with overall survival was investigated by multivariate analyses.

Results: Of 192 patients with spinal metastases from HCC, a complete response (CR) from RT occurred in 41 (21.4\%) patients, partial response (PR) in 146 (76.0\%) patients, stable pain in $3(1.6 \%)$ patient, and pain progression (PP) in $2(1.0 \%)$ patients. Overall pain response (CR + PR) to palliative RT occurred in 187 patients $(97.4 \%)$. The overall survival rate at 1 and 2 years was $18.1 \%$ and $6.3 \%$, respectively, with a median survival time of 4.5 months. In multivariate analysis, longer survival was associated with good performance status, Child-Pugh classification (A or B), controlled primary HCC, absence of visceral metastases, higher BED ( $>45 \mathrm{~Gy}_{10}$ ), a longer interval between diagnosis of spinal metastases and starting treatment of spinal metastases (days), and presence of complete pain response (CR).

Conclusions: RT provides effective palliation for patients with painful spinal metastases from HCC. Identification of predictive factors associated with good survival may allow determination of an optimal radiotherapy schedule for patients with spinal metastases from HCC

\section{PE-071 \\ Could Bile Duct Invasion Be Considered as the Same as Vascular Invasion Be in Staging of Hepatocellular Carcinoma?}

Yong Keun Park, Bong-Wan Kim, Weiguang Xu, Hee-Jung Wang

Department of Surgery, Ajou University School of Medicine, Suwon, Korea

Background/Aim: Hepatocellular carcinoma (HCC) presenting as bile duct invasion (BDI) is rare, and the independent prognostic value of this finding is unclear. According to Japanese TNM staging system for HCC, BDI is considered to carry the same prognostic significance as portal vein invasion (PVI) and hepatic vein invasion (HVI). However, AJCC TNM and the Barcelona clinic liver cancer staging system contain no mention of BDI. The current study mainly aimed to evaluate the predictive power of BDI in a cohort of patients with HCC who underwent surgical treatment.

Materials and Methods: 728 consecutive HCC patients underwent surgical treatment from 1994 to 2011 at our institution were enrolled in this study. Clinicopathological and oncologic outcome data were retrospectively reviewed.

Results: 28 patients with combined HCC and cholangiocarcinoma were excluded in this analysis. Overall and diseasefree 5 year survival rates of the remaining 700 patients were $63.3 \%$ and $42.6 \%$. After a median follow-up of 33 months, 379 patients (52\%) developed cancer recurrence and 261 patients $(35.8 \%)$ died. 42 cases (6\% of 700 ) had HCC with BDI, and those 5 year survival rate were $48 \%$. Independent risk factors for recurrence included tumor size $>5 \mathrm{~cm}$, microvascular invasion, PVI, HVI, intrahepatic metastasis, microscopic positive resection margin and cirrhosis. Tumor size $>5 \mathrm{~cm}$, microvascular invasion, intrahepatic metastasis, microscopic positive resection margin, tumor rupture and cirrhosis were significantly associated with decreased overall survival. However, BDI had no significant predictive value in multivariate analysis. Both AJCC TNM and Japanese TNM had good discriminating ability in the whole cohort, but the latter lost its prognostic power in a cohort of 42 patients who had HCC with BDI.

Conclusions: BDI was not revealed to be independent risk factor in patients with HCC who underwent surgical treatment. Japanese TNM staging system that considers BDI as the same as vascular invasion seems to be less appropriate for stratifying HCC patients, especially with BDI.

\section{PE-072 \\ Clinical Applicability of Deformable Image Registration in Re-Irradiation of Hepatocellular Carcinoma \\ Dong Soo Lee ${ }^{1,2}$, Joong Ryul Woo², Jun Won Kim², Jinsil Seong ${ }^{2}$ \\ ${ }^{1}$ Department of Radiation Oncology, Seoul St. Mary's \\ Hospital, the Catholic University of Korea, Seoul, \\ ${ }^{2}$ Department of Radiation Oncology, Severance \\ Hospital, Yonsei University Health System, Seoul, Korea}

Purpose: In locally advanced hepatocellular carcinoma (HCC), additional radiation is often required to facilitate local control after previous irradiation of primary tumor. However, safety issue is a major concern. This study aimed to evaluate whether deformable image registration (DIR) method would be clinically applicable to safe delivery of re-irradiation.

Materials and Methods: Between August 2010 and March 2012, 12 eligible HCC patients had received full tolerable doses of re-irradiation using helical tomotherapybased intensity modulated radiation therapy. The median total prescribed radiation dose at first and re-irradiation 
course was 50 Gy (range, 36-60) and 50 Gy (range, 36-58.42), respectively. Most (11/12) of re-irradiation was administered to previously irradiated or marginal areas. Dose summation results were reproduced using DIR by both rigid and deformable registration methods and organs-at-risk (OARs) doses were evaluated. Treatment outcomes were also assessed.

Results: Thirty-six dose summation indices were obtained for three OARs (bowel, duodenum and stomach dose in each patient). There was no statistical difference between two different types of DIR methods (rigid and deformable type) in terms of calculated (by DIR) $\Sigma \mathrm{D}_{0.1 \mathrm{cc}}, \Sigma \mathrm{D}_{1 \mathrm{cc}}, \Sigma \mathrm{D}_{2 \mathrm{cc}}$ and $\Sigma \mathrm{D}_{\max }$ in each OAR. However, 12 (33.3\%) indices were finally proved to be useful in availability of utilizing DIR method in summated dose prediction. The median total mean remaining liver doses $\left(\mathrm{M}_{\mathrm{RLD}}\right)$ in rigid and deformable type registration were not statistically different in the entire cohorts $(p=$ 0.248 ), but large difference of $M_{R L D}$ was observed when there was a significant difference in spatial liver volume change between the first and second course of RT. Elevation of ChildPugh score $\geq 2$ after re-irradiation was strongly associated with performing transarterial chemoembolization (TACE) as the combined treatment modality at re-irradiation. One duodenal ulcer perforation developed 20 months after re-irradiation.

Conclusions: In re-irradiation of HCC, DIR-based radiation dose summation may be helpfully utilized in terms of reducing gastrointestinal toxicities after appropriate cohort selection and watchful interpretation. Careful visual confirmation of high risk regions and selective use of DIR methods are crucial to enhance radio-therapeutic ratios.

\section{PE-073 \\ Management of End-Stage Hepatocellular Carcinoma by Dietary Supplement, ONCOXIN $^{\circledR}$ : Clinical Implication in the Management of Advanced Cancer in Developing and Resource-Constrained Countries}

Ayub Al-Mamun, Mamun Al-Mahtab', Sheikh Moammad Fazle Akbar', Helal Uddin', Lovely Begum ${ }^{4}$, Nasrin Zahan', Ayub Al Mamun?, Salimur Rahman ${ }^{7}$

${ }^{1}$ Department of Hepatology, Bangabandhu Sheikh Mujib Medical University, Dhaka, Bangladesh; ${ }^{2}$ Department of Medical Sciences, Toshiba General Hospital, Tokyo, Japan; ${ }^{3}$ Clinical Research Organization, Dhaka, ${ }^{4}$ Catalysis South Asia Operations, Dhaka, Bangladesh

Background: Management of end-stage hepatocellular carcinoma (HCC) is not only a medical challenge, but also has profound socio-economical concerns. Dietary supplements have shown some promise to increase quality of life in patients with liver cirrhosis and early HCC. However, the impact of dietary supplements has not been assessed in end-stage HCC.

Materials and Methods: The study (NTC01392131) presented here assessed if dietary supplement has any impact on survival and quality of life in end-stage HCC patients at Bangladesh. 19 patients with end stage HCC were enrolled after physicians, surgeons and oncologists abandoned them completely with 'nothing to do' approach. They had multiple HCC with features of decompensation. They received $25 \mathrm{ml}$ of ONCOXIN ${ }^{\circledR}$, a dietary supplement, containing high levels of amino acids (glycine; $2000 \mathrm{mg}$; glucosamine; $2000 \mathrm{mg}$; malic acid, 1200 mg; arginine, 640 mg; cystine, $204 \mathrm{mg}$ with zinc, green tea extract and folic acid), twice daily along with tablet ONCOXIN $^{\circledR}$ twice daily for 3 months. A control group of 10 patients with end-stage HCC were managed conservatively without any additive therapy. All patients were followed up until death.

Results and Discussion: 7 of 19 patients receiving ONCOXIN $^{\circledR}$ survived for more than 3 months and 4 of them were alive for more than 6 months. On the other hand, all patients receiving no ONCOXIN ${ }^{\circledR}$ survived for less than 2 months. The extent of pain, appetite, and other features of quality of life appeared to be better in patients receiving ONCOXIN ${ }^{\circledR}$ compared to those without ONCOXIN ${ }^{\circledR}$, but significant difference was not seen in this context.

Conclusion: This study provides scientific and ethical basis for undertaking randomized-clinical trial with $\mathrm{ONCOXIN}^{\circledR}$ in end-stage HCC offering treatment strategy for them, which is cheap and compliable at home bearing significant clinical importance in developing countries.

\section{PE-074}

\section{A Randomized, Controlled Phase III Trial of Sorafenib with or without Conventional Transarterial Chemoembolization in Patients with Advanced Hepatocellular Carcinoma (STAH)}

Joong-Won Park ${ }^{7}$, Yoon Jun Kim², Do Young Kim ${ }^{3}$, Jong-Young Choi ${ }^{4}$, Jong Eun Yeon ${ }^{5}$, Sang Young Han ${ }^{6}$, Jae Seok Hwang ${ }^{7}$, Youn-Jae Lee ${ }^{8}$, Jae Youn Cheong ${ }^{9}$, Oh Sang Kwon ${ }^{10}$, Hwi Young Kim ${ }^{11}$, Han Chu Lee ${ }^{12}$, Kwan Sik Lee ${ }^{13}$, Bo Hyun Kim ${ }^{1}$, Seung Woon Paik ${ }^{14}$

${ }^{1}$ National Cancer Center Hospital, ${ }^{2}$ Seoul National University Hospital, ${ }^{3}$ Severance Hospital, ${ }^{4}$ The Catholic University Hospital of Korea, ${ }^{5}$ Korea University Guro Hospital, ${ }^{6}$ Dong-A University Medical Center, ${ }^{7}$ Keimyung University Dongsan Medical Center, ${ }^{8}$ BusanPaik Hospital, ${ }^{9}$ Ajou University Hospital, ${ }^{10}$ Gachon University Gil Hospital, ${ }^{11}$ Boramae Medical Center, ${ }^{12}$ Asan Medical Center, ${ }^{13}$ Gangnam Severance Hospital,

${ }^{14}$ Samsung Medical Center, Seoul, Korea 
Background: The conceptual advantage of a combination of transarterial chemoembolization (TACE) plus antiangiogenic agents is the possible synergistic effect in patients with hepatocellular carcinoma (HCC). Positive results of two phase II studies combining TACE and sorafenib have been reported. The COTSUN study (NCT00919009) showed a promising time to progression in patients with advanced HCC. Two phase III studies of the combination of TACE and a molecularly targeted agent, ECOG 1208 (NCT01004978) and ORIENT (JapicCTI-101354), for intermediate or advanced HCC are ongoing. However, no study shows the additional effect of TACE with sorafenib treatment in patients with advanced HCC, particularly in patients with macrovascular invasion or extrahepatic metastasis.

Methods: STAH is a randomized, multi-center, openlabeled, phase III study in patients with advanced HCC. A total of 338 patients will be randomized (1:1) into 1 of the 2 treatment arms: sorafenib with (arm combination treatment; arm C) or without (arm sorafenib alone; arm S) conventional TACE (cTACE). Randomization will be stratified according to the modified International Union Against Cancer (mUICC) stage (III vs. IV), vascular invasion (none and Vp1-2 vs. Vp3-4 and any other presence), Child-Pugh score (5 vs. 6-7), and serum alpha-fetoprotein level $(\geq 200 \mathrm{ng} / \mathrm{mL}$ vs. $<200 \mathrm{ng} / \mathrm{mL})$. The primary objective is to determine overall survival (OS) in the 2 arms. Assuming a median OS associated with sorafenib treatment of 9.5 months and a median OS associated with combination treatment of 13.0 months, 241 events will provide an $80 \%$ one-sided power with an $\alpha$ value of 0.05 to detect a difference in OS between the 2 groups.

The main inclusion criterion is advanced HCC (mUICC stage III, IVa, IVb) that is indicated for systemic chemotherapy as the treatment of choice according to the Korean guidelines. All eligible patients will take sorafenib (both arms) within 3 days after randomization, while patients in arm $\mathrm{C}$ will undergo the first cTACE within 14 days after randomization, with subsequent cTACE performed as needed according to re-determined criteria. Enrollment has begun on this study (clinical trial number, NCT01829035).

\section{PE-075 \\ Sucessful Salvage of a Patient with Multiple Relapses and Metastatic Hepatocarcinoma with Adjuvant Combination Immunotherapy: A Case Study}

David M. Chan, Nathan R. Chan, Raymond Chang

University of British Columbia and University of Gottingen, Germany

Background: Patients who failed target therapy with multiple relapses and metastatic hepatocarcinoma are preterminal. Aggressive innovative combination Immunotherapy was applied to salvage the patient.
A 62 years Chinese Male with Chronic hepatitis B, genotype $\mathrm{C}$, nucleotide analog treated, HBVDNA negative patient developed hepatocarcinoma in Segment VI and VII in 2002. This was successfully treated with RFA. In April 2009, recurrence of hepatocarcinoma was detected at Segment VI and treated with Cyberknife 64Gy.

Sorenfenib was started but failed to prevent spread of tumor to C4 in July. Cyberknife 175Gy was applied. The patient started on dendritic cell tumor vaccine. He remained well until February 2002 when PET scan showed recurrence of tumor at C4. Salvage procedures started in April.

Method: The patient was started on monthly treatment of Tumorcidal dendritic cells derived from the patient's monocytes through leucophoresis. The monocytes were then cultured with GMCSF and stimulated with various TLR to turn into tumor specific dendritic cells to be given monthly. In addition, Ibandronic acid $6 \mathrm{mg}$ infusion, followed by Interleukin II 3 million iu.subcutaneously monthly and alpha 1 Thymosin $3.2 \mathrm{mg}$ weekly were applied.

Results: After 6 months of treatment with intensive immunotherapy, repeat PET scan in November 2012 showed resolution of the metastatic lesion at $\mathrm{C} 4$ with marked decrease in C-acetate uptake on PET scan. Dendritic cell treatment then reduced to quarterly but the other two treatments continued on.

Conclusion: Tumorcidal dendritic cells, interleukin II stimulated gamma-delta lymphocytes, activated with phosphorylation with Ibandronic acid and enhancement of cytotoxic lymphocytes and Natural Killer cells with Thyrosin all contributed to the success. Adjuvant Immunotherapy combination should be considered as a preventive measure for metastasis and recurrence for hepatocarcinoma patients after primary treatment.

\section{PE-076 \\ Engineered IL12-expressing Mesenchymal Stem Cells Enhance the Tumor Therapeutic Effect of Irradiation in Murine Hepatocellular Carcinoma}

Keun-Yeong Jeong ${ }^{1}$, Eun-Jung Lee ${ }^{1}$, Seung-Hyun Yang ${ }^{1}$, Soo Jin Kim², Young Chul Sung ${ }^{2}$, Jinsil Seong ${ }^{1 *}$

${ }^{1}$ Department of Radiation Oncology, Yonsei University Health System, Seoul, ${ }^{2}$ Division of Molecular and Life

Science, Pohang University of Science and Technology (POSTECH), Pohang, Gyeongbuk, Korea

Background: Irradiation is a standard therapy for many cancers including liver cancer. Recently, irradiation is often used in conjunction with other cancer treatments for efficient treatment of the cancer, such as cancer gene therapy. Mesenchymal stem cell (MSC) has been considered as an ideal delivery vehicle for cancer gene therapies. In this study, we investigated whether intra-tumoral (i.t.) injection of IL12- 
expressing MSCs (MSCs/IL12) would enhance the antitumor effect of irradiation on murine hepatic cancer model.

Methods: Murine hepatic carcinoma $\mathrm{HCa}-1$ showing high resistance to antitumor treatment was used, and heterotopic tumor bearing $\mathrm{C} 3 \mathrm{H} / \mathrm{HeN}$ mice were treated with $10 \mathrm{~Gy}$ single or fractionation irradiation, MSCs/IL12, or combination in tumor growing site. First, tumor growth volume, metastatic effect, and survival rate were evaluated. To investigate mesenchymal stem cell homing effect of irradiation, levels of chemoattractant MCP-1/CCL2 cytokine and MSC marker CD73 were examined. Then, increase of IL12 by MSCs/IL12 homing was also evaluated. To understand underlying mechanism, the level of apoptosis was examined as well as changes in expression of immunological markers: IFN- $\gamma$ and $\mathrm{CD}^{+}$.

Results: Antitumor effect was shown in all experimental groups. In the comparison between the groups, the antitumor effect and survival rate were significantly increased in the combination group compared to other groups $(P<0.05)$. The number of lung nodules by metastasis significantly decreased in the combination group $(P<0.05)$. Immunofluorescence assay of chemo-attractant MCP-1/CCL2 cytokine and MSC marker CD73 indicates that mesenchymal stem cell homing significantly increased in the combination group $(P<0.05)$, and IL12 also significantly increased in the same group $(P<$ $0.05)$. Expression of IFN- $\gamma$ and $\mathrm{CD}^{+} \mathrm{T}$-cell activity significantly increased in the combination group $(P<0.05)$, suggesting an underlying mechanism of antitumor effect. Significantly increased level of apoptosis in the combination group $(P<0.05)$ indicates an enhancement of antitumor effect.

Conclusion: In conclusion, intra-tumoral injection of MSCs/IL12 increases the antitumor effect of radiation. These effects seem through increased homing of MSCs by irradiation as well as IL12-induced CD8 positive cytotoxic T-cell activity. Therefore, MSCs/IL12 may be a more useful strategy for enhancement of cancer radiation therapy.

\section{PE-077 \\ Association between Polymorphisms of PPM1E Gene and Tumor Size of Hepatocellular Carcinoma Patients in Korean Population \\ Min-Su Park ${ }^{1}$, Yoo Shin Choi ${ }^{2}$ \\ ${ }^{1}$ Department of Surgery, School of Medicine, Kyung- Hee University, ${ }^{2}$ Department of Surgery, Chung-Ang University, Seoul, Korea}

Introduction: Hepatocellular carcinoma (HCC) is the most common type of primary liver cancer in Korea. Recently, there is an increasing evidence that polymorphism in genes may has a role in altering the risk of HCC. Protein phosphatase plays a crucial role in biological function and controls nearly every cellular process. The protein phosphatase, $\mathrm{Mg} 2+/ \mathrm{Mn} 2+$ dependent, 1E (PPM1E) inactivates multiple substrates including 5'-AMP activate protein kinases (AMPK) which inhibits the growth and survival of cancer cells. However, no study on the possible genetic association of PPM1E single nucleotide polymorphism (SNP) with HCC has been conducted yet.

Patients and Methods: HCC patients (153 males and 30 females) and healthy individuals (167 males and 224 females) were enrolled in this study. The Age and sex of controls matched those of HCC patients. We selected three exonic SNPs (rs16943333, rs3809724 and rs3809723) in the 3'- UTR of the PPM1E gene.

Results: rs16943333, rs3809724 and rs3809723 of PPM1E were not significant differences between the HCC group and the control group. In the further analysis, we divided HCC patients into two groups according to tumor size, serum AFP level, UICC stage, radiologic morphology and portal vein thrombosis. We found that rs16943333 and rs3809724 in PPM1E were significantly associated with the tumor size. The genotype frequency of rs16943333 was associated with tumor size in the codominant 2 (G/G vs. A/A, p = 0.038, Fisher's exact $\mathrm{p}=0.06, \mathrm{OR}=6.27,95 \% \mathrm{CI}=1.11-35.41$ ), dominant (G/G / G/A vs. A/A, p $=0.014,0 R=2.27,95 \%$ $\mathrm{CI}=1.17-4.41)$ and log-additive models $(\mathrm{p}=0.0058,0 \mathrm{R}-2.16$, $95 \% \mathrm{CI}=1.23-3.79$ ). In the allele frequency analysis, rs16943333 was associated with tumor size $(\mathrm{p}=0.010$, $\mathrm{OR}=2.07,95 \% \mathrm{CI}=1.19-3.59$ ). The genotype frequency of rs3809724 was associated with tumor size in the codominant 2 (C/C vs. T $\mathrm{T}, \mathrm{p}=0.030$, Fisher's exact $\mathrm{p}=0.09, \mathrm{OR}=5.31,95 \%$ $\mathrm{CI}=1.17-24.05)$, dominant $(\mathrm{C} / \mathrm{C} / \mathrm{C} / \mathrm{T}$ vs. $\mathrm{T} / \mathrm{T}, \mathrm{p}=0.030$, $\mathrm{OR}=2.04,95 \% \mathrm{CI}=1.07-3.89)$ and log-additive models ( $\mathrm{p}=$ $0.011,0 R-1.97,95 \% \mathrm{CI}=1.16-3.37)$. In the allele frequency analysis, $\mathrm{rs} 3809724$ was associated with tumor size $(\mathrm{p}=$ 0.023, OR=1.84, 95\% CI=1.09-3.12).

Conclusion: In conclusion, we found that PPM1E polymorphisms were significantly associated with tumor size of HCC patients. In particular, the frequency of A alleles (rs16943333) and T allele (rs3809724) increased in HCC patients with large tumor size.

\section{PE-078 \\ Association of CHKA Polymorphism (rs3794186) with $\alpha$-fetoprotein Levels in Hepatocellular Carcinoma}

Min-Su Park, Sang-Mok Lee, Joo-Ho Chung

Department of Surgery, School of Medicine, Kyung-Hee University, Seoul, Korea

Choline kinase $\alpha$ (CHKA) has been identified to be associated with cancer development and progression. In this study, we investigated whether exonic single nucleotide polymorphisms (SNPs) of the CHKA gene are associated with hepatocellular carcinoma (HCC). Among all SNPs in the 3'-untranslated region (UTR), 5'-UTR and the coding region of CHA, only two SNPs (rs3794186 and rs11481) in the 3'-UTR had a heterozygosity above 0.1 and a minor allele frequency 
above 0.1. Therefore, we selected and assessed these two SNPs (rs3794186 and rs11481) in 189 HCC patients and 194 controls. Genetic data were analyzed using the SNPAnalyzer Pro, SNPStats and Haploview programs. No SNPs of the CHKA gene were found to be associated with the risk of HCC development. Upon analysis of the clinical characteristics of HCC, the genotypic frequency of rs3794186 was significantly associated with serum $\alpha$-fetoprotein (AFP) levels ( $\mathrm{P}=0.022$ in the co-dominant 1 model, $\mathrm{P}=0.0045$ in the dominant model and $\mathrm{P}=0.0052$ in the log-additive model). A significant difference in the allelic frequency of rs3794186 was also observed between the high AFP ( $>200 \mathrm{ng} / \mathrm{ml}$ ) group and the low AFP $(\leq 200 \mathrm{ng} / \mathrm{ml})$ group $[\mathrm{P}=0.009$, odds ratio $(\mathrm{OR})=0.33$, $95 \%$ confidence interval (95\% CI) 0.14-0.75]. The T allele frequency of rs3794186 was lower in the high AFP group (6.6\%) compared to that in the low AFP group (17.8\%). Our results suggest that CHKA SNPs (rs3794186 and rs11481) are not associated with HCC development; however, rs3794186 may correlate with serum AFP levels in HCC.

\section{PE-079 \\ Activation of mTORC1 and Raf/Pyk2 by Growth Factor-Mediated EphA2 is Required for Cholangiocarcinoma Growth and Metastasis}

Mi-Jin Lee Xiang-Dan Cui, Goung-Ran Yu, Yun-Peng Wang, Hua Lee, In-Hee Kim, Dae-Ghon Kim

Division of Gastroenterology and Hepatology, Department of Internal Medicine, Institute for Medical Science, Chonbuk National University Medical School and Hospital, Jeonju, Korea

Background: EphA2 overexpression is frequently accompanied by the loss of its cognate ligand during tumor progression. However, the molecular mechanism of this ligand-independent promotion of tumor by EphA2 remains unclear in highly malignant and fatal cholangiocarcinoma (CC).

Methods: We examined the biological role of EphA2 in tumor growth and metastasis in CC tissues and cells according to the degree of differentiation and we explored the downstream signaling pathways of EphA2.

Results: Growth factor-mediated EphA2 overexpression itself leads to the activation of the mTORC1 and ERK pathways through ligand-independent activation of EphA2 (phosphorylation of S897). An in vitro soft agar assay and in vivo subcutaneous tumor model showed that EphA2 enhanced colony formation and accelerated tumor growth, which seemed to be mainly associated with Akt (T308)/mTORC1 activation. Aberrant expression and activation of EphA2 was also associated with poorer differentiation and higher metastatic ability. Metastatic ability was also enhanced in a mouse lung metastasis model, correlating with Pyk2(Y402)/c-Src/ERK activation in addition to activation of the canonical Raf/MEK/ ERK pathway. The mTORC1 and Raf/Pyk2 pathways also appeared to affect each other.

Conclusions: These results suggest that growth factormediated EphA2 might be involved in tumor growth and metastasis through activation of the mTORC1 and Raf/Pyk2 pathways. Therapeutic strategies that target EphA2 and its downstream effectors may be useful to control CC.

\section{PE-080 \\ SH3RF2 Functions as an Oncogene by Mediating PAK4 Protein Stability}

Tae Woo Kim ${ }^{1,4}$, Yun Kyung Kang ${ }^{2}$, Zee Yong Park ${ }^{3}$, Eunhee Kim ${ }^{4}$, Young Il Yeom ${ }^{1 *}$, Kyung Chan Park ${ }^{1 *}$

${ }^{1}$ Medical Genomics Research Center, KRIBB, ${ }^{2}$ Departments of Pathology, Inje University, Seoul Paik Hospital, ${ }^{3}$ Department of Life Science and Research Center for Biomolecular Nanotechnology, Gwangju Institute of Science and Technology, ${ }^{4}$ College of Biological Sciences and Biotechnology, Chungnam National University, Korea

SH3RF (SH3 domain containing RING finger protein) family members, SH3RF1-3, are multidomain scaffold proteins involved in promoting cell survival and apoptosis. In this report, we show that SH3RF2 is an oncogene product that is overexpressed in human cancers and regulates p21-activated kinase 4 (PAK4) protein. Immunohistochemical analysis of 159 colon cancer tissues showed that SH3RF2 expression levels are frequently elevated in cancer tissues and significantly correlate with poor prognostic indicators, including increased invasion, early recurrence, and poor survival rates. We also demonstrate that PAK4 protein is degraded by the ubiquitin-proteasome system and that SH3RF2 inhibits PAK4 ubiquitination via physical interaction-mediated steric hindrance, which results in the upregulation of PAK4 protein. Moreover, ablation of SH3RF2 expression attenuates TRADD (TNFRSF1A-associated via death domain) recruitment to TNF- $\alpha$ (tumor necrosis factor- $\alpha$ ) receptor 1 and hinders downstream signals, thereby inhibiting NF- $\mathrm{KB}$ (nuclear factorкарраB) activity and enhancing caspase- 8 activity, in the context of TNF- $\alpha$ treatment. Notably, ectopic expression of SH3RF2 effectively prevents apoptosis in cancer cells and enhances cell migration, colony formation and tumor growth in vivo. Taken together, our results suggest that SH3RF2 is an oncogene that may bea definitive regulator of PAK4. Therefore, SH3RF2 may represent an effective therapeutic target for cancer treatment. 


\section{PE-081 \\ HAUL Regulates IL-6-induced JAK2/STAT3 Signaling by Hindering the Inhibitory Function of SOCS3}

Su Jin Oh ${ }^{1,2}$, Tae Woo Kim ${ }^{1}$, Young $/ l$ Yeom $^{2 *}$, Kyung Chan Park ${ }^{1 *}$

${ }^{1}$ Medical Genomics Research Center, KRIBB, Daejeon, Republic of Korea; ${ }^{2}$ Department of Functional Genomics, University of Science and Technology, Daejeon, Korea

Interleukin-6 (IL-6) is a multifunctional cytokine, which activates STAT3 (Signal Transducer Activator of Transcription 3) through gp130 receptor and JAK (Janus kinase). The IL6/ STAT3 signal is often abnormally activated in inflammatory cancer cells and closely associated with tumorigenic phenotypes such as cell survival, growth and migration. In our previous study, HAUL (Human PAK4 Associated Ubiquitin Ligase) was identified as a functional oncogene and a key regulator of PAK4-associated TNF- $\alpha$ (Tumor Necrosis Factor $\alpha$ ) signaling, which is important in the cancer inflammation. Here, we present experimental evidence for another oncogenic function of HAUL, i.e., as a regulator of STAT3 signaling. We found that the IL6-induced activation of STAT3 signaling is enhanced or diminished in a HAUL-dependent manner. We also show that HAUL physically interacts with JAK and regulates IL6-induced JAK activation possibly by blocking the inhibitory function of SOCS3 (Suppressor of cytokine signaling-3) against JAK signaling. Furthermore, IL6-induced tumorigenic phenotypes such as cell survival, growth and migration were also regulated depending on the expression level of HAUL. Taken together, these results suggest that HAUL functions as a key regulator of IL-6/STAT3 signaling pathway and thus is a potential target for the treatment of inflammatory cancers.

\section{PE-082 \\ Metabolic Signature Genes Associated with Susceptibility to PKM2 Gene Ablation in Cancer Cells}

Yuri Jung ${ }^{1,3}$, Ye Jin Jang ${ }^{1}$, Min Ho Kang ${ }^{1}$, Young Soo Park ${ }^{1,3}$, Su Jin Oh ${ }^{1,3}$, Dong Chul Lee ${ }^{1}$, Zhi Xie ${ }^{2}$, Hyang-Sook Yoo ${ }^{7}$, Kyung Chan Park ${ }^{1 *}$, Young $/ l$ Yeom $^{1,3 *}$

${ }^{1}$ Medical Genomics Research Center, KRIBB, Daejeon, Republic of Korea; ${ }^{2}$ Pfizer Global Research and Development, Science Center Drive, San Diego, California, United States; ${ }^{3}$ Department of Functional Genomics, University of Science and Technology, Daejeon, Korea

Pyruvate kinase, muscle type 2 (PKM2), is a key factor in the aerobic glycolysis of cancer cells. In our experiments, liver cancer cell lines exhibited a range of sensitivity to PKM2 knockdown-mediated growth inhibition. We speculated that this differential sensitivity is attributable to the variable dependency on glycolysis for the growth of different cell lines. Transcriptome data revealed overexpression of a glucose transporter (GLUT3) and a lactate transporter (MCT4) genes in PKM2 knockdown-sensitive cells. PKM2 knockdownresistant cells expressed high levels of the lactate dehydrogenase B (LDHB) and glycine decarboxylase (GLDC) genes. Concordant with the gene expression results, PKM2 knockdown-sensitive cells generated high levels of lactate. In addition, ATP production was significantly reduced in the PKM2 knockdown-sensitive cells treated with a glucose analog, indicative of dependency of their cellular energetics on lactate-producing glycolysis. The PKM2 knockdownresistant cells were further subdivided into less glycolytic and more (glycolysis branch pathway-dependent) glycolytic groups. Our findings collectively support the utility of PKM2 as a therapeutic target for high lactate-producing glycolytic hepatocellular carcinoma (HCC). 


\section{PE-083 \\ Lipocalin-2 Negatively Modulates the EMT Change Through the EGF (TGF-beta1)/Lcn2/ Twist1 Pathway in Hepatocellular Carcinoma}

Yun-Peng Wang ${ }^{1}$, Goung-Ran Yu ${ }^{1}$, Mi-Jin Lee ${ }^{1}$, Sang-Yeop Lee ${ }^{2}$, In-Sun Chu' ${ }^{2}$, Sun-Hee Leem ${ }^{3}$, Dae-Ghon Kim ${ }^{7}$

${ }^{1}$ Division of Gastroenterology and Hepatology, the Research Institute of Clinical Medicine, Department of Internal Medicine, Chonbuk National University Medical School and Hospital, Jeonju, ${ }^{2}$ Korea Bioinformation Center, Korea Research Institute of Bioscience and Biotechnology, Daejeon, ${ }^{3}$ Department of Biological Science, Dong-A University, Busan, Korea

Background: Lipocalin-2 is preferentially expressed in hepatocellular carcinoma. However, the functional role of Lcn2 in HCC progression is still poorly understood, particularly with respect to its involvement in invasion and metastasis. The purpose of this study was to investigate whether Lcn2 is associated with the EMT change of HCC and to elucidate its signaling pathway.

Methods: Lcn2 mRNA expression was confirmed by RT-PCR analysis. Lcn2 protein expression was investigated by immunohistochemistry or Western blot analyses. The proliferation, migration, and invasion ability were measured by MTT, wound healing and invasion assays in HCC cells (SH-J1) stably expressing Lcn2. Tumor growing and metastasis by Lcn2 was tested in vivo animal model.

Results: Lcn2 is preferentially expressed in well differentiated HCC versus liver cirrhosis tissues, and its expression is positively correlated with the Edmondson differentiation grade of HCC. The characteristics of EMT are reversed by adenoviral transduction of Lcn2 into SH-J1 cells, including the down-regulation of N-cadherin, vimentin, a-SMA, and fibronectin, and the concomitant up-regulation of CK8, CK18, and desmoplakin I/II. Knock-down of Lcn2 by shRNA in HKK-2 cells highly expressing Lcn2 was associated with EMT change. EGF or TGF- $\beta 1$ treatment resulted in down-regulation of Lcn2, subsequently accompanied by Twist 1 expression and EMT change in HCC cells. Stable Lcn2 expression in HCC cells reduced Twist1 expression, led to inhibition of cell proliferation and invasion in vitro, and suppressed tumor growth and metastatic ability in a mouse model. Furthermore, EGF or TGF- $\beta 1$ treatment barely changed EMT marker expression in the SH-J1 cells ectopically expressing Lcn2. Ectopic expression of Twist1 induced EMT marker expression even in cells expressing Lcn2, indicating that Lcn2 functions downstream of growth factors and upstream of Twist1.

Conclusions: These findings suggest that Lcn 2 can negatively modulate the EMT change in HCC cells through the EGF (or TGF- $\beta 1$ )/Lcn2/Twist1 pathway. Thus, Lcn2 may be a candidate metastasis suppressor and a potential therapeutic target in HCC.

\author{
PE-084 \\ Establishment of Mouse Avatar Models \\ Generated From Patients with Hepatocellular \\ Carcinoma \\ Joong-Won Park ${ }^{1,2 *}$, Bo Hyun Kim ${ }^{7}$, Sung Hoon Kim ${ }^{1,2}$, \\ Jinsook Kim², Sook-Kyung Lee', Jung Ahn Lee', Eun \\ Kyung Hong ${ }^{1,2}$ \\ ${ }^{1}$ Center for Liver Cancer, ${ }^{2}$ Liver and Pancreatobiliary \\ Cancer Branch of Translational and Clinical Research, \\ National Cancer Center, Goyang, Korea
}

Background: Recurrence or progression of hepatocellular carcinoma (HCC) after curative treatments frequently occurs and succeeding treatments are necessary for control of disease. Systemic chemotherapy is one option of management for progression of HCC, but there are no reliable biomarkers to predict the tumor response of anti-cancer drugs including sorafenib. Mouse xenograft models, 'avatars' could allow physicians to find the most effective drugs before giving them to a patient. We planned to establish mouse avatar models generated from patients with HCC.

Methods: HCC samples were obtained from surgical resection of patients. Tumor samples were immediately minced into fine fragments and then mixed $1: 1(\mathrm{v} / \mathrm{v})$ with Matrigel. The tissue mixture was subcutaneously injected in both flanks of NOD/SCID mice. Growth of implanted tumor xenografts was monitored twice a week. For serial transplantation, tumor-bearing animals were sacrificed and acquired tumors were injected in successive BALB/c-nu mice. To investigate the effects of anti-cancer drugs on HCC xenografts mouse models were treated with drugs and monitored by measurement of tumor size.

Results: The first generation of xenograft mouse was established within 3-4 months after initial transplantation of patient HCC tumor. Serial transplantations and passages were done to establish sufficient numbers of xenograft mice for drug response tests. Serial passage of tumors has been done every 1-2 months. Improved success rate of xenograft models was achieved after learning period. Drug response experiments with sorafenib and other anti-cancer drugs are ongoing and will be presented.

Conclusions: Patient HCC xenograft avatar model is established and may be useful for screening anti-cancer drugs, especially in patients with high risk of recurrence after resection. 


\author{
PE-085 \\ JNK Signaling is Associated with Side \\ Population by Anti-Cancer Drugs in \\ Hepatocarcinoma Cells \\ Jong Bin Kim ${ }^{1}$, Hye Ri Kim', Seo-Young Park², \\ Yoon Jun, Kim ${ }^{1}$ \\ ${ }^{1}$ Department of Internal Medicine and Liver Research \\ Institute, Seoul National University College of Medicine, \\ Seoul, ${ }^{2}$ Clinical Research Institute, Seoul National \\ University Hospital, Seoul, Korea
}

Background: Liver cancer is one of the most drugresistant cancers, and cancer stem cells are related with drug resistance. JNK signaling are involved in drug resistance, and the side population can be use as a model to study cancer stem cells in liver cancer.

Methods: We sought to study the direct relationship among SP cells and c-jun-N-terminal kinase (JNK) signaling in hepatocarcinoma cells. Huh7 cells were treated with the anticancer drugs 5-fluorouracil (5-FU) and paclitaxel, and their proliferation and the SP fraction were examined. The expression of phospho-stress-activated protein kinase/JNK in treated cells was evaluated by immunoblotting.

Results: Both 5-FU and paclitaxel induced an increase in the number of SP cells, JNK phosphorylation, and decreased proliferation. Huh7 and HepG2 cells treated with SP600125 (JNK inhibitor) to study relationship among SP cells and c-jun$\mathrm{N}$-terminal kinase (JNK) signaling. The increase in the number of SP cells and the phosphorylation of JNK, c-Jun were blocked by SP600125 in these cells. Phosphorylation of JNK and c-Jun upregulated in cancer tissue.

Conclusions: In conclusion, these results indicate that the number of SP cells and phosphorylation of JNK increased after treatment with anti-cancer drugs and that this increase can be block by inhibiting JNK signaling. These findings suggest that drug resistance in the liver cancer might be involved in the increased number of SP cells after JNK activation.

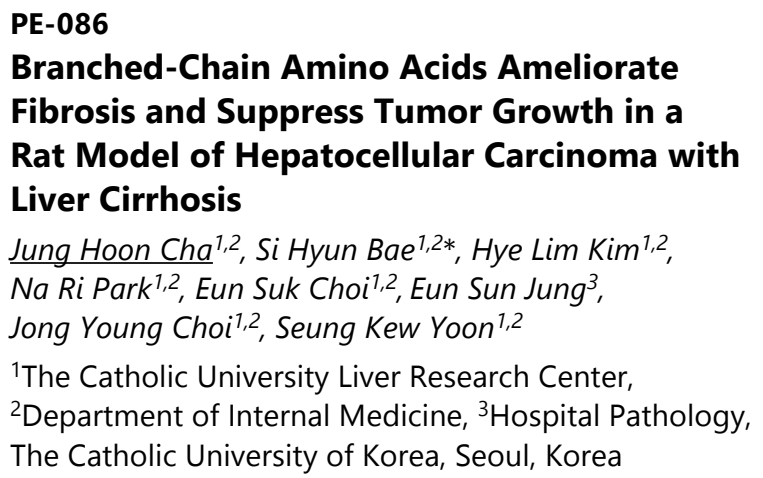

${ }^{1}$ The Catholic University Liver Research Center,

${ }^{2}$ Department of Internal Medicine, ${ }^{3}$ Hospital Pathology,

The Catholic University of Korea, Seoul, Korea

Background: Recent studies have revealed that branched-chain amino acids (BCAA) reduce the development of hepatocellular carcinoma (HCC) in patients with obesity and hepatitis $\mathrm{C}$ virus infection by improving insulin resistance (IR). The aim of this study was to examine the anti-cancer and anti-fibrotic effects of BCAA on the development of diethylnitrosamine (DEN)-induced HCC and liver cirrhosis in a rat model.

Methods: Male SD rats received weekly intraperitoneal injections of DEN (50 mg/kg of body weight) for 16 weeks to induce HCC. They were fed a diet containing $3 \%$ casein, $3 \%$ or $6 \%$ BCAA for 13 weeks beginning 6 weeks after DEN administration. For each case, 10 sections of every group were used to analyze HCC at 16 and 19 weeks of DEN administration. The effect of BCAA was evaluated in tumor tissues by histopathologic analyses, reverse transcription-polymerase chain reaction, and Western blotting.

Results: The mean area and number of dysplastic nodules (DNs) and tumors in the casein group tended to be larger than those in the BCAA group 16 weeks after DEN administration. The mean fibrotic area in the BCAA group was smaller than that in the casein group. The BCAA group showed decreased mRNA levels for markers of fibrosis, angiogenesis, and apoptosis inhibition. Compared with the casein group, the BCAA group had lower levels of $\alpha$-smooth muscle actin, vascular endothelial growth factor, $\mathrm{p}-\beta$-catenin, p-p38 mitogen-activated protein kinase, proliferating cell nuclear antigen, and caspase-3 protein expression, as well as a higher level of cleaved caspase-3 protein expression.

Conclusions: BCAA supplementation of the diet ameliorated liver fibrosis and HCC development in a DEN-induced rat model of HCC with liver cirrhosis, but not in the IR model. These results provide a rationale for anti-fibrosis and chemoprevention using BCAA treatment for HCC with liver cirrhosis, as well as decreasing the ammonia level. 


\section{PE-087 \\ Interleukin-8 is Associated with Liver Cancer Progression}

Seo-Young Park ${ }^{1,2}$, Jiyou Han², Jong Bin Kim³, Yoon-Jun Kim ${ }^{3}$, Man Gil Yang ${ }^{1}$, Jong-Hoon Kim²

${ }^{1}$ Biomedical Institute, Seoul National University Hospital, Seoul, ${ }^{2}$ Laboratory of Stem Cell Biology, Division of Biotechnology, College of Life Sciences and Biotechnology, Korea University, Seoul, ${ }^{3}$ Liver Research Institute, Seoul National University College of Medicine, Seoul, Korea

Background: Interleukin-8 (IL-8) has been suggested as a prognostic biomarker for human hepatic carcinoma (HCC), but its roles in the HCC progression and drug resistance has not been studied. In the present study, we investigated the role and underlying mechanism of IL- 8 in the progressive growth of HCC using two different human hepatoma cell lines, HepG and Huh7

Methods: The expression of IL-8 and side population were examined to identify their correlation with chemoresistance and progressive growth in HCC cell lines. The potential mechanisms of IL-8 associated with the ATP-binding cassette transporters were determined by immunostaining in specimens from HCC patients and xenograft models. Tumorigenicity of hepatoma cells was evaluated in vivo after silencing IL-8 gene.

Results: Here we demonstrate that treatment of hepatoma cells with anti-cancer drugs, paclitaxel and doxorubicin, increased the production of IL- 8 and its receptor, IL-8RA, and proportion of SP cells. In addition, recombinant IL-8 increased SP fraction and the expression of multidrug resistance 1 , one of ATP-binding cassette (ABC) transporters and decreased the sensitivity to the anticancer treatment. Silencing of IL-8 gene using small interfering RNA decreased both the ratio of SP cells and drug resistance properties. We also show that both IL-8 and ABC transporters are highly expressed in xenograft HCC tissues and clinical HCC samples and show that knockdown of IL-8 in hepatoma cells significantly reduce tumor size in vivo.

Conclusions: These data suggest that IL-8 plays a critical role in HCC progression and chemo-resistant properties and may be a potential therapeutic target in the treatment of HCC.

\author{
PE-088 \\ Livin is Associated with Invasive and \\ Oncogenic Phenotypes of Human \\ Hepatocellular Carcinoma Cells \\ Sung-Bum Cho ${ }^{1}$, Young-Lan Park ${ }^{1}$, Nuri Kim ${ }^{1}$, \\ Cho-Yun Chung ${ }^{1}$, Hyung-Chul Park ${ }^{1}$, Jong-Sun Kim ${ }^{1}$, \\ Dae-Seong Myung ${ }^{1}$, Kyung-Hwa Lee ${ }^{2}$, Jae-Hyuk Lee ${ }^{2}$, \\ Sung-Kyu Choi ${ }^{7}$, Young-Eun Joo ${ }^{1}$ \\ Departments of ${ }^{1}$ Internal Medicine and ${ }^{2}$ Pathology, \\ Chonnam National University Medical School, Gwangju, \\ Korea
}

Background: Livin, a member of the inhibitors of apoptosis proteins, has been found to be expressed in variable cancers, where its expression is considered to be a poor prognostic marker. The purposes of this study were to observe the effect of Livin on tumor cell behaviors of human hepatocellular carcinoma (HCC) cells and to evaluate its expression in human HCC tissues and its relation to prognosis.

Methods: We investigated the biologic role of Livin on tumor cell behaviors by using the small interfering RNA (siRNA) in human HCC cell line, HepG2. The migration, invasion and proliferation assays were performed. To evaluate the impact of Livin on apoptosis and cell cycle, we performed flow cytometric analyses and Western blotting. The expression of Livin by western blotting and immunohistochemistry was investigated in human HCC tissues.

Results: Knockdown of Livin suppressed tumor cell migration, invasion and proliferation in human HCC cells. The proportion of apoptotic cells induced by knockdown of Livin was greater than that induced by the scramble siRNA-transfected cells in human HCC cells. Knockdown of Livin activated cleaved caspase-3, -7, -9 and PARP, leading to induction of apoptosis in human HCC cells. Knockdown of Livin induced the G2/M phase arrest by decreasing cyclin D1, CDK4 and CDK6, and by increasing p16, p21, p27 and p57 expression in human HCC cells. The expression of Livin was significantly elevated in human HCC tissues compared to normal hepatic tissues. But Livin expression is not associated with various clinicopathological parameters including survival.

Conclusions: These results suggest that Livin is associated with the alteration of invasive and oncogenic phenotypes such as tumor cell migration, invasion, proliferation, resistance to apoptosis and cell cycle arrest in human HCC cells. 


\section{PE-089 \\ Extensive Apoptosis of Hepatocellular Carcinoma Under Hypoxia \\ Sung Hoon Choi, Seung Up Kim, Do Young Kim, Weon Sang Ro, Sang Hoon Ahn, Seungtaek Kim, Kwang-Hyub Han and Jun Yong Park \\ Yonsei University College of Medicine, Seoul, Korea}

Background: Hypoxia-inducible factor- $1 \alpha(\mathrm{HIF}-1 \alpha)$ is a critical transcription factor involved in multiple aspects of oncogenesis, including proliferation, survival under hypoxia. Interleukin 8 (IL-8) also induces survival of endothelial cells under hypoxic conditions. This study aims to evaluate whether adenovirus-mediated small hairpin RNAs for HIF- $1 \alpha$ (shHIF-1 $\alpha$ ) and IL-8 (shIL-8) induced apoptosis of the hepatocellular carcinoma (HCC) and endothelial cell lines.

Methods: HCC cell lines were infected with adenovirus expressing shRNA for HIF- $1 \alpha$ and IL-8 and maintained under hypoxic conditions $\left(1 \% \mathrm{O}_{2}, 24\right.$ hours $)$. The expression levels of HIF- $1 \alpha$ and apoptotic and growth factors were examined by real-time quantitative RT-PCR and immunoblot. We also investigated apoptosis by TUNEL assay and measured concentration of cytochrome $\mathrm{C}$.

Results: Inhibition of HIF- $1 \alpha$ and IL- 8 up-regulated the expression of apoptotic factors while down-regulating antiapoptotic factors simultaneously. Knockdown of HIF-1 $\alpha$ and IL-8 increased concentration of cytochrome $\mathrm{C}$ and enhanced DNA fragmentation in HCC cell lines and HUVECs. Moreover, culture supernatant collected from the knockdown of HIF- $1 \alpha$ and IL-8 in HCC cell lines induced apoptosis of HUVECs under hypoxia.

Conclusions: These data suggest that adenovirusmediated knockdown of HIF-1 $\alpha$ and IL-8 induced apoptosis and affected tumor environment.

\section{PE-090}

Phase 2b Randomized Trial of Pexa-Vec (pexastimogene devacirepvec; JX-594), a Targeted Oncolytic Vaccinia Virus, Plus Best Supportive Care (BSC) Versus BSC Alone in Patients with Advanced Hepatocellular Carcinoma who Have Failed Sorafenib Treatment (TRAVERSE)

Jeong Heo, Han Chu Lee, Seung Woon Paik, Won Young Tak, Jung-Hwan Yoon, Kwan Soo Byun, Kwang-Hyub Han, Hyung Joon Yim, Mong Cho, Caroline J. Breitbach, Rick Patt, Riccardo Lencioni, Michel Homerin, Jean-Marc Limacher, Theresa Hickman, Lara Longpre, James Burke, Davi

Pusan National University Hospital, Pusan, Korea

Background: Pexa-Vec is a targeted oncolytic and immunotherapeutic vaccinia virus engineered to express human granulocyte-macrophage colony stimulating factor (GM-CSF). Direct oncolysis plus GM-CSF expression stimulates tumor vascular disruption and anti-tumor immunity (Nature Rev Cancer 2009). Pexa-Vec was well-tolerated in Phase 1 trials and was shown to replicate in metastatic tumors following intratumoral (IT) or intravenous (IV) administration (Lancet Oncol 2008 and Nature 2011). A randomized high vs low dose Phase 2 trial in 30 patients with advanced HCC, demonstrated prolonged survival in the high-dose Pexa-Vec arm (median survival 14.1 mo vs 6.7 mo; Hazard Ratio 0.39, p = 0.02) (AASLD Annual Meeting, 2011, LB1).

Methods: TRAVERSE is a Phase $2 \mathrm{~b}$ randomized, openlabel, multi-center trial in patients with advanced HCC who have failed sorafenib treatment. Approximately 120 patients will be randomized 2:1 to Pexa-Vec plus BSC versus BSC, respectively. Randomization will be stratified by region (Asian vs non-Asian); sorafenib intolerant vs refractory; and presence vs absence of extra-hepatic disease. The primary objective is to determine overall survival. Main inclusion criteria are advanced HCC having failed sorafenib (intolerance or radiographic progression during or $<3$ months following last sorafenib), Child-Pugh A-B7 (no ascites), acceptable hematologic function. Assuming a median overall survival of 4.0 months with BSC and a target hazard ratio of 0.57 (corresponding to an experimental arm median survival of 7.0 months), 73 events (deaths) will provide $70 \%$ power at 1-sided alpha $=0.05$ to detect a difference in overall survival between the treatment groups using a stratified logrank test. Patients randomized to Pexa-Vec will receive a dose of 109 plaque forming units (pfu) IV on Day 1 followed by five IT treatments between Day 8 and Week 18. Enrollment has begun on this study with clinical trial registry number of NCT01387555. 


\section{PE-091 \\ Characteristics of Combined Hepatocelluar- Cholangiocarcinoma and Comparison with Intrahepatic Cholangiocarcinoma

\author{
Sung Hoon Kim ${ }^{7}$, Gi Hong Choi ${ }^{2}$, Jun Sub Choi ${ }^{2}$, \\ Kyung Sik Kim² \\ ${ }^{1}$ Department of Surgery, Yonsei University Wonju \\ College of Medicine, ${ }^{2}$ Department of Surgery, Yonsei \\ University College of Medicine, Seoul, Korea
}

Background: The 7th American Joint Committee on Cancer (AJCC) currently classifies combined hepatocellularcholangiocarcinoma (cHCC-CC) and intrahepatic cholangiocarcinoma (ICC) into one category. Study outcomes comparing the two carcinomas have shown contrary results. This study was designed to compare the survival and prognostic factors of both carcinomas.

Methods: We retrospectively reviewed the medical records of 107 patients with CHCC-CC or ICC who underwent liver resection between January 2000 and December 2009.

Results: Thirty patients (28\%) were diagnosed with cHCC-CC, and 77 patients (72\%) had ICC. Disease-free survival (DFS) was poorer in the cHCC-CC patients (six months), and the overall survival (OS) durations were similar ( $p=0.477)$ between cHCC-CC (58 months) and ICC (45 months) patients. A tumor size larger than $5 \mathrm{~cm}$, vascular invasion and lymph node (LN) metastasis were prognostic factors in all patients. However, tumor size and LN metastasis in cHCC-CC patients and carbohydrate antigen 19-9, differentiation and LN metastasis in ICC patients were found to be independent prognostic factors.

Conclusions: Patients with cHCC-CC showed poorer DFS and similar OS rates compared to those with ICC. Our study revealed different prognostic factors in cHCC-CC. To understand more accurately cHCC-CC's prognosis, difference of genetic characteristics and tumor biology should be further evaluated.

\section{PE-092 \\ Risk Factors for Diabetes in Patients with Chronic Liver Disease, Using Transient Elastography}

Jem Ma Ahn, Ju Yeon Cho, Won Sohn, Yong-Han Paik, Ho Young Lim, Yang Won Min, Geum-Youn Gwak, Moon Seok Choi, Joon Hyeok Lee, Kwang Cheol Koh, Seung Woon Paik, Byung Chul Yoo

Department of Medicine, Samsung Medical Center, Sungkyunkwan University School of Medicine, Seoul, Korea

Background: Our aim was to evaluate whether there was relationship of etiology, various life styles, hepatic fibrosis, and steatosis with prevalence of diabetes in chronic liver disease, using transient elastography.
Methods: The study population consisted of 567 chronic liver disease patients. Diabetes was diagnosed when it was previously diagnosed by doctor, or fasting plasma glucose was more than $126 \mathrm{mg} / \mathrm{dL}$ at least 2 times. Fibrosis and steatosis was measured by transient elastography. Significant fibrosis (F2/3) was defined as more than $8 \mathrm{kPa}$ in LSM, and Severe fibrosis (F4) as more than $14 \mathrm{kPa}$ in LSM. The degree of steatosis was graded by CAP score (mild steatosis (S1): 239 258db/m, moderate steatosis (S2): 259 292db/m, severe steatosis (S3): >292 db/m). Respecting life styles, Age (cut-off: 50 years old), gender, obesity (cut-off: BMI 25), smoking, hypertension, hyperlipidemia, alcohol consumption, and exercise were used.

Results: 84 of 567 were with diabetes (15\%), and 184 of 567 were with impaired fasting glucose $(32 \%)$. According to the etiology, alcohol (35\%), NAFLD (22\%), HCV (17\%) showed high prevalence of diabetes compared with HBV $(12 \%)(\mathrm{P}<$ $0.001)$. Impaired fasting glucose showed similarly high prevalence of diabetes in all etiology (30 37\%, P < 0.001). Diabetes was observed more commonly with the higher degree of fibrosis (F0/1(9\%), F2/3(14\%), F4(31\%), P < 0.001)). Prevalence of diabetes was higher in the group of moderate and severe steatosis (S0/1(13\%), S2/3(18\%), P < 0.001). About life styles, there was significant association of diabetes with older age ( $>50$ years), gender (male), obesity (BMI $>25$ ), smoking, hypertension, hyperlipidemia.

Conclusions: Our study suggests that prevalence of diabetes is high in chronic liver disease except HBV. About life style, older age, male, obesity, smoking, hypertension, and hyperlipidemia are shown as risk factors of diabetes in chronic liver disease. Moreover our findings indicate that the degree of fibrosis, and steatosis, measured by transient elastography, have significant relationship with prevalence of diabetes in chronic liver disease.

\section{PE-093 \\ Comparison of Lamivudine Plus Adefovir Therapy versus Entecavir with or without Adefovir Therapy for Adefovir-Resistant Chronic Hepatitis B \\ Seong Hee Kang Hyung Joon Yim, Hae Rim Kim, Keunhee Kang, Sang Jun Suh, Hyun Jung Lee, Eileen L. Yoon, Ji Hoon Kim, Yeon Seok Seo, Jong Eun Yeon, Kwan Soo Byun \\ Department of Internal Medicine, Korea University Medical College, Seoul, Korea}

Background: Data regarding the management of adefovir (ADV) resistance are still limited. The aim of this study is to investigate treatment outcomes of rescue therapy in ADVresistant chronic hepatitis $\mathrm{B}$ (CHB) patients.

Methods: Adult CHB patients who began rescue therapy due to documented genotypic resistance mutations to ADV 
between October 2006 and July 2012 were retrospectively reviewed.

Results: Fifty-eight patients were included in this study. Most patients had history of lamivudine (LAM) resistance. Treatment response was evaluated at 3-month intervals up to 12 months. The cumulative rate of complete virologic response (CVR) in hepatitis B virus (HBV) infected patients (HBV DNA $<60 \mathrm{IU} / \mathrm{mL}$ ) was $17.2 \%, 27.8 \%, 29.3 \%$, and $29.3 \%$ following $3,6,9$, and 12 months of rescue therapy. Thirty-four patients were treated with a combination of LAM plus ADV (LAM+ADV group) and 24 patients were treated with entecavir (ETV)based therapy (ETV with or without ADV therapy, ETV \pm ADV group). The cumulative CVR rate was significantly higher in the ETV \pm ADV group than in the LAM+ADV group at month 12 ( $45.8 \%$ vs. $17.8 \%$, respectively, $p=0.031$ ) Multivariate analysis showed that only pre-treatment serum HBV DNA levels at $<6 \log 10 \mathrm{IU} / \mathrm{mL}$ was associated with CVR (hazard ratio: $7.175, \mathrm{p}=0.004$ ).

Conclusions: ETV \pm ADV therapy resulted in a significantly higher CVR rate in ADV-resistant patients, whereas baseline HBV DNA level was the most important predictive factor for CVR. This study suggests need of early switching from ADV to a rescue therapy such as ETV \pm ADV at the time of low-level viremia.

\section{PE-094 \\ Quantitative Hepatitis B Surface Antigen Kinetics in Entecavir Treated Patients}

Ju-Yeon Cho ${ }^{1}$, Yong-Han Paik ${ }^{1 *}$, Won Sohn ${ }^{1}$, Seon Woo Kim², Sook Young Woo' ${ }^{2}$, Geum-Youn Gwak', Moon Seok Choi ${ }^{1}$, Joon Hyeok Lee ${ }^{1}$, Kwang Cheol Koh', Seung Woon Paik ${ }^{1}$, Byung Chul Yoo ${ }^{1}$

${ }^{1}$ Department of Medicine, ${ }^{2}$ Department of Statistics, Samsung Medical Center, Sungkyunkwan University School of Medicine, Seoul, Korea

Background: Hepatitis B surface antigen (HBsAg) loss is associated with immunological control of the hepatitis $B$ virus and durable suppression of viral replication. HBsAg levels reflect transcription of closed covalent circular DNA in patients with chronic hepatitis B (CHB). The aim of this study was to investigate the on-treatment kinetics of quantitative HBsAg during entecavir therapy to predict the treatment period needed to achieve HBsAg seroconversion.

Methods: From a cohort of 1006 CHB treatment-naïve patients who were started on entecavir, 425 patients with a quantitative HBsAg value after initiation of entecavir were selected. Among the patients, 321 patients $(75.1 \%)$ had more than 2 serial samples. The kinetics of quantitative HBsAg decline was assessed using 1465 samples from 413 patients with normal distribution and homoscedasticity with mixed linear model to predict the time to clear HBsAg while on entecavir treatment.
Results: Among the 413 patients, 213 patients (51.6\%) were $\mathrm{HBeAg}$ positive and 200 patients (48.4\%) were $\mathrm{HBeAg}$ negative. At baseline, the age of the $\mathrm{HBeAg}(-)$ group was significantly older $(\mathrm{p}<0.001)$ and the level of HBV-DNA was significantly lower $(\mathrm{p}<0.001)$ compared to the HBeAg $(+)$ group. During a median follow up of 49.5 months, the quantitative HBsAg level showed a slow but consistent decrease in value regardless of the HBeAg status. The HBeAg-positive group had a mean slope of $-0.0036 \pm 0.0003 \log _{10} \mathrm{IU} /$ month $(\mathrm{p}<0.001)$ and the HBeAg-negative group had a mean slope of $-0.0037 \pm$ $0.0004 \log _{10} \mathrm{IU} /$ month $(\mathrm{p}<0.001)$. The calculated time to clear quantitative HBsAg in HBeAg-positive and HBeAgnegative groups were 73 years and 71 years, respectively.

Conclusions: The analysis of kinetics for HBsAg level during entecavir therapy suggests the treatment period required to achieve quantitative HBsAg clearance during entecavir therapy is life-long, regardless of the HBeAg status of chronic hepatitis B patients.

\section{PE-095 Assessment of Liver Fibrosis by Real Time
Sonoelastography \\ Young Woon Kim ${ }^{1}$, Soon Woo Nam ${ }^{1}$, Jung Hyun Kwon ${ }^{1}$, Jeong Won Jang ${ }^{2}$, Min Ju Kim ${ }^{1}$, Byong Sun Oh', Kyu Won Chung ${ }^{1}$, Eunsu Park ${ }^{3}$ \\ ${ }^{1}$ Department of Internal Medicine, The Catholic University of Korea, Incheon St. Mary's Hospital, Incheon, 2Department of Internal Medicine, The Catholic University of Korea, Seoul St. Mary's Hospital, Seoul, ${ }^{3}$ Department of pathology, Catholic University of Korea, Incheon St. Mary's Hospital, Incheon, Korea}

Background: Recently, real-time transient elastography has been introduced for noninvasive staging of liver fibrosis, which can be performed with conventional ultrasound probes during a routine sonography examination. In contrast FibroScan, there are few reports about the real time transient elastography. The aim of the study was to investigate the diagnostic usefulness of real-time elastography for hepatic fibrosis

Methods: Eighty-five consecutive patients were prospectively enrolled, who underwent both liver biopsy and realtime elastography for chronic viral hepatitis from March 2011 to March 2013 in Incheon St. Mary's Hospital. Real-time elastography (Hitach avius and EUP-L52 probe, Tokyo, Japan) was performed by one examiner and ultrasonography guided liver biopsy was done at the same time. The degree of liver fibrosis was confirmed according to the Metavir scoring system by one pathologist. In addition, aspartate transaminase-to-platelet ratio index (APRI) was measured

Results: The mean age of the patients was $45.9 \pm 10.3$ years, and 54 patients were male. The patients consisted of chronic hepatitis B $(52,61.2 \%)$, chronic hepaitits C (33, $38.8 \%)$. The fibrosis stage ranged in 14 patient $(16.3 \%)$ for $\mathrm{F} 0$, 
27 patients (31.4\%) for $\mathrm{F} 1,27$ patients (31.4\%) for $\mathrm{F} 3,17$ patients $(19.8 \%)$ for $\mathrm{F} 4$. The mean real-time elastography value significantly increased according to the fibrosis; $3.14 \pm$ 0.62 for F0, $3.28 \pm 0.42$ for F1, $3.43 \pm 0.53$ for F3 and $4.09 \pm$ 1.03 for $F 4(p=0.000)$. The real-time elastography value, APRI, the levels of platelet, albumin and prothrombin time INR were different between the patients with the advanced fibrosis $(\geq \mathrm{F} 3)$ and those with the mild fibrosis $(\leq \mathrm{F} 1)$. For predicting the advanced fibrosis $(\geq \mathrm{F} 3)$ in multivariate analysis, the real-time elastography value and prothrombin time INR were significant factors.

Conclusions: The real-time elastography is a new and promising sonography-based noninvasive method for the assessment of liver fibrosis in patients with chronic viral hepatitis.

\section{PE-096 \\ Viral Evolutionary Changes during Tenofovir Treatment in a Chronic Hepatitis B Patient with Sequential Nucleos(t)ide Therapy}

Hyun Woong Lee, Sang Wook Kim, Tae Young Park, Sung Jin Park, Hyung Joon Kim*

Department of Internal Medicine, Chung-Ang

University College of Medicine, Seoul, Korea

Background: Although the rtA194T hepatitis B virus (HBV) polymerase mutation recently identified in tenofovir (TDF)-treated human immunodeficiency virus (HIV)/HBVcoinfected patients, this mutation did not confer in vitro resistance to TDF as a single mutation or in lamivudine (LAM)resistant viral background. We experienced one case of virological and biochemical breakthrough during TDF treatment of a chronic hepatitis $\mathrm{B}$ ( $\mathrm{CHB}$ ) patient who received sequential antiviral therapy.

Case: A 54-year-old man diagnosed with hepatitis B virus e antigen-positive CHB had received entecavir (ETV) $1 \mathrm{mg} /$ day treatment for 120 weeks following an initial unsuccessful LAM treatment (rtL180M, rtM204V/I). Sequentially, virological and biochemical breakthrough with ETV mutation (rtT184T/L) were developed. The therapy had been switched to LAM and adefovir (ADV) for 24 weeks. This led only to a temporary HBV DNA decline, which soon was followed by virological and biochemical breakthrough despite the lack of known ADV resistance mutations. The therapy had been switched to TDF monotherapy for 1 year. However, the levels of HBV DNA and alanine transaminase rebounded from 3.8 $\log _{10}$ copies/mL and $23 \mathrm{IU} / \mathrm{mL}$, respectively, at weeks 24 to 7.0 $\log _{10}$ copies $/ \mathrm{mL}$ and $357 \mathrm{IU} / \mathrm{m}$, respectively, at week 48 . A longitudinal clonal analysis of HBV genomes was performed by evaluating 50 clones from five different serum samples. This patient developed a high degree of resistance to TDF caused by a HBV strain bearing rtL180M, rtM204V/I, rtA200V, rtF221Y, rtS223A, rtV84M, rtI91F, rtR153Q, rtT184A/S,
rtV191I combined mutations without previous reported rtA194T mutation.

Conclusions: TDF resistance may emerge due to multisite polymerase mutations rather than single-site polymerase mutation. The persistence of complex mutants across the entire HBV genome might play a important role for the development of multi-drug resistance after failure of nucleos(t)ide analogues.

\section{PE-097 \\ Long Term Efficacy of Adefovir and Lamivudine Combination Therapy for Lamivudine Resistance: 5 Year Follow-Up Data}

Hyung Joon Yim ${ }^{1}$, Hae Rim Kim ${ }^{1}$, Sang Jun Suh ${ }^{7}$, Yeon Seok Seo ${ }^{2}$, Chang Wook Kim ${ }^{3}$, Chang Don Lee ${ }^{3}$, Sang Hoon Park', Myung Seok Lee ${ }^{4}$, Choong Kee Park ${ }^{5}$, Hee Bok Chae ${ }^{6}$, Moon Young Kim ${ }^{7}$, Soon Koo Baik', Yun Soo Kim ${ }^{8}$, Ju Hyun Kim ${ }^{8}$, Jung Il Lee ${ }^{9}$, Jin Woo Lee ${ }^{9}$, Sun Pyo Hong ${ }^{10}$, Soon Ho Um ${ }^{2}$

Department of Internal Medicine, ${ }^{1}$ Korea University Ansan Hospital, Ansan, ${ }^{2}$ Korea University Anam Hospital, Seoul, ${ }^{3}$ The Catholic University of Korea Uijeongbu St. Mary's Hospital, Uijeongbu, ${ }^{4} \mathrm{Hallym}$ University Kangnam Sacred Heart Hospital, Seoul, ${ }^{5}$ Hallym University Sacred Heart Hospital, Anyang, ${ }^{6}$ Chungbuk National University Hospital, Cheongju, ${ }^{7}$ Yonsei University Wonju Christian Hospital, Wonju,

${ }^{8}$ Gachon University Gil Hospital, Incheon, ${ }^{9}$ Inha University Hospital, Incheon, ${ }^{10}$ GeneMarix Inc., Yongin, Korea

Background: Lamivudine (LMV) resistance is still a challenging issue as it predisposes a multidrug resistance in the management of chronic hepatitis B (CHB). Combination of LMV and adefovir (ADV) has been suggested as a standard treatment for LMV resistance in the countries where tenofovir is not available. However long term efficacy of $A D V+L M V$ is needed to be further evaluated especially in genotype $C$ patients. The previous prospective study compared the efficacy of ADV+LMV combination with entecavir monotherapy for LMV resistant CHB after 2 years of treatment, and then patients were further followed-up up to 5 years. As ADV+LMV therapy was shown to be better than ETV monotherapy in the previous study, this current study was planned to evaluate the long-term efficacy of ADV+LMV therapy.

Methods: One hundred and ten CHB patients who had developed LMV resistance received ADV+LMV therapy up to 5 years. Virologic response (VR) rate which is defined as HBV DNA $<20 \mathrm{IU} / \mathrm{mL}$ was evaluated as a primary end point.

Result: The mean age was $44 \pm 12$ years and $77 \%$ were male. The proportion of HBeAg-positive patient was $79 \%$ $(87 / 110)$. All the patients had genotype C HBV. The mean 
serum HBV DNA levels were $7.05 \pm 1.07,2.57 \pm 1.54,2.20 \pm$ $1.25,2.27 \pm 1.05$ and $2.09 \pm 0.93 \log 10 \mathrm{IU} / \mathrm{ml}$ at baseline, month 12, 24, 36 and 48, respectively. The mean reductions in serum HBV DNA levels from baseline to $12,24,36$ and 48 months were $-4.68 \pm 1.58,-4.93 \pm 1.28,-4.92 \pm 1.33$ and $-5.06 \pm 1.06 \log 10 \mathrm{IU} / \mathrm{ml}$, respectively. The cumulative rate of VR was 53\% (58/110), virologic breakthrough 10\% (11/110) and genotypic resistance to adefovir 10\% (11/110) during median 36 (12-60) months. The rate of virologic breakthrough $(10.3 \%$ vs. $8.7 \%, \mathrm{P}=0.661)$ and genotypic resistance to $\mathrm{ADV}(11.6 \%$ vs. $4.8 \%, \mathrm{P}=0.688)$ was not different between HBeAg-positive and negative patients. However, the VR rate was higher in HBeAg-negative patients (82.6\% vs. $44.8 \%, \mathrm{P}=$ 0.002).

Conclusion: Long term ADV+LMV combination therapy lead to VR in a significant number of LMV resistant CHB patients with genotype C. However the efficacy was not satisfactory in HBeAg positive patients. Alternative therapy is certainly needed to be evaluated in this difficult-to-treat group.

\section{PE-098 \\ Can HBsAg Titer of Early Treatment Period Predict Virologic Response in Treatment Naïve CHB Patients during Entecavir Treatment?}

Hee Seung Lee, Jong Soon Jang, Seung Ho Lee, Myung Ho Youn, Hee Bok Chae

Department of Internal Medicine, Chungbuk National University Hospital, Cheongju, Korea

Background: The clinical usefulness of serum hepatitis B surface antigen (HBsAg) titer is not fully established yet. We aimed to determine whether the HBsAg titer can predict virologic response and HBeAg seroconversion during entecavir therapy or not.

Methods: Twenty-six treatment naïve chronic hepatitis $B$ patients receiving entecavir were enrolled. Serial viral markers such as quantitative HBsAg titer, HBV DNA level and qualitative $\mathrm{HBeAg}$, anti-HBe at baseline, 1, 3, 6, 9, 12 months after treatment. Serum HBsAg levels were measured with Architect HBsAg Assay (Abbott).

Results: (1) At baseline, mean age, $49.8 \pm 11.8$; female, 13(50\%), HBeAg positivity, 14(54\%), AST, $77.9 \pm 61.7 \mathrm{U} / \mathrm{L}$, ALT, $98.6 \pm 113.8$, total bilirubin $0.88 \pm 0.32 \mathrm{mg} / \mathrm{dl}$. The $\log _{10} \mathrm{HBsAg}$ titer copies/ml at baseline, 1, 3, 6, 9 and 12 month treatment were 3.37, 3.37, 3.44, 3.46, 3.44 and 3.43. (2) The baseline, 1month, 3month, and 6month HBsAg titer after commencing treatment were correlated with 12 month HBV DNA level, with p value $0.012,0.014,0.015$, and 0.035 , respectively by linear logistic regression. But, these HBsAg titers could not predict the undectability of HBV DNA at 12 month treatment by binary logistic regression. (3) The baseline, 1month, 3month, and 6month HBsAg titer after commencing treatment could not predict HBeAg loss or seroconversion in HBeAg positive patients. (4) The baseline, 1month, and 3month HBV DNA level after commencing treatment were associated with 12month HBV DNA level.

Conclusions: The HBsAg titers of early treatment period were correlated with HBV DNA level of 12month treatment, but they could not predict undectability of HBV DNA at 12 month treatment or HBeAg seroconversion. Further study with large number is needed.

\section{PE-099 \\ A Relationship between HCV RNA Viral Load and Clinical Outcome Inpatients with Hepatocellular Carcinoma}

Byoung Moo Lee ${ }^{7}$, Jae Young Jang ${ }^{1 *}$, Soung Won Jeong ${ }^{7}$, Woong Chuel Lee ${ }^{7}$, Eui Ju Park', Jin Nyoung Kim ${ }^{7}$, Sung Woo Ryu', Ji Hye Kim?', Sae Hwan Lee?, Sang Gyune Kim?', Sang-Woo Cha', Young Seok Kim ${ }^{1}$, Young Deok Cho', Hong Soo Kim ${ }^{7}$, Boo Sung Kim ${ }^{7}$

Institution for Digestive Research, Digestive Disease Center, 'Department of Internal Medicine, Soonchunhyang University Hospital, Seoul, Korea

Background/Aim: The HCV RNA viral load is the predictive factor for sustained viral response in the treatment of chronic hepatitis C. However, it has been reported that HCV RNA viral load is not predictive of eventual outcome of chronic hepatitis C. The relation between HCV RNA viral load and clinical outcome of hepatocellular carcinoma (HCC) is not elucidated, yet. We investigated the relation of HCV RNA viral load to the clinical outcome in HCC.

Methods: Among 453 HCC patients, 34 consecutive patients diagnosed with chronic hepatitis $C$ virus infection were classified into two groups by HCV RNA viral load. Group I ( $n=19)$ was defined as low HCV RNA viral load less than $2 \times 10^{6}$ copies/ml, and Group II $(n=15)$ defined as high HCV RNA viral load over $2 \times 10^{6}$ copies/ml. Characteristics of HCC, complications of cirrhosis, and overall survival were compared between the two groups.

Results: Sixteen patients were male (47.1\%) and the median age was 69 years. There was no significant difference in age, gender, Child-Pugh score, MELD score, tumor stage, portal vein invasion, distant metastasis, $\alpha$-fetoprotein, and histology between the two groups. For complications of cirrhosis including variceal hemorrhage, ascites, hepatorenal syndrome and hepatic encephalopathy during follow up, there was no significant difference between both groups. Median overall survival was 48.7 months (95\% CI, 30.9-66.6) for group I and 54.0 months ( $95 \%$ CI, 36.1-71.9) for group II. ( $p=$ 0.859).

Conclusion: HCV RNA viral load did not affect the clinical outcome in patients with hepatocellular carcinoma. 


\section{PE-100 \\ Diagnostic Performance of C-Reactive Protein and Neutrophil-to-Lymphocyte Ratio for Infection in Patients with Decompensated Cirrhosis}

Jung Hyun Kwon 1,2, Jeong Won Jang 1,3, Young Woon Kim $^{1,2}$, Byong Sun Oh' ${ }^{1,2}$, Soon Woo Nam ${ }^{1,2}$, Kyu Won Chung ${ }^{1,2}$, Dong Wook Jekarl ${ }^{4}$, Seungok Lee ${ }^{4}$

${ }^{1}$ Catholic Liver Research Center, ${ }^{2}$ Department of Internal Medicine, The Catholic University of Korea, Incheon St. Mary's Hospital, Incheon, ${ }^{3}$ Department of Internal Medicine, The Catholic University of Korea, Seoul St. Mary's Hospital, Seoul, ${ }^{4}$ Department of Laboratory Medicine, The Catholic University of Korea, Incheon St. Mary's Hospital, Inceon, Korea

Background: Infection is associated with poor prognosis, but often difficult to diagnose in cirrhotic patients. The role of clinical parameters such as systemic inflammatory response syndrome (SIRS) criteria in diagnosing infection remains unclear in cirrhotic population. The aim of this study was to evaluate the usefulness of inflammatory markers including C-reactive protein (CRP) and neutrophil-tolymphocyte ratio (NLR) for the diagnosis of infection in decompensated cirrhotic patients.

Methods: The study population consisted of 263 consecutive events in a cohort of 184 decompensated cirrhotic patients admitted to a tertiary centre from September 2011 to September 2012. The presence of SIRS and overt infection were evaluated. CRP and NLR were measured.

Results: The main cause of admission was uncontrolled ascites $(34.8 \%)$, followed by varix bleeding $(23.9 \%)$, and hepatic encephalopathy (13.6\%). Thirty six patients $(19.6 \%)$ had overt infection and 14 of $36(38.9 \%)$ patients only met the SIRS criteria. For the diagnosis of infection, baseline CRP $(\geq 12$ $\mathrm{mg} / \mathrm{L}$ ) and NLR (>4.4) were the significant factors compared to the Child-Pugh and MELD scores. Particularly in patients of the Child-Pugh C group, combined baseline CRP and NLR enhanced diagnostic accuracy of infection. Nine out of 36 patients developed delayed infection after admission and increased CRP from baseline to day 3 predicted the diagnosis of delayed infection. However, there were no differences in the presence of SIRS, WBC counts, Child-Pugh and MELD scores at baseline between the patients with or without infection.

Conclusions: The present study suggests that NLR as well as CRP are significant indicators of the diagnosis of infection and survival among decompensated cirrhotic patients. Combined CPR and NLR increase diagnostic ability of infection in the Child-Pugh C group. Decompensated cirrhotic patients with elevated CRP level and NLR should be carefully checked the presence of infection and considered for antibiotic therapy.

\section{PE-101 \\ Curative Treatment Provides a Better Survival Benefit in Elderly Patients with Hepatocellular Carcinoma}

June Sung Lee, Seung Dae Kang, Yun Ho Choi, Jong Wook Kim, Yoon Jung Jwa, Tae Jun Song, Won Ki Bae, Nam-Hoon Kim, Kyung-Ah Kim.

Department of Internal Medicine, Ilsan Paik Hospital, Inje University College of Medicine, Goyang, Korea

Background: Elderly patients with hepatocellular carcinoma (HCC) have much comorbidity, and curative treatment of HCC might increase treatment related mortality. The aim of this study was to compare the survival in elderly HCC patients treated with curative modalities including radiofrequency ablation (RFA), percutaneous ethanol injection (PEIT) and surgery to those treated with transcatheter arterial chemoembolization (TACE) and supportive care.

Methods: The medical records of patients with HCC older than 75 years who had visited a single tertiary medical center from January 2000 to December 2011 were reviewed. Clinical features, treatment modalities, and outcomes were investigated. Multivariable-adjusted hazard ratios (HR) for mortality with $95 \%$ confidence intervals (CI) were estimated using Cox proportional hazard models.

Results: Fifty-eight patients were analyzed. Twenty-nine patients ( $50 \%)$ were treated by TACE, 19 patients $(32.8 \%)$ by supportive care, and 10 patients $(17.2 \%)$ by curative treatment. Variables associated with survival were better Child-Pugh class, lower TNM stage. Treatment with curative intent showed significant survival benefit compared to TACE (HR for mortality, $0.10 ; 95 \% \mathrm{CI}, 0.01-0.95$ ). In a subgroup analysis of patients who had resectable HCC, TACE or curative treatment showed significant survival benefit over supportive care.

Conclusions: Curative treatment seems to have a better survival benefit in comparison with other treatment modalities even in elderly HCC patients.

\section{PE-102 \\ Case Report: Both Ischemic and Hemorrhagic Stroke after Liver Transplant Previous Diagnose of Protein C Deficit Patient}

Angelica Garcia Frans Serpa, Glenda Herrera,

Juan J. Suarez, Bernarda Jara, Gonzalo Dueñas,

Juan C. Aguilar, Juan J. Suarez,

Bernarda Jaram Gonzalo Duenas

Seoul National University, Seoul, Korea

Body: Neurological complications are consider the major source of morbidity and mortality related to liver transplant. The end stage liver disease (thrombocytopenia and abscence 
of coagulation factors) are consider a problem during and after liver transplant 2 . According to a review the Neurological complications are near the $20.1 \%$. The leading complications are Encephalopathy (11.8\%) and seizure (8.2\%), besides the incidence of immunosuppressive drug-related complications was about $1.7 \%$ for cerebral hemorrhage, and $1.2 \%$ for stroke. The $80 \%$ of the events were encountered in the first month after liver transplant, the majority of cases, seizure (50\%) presented in the first 2 weeks. 1

Case Report: Young female about 36 years old, with cronic autoinmune Hepatitis and Protein $\mathrm{C}$ deficit since five years ago. Cirrhosis outcome a year after. She went into a liver trasplant but two weeks after she had seizures and left paresia with inhability to speak or walk. Subsequently abnormalities were found in brain computed tomography (CT) and MRI, hemorrhagic and ischemic lesions. A month later dysarthria and paresia had marked recovery working with rehabilitation medicine service.

Radiographic Studies: CT reported a bruise located in the frontal left level with surrounding edema and effect of mass. MRI reported Bruise known about $6 \mathrm{~cm}$ of major diameter ( $6 \times 1.5 \times 1.2 \mathrm{~cm})$, multiple ischemic areas observed in addition at the cerebral stem and cerebellum.

Conclusions: Most complications occur early following liver transplant but may be seen even after 1 year and may survive serious neurologic events. 1 The cerebrovascular complications after liver transplant have severe impact on outcome of the patients. 2 In this case report the Protein $C$ deficit (coagulopathy) plus all factors of sick liver could trigger intracranial hemorrhage after liver Tx.

\section{Reference}

1 Bronster, D. J., et al. (2000), Central nervous system complications in liver transplant recipients - incidence, timing, and long-term follow-up. Clinical Transplantation, 14: 1-7. doi: 10.1034/j.1399-0012.2000.140101. 2. In hospital cerebrovascular complications following orthotopic liver transplantation: A retrospective study Li Ling ${ }^{1}$, et al., BMC Neurology 2008, 8:52 dci: 10.1186/1471-2377-8-52.

\section{PE-103}

\section{ICAM-1 shRNA Attenuates Liver Injury after Warm Ischemia Reperfusion}

Benjamin Navarro ${ }^{1,2}$, Xusheng Zhang ${ }^{2}$, Xiufen Zheng ${ }^{2}$, Anthony Jevnikar ${ }^{1,2}$, Weiping Min ${ }^{1,2}$, Douglas Quan ${ }^{1,2}$

${ }^{1}$ Multi-organ Transplant Program, London Health Sciences Center, ${ }^{2}$ Department of Surgery, Pathology, University of Western Ontario, Canada

Background: Liver ischemic reperfusion injury (LIRI) is linked to deleterious inflammatory effect associated with liver dysfunction and rejection in liver transplantation. LIRI results from oxygen and nutrient deprivation for a period of time followed by restoration of blood flow. ICAM-1 is a molecule that can mediate neutrophil involvement, which is associated with LIRI. One therapeutic approach to LIRI is inhibiting cell adhesion by blocking ICAM- 1 expression. In the present study we examine the effect of downregulating ICAM-1 gene expression using short hairpin RNA (shRNA) on protection liver from I/R injury.

Methods: shRNA vectors were constructed for ICAM-1 gene. Mice were treated with shRNA vectors by hydrodynamic tail injection 48 hours prior to LIRI. Induction of LIRI was conducted by interrupting blood supply to the left lateral and median lobes of the liver for 45 minutes followed by 6 hours of reperfusion. The effect of ICAM-1 shRNA was evaluated through examination of liver function by measurement of level serum alanine transferase (ALT) and aspartate transaminase (AST) as well as liver histopathology. Neutrophil accumulation was determined by myeloperoxidase (MPO) assay. Real time PCR was used to test gene silencing efficacy in vivo and in vitro.

Results: We demonstrated that LIRI stimulated an increase in ICAM-1 mRNA levels in liver tissue 6 hours after reperfusion. shRNA treatment effectively downregulated ICAM-1 gene expression in LIRI livers. ICAM-1 gene expression in the ICAM-1 shRNA treated group was 59\% lower than the scrambled shRNA group ( $\mathrm{P}<0.01)$. The serum level of ALT in the shRNA treated group was $62 \%$ lower than the scrambled $(\mathrm{P}<0.01)$ and AST in the shRNA treated group was 65\% lower than the scrambled shRNA group $(\mathrm{P}<0.05)$. In addition, neutrophil accumulation detected by MPO was significantly reduced in shRNA treated group $(\mathrm{P}<0.05)$. Furthermore, the ICAM-1 treatment group exhibited less tissue necrosis and tissue hemorrhaging than the scrambled group $(\mathrm{P}<0.05)$.

Conclusion: This study shows that the downregulation of ICAM-1 gene expression using shRNA attenuate LIRI via inhibition of neutrophil cell adhesion.

\section{PE-104 \\ Clinical Course of Consecutive 247 Hepatocellular Carcinoma Patients with Bile Duct Invasion at Initial Diagnosis}

\author{
Kwang-Sun Lee, Kang Mo Kim, Jihyun An, Danbi Lee, \\ Ju Hyun Shim, Young-Suk Lim, Han Chu Lee, \\ Young-Hwa Chung, Yung Sang Lee \\ Department of Gastroenterology, Asan Medical Center, \\ Seoul, Korea
}

Background: Bile duct invasion by hepatocellular carcinoma (HCC) is sometimes observed at the time of diagnosis or during treatment course of HCC. However, its prognostic implication and optimal treatment strategy has not been fully evaluated. The aim of this study was to investigate 
clinical course, treatment response and prognosis of HCC patients with bile duct invasion at the time of initial diagnosis.

Methods: Between January 2006 and December 2010, among 6,834 patients who visited Asan medical center with HCC for the first time, bile duct dilatation by HCC was observed in 247 patients. We respectively analyzed medical and radiological record of these patients.

Results: Among total 247 patients, median age was 56 years old and 206(83.4\%) were male. Child-Pugh class A, B and $\mathrm{C}$ was found in $115(46.6 \%), 105(42.5 \%)$ and $27(10.9 \%)$ patients. $88(35.6 \%)$ patients showed total bilirubin $\geq 3.0 \mathrm{mg} /$ $\mathrm{dL}$ and $51(58.0 \%)$ of them received percutaneous or endoscopic biliary drainage. Regarding American Joint Committee on Cancer (AJCC) stage, 55(22.3\%), 102(41.3\%) and $90(36.4 \%)$ patients was stage I-II, III and IV, and $15(6.1 \%)$, $8(3.2 \%), 197(79.8 \%)$ and $27(10.9 \%)$ was Barcelona Clinic Liver Cancer (BCLC) stage A, B, C and D. As an initial treatment, 105(42.5\%), 27(10.9\%), 9(3.6\%) and 6(2.4\%) patients received transarterial chemoembolization (TACE), surgical resection, systemic chemotheraphy and radiation therapy, respectively, and $100(40.5 \%)$ received only best supportive care (BSC). Among total 247 patients, median overall survival was 4.2 months and 1 year survival rate was $21.75 \%$. Among 197 patients with BCLC stage C, overall survival was significantly different according to the initial treatment type of HCC (surgical resection, TACE, other treatment versus BSC; median survival 12.7, 6.9, 2.7 and 1.8 months, $\mathrm{p}<0.001$ ). Multivariate analysis showed male, portal vein tumor thrombosis, advanced AJCC stage and HCC treatment were significant independent variables associated with overall survival.

Conclusions: Hepatocellular carcinoma with bile duct invasion at initial diagnosis had poor prognosis as a whole. However, even in BCLC stage C, aggressive local treatment such as surgery and TACE could improve overall survival of HCC patients with bile duct invasion.

\section{PE-105 \\ Coexistence of Hepatocellular Carcinoma and Intraperitoneal Follicular Lymphoma in Chronic Hepatitis C Patient}

Soon il Lee, Nae-Yun Heo, Seung Ha Park, Jong Ha Park, Tae Oh Kim, Young Soo Moon

Department of Internal Medicine, Haeundae Paik Hospital, Inje University College of Medicine, Busan, Korea

Background: Hepatitis C virus (HCV) is associated with not only hepatocellular carcinoma (HCC), but also with lymphoproliferative disorders such as non-Hodgkin's lymphoma (NHL). However, the case of coexistence of HCC and NHL is rarely reported.

Case: A 60-year-old man visited to the hospital complaining of abdominal discomfort. He had medical history of chronic HCV for 5 years, but had no antiviral therapy. Initial computed tomography (CT) scan showed $2 \mathrm{~cm}$ sized two hepatic nodules showing early enhancement and delayed wash-out. The magnetic resonance imaging (MRI) of liver presented another $0.8 \mathrm{~cm}$ sized small HCC. Initial CT showed multiple intrahepatic lymph node ( $\mathrm{LN}$ ) enlargements and only the largest $2 \mathrm{~cm}$ sized ellipsoidal LN in peri-portal area had high metabolic activity in positron emission tomographycomputed tomography (PET-CT). Clinical diagnosis was HCC with modified UICC stage of cT3NxM0. Explorative laparotomy for living donor liver transplantation was performed, and then excisional lymph node biopsy for peri-portal LN was reported as follicular lymphoma. Therefore, the operation was stopped, and the patient was referred to oncologist. Two cycles of R-CVP chemotherapy for follicular lymphoma with Ann Arbor stage I-A was done, and complete remission was accomplished. Then, two sessions of trans-arterial chemoembolization (TACE) and one of radiofrequency ablation (RFA) were performed to treat HCCs. The patient has survived for 2 years since the diagnosis of HCC and NHL. Another three small HCCs recurred, thus, further TACE was planned.

Conclusion: This case suggests that although intrahepatic LN enlargements are often considered as reactive change or metastatic lymphadenopathy in chronic hepatitis $\mathrm{C}$ patients with HCC, NHL also should be considered as differential diagnosis.

\section{PE-106 \\ The Computed Tomography Volumetry Estimates Graft Mass Accurately in Subjects with Fatty Liver \\ Yeonjung $\mathrm{Ha}^{1}$, Ju Hyun Shim ${ }^{2}$, Danbi Lee ${ }^{2}$, Kang Mo Kim², Young-Suk Lim², Han Chu Lee, Young-Hwa Chung ${ }^{2}$, Yung Sang Lee ${ }^{2}$, Eunsil Yu ${ }^{3}$, Sung-Gyu Lee 4 \\ ${ }^{1}$ Department of Internal Medicine, ${ }^{2}$ Department of Gastroenterology, Asan Liver Center, ${ }^{3}$ Department of Pathology, ${ }^{4}$ Division of Hepatobiliary Surgery and Liver Transplantation, Department of Surgery, Asan Medical Center, University of Ulsan College of Medicine, Seoul, Korea}

Background: The volume measurement is important in successful liver transplantation and hepatic resection. The computed tomography volumetry, the imaging-based volumetric method, is known to be an accurate tool in estimating actual graft mass. The aim of this study is to demonstrate whether computed tomography volumetry also estimates graft mass accurately in fatty livers, where the volume is greater than the mass.

Methods: The healthy liver donors were divided into 4 groups according to the degree of fatty changes on preoperative biopsy; < 5\% (group 1), 5-9\% (group 2), 10-19\% (group 
3 ), and $\geq 20 \%$ (group 4). The graft mass estimation error (\%) was calculated as follows (measured volume - graft mass)/ (graft mass) x 100. The difference of graft mass estimation error (\%) across the groups was examined by Kruskal-Wallis test. A $p$-value of $<0.008$ was considered statistically significant according to the Bonferroni adjustment. The agreement between measured volume and graft mass across the groups was evaluated with intraclass correlation coefficient.

Results: 752 subjects $(72.2 \%$ male, $28.4 \pm 8.6$ years) were enrolled. Baseline parameters were comparable except for increasing body mass index, alanine aminotransferase level, and gamma glutamyl transpeptidase level as the group number increases (from group 1 to 4 ), as expected. The difference of graft mass estimation error (\%) among 4 groups did not show statistical significance $(p=0.124) ; 1.5 \pm 13.8 \%$, $2.8 \pm 14.4,3.8 \pm 14.7$, and $6.0 \pm 16.3$ in the group 1 through 4 . There was almost perfect agreement between measured volume and graft mass, with the intraclass correlation coefficient of 0.94 in group 1 and 2, 0.93 in group 3, 0.97 in group 4, and 0.94 overall $(p<0.001)$.

Conclusions: Our data indicates that regardless of the degree of fatty changes, computed tomography volumetry can be used as an accurate tool for estimating liver volume in candidates of liver donation or hepatectomy.

\section{PE-107 \\ Identification of EGR1 as a Key New Mediator of the Differentiation of BM-Derived Mesenchymal Stem Cells into Functional Hepatocytes with Mesenchymal Epithelial Transition \\ Hye-Lim Kim Jung Hoon Cha, Na Ri Park, Si Hyun Bae, Jong Young Choi, Seung Kew Yoon \\ College of Medicine, The Catholic University of Korea, Seoul, Korea}

Background: MET and the reverse process, epithelial-tomesenchymal transition (EMT), both occur in normal tissue, including gastrulating and regenerating tissue. MET in hepatic stem cells is important to multiple processes, including hepatogenic differentiation and liver development, regeneration.

Early growth response (EGR) 1, transcription factor of early growth response gene family, is induced in the responses to a number of growth and differentiation factors. The aim of this study is to investigate that EGR1 acts as a key regulator of EMT/MET in the process of hepatogenic differentiation from bone marrow-derived mesenchymal stem cells (BM-MSCs).

Methods: Based on the 2 step protocol, BM-MSCs were cultured for hepatogenic differentiation. Pas staining and urea production test were performed to verify the functionality of hepatogenic differentiation. To search for novel proteins in BM-MSCs before and after differentiation, Protein/DNA array was performed. To determine the effect of EGR1, BM-MSCs were infected by lenti-shEGR1. EMT markers (N-cadherin, vimentin, a-SMA) and MET markers (E-cadherin, CK18) were identified during the hepatogenic differentiation of infected BM-MSCs.

Results: During differentiation proceeds, from day 1 to day 28 , stem cells change in shape from spindle to polygon with larger size and tighter intercellular connections. Intracellular glycogen and urea production was significantly increased from day 1 to day 28. The mRNA expression of liver-specific genes was upregulated. In order to study key regulator in hepatogenic differentiation, we performed Protein/DNA array. EGR1 in differentiated BM-MSCs were significantly increased during the culture compared to undifferentiated BM-MSCs. Therefore, BM-MSCs were differentiated after infected by lenti-shEGR1. The downregulation of the EGR1 expression decreased MET related genes and hepatogenic differentiation.

Conclusions: In this study, we identified novel factors in the process of hepatogenic differentiation through inducing the MET process. Our study suggests that EGR1 were expected to the "transcription factor therapy" as novel strategy in future clinical treatment.

\section{PE-108 \\ Chronic Liver Diseases in Mongolia}

O. Baatarkhuu, N. Tuvshinjargal, T. Alimaa, J. Amarsanaa, R. Otgonbayar

Health Sciences University of Mongolia, Mongolia

Background: Mongolia is known for its high endemicity for HBV, HCV, and HDV infections among apparently healthy populations. However there are little or no data on the prevalence and genotype distribution of HBV, HCV and HDV among patients with chronic liver diseases in Mongolia.

Methods: Serum samples obtained in 2009 from 207 patients (51.0 \pm 11.9 years) including those chronic hepatitis $(n=90)$, liver cirrhosis $(n=41)$, and HCC $(n=76)$ were tested for serological and molecular markers of HBV, HCV, and HDV infections.

Results: Of the 207 patients, 144 (69.6\%), 106 (51.2\%), and 117 (56.5\%) tested positive for HBsAg and HBV DNA, HCV RNA, and HDV RNA, respectively.

Collectively, 172 patients $(83.1 \%)$ were viremic for one or more of these viruses, including dual viremia of $\mathrm{HBV} / \mathrm{HDV}$ (26.6\%) or $\mathrm{HBV} / \mathrm{HCV}$ (7.7\%) and triple $\mathrm{HBV} / \mathrm{HCV} / \mathrm{HDV}$ viremia (30.0\%). Of note, triple ongoing infection was significantly more frequent among patients with HCC than among those with chronic hepatitis (63.2\%) vs. $14.4 \%$, p $\leq 0.0001$ ). The distribution of HBV genotypes among the 116 HBVviremic patients was: A (0.9\%), B (0.9\%), C (6.0\%), 
D (88.8\%), and C plus D (3.4\%). All 117 HDV isolates were clasisifired into genotype 1 . The $106 \mathrm{HCV}$ RNA positive samples were typed as genotype $1 \mathrm{~b}(92.5 \%)$.

Conclusions: The present study revealed that ongoing dual or triple infection of HBV, HCV and HDV is highly prevalent among patients with chronic liver diseases of Mongolia.

\section{PE-109 \\ The Effects of Application of Autologous Hematopoietic Stem Cells (AHSCs) in Patients with Primary Biliary Cirrhosis (PBC) Resistant to Drug Treatment}

Ilyassova B.S., Kayupov B.A., Shaymardanova G.M., Askarov M.B.

National Scientific Research Medical Center, Astana, Kazakhstan

Background: $\mathrm{PBC}$ is further characterized by highly specific serum antimitochondrial autoantibodies (AMA) and autoreactive $\mathrm{T}$ cells, a striking female predominance, a strong genetic susceptibility. It is proposed, the AHSCs influence on the molecules of autoreactivity and then the proinflammatory cells are eliminated. Consequently generation new naïve T-lymphocytes in patients is interrupted, it results to the restoration of the tolerance to autoantigens.

The alm of the study: to estimate efficiency of application of AHSCs in the primary biliary cirrhosis.

Methods: 10 patients with primary biliary cirrhosis: 4 with the II morphological stage PBC, 2 - III stage, 2- IV stage and 2 - with overlap syndrome: PBC IIIst+AIH resistant to the standart therapy (UDCA and methotrexate) have been included to the study. The AHSCs has been realized by 2 stages: 1-aspiration of bone marrow suspension in the amount $200 \mathrm{ml}$; phenotyping of cells by cellcytometer BD; isolation of the mononuclear fraction and precultivation during 24-48 hours. 2 - intravenous transplantation of the cells suspension. The cytokines levels have been investigated in the cell supernatant and in the blood serum by ELISA.

Results: 7 days after transplantation of AHSCs the tests have shown the decrease of ALT level from 1,930 $\pm 0,300$, Std. deviation (SD) 0,949 , to $1,466 \pm 0,260, S D=0,821, p=0,007$, of AST level from $1,926 \pm 0,415, \mathrm{SD}=1,313$ to $1,384 \pm 0,207$, $\mathrm{SD}=0,655, \mathrm{p}=0,005$. The total bilirubin level decreased from $60,184 \pm 22,539 \mathrm{mkmol} / \mathrm{l}, \mathrm{SD}=71,275$ to $43,619 \pm 17,498$, $\mathrm{SD}=55,334, \mathrm{p}=0,005$, the level of direct bilirubin decreased from $44,751 \pm 20,605, \mathrm{SD}=65,158$, to $20,074 \pm 11,447$, SD $=$ $36,200, \mathrm{p}=0,007$. Serum protein $69,688 \pm 2,457, \mathrm{SD}=7,770$, to $77,465 \pm 1,172, \mathrm{SD}=3,706, \mathrm{p}=0,005$ and serum albumin $39,712 \pm 2,853, \mathrm{SD}=9,0197$, to $44,088 \pm 2,619, \mathrm{SD}=8,282, \mathrm{p}=$ 0.028 increased. Three months later the levels of IL-10, IL-12p70, IL-17, IL-2 and IL-4 did't change significantly, IFN- $\gamma$ level was increased $(p=0,005)$. IL-1 $\beta$ level $(p=0.006)$ and TNF- $\alpha$ level $(p=0,005)$ decreased. The SF36 results showed significant improvement. The morphological study was able to spend in 8 patients with the II, III stage PBC and with overalap syndrome. The results of morphological exams showed the decrease of the portal tructs infiltration by lymphocytes, plasmocytes, decrease of the dystrophia of hepatocytes.

Conclusion: The application of AHSCs by immunological effect affects on the histological activity of liver. Possibly it is promising method of treatment of primary biliary cirrhosis

\section{PE-110 \\ FK506 Accelerates $\mathrm{CoCl} 2$ - induced Cytotoxicity via ER Stress Pathway}

Soo Jin Na Choi, Sang Young Chung

Department of Surgery, Chonnam National University Hospital, Gwangju, Korea

Background: The effects of FK506 on the endoplasmic reticulum (ER) mediated stress pathway accelerates $\mathrm{CoCl}_{2}-$ induced cytotoxicity in human hepatoma HepG2 cell line were investigated.

Methods: We examined the effects of FK506 on $\mathrm{CoCl}_{2}-$ induced cytotoxicity by western blottings of poly ADP-ribose polymerase (PARP), CHOP, GRP78, Nrf2, ATF4, ATF6, XBP-1, $\mathrm{Bak}, \mathrm{Bax}$, and $\mathrm{Bcl}-2$. And the catalytic acitivity of caspase- 3 and -12 caspase in HepG2 cells was also measured.

Results: $\mathrm{FK} 506$ and $\mathrm{CoCl}_{2}$ significantly induces the synergistic effect of HepG2 cytotoxicity in dose dependent manner. Increased active-PARP expression occurred at 24 hours after FK506 treatment on cobalt chloride-induced HepG2 cytotoxicity and peak activation of cleaved caspasec-3 was also observed at 24 hours. FK506 aggravates cobalt chlorideinduced HepG2 cytotoxicity. GRP78 expression was increased 24 hours after FK506 treatment on cobalt chloride-induced HepG2 cytotoxicity. CHOP and caspase-12 expressions were increased 24 hours after FK506 treatment on cobalt chlorideinduced HepG2 cytotoxicity. Expressions of ATF4 and ATF6 were same manners. Expression of XBP-1 was decreased beginning at 6 hours. FK506 exasperate endoplasmic reticulum stress by cobalt chloride-induced cytotoxicity. Bcl-2 protein expression decreased, but FK506 induces expression of Bak and Bax by cobalt chloride-induced cytotoxicity. Nrf2 expression was also noted.

Conclusions: FK506 and $\mathrm{CoCl}_{2}$ significantly induces the synergistic effect of cytotoxicity in dose dependent manner. FK506 aggravates cobalt chloride-induced cytotoxicity. FK506 exasperate endoplasmic reticulum stress by cobalt chlorideinduced cytotoxicity. FK506 accelerates expression of ER-stress related nuclear transcriptional factor. 